Genetic constraints that determine rhizobium-root nodule formation in Parasponia andersonii 


\section{Thesis committee}

\section{Promotor}

Prof. Dr T. Bisseling,

Professor of Molecular Biology

Wageningen University

\section{Co-promotor}

Dr R. Geurts

Associated professor, Laboratory of Molecular Biology

Wageningen University

\section{Other members}

Prof. Dr M.E. Schranz, Wageningen University

Dr R.H.M. Op den Camp, Keygene N.V.,Wageningen

Dr H.W.M. Hilhorst, Wageningen University

Dr H.J. Schouten, Wageningen University

This research was conducted under the auspices of the Graduate School of Experimental Plant Science (EPS). 


\title{
Genetic constraints that determine rhizobium-root nodule formation in Parasponia andersonii
}

\author{
Maryam Seifi Kalhor
}

Thesis

submitted in fulfilment of the requirements for the degree of doctor

at Wageningen University

by the authority of the Rector Magnificus

Prof. Dr A.P.J. Mol,

in the presence of the

Thesis Committee appointed by the Academic Board

to be defended in public

on Wednesday 5 October 2016

at 4.00 p.m. in the Aula. 
Maryam Seifi Kalhor

Genetic constraints that determine rhizobium-root nodule formation in Parasponia andersonii

160 pages

$\mathrm{PhD}$ thesis, Wageningen University, Wageningen, NL (2016)

With references, with summary in English

ISBN: 978-94-6257-911-8

DOI: $10.18174 / 388906$ 


\section{CONTENTS}

Chapter 1 General introduction 1

Chapter 2 Efficiency of Agrobacterium rhizogenes-Mediated Root 27 Transformation of Parasponia and Trema Is Temperature Dependent

Chapter 3 Agrobacterium tumefaciens-mediated Stable Transformation of Trema 43 tomentosa

Chapter 4 Exogenous Nitrate Interferes with the Switch from Infection to 61 Fixation Thread Formation in Parasponia andersonii Root Nodules

Chapter 5 Symbiotic Functioning of the GRAS-TYPE Transcriptional Regulators NSP1 and NSP2 is Conserved in the Non-legume Parasponia andersonii

Chapter 6 Dual Effect of Ethylene on Root Nodulation of Parasponia andersonii 105

Chapter 7 General discussion

Summary 
TO MY DEAR HUSBAND

SASAN 


\section{Chapter1}

General Introduction 


\section{Chapter 1}

\section{Biological nitrogen fixation}

Nitrogen $(\mathrm{N})$ is the seventh most abundant element on earth, and in living cells it is required in large amounts as it is a component of proteins, nucleic acids and other cellular constituents. About $80 \%$ of the earth atmosphere is made up of di-molecular nitrogen $\left(\mathrm{N}_{2}\right)$ gas (Lum and Hirsch, 2002). However, $\mathrm{N}_{2}$ gas cannot be used by most organisms, including plants (Vance, 2001). Plants can only utilize reduced forms of nitrogen like ammonium $\left(\mathrm{NH}_{4}{ }^{+}\right)$and nitrate $\left(\mathrm{NO}_{3}{ }^{-}\right)$(Hirel et al., 2007). They acquire these forms of fixed nitrogen for example by; 1) application of chemical fertilizer, 2) the release of these compounds during decomposition of organic matter, 3) atmospheric nitrogen that is converted into ammonia and nitrate by lightning and entering soil by rainfall and/or 4) biological nitrogen fixation.

Biological nitrogen fixation can be conducted by a limited number of prokaryotes. Some of these bacteria can conduct nitrogen fixation in a free-living state, whereas others can do so only in association with plants. As biological nitrogen fixation is an energy demanding biochemical reaction, highest efficiencies of nitrogen fixation are obtained when the bacteria can retrieve carbon from a host plant. In return, the microbe can deliver ammonia, providing a selective advantage to the plant under nitrogen limiting conditions. Ultimately, such interactions can evolve in a stable symbiosis, giving profit to both partners. Among the best studied nitrogenfixing symbiosis is the interaction between legumes and rhizobia.

Symbiotic nitrogen-fixing rhizobia represent 15 genera; 13 within the $\alpha$-proteobacteria (among which Rhizobium, Sinorhizobium, Mesorhizobium and Bradyrhizobium are most prominent) and 2 genera within $\beta$-proteobacteria (Burkholderia and Cupriavidus, respectively). All these bacteria combine two genetic traits; namely a set of nitrogen fixation (nif) genes that encode the nitrogenase enzyme complex, and a set of nodulation (nod) genes that allow them to synthesize lipo-chitooligosaccharide (LCO) molecules (also known as Nod factors). These LCOs are in structure very similar to LCOs that are produced by Arbuscular Mycorrhizal (AM) fungi of the order Glomerales (Gough and Cullimore, 2011). Secreted LCOs can act as signaling molecules to establish infection of plant roots. AM fungi are obligate biotrophs that need to form feeding structures -known as arbuscules - in root cortical cells. In these cells, the fungus supplies minerals, especially phosphates, to the plant to retrieves carbohydrates. The AM fungus-plant symbiosis is widespread. It is estimated that more than $80 \%$ of today's plant species can establish an AM symbiosis (Parniske, 2008). Detection of AM fungi in fossilized plant records indicates 
that the association with AM fungi evolved more than 400 million years ago, and possibly was important for the evolution of land plants in mid-Paleozoic era (Read et al., 2000). By contrast, the $\mathrm{N}_{2}$-fixing rhizobium symbiosis in legumes is much younger, presumably originating 60 million years ago (Sprent, 2008). Production of LCOs by symbiotic rhizobia suggests that these can activate the LCO signaling pathway of many plant species. However, $\mathrm{N}_{2}$-fixing rhizobium symbiosis evolved only in two lineages; the legume family (Fabaceae) and the genus Parasponia in the Cannabis family (Cannabaceae).

Rhizobia require specialized organs -known as nodules- to commit nitrogen fixation. Nodules are generally formed on the root of the plant. Legume nodules are highly differentiated organs that consist of a large central zone surrounded with several vascular bundles to facilitate transport of nutrients. The central zone of the nodule is surrounded by a layer of suberized cells that form an oxygen barrier (Nap and Bisseling, 1990). Cells of the central zone can contain hundreds of rhizobia that are differentiated in nitrogen fixing organelle-like structures. These structures are named symbiosomes and produce ammonia (Roth and Stacey, 1989). Legumes evolved several mechanisms to further optimize the symbiotic interaction, some of which are lineage specific. For example, many legumes evolved a mechanism to select specific rhizobial species or even strains (Martínez-Romero, 2009). Additional mechanisms control nodule number or terminate the symbiosis when sufficient exogenous nitrates can be retrieved from soil, or when rhizobia remain in default (Streeter and Wong, 1988). In contrast to legumes, Parasponia root nodules are less sophisticated. Parasponia nodules have a single -central- vascular bundle and bacteria remain in thread-like structures, known as fixation threads (Behm et al., 2014). Additionally, Parasponia is highly promiscuous for rhizobium, and even makes nodules with strains that default in nitrogen fixation (Op den Camp et al., 2012).

\section{LCO signaling pathway}

By exploiting Lotus japonicus (Lotus) and Medicago truncatula (Medicago) as research models, insight has been obtained in the genetic networks underlying the formation of root nodules. Especially, the LCO induced genetic network controlling nodule formation and bacterial invasion has been uncovered (Geurts et al., 2005; Geurts et al., 2016). Rhizobium LCOs have a basic structure consisting of a backbone of three to five $\mathrm{N}$-acetyl-glucosamine residues that are acylated at the non-reducing amino group with a fatty acid of 16-20 C-atoms in length (C16 to 


\section{Chapter 1}

C20). Additional substitutions to the backbone and/or unsaturated bounds in the acyl chain can be present and play a role in the host specificity of the symbiosis (D'Haeze and Holsters, 2002). In legumes, rhizobium LCOs are recognized by a heterodimeric complex of two types of LysM (Lysin motif) receptor kinases (LysM-RLK), which activate a signaling pathway that results in activation of a transcriptional network (Broghammer et al., 2012). These receptors are named LjNFR1 and LjNFR5 in Lotus and MtLYK3 and MtNFP in Medicago. LysM domains are predicted to be sites of interaction with $\mathrm{N}$-acetyl-glucosamines-containing compounds (Steen et al., 2005). In case of LjNFR1/MtLYK3 and LjNFR5/MtNFP it is found that these receptors bind LCOs. LjNFR1 and LjNFR5 receptor proteins directly bind to the LCOs with high affinity (Broghammer et al., 2012). All LysM-RLKs contain three diverged LysM domains. In Medicago two main LysM-RLK classes are recognized, named LysM-I (containing $L Y K$ genes, including MtLYK3) and LysM-II (containing LYR genes as well as MtNFP). Genes of both classes are distinct in their intron/exon organization, and whether a canonical kinase domain is present. $L Y K$ genes contain 10 to 12 exons, and encode proteins with functional kinase domains. In contrast, LYR genes are generally single exon genes, and contain a kinase domain that lacks an activation loop. Such kinases are considered to be inactive. LYR proteins therefore most probably will function in a heterodimeric complex. Studies with of LjNFR1/LjNFR5 and MtLYK3/MtNFP indicated that such complexes are indeed formed, which suggests that LCO signaling is committed by such heterodimeric complex (Madsen et al., 2003; Madsen et al., 2011; Arrighi et al., 2006; Pietraszewska-Bogiel et al., 2013) Besides MtLYK3 and MtNFP, the LysM-RLK MtLYR3 of Medicago has been identified as a high affinity LCO binding protein (Malkov et al., 2016). This suggests that possibly different type of receptor complexes can be formed to fine tune symbiotic LCO signaling.

Besides $L j N F R 1 / M t L Y K 3$ and $L j N F R 5 / M t N F P$, a series of other genes have been identified to be essential for LCO signaling including: LjSYMRK/MtDMI2, LjCASTOR, LjPOLLUX/MtDMI1, LjNUP133, LjNUP85, LjNENA, CCAMK and the transcriptional regulators $L j C Y C L O P / M t I P D 3$, NIN, MtERN, NSP1 and NSP2. Knockout mutations in any of these genes led to major defects in, or even complete loss of, nodule initiation (Kistner et al., 2005; Oldroyd et al., 2009; Parniske, 2008).

LjSYMRK/MtDMI2 encodes a trans-membrane receptor-like kinase protein with three Leucinerich repeat (LRR) domains in the predicted extracellular region. It is located in the plasma 
membrane and studies in Lotus show that it interacts with LjNFR1 and LjNFR5 (Chen et al., 2012). Mutation in the LjSYMRK/MtDMI2 gene abolishes rhizobium LCO signaling. Activation of the LCO receptors in conjunction with $L j S Y M R K / M t D M I 2$ induces intracellular signaling, which is associated with oscillations of the calcium concentration in the perinuclear region. For this a perinuclear cation channel (LjCASTOR and $\mathrm{Lj} P O L L U X / M t D M I 1)$ and 3 components of the nuclear pore including; Lotus NUCLEOPORIN 85 (LjNUP85), LjNUP133 and LjNENA are essential (Groth et al., 2010; Saito et al., 2007; Kanamori et al., 2006). The LCO induced calcium oscillation signal is -most probably- decoded by a nuclear localized calcium and calmodulin dependent kinase CCaMK (also named MtDMI3 in Medicago). Activation of CCaMK triggers a transcriptional network, starting with the interacting protein LjCYCLOPS/MtIPD3, yet the only known target of CCaMK. By phosphorylation, LjCYCLOPS is released from the complex with CCaMK, and become an active transcription factor. LjCYCLOPS binds to the promoter of the ERNI and NIN gene and activates its transcription in a phosphorylation-dependent manner (Singh et al., 2014; Kistner and Parniske, 2002). Mutations in these genes interfere with LCO signaling, resulting in a nodulation phenotype. Interestingly, all genes from $L j S Y M R K / M t D M I 2$ down to the activation of LjCYCLOPS/MtIPD3 are also important for AM-symbiosis.

NIN (NODULE INCEPTION) is a nodulation-specific gene that encodes a transcription factor and acts downstream of the LCO signaling pathway. Nodule organogenesis and infection are fully blocked in nin knockout mutants (Marsh et al., 2007; Schauser et al., 1999). Downstream targets of NIN include genes encoding subunits of the Nuclear Factor Y (NF-Y) CCAAT-boxbinding heterotrimeric transcription factor complex. The NF-Y complex consists of the three distinct proteins called NF-YA, NF-YB and NF-YC, all encoded by a small gene family (Kahle et al., 2005). In legumes several members of these proteins have been found to commit a function in rhizobium symbiosis. In Lotus, LjNF-YAI and $L j N F-Y B 1$ are transcriptional targets of LjNIN and promoting cortical cell divisions (Soyano et al., 2013). Knock down studies of MtNF-YA1 in Medicago revealed that a symbiotic NF-Y transcription complex commits functions that are essential in nodule development. MtNF-YA1 RNAi nodules vary in size, but are always smaller than wild type nodules. Small MtNF-YAl knock down nodules lack a meristem, instead contain only fully infected cells (Xiao et al., 2014). This suggests that MtNFYA1 is required for formation and maintenance of the nodule meristem in Medicago. 


\section{Chapter 1}

$E R N$ belongs to the APETELLA2 / Ethylene Response Factor (AP2/ERF) family of transcription factors. MtERN1 Studies in Medicago showed that MtERN1 in conjunction with two GRASType transcriptional regulators, MtNSP1(Nodulation Signaling Pathway1) and MtNSP2, is required for rhizobium LCO induced expression of the early nodulin gene, MtENOD11, in the root epidermis (Cerri et al., 2012). Knockout mutations in MtNSP1 or MtNSP2 are unable in functional LCO signaling, whereas this is not the case for Mtern1 knockout plants. A knockout mutation in MtERN1 hampers nodulation, but mutant plants show early symbiotic responses including rhizobial infection. This suggest the existence of overlapping expression patterns with different gene namely MtERN2, a close homolog of MtERN1. Genetic analysis on these genes indicate that MtERN1Mt/ERN2 coordinately induce rhizobial infection and nodule organogenesis (Cerri et al., 2016).

MtNSP1 has been shown to bind to the promoter of MtENOD11, which requires MtNSP2 (Hirsch et al., 2009). Yeast-two hybrid studies as well as split YFP studies indicate that MtNSP1 and MtNSP2 function in homo- and heteromeric complexes (Heckmann et al., 2006). It is also reported that MtNSPl is involve in arbuscular mycorrhizal symbiosis, though not absolutely essential (Delaux et al., 2013). Furthermore, a recent study showed that nsp2 mutants in Medicago do not respond to Myc- LCOs and are colonized less than wild-type plants by the AM fungus Rhizophagus irregularis (Maillet et al., 2011). In addition, MtNSP1 and MtNSP2 also have been shown to be involved in strigolactone biosynthesis. In this study a Mtnsp1Mtnsp2 double mutant showed reduced colonization by AM fungi, which probably is caused by reduced strigolactone secretion, a compound that triggers branching of fungal hyphae (Liu et al., 2011).

\section{The rhizobium and AM fungal symbiosis share signaling and cellular processes}

Genetic studies in Pea and later, in Medicago and Lotus have revealed that a major part of the rhizobium LCO signaling pathway is shared with the signaling pathway that is activated by AM fungi Therefore, this genetic signaling network is named the common symbiosis signaling pathway, or symbiotic toolkit (Parniske, 2008). Components of the common signaling pathway are SYMRK (Endre et al., 2002; Stracke et al., 2002), Castor and Pollux (Ané et al., 2004; Imaizumi-Anraku et al., 2005) CCaMK (Lévy et al., 2004; Mitra et al., 2004; Tirichine et al., 2006) and Cyclops (Messinese et al., 2007; Chen et al., 2008; Yano et al., 2008), NSP1 (Takeda et al., 2013) and NSP2 (Maillet et al., 2011). Mutations in these genes affect both endosymbiotic 
interactions. In legumes, no LCO receptors have been found to commit a function in rhizobium as well as endomycorrhizal symbiosis. However, like rhizobia, AM fungi produce LCOs. These are named Myc factors and are structurally similar to Nod factors (Gough and Cullimore, 2011). This suggests that the receptors for Myc factors will be similar to those for Nod factors.

Parasponia plants are the only non-legumes that can also establish a nodule symbiosis with rhizobium. Knock down of the Parasponia LCO receptor gene, PaNFP, results in a block of intracellular infection nodules (den Camp et al., 2011). Knock down of this receptor also blocks arbuscule formation by AM-fungi, whereas the roots are still intercellularly colonized (den Camp et al., 2011). Parasponia has only a single NFP (LysM-type like) gene, whereas in legumes this gene experienced a duplication event. As will be described in more detail below, the Parasponiarhizobium symbiosis evolved relatively recent in comparison to the legume-rhizobium symbiosis. Therefore, it seems probable that in Parasponia, the PaNFP LCO receptor didn't neofunctionalized to function exclusively in rhizobium symbiosis, but this receptor still has its ancestral function, which is perception of Myc factors. Such role of NFP-like genes in mycorrhizal symbiosis is supported by studies in tomato on SILYK10. SILYK10 is the ortholog of PaNFP. In tomato, absence of mycorrhization was observed in knock down mutants of SlLYK10 (Buendia et al., 2015). This suggests that a controlling arbuscule formation in AM symbiosis is the ancestral function of $P a N F P / S I L Y K 10$ genes.

Activation of the common symbiosis signaling pathway -either by rhizobium or AM-fungiinduces cellular processes that lead to intracellular accommodation of the microbe. In both symbioses this involves the formation of a host membrane structure by which the microbe is guiding into the cells (Gutjahr and Parniske, 2013; Ivanov et al., 2012). Anytime the microbe remains bound by plant derived membrane structures; symbiosomes or arbuscules (Limpens et al., 2009), These symbiotic membrane compartments facilitate nutrient exchange between both partners (Geurts and Vleeshouwers, 2012). Studies in Medicago have revealed that specific vesicle-SNAREs (soluble N-ethylmaleimide sensitive factor attachment protein receptor) belonging to the VAMP72 (vesicle-associated membrane protein) family are involved in symbiosome as well as arbuscule formation. VAMP72 proteins are active in exocytotic pathways and mark secretory vesicles that accumulated in the cellular entry point of the microbe (Ivanov et al., 2012). This shows that despite of morphological differences, rhizobium and mycorrhizal 


\section{Chapter 1}

fungi trigger similar cellular responses. So, in addition to the LCO signaling pathway, also the pathway controlling symbiosome formation has been co-opted from the AM fungal symbiosis. Root nodule symbiosis is not limited to nitrogen fixing rhizobia. Also some gram positive filamentous Frankia bacteria can establish a nodular nitrogen fixing symbiosis on a selective group of $\sim 220$ plant species. These so-called actinorhizal plants belong to eight families of the Fabid clade, suggesting multiple evolutionary origins of this symbiosis (Diédhiou et al., 2014; Doyle, 2011). Although it remains unclear whether Frankia species produce LCOs or use an alternative signal to communicate with their host plants, reverse genetic studies indicated that -at least part of- the common symbiosis signaling pathway is used to establish the Frankiaactinorhizal plant symbiosis. Studies in the actinorhizal plants Casuarina glauca and Datisca glomerata revealed the symbiotic functioning of $C g S Y M R K / D g S Y M R K, C g C C a M K$ and $C g N I N$. (Gherbi et al., 2008; Markmann et al., 2008; Clavijo et al., 2015). Furthermore, Frankia induced signaling triggers a $\mathrm{Ca}^{2+}$ oscillation response in C. glauca and Alnus glutinosa (Granqvist et al., 2015; Chabaud et al., 2016). These findings strongly suggest that evolution of a nitrogen-fixing endosymbiosis with either rhizobium or Frankia was guided by genetic constraints.

Assuming that AM fungi infect their various hosts by a conserved mechanism, it implies that the genes encoding components for LCO signaling as well as the symbiosis related exocytosis pathway will be widespread in the plant kingdom. This is well supported by the genome sequence analysis of several plant species. Species that are able to establish an AM symbiosis harbor putative orthologues of the symbiosis genes identified in legumes, whereas often these genes are absent in AM non-hosts (Delaux et al., 2014; Bravo et al., 2016). For example in Arabidopsis, which is non-host for AM fungi, most genes of the common signaling pathway as well as MtNFP/LjNFR5 have been lost. This further supports the idea that genetic constraints determined the evolution of the rhizobium endosymbiosis. However it arise the question why not more plant species have gained the rhizobium symbiosis trait? Taken into account the recent research on Parasponia, comparative studies of Parasponia and legumes as well as Parasponia and its non-nodulating sister species Trema can answer this question, and may pave the way for future transfer of rhizobium symbiosis to the other non-legume plants.

\section{Effect of fixed nitrogen sources on legume root nodule formation}

Legumes only form root nodules under nitrogen limiting condition, whereas the addition of 
excessive amounts of combined nitrogen suppress nodulation (Barbulova et al., 2007). Also, nitrogen fixation can cease in existing nodules by addition of an exogenous fixed nitrogen source. Legumes control the total number of nodules formed, because the maintenance of nitrogen fixing rhizobia is energy demanding (Reid et al., 2011; Schnabel et al., 2011). Competence of leguminous plants to form nodules is determined by physiological conditions. The mechanisms by which fixed nitrogen suppress the different steps of root nodule formation is only partially understood (Barbulova et al., 2007).

The best studied mechanism to control nodule numbers is autoregulation of nodulation (AON). AON is a negative feedback regulation by which the number of nodules and the nodulation zone are tightly restricted. Mutants in AON have lost the ability to control nodule number and therefore form numerous nodules (so-called super- or hypernodulators). AON mutants have been studied in several legumes like Pea, Soybean, but also the model legumes Medicago and Lotus. This has resulted in the identification of a CLAVATA1-like LRR receptor kinase (LRR-RLK) named HYPERNODULATION ABERRANT ROOT FORMATIONI (LjHARl) in Lotus (Kawaguchi et al., 2002), NODULE AUTOREGULATION RECEPTOR KINASE (GmNARK) in Soybean (Olsson et al., 1989) and SUPER NUMERIC NODULES (MtSUNN) in Medicago (Elise et al., 2005). LjHAR1, GmNARK and MtSUNN are most probable orthologous genes that upon mutation results in a supernodulating phenotype. Ljhar1, Gmnark and Mtsunn knockout mutants also have lost the ability to suppress nodulation in presence of exogenous fixed nitrogen (Barbulova et al., 2007; Carroll et al., 1985; Magori et al., 2009). Grafting experiments showed that LjHAR1, GmNARK and MtSUNN are only essential in the shoot and are key factors in systemic root-to-shoot-to-root negative feedback regulation of nodulation. The encoded LRRRLK recognizes CLV3/embryo-surrounding region (CLE) peptides (Hirakawa et al., 2008). CLEs are a group of small (12-13 amino acids) secreted peptides that are derived from the Cterminal region of pre-proteins. 39 LjCLE genes have been identified in the Lotus genome, three of them, LjCLE-RS1, LjCLE-RS2 and LjCLE3, are significantly up-regulated in Lotus nodulated roots. Transcription of $L j C L E-R S 1$ and $L j C L E-R S 2$ is responsive to exogenous nitrate and rhizobial inoculation (Okamoto et al., 2009). When LjCLE-RS1 or LjCLE-RS2 is ectopically expressed in roots of the Ljharl mutant using an Agrobacterium rhizogenes-mediated transformation system, both transformed and non-transformed roots show a super-nodulation phenotype. This indicates that these two genes have suppressive effect on nodulation in a 


\section{Chapter 1}

LjHAR1 dependent manner (Okamoto and Kawaguchi, 2015). LjCLE-RS2 was found to be strongly up-regulated in response to nitrate. Therefore it is hypothesized that LjCLE-RS2 translocates from root-to-shoot and directly binds to the LjHAR1 receptor (Okamoto et al., 2013). Based on these findings, a model for the long-distance nitrate inhibition of nodulation has been proposed in Lotus. In this model, the LjCLE-RS2/LjHAR1 long-distance signaling system is shared between nitrate inhibition and AON (Okamoto and Kawaguchi, 2015).

A different study in Medicago, found that MtCLE peptides control nodulation not only systematically, but also locally (Mortier et al., 2010). In this study, 25 MtCLE genes in the Medicago genome have been identified. Two genes, MtCLE12 and MtCLE13, negatively regulate nodulation. MtCLE12 and MtCLE13 have a high degree of similarity with LjCLE-RS1 and LjCLE-RS2 (Mortier et al., 2010). Similarity in sequence and expression profiles suggests that MtCLE13 and $L j C L E-R S 1 / L j C L E-R S 2$ might exert a comparable function, respectively. Studies in Soybean on GmNARK revealed similar results as described for Lotus and Medicago. Gmnark knockout mutants are affected in both AON and nitrate regulation of nodulation suggesting this gene is a common component of both regulatory mechanisms(Reid et al., 2011). Candidate CLE peptides in Soybean that commit symbiotic functions are GmRIC1, GmRIC2, and GmNIC1. Among these, GmNICl is strongly induced by nitrate and possessed the conserved proline residues close to the C-termini, the part similar as found in MtCLE13 and LjCLE-RS1/2 (Okamoto et al., 2009).

CLE-CLAVATA-type LRR-RLK signaling does not function exclusively in symbiosis, but act as signaling module in plant organ development (Okamoto et al., 2009). This holds also for genes that control root nodule formation. For example, an increased number of emerged lateral roots was reported in the Lotus Ljharl mutant under both symbiotic and non-symbiotic conditions (Wopereis et al., 2000). The compact root architecture 2 (Mtcra2) is another Medicago mutant which is affected in a CLAVATA1-like Leucine-Rich Repeat Receptor-Like Kinase (LRR-RLK). Mtcra 2 mutant plants form an increased number of lateral roots with a reduced number of symbiotic nitrogen-fixing nodules (Huault et al., 2014). These finding reveal the related function of the CLE-CLAVATA1 type signaling modules in lateral root development and autoregulation mechanism. 


\section{The role of plant hormones in legume root nodule formation}

Rhizobium root nodule formation is causally linked with formation of a local maximum of indoleacetic acid (IAA), the natural auxin in plants (Mathesius et al., 1998; Pacios-Bras et al., 2003; Huo et al., 2006; van Noorden et al., 2007; Takanashi et al., 2011; Imanishi et al., 2014; Chiu et al., 2005; Perrine-Walker et al., 2010; Suzaki et al., 2012). Based on auxin responsive reporter constructs as well as quantification studies, it is known that IAA is not uniformly distributed in the plant root. The highest concentrations are found in the plant cells undergoing cell divisions -e.g. the root apical meristem and lateral root primordia, whereas also in the elongation zone relative high amounts of auxin are detected (Pacios-Bras et al., 2003; Larkin et al., 1996; Deinum et al., 2012). Auxin is accumulating at the sites of nodule development, which implies that LCO signaling interferes with auxin homeostasis in the plant root. The mechanism by which such local auxin maximum is formed remains unknown; however it is unlikely that LCOs trigger such response cell autonomously. This because LCOs are extremely immobile and stick within walls of epidermal cells, whereas in most legumes the auxin maximum is formed in cortical and pericycle cell layers (Deinum et al., 2012; Marhavý et al., 2013). This suggests that upon perception of LCOs a secondary signal will released from the epidermis that triggers an auxin maximum in lower layers. Quantitative modelling suggests that an auxin maximum can be formed by local inhibition of auxin efflux transport; e.g. by interfering with the functioning the PIN auxin efflux carrier proteins (Deinum et al., 2012). Several compounds are known that have such function; including the plant hormones cytokinin and strigolactones as well as flavonoids. The plant hormone cytokinin is implicated in the regulation of many physiological processes during plant development, growth, and adaptation to environmental conditions (Martín et al., 2000; Mok and Mok, 2001). Active cytokinins accumulate upon LCO signaling (van Zeijl et al., 2015; Frugier et al., 2008; Gonzalez-Rizzo et al., 2006; Lohar et al., 2006; Tirichine et al., 2007). It has been reported that a gain-of-function mutation in the cytokinin receptor LjLHK1 of Lotus and MtCRE1 of Medicago triggers spontaneous root nodule organogenesis (Tirichine et al., 2007; Ovchinnikova et al., 2011). This indicates that perception of the phytohormone cytokinin is a key element in nodule formation in legumes, which is underlined with experiments in which application of exogenous cytokinin induces formation of nodule primordia (Heckmann et al., 2011; Cooper and Long, 1994). In the opposite manner, reduced cytokinin accumulation and/or perception blocks nodulation (Murray et al., 2007; Gonzalez-Rizzo et al., 2006). This 


\section{Chapter 1}

demonstrates unequivocally that cytokinin signaling is necessary and sufficient to induce cortical cell divisions and nodule organogenesis.

Besides cytokinins, also the biosynthetic pathways of strigolactones and flavonoids are activated upon LCO signaling. Both are known to have the capacity to interfere with PIN functioning. However, knockout mutants in strigolactone biosynthesis genes have only a weak symbiotic phenotype, suggesting that strigolactone functioning is less essential for root nodule formation Interestingly, it was found that exogenous application of flavonoids can complement the knockout phenotype of Mtcrel. This suggests that flavonoid act downstream of or in parallel of cytokinins ( $\mathrm{Ng}$ et al., 2015).

Besides hormones with a positive effect on root nodule formation, several hormones have been implicated to have a negative effect on LCO signaling and root nodule formation. For example, abscisic acid and jasmonic acid are known to interfere with LCO induced calcium spiking (Ding et al., 2008; Sun et al., 2006). Also the gaseous plant hormone ethylene is known to have a negative effect on this physiological response, although it is not fully blocking it. Ethylene affects the maintenance of the response, as well as it changes the threshold concentration of LCO required for the induction of calcium spiking (Oldroyd et al., 2001). Further, ethylene acts as inhibitor of nodule formation and bacterial infection. The application of ethylene, or ethylenereleasing compounds, inhibits nodule organogenesis in numerous species including Phaseolus vulgaris (Grobbelaar et al., 1971), Pea (Drennan and Norton, 1972) Trifolium repens (Goodlass and Smith, 1979) Melilotus alba (Lee and LaRue, 1992) Medicago and Lotus (Penmetsa and Cook, 1997; Nukui et al., 2000).

Like cytokinin. Several lines of evidence indicate that ethylene signaling is an integral part in root nodule formation. For example, local biosynthesis of ethylene opposite phloem poles is thought to provide positional information by locally inhibiting root cortical cell divisions (Heidstra et al., 1997). Also there might be a low threshold of ethylene required for proper infection by rhizobium bacteria (Ferguson and Mathesius, 2003; Oldroyd et al., 2001). The negative ethylene effect on nodule number could be accounted for invoking a specific ethylene regulation of infection thread growth (Oldroyd et al., 2001). The role of ethylene in regulation of rhizobial symbiosis is supported with the identification of ethylene signaling mutant ein 2 in Lotus and Medicago (Miyata et al., 2013; Chan et al., 2013; Penmetsa and Cook, 1997). For example, the Medicago Mtein2/sickle knockout mutant makes the plant insensitive to ethylene 
(Penmetsa and Cook, 1997), which dramatically effects nodule formation. In small root zones numerous nodules are formed that have lost spatial positioning in respect the stele of the root. Detailed analysis of the Mtein2/sickle mutant indicated that the read out of rhizobium LCO signaling is stronger. For example, root hair deformation in response to LCOs revealed a stronger response in Mtein2/sickle compared to the wild-type plants (Oldroyd et al., 2001). Ethylene also has an effect on calcium spiking that may regulate the specificity of downstream responses. This effect could be either on the initiation of calcium spiking directly or that ethylene has the capacity to modulate the frequency of calcium spikes (Oldroyd et al., 2001). This shows that ethylene acts upstream or at the point of calcium spiking in the LCO signal transduction pathway, suggesting that it can modulate both the degree and the nature of LCO pathway activation. Furthermore, the LCO concentration threshold that is required to activate calcium spiking is defined by the ethylene status (Oldroyd et al., 2001). It is also known that ethylene signaling regulate auxin transport at certain stages of nodule development. Ethylene partially modulate auxin transporter through PIN gene expression, ultimately an increase in auxin transport results in higher nodule numbers (Prayitno et al., 2006).

Whereas much is known about the molecular dialog of the legume-rhizobium mutualism, it has not been possible so far to answer the question why the rhizobium symbiosis trait is restricted to two lineages; legumes and Parasponia. Genomics studies showed that for most -if not allsymbiosis genes close homologs are present in all plant species that are able to establish an endomycorrhizal symbiosis (Parniske, 2008). To get insight in the evolutionary trajectory of the rhizobium symbiosis trait comparative systems are essential. A comparison of legumes to Parasponia for example may provide insights to what extend genetic constraints have guided evolution of rhizobium $\mathrm{N}_{2}$-fixing root nodules. Studies in this direction revealed that Parasponia nodulates with rhizobium strains that also can form root nodules with legumes (Op den Camp et al., 2012). Like with (most) legumes, the Parasponia-rhizobium symbiosis is driven by LCO signaling (Marvel et al., 1987). Also one LysM-type LCO receptor has been identified, PaNFP, which showed to be orthologous to MtNFP/LjNFR5 of Medicago (den Camp et al., 2011). Furthermore, it was found that an autoactive allele of CCaMK can trigger formation of root nodule-like structures in absence of rhizobium or LCOs (den Camp et al., 2011). Taken together these data suggest that at least in part, both symbioses make use of identical signaling queues. In addition to a Legume-Parasponia comparison to identify genetic constraints, a comparison of 


\section{Chapter 1}

symbiotic plants with non-symbiotic plants may uncover novel insights. For such comparison the plant species that differ in phenotype should be closely related. Furthermore, it is important that the non-nodulating plant species used in such comparison, does not represent a recent loss of the rhizobium symbiosis, but rather never gained it. In the legume family several non-nodulating plant lineages occur, but it remains elusive whether these represent a loss of nodulation or never gained it (Doyle, 2011). In legumes a non-nodulating plant species are represented by Cercideae, which form the most basal lineage in this family (Lavin et al., 2005). This lineage diverged about 59 million years ago from the legume crown node, which represents all nodulating legumes. In contrast, the Parasponia lineage is considered to be much younger than the legume crown node (Behm et al., 2014). Therefore, Parasponia, and its non-nodulating sister species of the genus Trema, may represent the best known comparative system of a nodulating and non-nondulating plant species.

\section{Parasponia-rhizobium symbiosis}

The genus Parasponia belongs to the Cannabaceae (Doyle, 1998). Cannabaceae (order Rosales) and Fabaceae (order Fabales) are only remotely related (figure 1), with a last common ancestor about 100 million years ago (Wang et al., 2009). Therefore it is most probable that both lineages gained the rhizobium-nodule symbiosis independently. 


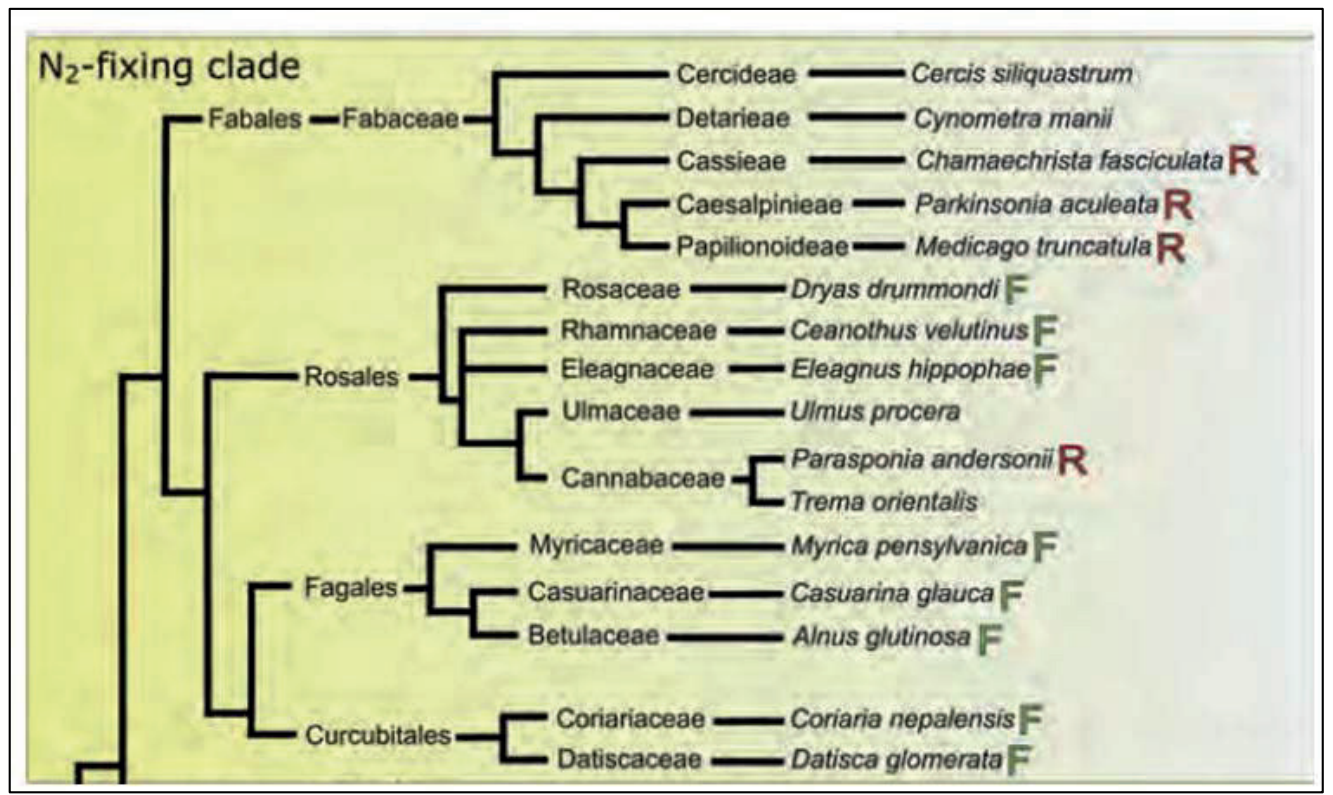

Figure 1. Phylogenetic representation of evolutionary distance between Parasponia and legumes. F: Frankia, R: Rhizobia.

Parasponia root nodules have a more basal appearance when compared to legume nodules. Parasponia nodules have a central vascular bundle with infected cells in the peripheral zone (Cao et al., 2012) and a meristem at the tip. Cells proximal of this meristem are being invaded by rhizobia upon formation of infection threads. In contrast to most legume nodules the bacteria are not released into the cell as symbiosomes, but remain within thread-like structures. These, socalled fixation threads, differ from the penetrating infection threads in such a way that they have a cell wall that is significantly reduced in thickness. Also, fixation threads are somewhat wider than infection threads, allowing formation of double phyla of nitrogen-fixing bacteria. In contrast, in most legume nodules infection threads release rhizobia from the tip of the thread into the cytoplasm. At this point the host cell encloses the newly-released bacteria within a cell membrane, so that it is not actually in contact with the plant cytoplasm. These bacteria then differentiate into their symbiotic $\mathrm{N}_{2}$-fixing organelle-like structures, and effectively become dependent on the host plant.

The structure of fixation threads somewhat resembles the structure of arbuscules formed by AM 


\section{Chapter 1}

fungi in the root cortical cells of their host plant. Overlap in both structures is support with the finding that PaNFP controls formation of both (den Camp et al., 2011). Fixation threads are not unique to Parasponia. Several basal legumes host their symbiotic microbe in a similar way. For example, Andira as well as some Chamaecrista species form also fixation threads (also named persistent infection threads) (Faria et al., 1987; Naisbitt et al., 1992). Andira and Chamaecrista do not represent closely related legume lineages, but diverged $\sim 58$ million years ago. Fixation thread containing nodules harbor significantly less bacteria per cell when compared to nodules with symbiosomes (Behm et al., 2014). Additionally, symbiosomes are deprived from cell wall material and have an enlarged surface of surrounding membrane when compared to fixation thread, which may be an advantage in nutrient exchange between both partners. Taken together it seems most probable that fixation threads resemble a more ancestral stage of intracellular rhizobium infection (de Faria et al., 1986; Naisbitt et al., 1992).

The Parasponia-rhizobium symbiosis is more basal when compared to the legume-rhizobium symbiosis. Not only the nodules are more basal in structure when compared to legumes, also the nitrogen fixating capacity is lower than in legumes by a $0.5-1.0$ order of magnitude (Vessey et al., 2004). The primitive nature of Parasponia root nodules is further underlined by the mode of rhizobium infection of the root. Infection of Parasponia by rhizobia occur by intercellular penetration of the root; a mechanism known as crack entry. Such mode of infection is lesssophisticated than root hair based intracellular infection as found in most legume species. Coinciding with crack entry is partner promiscuity in Parasponia, which allows nodulation by many rhizobial species, irrespective of their nitrogen fixation capacities (Op den Camp et al., 2012).

Taken together, I argue that due to its young age and independent evolution, Parasponia provides a simple system to identify the genetic basis underlying rhizobium symbiosis evolution. Thereby the main question of my research is how this mutualistic interaction evolved in Parasponia, and which constraints have guided this evolutionary trajectory?

\section{Forward evolution in Trema}

Parasponia represents 5 tropical tree species that can be found on volcanic regions in the Malay archipelago. These species are phenotypically very similar to non-nodulating Trema species, which occasionally leads to incorrect species determination (Akkermans et al., 1978). Although 
Parasponia and Trema can be distinguished by some phenotypic features like their imbricate perianth lobes of the male flowers and by their intrapetiolar (Trinick, 1973). Recent phylogenetic studies using chloroplast genes such as $r b c L$, and $\operatorname{trn} L$ or the internal transcribed spacer region of 18S-26S nuclear ribosomal DNA as markers, indicated that the Parasponia lineages is nested in the Trema genus (Yang et al., 2013). This suggests that Parasponia evolved only relatively recent, including its symbiotic capacities, from an ancestral Trema species. Such hypothesis is supported by the more restricted distribution of Parasponia when compared to Trema. The genus Trema includes 15 species that can be found in Asia, Australia, Africa and the Americas. Ever since the discovery of Parasponia (in 1973) as the first, and till now only, non-legume that independently evolved the nitrogen-fixing nodule symbiosis with rhizobium, it intrigued the scientific community. Comparing Parasponia not only to legumes, but also to Trema can help to characterize the genetics of the nitrogen fixing rhizobium root nodule trait.

\section{Scope and outline of the thesis}

The overall aim of this research was to identify the evolutionary trajectory by which Parasponia became able to establish the rhizobium nodule symbiosis. To investigate the nodulation process as a specific difference between Parasponia and Trema, a collection of techniques was needed, including plant transformation protocols. In chapter 2, I described a rapid Agrobacterium rhizogenes-mediated transformation method to generate composite plants carrying transgenic root for Parasponia andersonii and Trema tomentosa. This 'transient' transformation system allowed functional testing of transgenes. Unfortunately, this transient method was not very efficient for Trema sp., which forced me to develop a novel protocol for stable -A. tumefaciensmediated transformation of $T$. tomentosa (chapter 3). By this protocol, T. tomentosa plants carrying DR5::GUS were generated within a period of 6 months. Subsequently, the transgenic T0 lines were vegetatively propagated to study the DR5::GUS expression.

In chapter 4, 5 and 6 I studied the biology of the Parasponia-rhizobium symbiosis. Along the idea that Parasponia-rhizobium symbiosis evolved recently, I questioned whether Parasponia has gained mechanisms to control the interaction with its symbiotic partner. In this context I tested whether Parasponia can control root nodule formation in presence of an exogenous fixed nitrogen source (chapter 4). Considering that Parasponia is a tree with higher nitrate demand, I questioned which exogenous nitrate concentration will affect the nitrogen fixing symbiosis in 


\section{Chapter 1}

Parasponia? To do so, I examined the effect of a range of nitrate treatments on the rhizobium induced symbiotic responses in Parasponia. This revealed that nodule primordium formation and intracellular infection have different sensitivities to nitrates. Formation of nodules was optimal at $10 \mathrm{mM}$ nitrate, whereas progression of intracellular infection was impaired at $20 \mathrm{mM}$ nitrate. Specifically, the switch from infection to fixation thread formation was affected by exogenous nitrate. In line with this finding I argued that this effect of exogenous nitrate in the rhizobium symbiosis, is a novel invention in Parasponia.

Next I investigated whether the GRAS-type transcription factors NSP1 and NSP2 (Chapter 5) and ethylene signaling (Chapter 6), like in legumes, are co-opted by Parasponia to control root nodule formation. To do so, Parasponia orthologous genes to Medicago NSP1, NSP2 and EIN2 have been cloned and studied by RNAi using A. rhizogenes mediated transformation. This revealed that all these genes commit a symbiotic role, supporting the hypothesis that genetic constrains have guided evolution of rhizobium symbiosis in legumes and Parasponia.

In Chapter 7 (General discussion) I place these findings in a broader context. 


\section{General Introduction}

\section{References}

Akkermans, A., et al. (1978). "N2-fixing root nodules in Ulmaceae: Parasponia or (and) Trema spp.?" Plant and Soil 49(3): 711-715.

Ané, J.-M., et al. (2004). "Medicago truncatula DMI1 required for bacterial and fungal symbioses in legumes." Science 303(5662): 1364-1367.

Arrighi, J.-F., et al. (2006). "The Medicago truncatula lysine motif-receptor-like kinase gene family includes NFP and new nodule-expressed genes." Plant Physiology 142(1): 265-279.

Barbulova, A., et al. (2007). "Differential effects of combined N sources on early steps of the Nod factor-dependent transduction pathway in Lotus japonicus." Molecular Plant-Microbe Interactions 20(8): 994-1003.

Behm, J. E., et al. (2014). "Parasponia: a novel system for studying mutualism stability." Trends in Plant Science 19(12): 757-763.

Bravo, A., et al. (2016). "Genes conserved for arbuscular mycorrhizal symbiosis identified through phylogenomics." Nature Plants 2: 15208

Broghammer, A., et al. (2012). "Legume receptors perceive the rhizobial lipochitin oligosaccharide signal molecules by direct binding." Proceedings of the National Academy of Sciences 109(34): 13859-13864.

Buendia, L., et al. (2015). "The LysM receptor-like kinase SILYK10 regulates the arbuscular mycorrhizal symbiosis in tomato." New Phytologist.

Cao, Q., et al. (2012). "Efficiency of Agrobacterium rhizogenes-mediated root transformation of Parasponia and Trema is temperature dependent." Plant Growth Regulation 68(3): 459-465.

Carroll, B. J., et al. (1985). "A supernodulation and nitrate-tolerant symbiotic (nts) soybean mutant." Plant Physiology 78(1): 34-40.

Cerri, M. R., et al. (2016). "The Symbiosis-Related ERN Transcription Factors Act in Concert to Coordinate Rhizobial Host Root Infection." Plant Physiology: pp. 00230.02016.

Cerri, M. R., et al. (2012). "Medicago truncatula ERN transcription factors: regulatory interplay with NSP1/NSP2 GRAS factors and expression dynamics throughout rhizobial infection." Plant Physiology 160(4): 2155-2172.

Chabaud, M., et al. (2016). "Chitinase-resistant hydrophilic symbiotic factors secreted by Frankia activate both $\mathrm{Ca} 2+$ spiking and NIN gene expression in the actinorhizal plant Casuarina glauca." New Phytologist 209(1): 86-93.

Chan, P. K., et al. (2013). "Classical Ethylene Insensitive Mutants of the Arabidopsis EIN2 Orthologue Lack the Expected 'hypernodulation'Response in Lotus japonicusF." Journal of Integrative Plant Biology 55(4): 395-408.

Chen, C., et al. (2008). "OsIPD3, an ortholog of the Medicago truncatula DMI3 interacting protein IPD3, is required for mycorrhizal symbiosis in rice." New Phytologist 180(2): 311-315.

Chen, T., et al. (2012). "A MAP kinase kinase interacts with SymRK and regulates nodule organogenesis in Lotus japonicus." The Plant cell 24(2): 823-838.

Chiu, W.-L., et al. (2005). "Nitrogen deprivation stimulates symbiotic gland development in Gunnera manicata." Plant Physiology 139(1): 224-230.

Clavijo, F., et al. (2015). "The Casuarina NIN gene is transcriptionally activated throughout Frankia root infection as well as in response to bacterial diffusible signals." New Phytologist 208(3): 887-903. 


\section{Chapter 1}

Cooper, J. B. and S. R. Long (1994). "Morphogenetic rescue of Rhizobium meliloti nodulation mutants by transzeatin secretion." The Plant cell 6(2): 215-225.

D’Haeze, W. and M. Holsters (2002). "Nod factor structures, responses, and perception during initiation of nodule development." Glycobiology 12(6): 79R-105R.

de Faria, S. M., et al. (1986). "A new type of infected cell in root nodules of Andira spp.(Leguminosae)." Plant Science 45(2): 143-147.

Deinum, E. E., et al. (2012). "Modeling a cortical auxin maximum for nodulation: different signatures of potential strategies." Front Plant Sci 3: 96.

Delaux, P.-M., et al. (2014). "Comparative phylogenomics uncovers the impact of symbiotic associations on host genome evolution." PLoS Genet 10(7): e1004487.

Delaux, P. M., et al. (2013). "NSP1 is a component of the Myc signaling pathway." New Phytologist 199(1): 59-65.

den Camp, R. O., et al. (2011). "LysM-type mycorrhizal receptor recruited for rhizobium symbiosis in nonlegume Parasponia." Science 331(6019): 909-912.

Diédhiou, I., et al. (2014). "Identification of potential transcriptional regulators of actinorhizal symbioses in Casuarina glauca and Alnus glutinosa." BMC plant biology 14(1): 1.

Ding, Y., et al. (2008). "Abscisic acid coordinates nod factor and cytokinin signaling during the regulation of nodulation in Medicago truncatula." The Plant cell 20(10): 2681-2695.

Doyle, J. J. (1998). "Phylogenetic perspectives on nodulation: evolving views of plants and symbiotic bacteria." Trends in Plant Science 3(12): 473-478.

Doyle, J. J. (2011). "Phylogenetic perspectives on the origins of nodulation." Molecular Plant-Microbe Interactions 24(11): 1289-1295.

Drennan, D. and C. Norton (1972). "The effect of ethrel on nodulation inPisum sativum L." Plant and Soil 36(1-3): 53-57.

Elise, S., et al. (2005). "The Medicago truncatula SUNN gene encodes a CLV1-like leucine-rich repeat receptor kinase that regulates nodule number and root length." Plant Molecular Biology 58(6): 809-822.

Endre, G., et al. (2002). "A receptor kinase gene regulating symbiotic nodule development." Nature 417(6892): 962966.

Faria, S. M. d., et al. (1987). "The occurrence of infected cells, with persistent infection threads, in legume root nodules." Canadian Journal of Botany 65(3): 553-558.

Ferguson, B. J. and U. Mathesius (2003). "Signaling interactions during nodule development." Journal of Plant Growth Regulation 22(1): 47-72.

Frugier, F., et al. (2008). "Cytokinin: secret agent of symbiosis." Trends in Plant Science 13(3): 115-120.

Geurts, R., et al. (2005). "Nod factor signaling genes and their function in the early stages of Rhizobium infection." Current Opinion in Plant Biology 8(4): 346-352.

Geurts, R. and V. G. Vleeshouwers (2012). "Mycorrhizal symbiosis: ancient signalling mechanisms co-opted." Current Biology 22(23): R997-R999. 


\section{General Introduction}

Geurts, R., et al. (2016). "What Does It Take to Evolve A Nitrogen-Fixing Endosymbiosis?" Trends in Plant Science 21(3): 199-208.

Gherbi, H., et al. (2008). "SymRK defines a common genetic basis for plant root endosymbioses with arbuscular mycorrhiza fungi, rhizobia, and Frankiabacteria." Proceedings of the National Academy of Sciences 105(12): 49284932.

Gonzalez-Rizzo, S., et al. (2006). "The Medicago truncatula CRE1 cytokinin receptor regulates lateral root development and early symbiotic interaction with Sinorhizobium meliloti." The Plant cell 18(10): 2680-2693.

Goodlass, G. and K. Smith (1979). "Effects of ethylene on root extension and nodulation of pea (\&lt;i\&gt;Pisum sativum\&lt;/i\&gt; L.) and white clover (\&lt;i\&gt;Trifolium repens\&lt;/i\&gt; L.)." Plant and Soil 51(3): 387-395.

Gough, C. and J. Cullimore (2011). "Lipo-chitooligosaccharide signaling in endosymbiotic plant-microbe interactions." Molecular Plant-Microbe Interactions 24(8): 867-878.

Granqvist, E., et al. (2015). "Bacterial-induced calcium oscillations are common to nitrogen-fixing associations of nodulating legumes and non-legumes." New Phytologist 207(3): 551-558.

Grobbelaar, N., et al. (1971). "The nodulation and nitrogen fixation of isolated roots of Phaseolus vulgaris L." Plant and Soil 35(1): 203-214.

Groth, M., et al. (2010). "NENA, a Lotus japonicus homolog of Sec13, is required for rhizodermal infection by arbuscular mycorrhiza fungi and rhizobia but dispensable for cortical endosymbiotic development." The Plant cell 22(7): 2509-2526.

Gutjahr, C. and M. Parniske (2013). "Cell and developmental biology of arbuscular mycorrhiza symbiosis."

Heckmann, A. B., et al. (2006). "Lotus japonicus nodulation requires two GRAS domain regulators, one of which is functionally conserved in a non-legume." Plant Physiology 142(4): 1739-1750.

Heckmann, A. B., et al. (2011). "Cytokinin induction of root nodule primordia in Lotus japonicus is regulated by a mechanism operating in the root cortex." Molecular Plant-Microbe Interactions 24(11): 1385-1395.

Heidstra, R., et al. (1997). "Ethylene provides positional information on cortical cell division but is not involved in Nod factor-induced root hair tip growth in Rhizobium-legume interaction." Development 124(9): 1781-1787.

Hirakawa, Y., et al. (2008). "Non-cell-autonomous control of vascular stem cell fate by a CLE peptide/receptor system." Proceedings of the National Academy of Sciences 105(39): 15208-15213.

Hirel, B., et al. (2007). "The challenge of improving nitrogen use efficiency in crop plants: towards a more central role for genetic variability and quantitative genetics within integrated approaches." Journal of Experimental Botany 58(9): 2369-2387.

Hirsch, S., et al. (2009). "GRAS proteins form a DNA binding complex to induce gene expression during nodulation signaling in Medicago truncatula." The Plant cell 21(2): 545-557.

Huault, E., et al. (2014). "Local and systemic regulation of plant root system architecture and symbiotic nodulation by a receptor-like kinase." PLoS Genet 10(12): e1004891.

Huo, X., et al. (2006). "RNAi phenotypes and the localization of a protein:: GUS fusion imply a role for Medicago truncatula PIN genes in nodulation." Journal of Plant Growth Regulation 25(2): 156-165.

Imaizumi-Anraku, H., et al. (2005). "Plastid proteins crucial for symbiotic fungal and bacterial entry into plant roots." Nature 433(7025): 527-531. 


\section{Chapter 1}

Imanishi, L., et al. (2014). "Role of auxin during intercellular infection of Discaria trinervis by Frankia." Frontiers in plant science 5.

Ivanov, S., et al. (2012). "Rhizobium-legume symbiosis shares an exocytotic pathway required for arbuscule formation." Proceedings of the National Academy of Sciences 109(21): 8316-8321.

Kahle, J., et al. (2005). "Subunits of the heterotrimeric transcription factor NF-Y are imported into the nucleus by distinct pathways involving importin $\beta$ and importin 13." Molecular and Cellular Biology 25(13): 5339-5354.

Kanamori, N., et al. (2006). "A nucleoporin is required for induction of Ca2+ spiking in legume nodule development and essential for rhizobial and fungal symbiosis." Proceedings of the National Academy of Sciences of the United States of America 103(2): 359-364.

Kawaguchi, M., et al. (2002). "Root, root hair, and symbiotic mutants of the model legume Lotus japonicus." Molecular Plant-Microbe Interactions 15(1): 17-26.

Kistner, C. and M. Parniske (2002). "Evolution of signal transduction in intracellular symbiosis." Trends in Plant Science 7(11): 511-518.

Kistner, C., et al. (2005). "Seven Lotus japonicus genes required for transcriptional reprogramming of the root during fungal and bacterial symbiosis." The Plant cell 17(8): 2217-2229.

Larkin, P., et al. (1996). "Transgenic white clover. Studies with the auxin-responsive promoter, GH3, in root gravitropism and lateral root development." Transgenic research 5(5): 325-335.

Lavin, M., et al. (2005). "Evolutionary rates analysis of Leguminosae implicates a rapid diversification of lineages during the Tertiary." Systematic biology 54(4): 575-594.

Lee, K. H. and T. A. LaRue (1992). "Exogenous ethylene inhibits nodulation of Pisum sativum L. cv Sparkle." Plant Physiology 100(4): 1759-1763.

Lévy, J., et al. (2004). "A putative Ca2+ and calmodulin-dependent protein kinase required for bacterial and fungal symbioses." Science 303(5662): 1361-1364.

Limpens, E., et al. (2009). "Medicago N2-fixing symbiosomes acquire the endocytic identity marker Rab7 but delay the acquisition of vacuolar identity." The Plant cell 21(9): 2811-2828.

Liu, W., et al. (2011). "Strigolactone biosynthesis in Medicago truncatula and rice requires the symbiotic GRAStype transcription factors NSP1 and NSP2." The Plant cell 23(10): 3853-3865.

Lohar, D. P., et al. (2006). "Transcript analysis of early nodulation events in Medicago truncatula." Plant Physiology 140(1): 221-234.

Lum, M. R. and A. M. Hirsch (2002). "Roots and their symbiotic microbes: strategies to obtain nitrogen and phosphorus in a nutrient-limiting environment." Journal of Plant Growth Regulation 21(4): 368-382.

Madsen, E. B., et al. (2011). "Autophosphorylation is essential for the in vivo function of the Lotus japonicus Nod factor receptor 1 and receptor-mediated signalling in cooperation with Nod factor receptor 5." The Plant Journal 65(3): 404-417.

Madsen, E. B., et al. (2003). "A receptor kinase gene of the LysM type is involved in legumeperception of rhizobial signals." Nature 425(6958): 637-640.

Magori, S., et al. (2009). "TOO MUCH LOVE, a root regulator associated with the long-distance control of nodulation in Lotus japonicus." Molecular Plant-Microbe Interactions 22(3): 259-268. 


\section{General Introduction}

Maillet, F., et al. (2011). "Fungal lipochitooligosaccharide symbiotic signals in arbuscular mycorrhiza." Nature 469(7328): 58-63.

Malkov, N., et al. (2016). "Molecular basis of lipo-chitooligosaccharide recognition by the lysin motif receptor-like kinase LYR3 in legumes." Biochemical Journal 473(10): 1369-1378.

Marhavý, P., et al. (2013). "Auxin reflux between the endodermis and pericycle promotes lateral root initiation." The EMBO journal 32(1): 149-158.

Markmann, K., et al. (2008). "Functional adaptation of a plant receptor-kinase paved the way for the evolution of intracellular root symbioses with bacteria." PLoS Biol 6(3): e68.

Marsh, J. F., et al. (2007). "Medicago truncatula NIN is essential for rhizobial-independent nodule organogenesis induced by autoactive calcium/calmodulin-dependent protein kinase." Plant Physiology 144(1): 324-335.

Martín, A. C., et al. (2000). "Influence of cytokinins on the expression of phosphate starvation responsive genes in Arabidopsis." The Plant Journal 24(5): 559-567.

Martínez-Romero, E. (2009). "Coevolution in Rhizobium-legume symbiosis?" DNA and cell biology 28(8): 361370 .

Marvel, D. J., et al. (1987). "Rhizobium symbiotic genes required for nodulation of legume and nonlegume hosts." Proceedings of the National Academy of Sciences 84(5): 1319-1323.

Mathesius, U., et al. (1998). "Auxin transport inhibition precedes root nodule formation in white clover roots and is regulated by flavonoids and derivatives of chitin oligosaccharides." The Plant Journal 14(1): 23-34.

Messinese, E., et al. (2007). "A novel nuclear protein interacts with the symbiotic DMI3 calcium-and calmodulindependent protein kinase of Medicago truncatula." Molecular Plant-Microbe Interactions 20(8): 912-921.

Mitra, R. M., et al. (2004). "A Ca2+/calmodulin-dependent protein kinase required for symbiotic nodule development: gene identification by transcript-based cloning." Proceedings of the National Academy of Sciences of the United States of America 101(13): 4701-4705.

Miyata, K., et al. (2013). "Two distinct EIN2 genes cooperatively regulate ethylene signaling in Lotus japonicus." Plant and Cell Physiology 54(9): 1469-1477.

Mok, D. W. and M. C. Mok (2001). "Cytokinin metabolism and action." Annual review of plant biology 52(1): 89118.

Mortier, V., et al. (2010). "CLE peptides control Medicago truncatula nodulation locally and systemically." Plant Physiology 153(1): 222-237.

Murray, J. D., et al. (2007). "A cytokinin perception mutant colonized by Rhizobium in the absence of nodule organogenesis." Science 315(5808): 101-104.

Naisbitt, T., et al. (1992). "The evolutionary significance of the legume genus Chamaecrista, as determined by nodule structure." New Phytologist 122(3): 487-492.

Nap, J.-P. and T. Bisseling (1990). "Developmental biology of a plant-prokaryote symbiosis: the legume root nodule." Science(Washington) 250(4983): 948-954.

Ng, J. L. P., et al. (2015). "Flavonoids and auxin transport inhibitors rescue symbiotic nodulation in the Medicago truncatula cytokinin perception mutant cre1." The Plant cell 27(8): 2210-2226. 


\section{Chapter 1}

Nukui, N., et al. (2000). "Effects of ethylene precursor and inhibitors for ethylene biosynthesis and perception on nodulation in Lotus japonicus and Macroptilium atropurpureum." Plant and Cell Physiology 41(7): 893-897.

Okamoto, S. and M. Kawaguchi (2015). "Shoot HAR1 mediates nitrate inhibition of nodulation in Lotus japonicus." Plant Signaling \& Behavior 10(5): e1000138.

Okamoto, S., et al. (2009). "Nod factor/nitrate-induced CLE genes that drive HAR1-mediated systemic regulation of nodulation." Plant and Cell Physiology 50(1): 67-77.

Okamoto, S., et al. (2013). "Root-derived CLE glycopeptides control nodulation by direct binding to HAR1 receptor kinase." Nature communications 4.

Oldroyd, G. E., et al. (2001). "Ethylene inhibits the Nod factor signal transduction pathway of Medicago truncatula." The Plant cell 13(8): 1835-1849.

Olsson, J. E., et al. (1989). "Lack of systemic suppression of nodulation in split root systems of supernodulating soybean (Glycine max [L.] Merr.) mutants." Plant Physiology 90(4): 1347-1352.

Op den Camp, R. H., et al. (2012). "Nonlegume Parasponia andersonii deploys a broad rhizobium host range strategy resulting in largely variable symbiotic effectiveness." Molecular Plant-Microbe Interactions 25(7): 954-963.

Ovchinnikova, E., et al. (2011). "IPD3 controls the formation of nitrogen-fixing symbiosomes in pea and Medicago Spp." Molecular Plant-Microbe Interactions 24(11): 1333-1344.

Pacios-Bras, C., et al. (2003). "Auxin distribution in Lotus japonicus during root nodule development." Plant Molecular Biology 52(6): 1169-1180.

Parniske, M. (2008). "Arbuscular mycorrhiza: the mother of plant root endosymbioses." Nature Reviews Microbiology 6(10): 763-775.

Penmetsa, R. V. and D. R. Cook (1997). "A legume ethylene-insensitive mutant hyperinfected by its rhizobial symbiont." Science 275(5299): 527-530.

Perrine-Walker, F., et al. (2010). "Auxin carriers localization drives auxin accumulation in plant cells infected by Frankia in Casuarina glauca actinorhizal nodules." Plant Physiology 154(3): 1372-1380.

Pietraszewska-Bogiel, A., et al. (2013). "Interaction of Medicago truncatula lysin motif receptor-like kinases, NFP and LYK3, produced in Nicotiana benthamiana induces defence-like responses." PLoS ONE 8(6): e65055.

Prayitno, J., et al. (2006). "The ethylene-insensitive sickle mutant of Medicago truncatula shows altered auxin transport regulation during nodulation." Plant Physiology 142(1): 168-180.

Read, D., et al. (2000). "Symbiotic fungal associations in 'lower'land plants." Philosophical Transactions of the Royal Society of London B: Biological Sciences 355(1398): 815-831.

Reid, D. E., et al. (2011). "Inoculation-and nitrate-induced CLE peptides of soybean control NARK-dependent nodule formation." Molecular Plant-Microbe Interactions 24(5): 606-618.

Reid, D. E., et al. (2011). "Molecular mechanisms controlling legume autoregulation of nodulation." Annals of Botany 108(5): 789-795.

Roth, L. and G. Stacey (1989). "Bacterium release into host cells of nitrogen-fixing soybean nodules: the symbiosome membrane comes from three sources." European journal of cell biology 49(1): 13-23.

Saito, K., et al. (2007). "NUCLEOPORIN85 is required for calcium spiking, fungal and bacterial symbioses, and seed production in Lotus japonicus." The Plant cell 19(2): 610-624. 


\section{General Introduction}

Schauser, L., et al. (1999). "A plant regulator controlling development of symbiotic root nodules." Nature 402(6758): 191-195.

Schnabel, E., et al. (2011). "ROOT DETERMINED NODULATION 1 regulates nodule number in M. truncatula and defines a highly conserved, uncharacterized plant gene family." Plant Physiology: pp. 111.178756.

Singh, S., et al. (2014). "CYCLOPS, a DNA-binding transcriptional activator, orchestrates symbiotic root nodule development." Cell Host \& Microbe 15(2): 139-152.

Soyano, T., et al. (2013). "Nodule inception directly targets NF-Y subunit genes to regulate essential processes of root nodule development in Lotus japonicus." PLoS Genet 9(3): e1003352.

Sprent, J. I. (2008). "60Ma of legume nodulation. What's new? What's changing?" Journal of Experimental Botany 59(5): 1081-1084.

Steen, A., et al. (2005). "AcmA of Lactococcus lactis is an $\mathrm{N}$-acetylglucosaminidase with an optimal number of LysM domains for proper functioning." FEBS Journal 272(11): 2854-2868.

Stracke, S., et al. (2002). "A plant receptor-like kinase required for both bacterial and fungal symbiosis." Nature 417(6892): 959-962.

Streeter, J. and P. P. Wong (1988). "Inhibition of legume nodule formation and N2 fixation by nitrate." Critical Reviews in Plant Sciences 7(1): 1-23.

Sun, J., et al. (2006). "Crosstalk between jasmonic acid, ethylene and Nod factor signaling allows integration of diverse inputs for regulation of nodulation." The Plant Journal 46(6): 961-970.

Suzaki, T., et al. (2012). "Positive and negative regulation of cortical cell division during root nodule development in Lotus japonicus is accompanied by auxin response." Development 139(21): 3997-4006.

Takanashi, K., et al. (2011). "Involvement of auxin distribution in root nodule development of Lotus japonicus." Planta 234(1): 73-81.

Takeda, N., et al. (2013). "CERBERUS and NSP1 of Lotus japonicus are common symbiosis genes that modulate arbuscular mycorrhiza development." Plant and Cell Physiology 54(10): 1711-1723.

Tirichine, L., et al. (2006). "Deregulation of a Ca2\&plus;/calmodulin-dependent kinase leads to spontaneous nodule development." Nature 441(7097): 1153-1156.

Tirichine, L., et al. (2007). "A gain-of-function mutation in a cytokinin receptor triggers spontaneous root nodule organogenesis." Science 315(5808): 104-107.

Trinick, M. (1973). "Symbiosis between Rhizobium and the non-legume, Trema aspera." Nature 244: 459-460. van Noorden, G. E., et al. (2007). "Overlap of proteome changes in Medicago truncatula in response to auxin and Sinorhizobium meliloti." Plant Physiology 144(2): 1115-1131.

van Zeijl, A., et al. (2015). "Rhizobium lipo-chitooligosaccharide signaling triggers accumulation of cytokinins in Medicago truncatula roots." Molecular Plant 8(8): 1213-1226.

Vance, C. P. (2001). "Symbiotic nitrogen fixation and phosphorus acquisition. Plant nutrition in a world of declining renewable resources." Plant Physiology 127(2): 390-397.

Vessey, J. K., et al. (2004). "Root-based N-2-fixing symbioses: Legumes, actinorhizal plants, Parasponia sp and cycads." Plant and Soil 266(1-2): 205-230. 


\section{Chapter 1}

Wang, H., et al. (2009). "Rosid radiation and the rapid rise of angiosperm-dominated forests." Proceedings of the National Academy of Sciences 106(10): 3853-3858.

Wopereis, J., et al. (2000). "Short root mutant of Lotus japonicus with a dramatically altered symbiotic phenotype." The Plant Journal 23(1): 97-114.

Xiao, T. T., et al. (2014). "Fate map of Medicago truncatula root nodules." Development 141(18): 3517-3528. Yang, M.-Q., et al. (2013). "Molecular phylogenetics and character evolution of Cannabaceae." Taxon 62(3): 473485 .

Yano, K., et al. (2008). "CYCLOPS, a mediator of symbiotic intracellular accommodation." Proceedings of the National Academy of Sciences 105(51): 20540-20545. 


\section{Chapter2}

Efficiency of Agrobacterium rhizogenes-Mediated Root Transformation of Parasponia and Trema Is Temperature Dependent

Maryam Seifi Kalhor

Adopted version from: Cao, Q., et al. (2012) Plant Growth Regulation 68(3): 459-465. 


\section{Chapter 2}

\section{Abstract}

Parasponia trees are the only non-legume species that form nitrogen-fixing root nodules with rhizobium. Based on its taxonomic position in relation to legumes (Fabaceae), it is most likely that both lineages have gained this symbiotic capacity independently. Therefore, Parasponia forms a bridging species to understand the evolutionary constraints underlying this symbiosis. However, absence of key technologies to genetically modified Parasponia seriously impeded studies on these species. We employed Agrobacterium rhizogenes to create composite Parasponia andersonii plants that harbor transgenic roots. Here, we provide an optimized protocol to infect $P$. andersonii as well as its non- symbiotic sister species Trema tomentosa with A. rhizogenes. We show that the transformation efficiency is temperature dependent. Whereas the optimal growth temperature for both species is $28^{\circ} \mathrm{C}$, the transformation is most efficient when cocultivation with $A$. rhizogenes occurs at $21^{\circ} \mathrm{C}$. By using of this optimized protocol up to $80 \%$ transformation efficiency can be obtained. These robust transformation platforms will provide a strong tool to unravel the Parasponia-rhizobium symbiosis.

Keywords: Parasponia, Trema, Agrobacterium transformation, Symbiosis, Transgenic root, Composite plant. 


\section{Temperature dependency of Agrobacterium rhizogenes-mediated root}

transformation

\section{Introduction}

Legumes (Fabaceae) are known for their protein-richness and many legume crops are cultivated to provide a protein source for humans. Legume crops have been used in ancient agriculture; e.g. in Neolithic China soybean was grown next to millet (Lee et al., 2007; Guo et al., 2010). Legumes can accumulate such high protein content in leaves and seeds due to a unique endosymbiosis with soil born nitrogen-fixing rhizobium bacteria. This symbiosis results in formation of novel root organs, the so- called root nodules. In nodules, rhizobium is hosted intracellularly and produces ammonia from atmospheric $\mathrm{N}_{2}$. This is the most important biological process by which fixed nitrogen is produced in agriculture (Dawson and Hilton, 2011). The very high efficiency of $\mathrm{N}_{2}$-fixation in legume nodules makes it an already long lasting goal for researchers to transfer this symbiotic capacity to important non-legume crops like rice and wheat (Burrill and Hansen, 1917; Beatty and Good, 2011).

Outside the Fabaceae, there is only a single plant genus, Parasponia, which can establish a similar symbiosis with rhizobium. Parasponia belongs to the Celtidaceae (order Rosales) (Yesson et al., 2004), but molecular phylogenetic studies combine this (sub) family with Cannabaceae and Urticaceae, resulting in a new (super) family of Cannabaceae (Sytsma et al., 2002; II, 2003). Parasponia and Fabaceae are only remotely related and had a last common ancestor 100 million years ago (Wang et al., 2009). This suggests that both lineages evolved rhizobium symbiosis independently. Such independent evolutionary events provide a unique tool to unravel the molecular evolutionary mechanisms underlying the rise of endosymbiosis with rhizobium. Furthermore, studies on Parasponia can teach us how to transfer this important agricultural trait to non- legume crops. However, absence of key technologies to genetically modify Parasponia plants seriously has impeded studies in such directions.

In the Parasponia genus only 5 species are recognized, all of which are tropical trees with their native distribution in the Malay Archipelago, including Indonesia, Malaysia and Papua New Guinea (Trinick, 1973; Akkermans et al., 1978; Becking, 1992). Upon initial discovery it was misclassified and named Trema due to strong phenotypic resemblance to these species, which 


\section{Chapter 2}

are unable to engage a rhizobium symbiosis (Trinick, 1973). The close relation of Trema and Parasponia genera is supported by molecular phylogenetic studies using the chloroplast genes $r b c L$, and $\operatorname{trnL}$ or the internal transcribed spacer (ITS) region of $18 \mathrm{~S}-26 \mathrm{~S}$ nuclear ribosomal DNA as markers (Sytsma et al., 2002; Yesson et al., 2004). In these studies insufficient resolution is obtained to discriminate Parasponia as a monophyletic group from non-symbiotic Trema species. As Parasponia is the only non-legume species able to establish a symbiosis with rhizobium, it suggests that it has gained this capacity relatively recent, most likely just after the split from Trema.

The relative young age of the Parasponia-rhizobium symbiosis is further supported by the rather primitive nature of the root nodules. Parasponia nodules have the appearance of modified lateral roots with a central vascular bundle and infected cells in the peripheral zone (Trinick, 1979). Furthermore, the infection process is also rather primitive. Rhizobium enters the Parasponia root intercellularly by crack entry and only when bacteria reach a nodule primordium, intracellular infection occurs (Becking, 1992). Once inside a cell, the infection thread will branch and the newly formed threads have a very thin cell wall. These so-called fixation threads are filled with rhizobia that can fix nitrogen (Trinick, 1979) (Fig. 1c). Fixation threads resemble the endomembrane compartments containing highly branched hyphae formed by arbuscular mycorrhiza (AM) fungi, called arbuscules. Both structures form a continuum with the plasma membrane. Recent studies in $P$. andersonii revealed that both, AM arbuscules and rhizobium fixation threads, require the LysM-type receptor kinase PaNFP that in legumes is known to function as receptor for rhizobium secreted lipochitooligosaccharides, named Nod factors (den Camp et al., 2011). In legumes this receptor is not essential for mycorrhization, possibly due to subneofunctionalization after a gene duplication event (Young et al., 2011). These data are well in line with the idea that the Parasponia-rhizobium symbiosis is relatively young, and it clearly illustrates that Parasponia has the potential to provide insight in the core mechanisms controlling symbiosis (Streng et al., 2011).

Genetic studies revealed that the evolutionary constraints underlying a rhizobium nodule symbiosis are largely based on the signaling machinery essential for mycorrhization. As the vast majority of plants can establish the endosymbiosis with endomycorrhizal fungi, it suggests that the genetic machinery to establish a rhizobium nodule symbiosis is in principle widespread in the plant kingdom. This, combined with the ability to transfer lateral roots into a 


\section{Temperature dependency of Agrobacterium rhizogenes-mediated root}

transformation

rhizobium hosting organ raises the intriguing question why most plants have not yet evolved a rhizobium nodule symbiosis. As Parasponia could provide the answer a Chinese-Dutch consortium has started the sequencing of the $P$. andersonii genome. However, to fully exploit this sequence information it will be essential to have an efficient transformation procedure available.
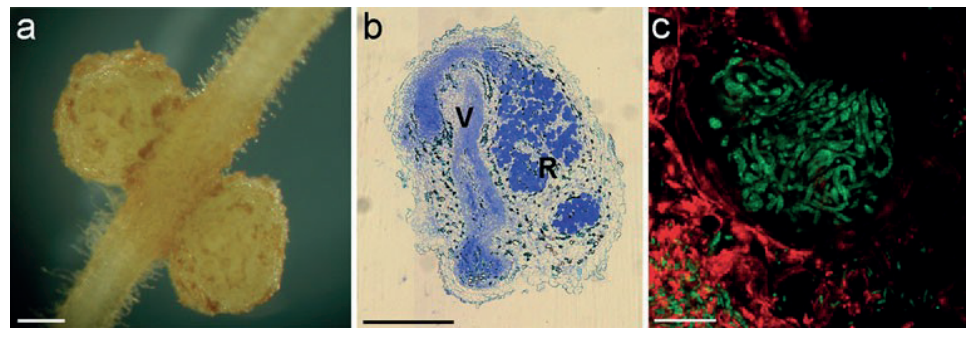

Fig. 1 Parasponia root nodules. a Nodules on P. andersonii roots induced by Sinorhizobium sp. NGR234. Bar 1 $\mathrm{mm}$. b Structure of a $P$. andersonii nodule with central vascular bundle (V) and peripheral lobes with rhizobium infected cells (R). Bar $1 \mathrm{~mm}$. c Confocal microscope image of an infected $P$. andersonii nodule cell filled with fixation threads. Rhizobia inside these fixation threads express the fluorescent marker gene GFP. Cells are counterstained with FM-64. Bar $1 \mathrm{~mm}$.

Genetic transformation mediated by A. tumefaciens or A. rhizogenes has been developed for many plant species. In legumes especially co-transformation with A. rhizogenes has been used. In this system the root inducing locus ( $r o l$ ) genes of $A$. rhizogenes are transferred to the host and induce the formation of so-called hairy roots, even though the phenotypic appearance of these roots are often indistinguishable from untransformed roots (White et al., 1985). The gene of interest can be co-transferred with the rol genes. A. rhizogenes mediated transformation leads to composite plants with a non-transgenic shoot carrying several transgenic roots that are the result of independent transformation events. For a number of legume species this method has been optimized, which resulted in rapid and effective protocols to generate composite plants (Boisson-Dernier et al., 2001; Limpens et al., 2004; Colpaert et al., 2008; Estrada-Navarrete et $a l ., 2006)$. Since a composite plant may contain co-transformed as well as non-co-transformed roots, generally a fluorescent protein (e.g. DsRed or GFP) is used as non-destructive selectable 


\section{Chapter 2}

marker. Taken together, these protocols provide a solid platform for studies on legume root biology and root-microbe interactions, such as rhizobium and endomycorrhizal symbioses. Here, we aim to establish a similar platform for $P$. andersonii and its non-nodulation sister species Trema tomentosa.

\section{Results and discussion}

\section{Agrobacterium rhizogenes-mediated root transformation of Parasponia andersonii is temperature dependent}

Parasponia andersonii is a tropical woody plant, and the optimal temperature for growth of seedlings or micro-propagated shoots is $28^{\circ} \mathrm{C}$ (Davey et al., 1993; Webster et al., 1995). Shoot cuttings can be efficiently rooted at this temperature. Therefore we tested whether $A$. rhizogenes would induce the formation of transgenic roots at $28^{\circ} \mathrm{C}$. For this experiment we used micropropagated plantlets that had already formed roots and the A. rhizogenes strain MSU440 that harbored a control binary vector containing DsRed1 as selectable marker. Plantlets were infected with $A$. rhizogenes after removing the root system and were inoculated at the wound surfaces. Plantlets and A. rhizogenes were co-cultivated for 5 days on EKM medium and subsequently transferred to emergence medium that is rich in nutrients and contains cefotaxime to kill $A$. rhizogenes. These plants formed about 3 new roots within a 3 week time period. However, the number of transgenic roots expressing DsRedl was very low. We analyzed 222 plants and only 8 contained one or more transgenic roots based on red fluorescence (Fig. 2a, b; Table 1). As the transformation efficiency ( $\%$ of composite plants with a transgenic root) was only $3.6 \%$, this method was not really suitable for research and we aimed to optimize it.

Boisson-Dernier et al. (2001) had previously shown that a mild temperature during cocultivation is beneficial for A. rhizogenes-mediated transformation of M. truncatula. Therefore, we tested whether a lower co-cultivation temperature could improve the efficiency of $A$. rhizogenes mediated transformation of $P$. andersonii. Again, we used micropropagated plantlets that had already formed roots. 
Temperature dependency of Agrobacterium rhizogenes-mediated root

transformation
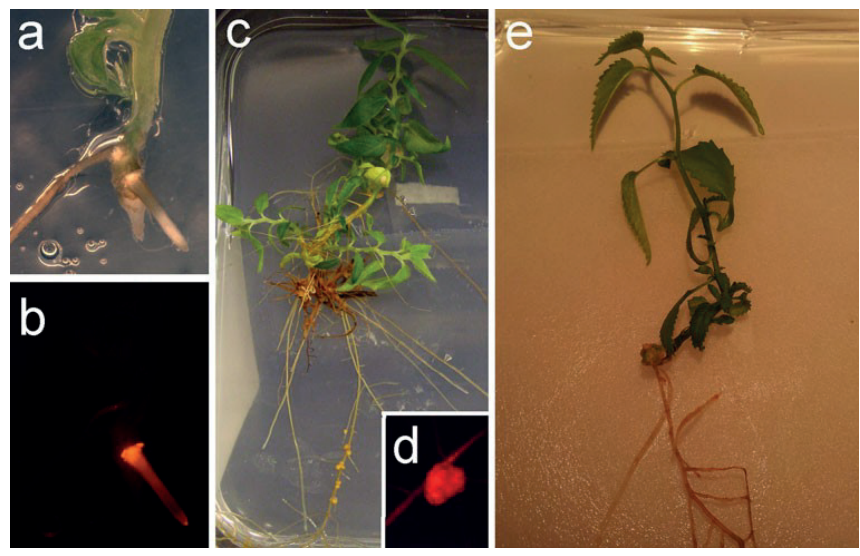

Fig.2 Micropropagated Parasponia andersonii and Trema tomentosa plantlets with transgenic roots. a, b $P$. andersonii plantlet carrying a transgenic root, which can be distinguished based on DSRED1 expression causing red fluorescence (b). c, d P. andersonii plantlet carrying a transgenic root that is nodulated with Sinorhizobium NGR234. Transgenic root nodules can distinguished based on red fluorescence (d). e Rooted T. tomentosa plantlet. 


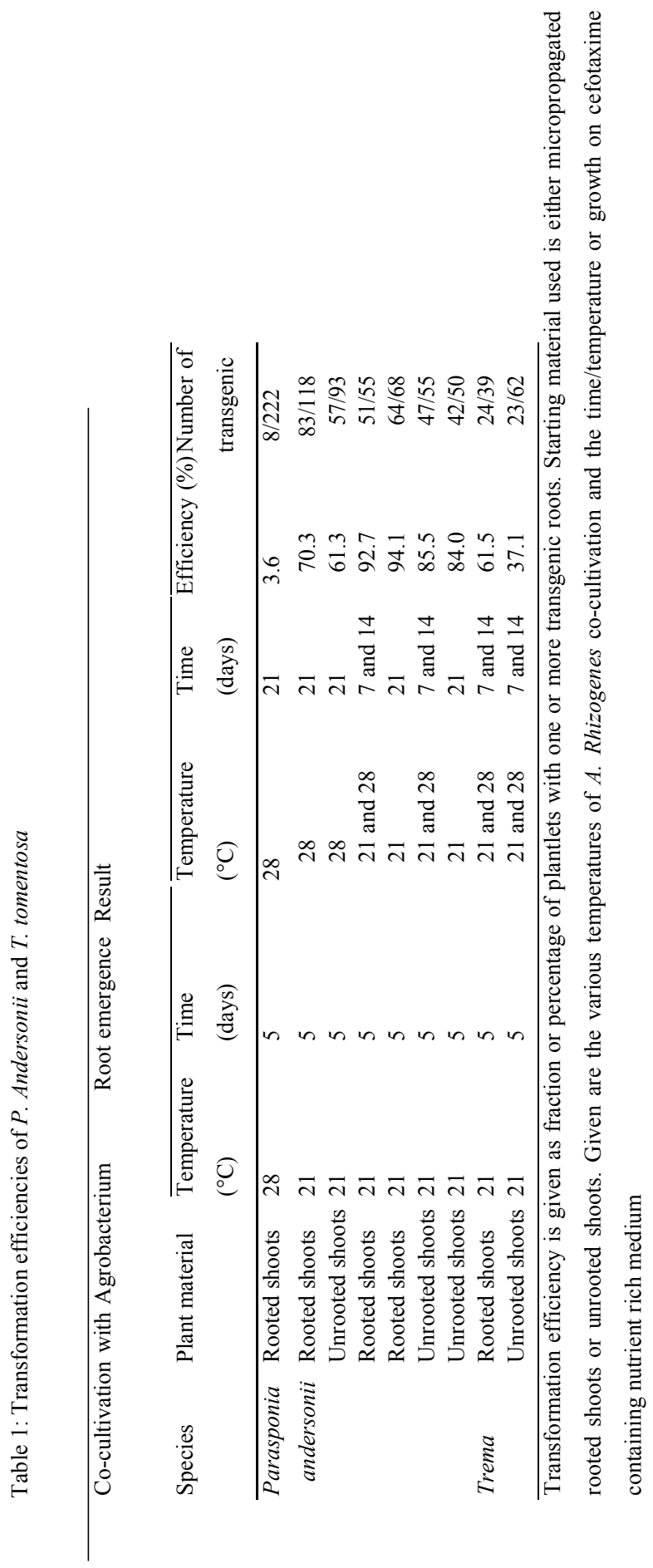


P.andersonii was co-cultivated with A. rhizogenes at $21{ }^{\circ} \mathrm{C}$ on EKM medium. Five days after inoculation plants were transferred to emergence medium (including cefotaxime) and placed back at $28{ }^{\circ} \mathrm{C}$, which is the optimal temperature for growth of $P$. andersonii. Three weeks after transfer, the transformation efficiency of micropropagated plantlets was $70.3 \%$ (Table 1). Based on these findings we conclude that co-cultivation at lower temperature significantly increases the transformation efficiency of $P$. andersonii.

Next, we tested whether prolonged growth at $21^{\circ} \mathrm{C}$ can further improve the transformation efficiency. To do so, we performed two types of experiments. After co-cultivation at $21^{\circ} \mathrm{C}$ for 5 days, plants were transferred to emergence medium and kept for 1 or 3 additional weeks at 21 ${ }^{\circ} \mathrm{C}$. Both growth conditions improved transformation, resulting in almost $100 \%$ efficiency (Fig. 2; Table 1). So, extending the period that plants are grown at $21^{\circ} \mathrm{C}$ is the key to further increase the transformation efficiency. However, transgenic roots grew very slow at $21^{\circ} \mathrm{C}$ and large calli developed at the base on the root system when grown at this temperature for 3 weeks. Therefore, we conclude that the best way to obtain proper transgenic roots in an efficient manner is by co-cultivation at $21^{\circ} \mathrm{C}$ for 5 days and an additional week at $21{ }^{\circ} \mathrm{C}$ when grown on cefotaxime containing nutrient rich emergence medium, prior transfer to $28^{\circ} \mathrm{C}$. These transgenic roots could also be nodulated when grown on plates by Sinorhizobium sp. NGR234 (Fig. 2c, d).

\section{Transformation of unrooted Parasponia andersonii shoots}

In the above described method we used rooted plantlets as source for transformation. These plantlets were obtained upon in vitro micropropagation as described by Davey et al. (1993) with a few modifications. As $P$. andersonii is a tree, we decided to use woody plant medium (WPM, (Lloyd, 1980) instead of MS medium. Generated shoots were subsequently rooted on auxin containing rooting medium; a procedure that takes 2 weeks. We raised the question whether unrooted shoots could be used directly for A. rhizogenes -mediated root transformation. This would be attractive as shoots can be obtained in high numbers with low labour input. The initial protocol for rooted plantlets $\left(5\right.$ days at $21^{\circ} \mathrm{C}, 3$ weeks at $28^{\circ} \mathrm{C}$ ) resulted in a transformation efficiency of $61.3 \%$ (Table 1). Either method of prolonged growth at $21^{\circ} \mathrm{C}$ resulted in a higher transformation efficiency of $85 \%$ (Table 1). Taken 


\section{Chapter 2}

together, we conclude that also unrooted shoots can be used to generate composite plants carrying transgenic roots with efficiency comparable to rooted plantlets. By doing so, the time period of experimentation is shortened by about 2 weeks.

\section{Micropropagation and root transformation of Trema tomentosa}

To enable comparative studies with Parasponia we aimed to develop a similar root transformation system for the closely related non-nodulating sister species, namely $T$. tomentosa. We set up an in vitro micropropagation system similar as used for P. andersonii. The in vitro micropropagation method as applied for $P$. andersonii could be adopted with marginal changes for $T$. tomentosa (see "Materials and methods"). We used the optimized protocol for A. rhizogenes-mediated transformation of $P$. andersonii and used either rooted or unrooted T. tomentosa shoots as starting material. The efficiency of rooted T.tomentosa plantlets was $61.5 \%$ (Fig. 2e; Table 1). But when unrooted shoots are used an efficiency of only $37.1 \%$ was obtained (Table 1). Most of untransformed shoots had big calli at the wound site of $A$. rhizogenes infection. This may decrease the transformation efficiency. Still the majority of the rooted plantlets could be transformed and therefore we concluded that rooted plantlets are a better starting material for A. rhizogenes-mediated transformation of $T$. tomentosa.

\section{Conclusion}

We developed a relatively fast and highly efficient method for $A$. rhizogenes-mediated roots transformation for Parasponia and Trema species. We show that in case of $P$. andersonii and $T$. tomentosa transformation with $A$. rhizogenes MSU440 at $21^{\circ} \mathrm{C}$ is most efficient despite the fact that the optimal growth temperature of these tropical species is $28^{\circ} \mathrm{C}$. This is in line with previous $A$. rhizogenes- based root transformation studies on $M$. truncatula and supports the conclusion that Agrobacterium mediated DNA transfer is temperature dependent (Dillen et al., 1997; Kondo et al., 2000; Boisson-Dernier et al., 2001; Salas et al., 2001). The efficient transformation protocols will be an important tool for functional analysis of Parasponia and Trema genes by reverse genetics. This technology will facilitate the use of the Parasponia genome sequence that will become available in the near future and so it will be instrumental in unraveling the evolutionary mechanism by which Parasponia obtained the ability to 
establish a $\mathrm{N}_{2}$ fixing symbiosis with Rhizobium.

\section{Materials and methods}

\section{In vitro micro-propagation of $P$. andersonii and $T$. Tomentosa}

Parasponia andersonii and Trema tomentosa axillary buds were surface sterilized in $4 \%$ hypochlorite (commercial bleach) for $7 \mathrm{~min}$ and subsequently washed 6 times by autoclaved water. Sterilized $P$. andersonii axillary buds were placed on propagation medium in $\varnothing 10 \mathrm{~cm}$ round petri dishes $(\mathrm{pH} 5.8,20 \mathrm{~g} / \mathrm{L}$ sucrose, $2.4 \mathrm{~g} / \mathrm{L}$ McCown Woody Plant Medium (WPM) including vitamins (Duchefa Biochemie, Haarlem, The Netherlands; WPM) (Lloyd, 1980) 1.0 $\mathrm{mg} / \mathrm{L}$ 6-Benzylaminopurine, $0.1 \mathrm{mg} / \mathrm{L}$ Indole-3-butyric acid, $0.8 \%$ Daichin agar (Brunschwig Chemie, Amstedam, The Netherlands)). Sterile T. tomentosa axillary buds were placed on

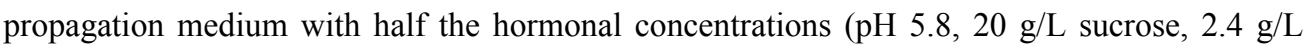
WPM, $0.5 \mathrm{mg} / \mathrm{L} \quad$ 6-Benzylaminopurine, $0.05 \mathrm{mg} / \mathrm{L}$ Indole-3-butyric acid, $0.8 \%$ Daichin agar). Both were kept in an Elbanton growth cabinet at $28^{\circ} \mathrm{C}$ with a $16 / 8 \mathrm{~h}$ day/night regime. Within 4 weeks shoots emerged from the axillary buds calli. Shoots of $1.0 \mathrm{~cm}$ in length were cut and transferred to rooting medium (pH5.8, $20 \mathrm{~g} / \mathrm{L}$ sucrose, $2.4 \mathrm{~g} / \mathrm{L} \mathrm{WPM}, 1.0 \mathrm{mg} / \mathrm{L}$ indole-3-butyric acid, $0.1 \mathrm{mg} / \mathrm{L}$ 1-naphthaleneacetic acid) in round $\varnothing 10 \mathrm{~cm}$ petri dished in-between two halfround $\varnothing 8.5 \mathrm{~cm}$ filter papers (Machery-Nagel, Düren, Germany). These plates were covered by aluminum foil (darkness induced roots) and kept in the same growth cabinet at $28^{\circ} \mathrm{C}$ for 2 weeks. Rooted shoots were kept on hormone free medium $(\mathrm{pH} \mathrm{5.8,} 20 \mathrm{~g} / \mathrm{L}$ sucrose, $2.4 \mathrm{~g} / \mathrm{L}$ WPM, and $0.8 \%$ Daichin agar).

\section{Bacterial strains}

Agrobacterium rhizogenes strain MSU440 carrying the empty binary vector pRed Root that contains DsRed1 as selectable marker was used for the transformations (Lim- pens et al. 2004). The binary vector is available upon request from our laboratory.

Agrobacterium rhizogenes-mediated transformation micropropagated $P$. andersonii or $T$. tomentosa shoots (over $1.0 \mathrm{~cm}$ in length) or rooted plants were used for $A$ rhizogenes 


\section{Chapter 2}

transformation. Roots or the basipetal tip of a shoot stem were removed from the plantlets/shoots with a razor blade and plants were subsequently placed on top of a $\varnothing 8.5 \mathrm{~cm}$ half-round filter (Machery-Nagel, Düren, Germany) on EKM medium $\varnothing 10 \mathrm{~cm}$ petri dishes $(\mathrm{pH} \quad 6.6, \quad 0.25 \mathrm{~g} / \mathrm{L} \quad \mathrm{MgSO} 4.7 \mathrm{H} 2 \mathrm{O}, 0.12 \mathrm{~g} / \mathrm{L} \quad \mathrm{KH} 2 \mathrm{PO} 4,0.36 \mathrm{~g} / \mathrm{L} \quad \mathrm{K} 2 \mathrm{HPO} 4,0.25 \mathrm{~g} / \mathrm{L}$ CaSO4.2H2O, $0.10 \mathrm{~g} / \mathrm{L} \quad \mathrm{Na} 2 \mathrm{SO} 4,0.03 \mathrm{~g} / \mathrm{L} \quad \mathrm{NH} 4 \mathrm{NO} 3,0.005 \mathrm{~g} / \mathrm{L}$ Fe-citrate, $1.0 \mathrm{mg} / \mathrm{L}$ MnSO4, 0.25 mg/L ZnSO4.7H2O, 0.25 mg/L CuSO4.5H2O, 0.25 mg/L H3BO3, 0.005 mg/L Na2MoO4.2H2O, 0.8 \% Daichin agar (Bruns- chwig Chemie, Amstedam, The Netherlands)). Plate grown A. rhizogenes strain MSU440 carrying pRedRoot was applied on the wounded surface. The most basipetal shoot portion was carefully punctured 2-3 times with a sterile needle (0.45 micron) that was first dipped in A. rhizogenes. Plates with plantlets were placed vertically in the growth cabinet. First, the wounded shoots were co-cultivated with $A$. rhizogenes on EKM (in Elbanton growth cabinet at either 21 or $28^{\circ} \mathrm{C}$ with $16 / 8 \mathrm{~h}$ light/dark cycle), and subsequently transferred to cefotaxime $(300 \mathrm{mg} / \mathrm{ml})$ containing nutrient rich emergence medium (EM) (3 mM MES pH 5.8 containing $2.5 \mathrm{~g} / \mathrm{L}$ KNO3, $0.4 \mathrm{~g} / \mathrm{L}$ $\mathrm{MgSO} 4 \cdot 7 \mathrm{H} 2 \mathrm{O}, 0.3 \mathrm{~g} / \mathrm{L}$ NH $4 \mathrm{H} 2 \mathrm{PO} 4,0.2 \mathrm{~g} / \mathrm{L}$ CaCl $2 \cdot 2 \mathrm{H} 2 \mathrm{O}, 10 \mathrm{mg} / \mathrm{L} \mathrm{MnSO} 4 \cdot 4 \mathrm{H} 2 \mathrm{O}, 5 \mathrm{mg} / \mathrm{L}$ H3BO3, $1 \mathrm{mg} / \mathrm{L} \quad \mathrm{ZnSO} 4 \cdot 7 \mathrm{H} 2 \mathrm{O}, 1 \mathrm{mg} / \mathrm{L} \quad \mathrm{KI}, \quad 0.2 \mathrm{mg} / \mathrm{L} \quad \mathrm{CuSO} 4 \cdot 5 \mathrm{H} 2 \mathrm{O}, \quad 0.1 \mathrm{mg} / \mathrm{L}$ $\mathrm{NaMoO} 4 \cdot 2 \mathrm{H} 2 \mathrm{O}, 0.1 \mathrm{mg} / \mathrm{L} \mathrm{CoCl} 2 \cdot 6 \mathrm{H} 2 \mathrm{O}, 15 \mathrm{mg} / \mathrm{L}$ FeSO $4 \cdot 7 \mathrm{H} 2 \mathrm{O}, 20 \mathrm{mg} / \mathrm{L} \mathrm{Na} 2 \mathrm{EDTA}, 100$ $\mathrm{mg} / \mathrm{L}$ myoinositol, $5 \mathrm{mg} / \mathrm{L}$ nicotinic acid, $10 \mathrm{mg} / \mathrm{L}$ pyridoxine $\mathrm{HCl}, 10 \mathrm{mg} / \mathrm{L}$ thiamine $\mathrm{HCl}, 2$ $\mathrm{mg} / \mathrm{L}$ glycine, $10 \mathrm{~g} / \mathrm{L}$ sucrose, $0.9 \%$ Daichin agar containing $300 \mathrm{mg} / \mathrm{ml}$ Cefotaxime Sodium (Duchefa Biochemie, Haarlem, The Netherlands)) (Elbanton growth cabinet at either 21 or 28 ${ }^{\circ} \mathrm{C}$ with 16/8 h light/dark cycle). On EM medium shoots were kept in-between two half-round filter papers; mind especially that both filters are in contact with the wounded surface. Petri dishes were closed with parafilm, but a small opening was left to enable aeration.

\section{Detection of transgenic roots}

Transgenic roots were selected based on red fluorescence, using a Leica MZIII fluorescence stereomicroscope (filter settings; excitation 565/30 and emission 620/60). The number of transformed roots was determined based on DsRed1 fluorescence.

\section{Nodulation of $P$. andersonii composite plants}

Parasponia andersonii plantlets with transformed roots were transferred to EKM supplemented 
Temperature dependency of Agrobacterium rhizogenes-mediated root

transformation

with 2 lM Aminoethoxyvinylglycine (AVG) in $120 \times 9 \times 120 \mathrm{~cm}$ square petri dishes of which the

lower half was streaked with plate grown Sinorhizobium sp. NGR234. Plates were kept vertically in an Elbanton growth cabinet at $28^{\circ} \mathrm{C}$ with $16 / 8 \mathrm{~h}$ light/dark cycle. Nodules appeared within 4-6 weeks.

\section{Acknowledgments}

This work was supported by the Dutch Science Foundation (NWO) (VIDI 864.06.007 to R.G.) and a visitor's fellowship of the Dutch Graduate School of Experimental Plant Sciences to Q.C. 


\section{Chapter 2}

\section{References}

Akkermans, A., et al. (1978). "N2-fixing root nodules in Ulmaceae: Parasponia or (and) Trema spp.?" Plant and Soil 49(3): 711-715.

Beatty, P. H. and A. G. Good (2011). "Future prospects for cereals that fix nitrogen." Science 333(6041): $416-417$.

Becking, J. (1992). The Rhizobium symbiosis of the nonlegume Parasponia, Routledge: Chapman and Hall, NY, USA: $497-559$.

Boisson-Dernier, A., et al. (2001). "Agrobacterium rhizogenes-transformed roots of Medicago truncatula for the study of nitrogen-fixing and endomycorrhizal symbiotic associations." Molecular Plant-Microbe Interactions 14(6): 695-700.

Burrill, T. J. and R. Hansen (1917). "Is symbiosis possible between legume bacteria and non-legume plants?" Bulletin (University of Illinois (Urbana-Champaign campus). Agricultural Experiment Station); no. 202.

Colpaert, N., et al. (2008). "Composite Phaseolus vulgaris plants with transgenic roots as research tool." $\underline{\text { African }}$ Journal of Biotechnology 7(4).

Davey, M., et al. (1993). "Effective nodulation of micro-propagated shoots of the non-legume Parasponia andersonii by Bradyrhizobium." Journal of Experimental Botany 44(5): 863-867.

Dawson, C. J. and J. Hilton (2011). "Fertiliser availability in a resource-limited world: Production and recycling of nitrogen and phosphorus." Food Policy 36: S14-S22.

den Camp, R. O., et al. (2011). "LysM-type mycorrhizal receptor recruited for rhizobium symbiosis in nonlegume Parasponia." Science 331(6019): 909-912.

Dillen, W., et al. (1997). "The effect of temperature on Agrobacterium tumefaciens-mediated gene transfer to plants." The Plant Journal 12(6): 1459-1463.

Estrada-Navarrete, G., et al. (2006). "Agrobacterium rhizogenes transformation of the Phaseolus spp.: a tool for functional genomics." Molecular Plant-Microbe Interactions 19(12): 1385-1393.

Guo, J., et al. (2010). "A single origin and moderate bottleneck during domestication of soybean (Glycine max): implications from microsatellites and nucleotide sequences." Annals of Botany 106(3): 505-514.

II, A. (2003). "An update of the Angiosperm Phylogeny Group classification for the orders and families of flowering plants: APG II." Botanical Journal of the Linnean Society 141(4): 399-436.

Kondo, T., et al. (2000). "Transformation and regeneration of garlic (Allium sativum L.) by Agrobacteriummediated gene transfer." Plant Cell Reports 19(10): 989-993.

Lee, G.-A., et al. (2007). "Plants and people from the Early Neolithic to Shang periods in North China." Proceedings of the National Academy of Sciences 104(3): 1087-1092.

Limpens, E., et al. (2004). "RNA interference in Agrobacterium rhizogenes-transformed roots of Arabidopsis and Medicago truncatula." Journal of Experimental Botany 55(399): 983-992.

Lloyd, G. (1980). Commercially-feasible micropropagation of mountain laurel, Kalmia latifolia, by use of shoot-tip culture. Int. Plant. Prop. Soc. 
Salas, M., et al. (2001). "Temperature influence on stable T-DNA integration in plant cells." Plant Cell Reports 20(8): 701-705.

Streng, A., et al. (2011). "Evolutionary origin of rhizobium Nod factor signaling." Plant Signaling \& Behavior 6(10): $1510-1514$.

Sytsma, K. J., et al. (2002). "Urticalean rosids: circumscription, rosid ancestry, and phylogenetics based on rbcL, trnL-F, and ndhF sequences." American Journal of Botany 89(9): 1531-1546.

Trinick, M. (1973). "Symbiosis between Rhizobium and the non-legume, Trema aspera." Nature 244: 459-460.

Trinick, M. (1979). "Structure of nitrogen-fixing nodules formed by Rhizobium on roots of Parasponia andersonii Planch." Canadian Journal of Microbiology 25(5): 565-578.

Wang, H., et al. (2009). "Rosid radiation and the rapid rise of angiosperm-dominated forests." Proceedings of the National Academy of Sciences 106(10): 3853-3858.

Webster, G., et al. (1995). "The nodulation of micro-propagated plants of Parasponia andersonii by tropical legume rhizobia." Journal of Experimental Botany 46(9): 1131-1137.

White, F., et al. (1985). "Molecular and genetic analysis of the transferred DNA regions of the root-inducing plasmid of Agrobacterium rhizogenes." Journal of bacteriology 164(1): 33-44.

Yesson, C., et al. (2004). "Phylogenetic framework for Trema (Celtidaceae)." Plant Systematics and Evolution 248(1-4): 85-109.

Young, N. D., et al. (2011). "The Medicago genome provides insight into the evolution of rhizobial symbioses." Nature 480(7378): 520-524. 


\section{Chapter 3}

Agrobacterium tumefaciens-mediated Stable Transformation of Trema tomentosa

Maryam Seifi Kalhor, Marijke Hartog, Tingting Xiao, Ton Bisseling and René Geurts 


\section{Chapter 3}

\section{Abstract}

Tropical tree species of Trema genus are the closest relatives of Parasponia tree plants; the only known non-legume lineage of plant species that are capable of establishing a nitrogen fixing nodule symbiosis with rhizobium. To identify genetic elements that underlie the rhizobium symbiosis, comparative studies of Parasponia sp. and Trema sp. are markedly informative. Generally, Agrobacterium rhizogenes-mediated root transformation is used to conduct reverse genetic experiments in a root symbiosis context. However, such transient methods are prone for variation, as every transgenic root is the result of a de novo transformation event. Here, we present a protocol for Agrobacterium tumefaciens-mediated stable transformation of Trema tomentosa. To do so, we used a binary vector containing the auxin responsive reporter DR5::GUS and two selectable marker genes; NPTII encoding kanamycin resistance and the red fluorescent protein encoding gene DsRED1. By using culture conditions optimized for $A$. tumefaciens co-cultivation, callus formation and regeneration of plantlets, a transformation efficiency of $15 \%$ was obtained. In vitro propagated T0 plants display a DR5::GUS expression in leaves that is identical to observed in primary T0 plantlets. Taken together, the presented transformation method for T. tomentosa is efficient, and results in transgenic plantlets within a timeframe of only 6 months.

Keywords: Trema tomentosa, Co-cultivation, Agrobacterium tumefaciens, DR5::GUS. 


\section{Introduction}

Nitrogen fixing rhizobium symbiosis is a well-known character of leguminous plants (Fabaceae). Beside legume plants, a comparable symbiosis also occurs on five tropical tree species of the Parasponia genus (Cannabaceae). In both legumes as well as Parasponia spp., the interaction with rhizobium results in formation of novel root organs; so-called root nodules. Rhizobium is hosted in the nodules cells, where rhizobium bacteria can find the proper conditions to convert atmospheric nitrogen $\left(\mathrm{N}_{2}\right)$ into ammonia $\left(\mathrm{NH}_{4}{ }^{+}\right)$. The produced ammonia can be utilized by the plant. Parasponia genus and Fabaceae family had a last common ancestor that lived about 100 million years ago (Wang et al., 2009), which makes it most probable that both lineages evolved rhizobium symbiosis independently. Several lines of evidence indicate that genetic constraints have guided evolution of symbiosis in both lineages. Root nodule formation in legumes and Parasponia sp. can occur with the same rhizobium bacteria, requires very similar bacterial lipochitooligosaccharide (LCO) signal molecules, and is controlled by a conserved symbiotic signaling network (den Camp et al., 2011). Therefore, the Parasponia research model -alongside with legume model plants like Lotus japonicus and Medicago truncatula-provides a novel tool to obtain insights in the genetics underlying the nitrogen fixing endosymbiosis with rhizobium.

Parasponia is highly promiscuous as it will allow most LCO-secreting rhizobia to enter its roots by a mechanism known as crack entry (Op den Camp et al., 2012) These rhizobium bacteria remain in the apoplast, and only will infect intracellularly when reaching nodule cells. Once inside a cell, the bacteria remain within infection thread-like structures known as fixation threads. Interestingly, it was noted that Parasponia nodules are less effective in nitrogen fixation when compared to nodules of legumes formed by the same bacteria (Behm et al., 2014). This may be explained by the limited colonization efficiency of infection threads, when compared to most legume nodules that host rhizobia as organelle-like structures (known as symbiosomes). Rhizobia in fixation threads may also have less efficient exchange of nutrients when compared to legume symbiosomes. Further, Parasponia nodules contain only a single -centrally positionedvascular bundle, whereas legume nodules have several peripheral vascular bundles, which may limit its transport capacity. Taken-together, it suggests that Parasponia root nodules are more primitive when compared to nodules formed on legume plants (Behm et al., 2014). 


\section{Chapter 3}

Rhizobium LCOs are perceived by specific LysM-type receptor kinases. Studies in legumes and Parasponia indicated that -at least for one receptor- the same gene has been cooped to commit such symbiotic function (den Camp et al., 2011)These receptors activate a highly conserved symbiotic signaling network that stretches from an additional LRR-type receptor down to transcriptional regulators. This symbiotic signaling pathway is highly conserved, and present in most plant species as it is essential also for endomyocrrhizal symbiosis (Roberts et al., 2013). The importance of this symbiotic signaling network for root nodule formation is underlined by ectopic expression studies and the use of dominant alleles that trigger spontaneous root nodule formation in legumes and Parasponia (den Camp et al., 2011). However, it remains elusive whether adaptations have occurred in any of symbiotic genes that is essential for the nodulation trait in legumes and/or Parasponia.

Despite of extensive research especially in model legumes, the essential genetic adaptations that allow plants to form nitrogen fixing root nodules have not yet been characterized. It may be because of the exclusive focus on symbiotic plants (e.g. legumes or Parapsonia) to dissect the genetics underlying rhizobium symbiosis. Rarely comparative studies with close relatives that are unable to form nitrogen fixing root nodules are committed.

We advocate the adaptation of Trema tomentosa as comparative plant system for Parasponia. Parasponia and Trema are closely related sister genera and recent molecular phylogenetic studies even suggest that the Parasponia-Trema lineage is paraphyletic (Akkermans et al., 1978; Yang et al., 2013). This suggests that Parasponia -including its symbiotic trait- is relatively young and evolved from an ancestral Trema sp. In contrast to Parasponia that is native to volcanic regions in the Malay Archipelago, Trema sp. are pantropical. In total 15 Trema species are recognized, however the taxonomy of this genus has only partially been resolved. We selected T. tomentosa, a species native to northern Australia, as workable model. T. tomentosa trees flower within 6-9 months under greenhouse conditions and can be propagated vegetatively in vitro. Additionally, it was shown that $T$. tomentosa harbouring transgenic roots can be obtained using Agrobacterium rhizogenes-mediated transformation (Cao et al., 2012). By using this method to introduce the Cameleon calcium sensor, we could confirm that $T$. tomentosa doesn't show symbiotic responses in form of regular $\mathrm{Ca}^{2+}$ oscillation upon application of rhizobium LCOs, whereas this response is triggered in Parasponia (Granqvist et al., 2015). 
Although commonly used in legume and Parasponia research, A. rhizogenes mediated root transformation has several disadvantages. First, the generated roots represent primary transformed tissue that cannot be characterized in terms of position and number of T-DNAs integrated. Such roots therefore may vary in expression level of the transgene. By default, compound plants carrying transgenic roots can only be used transiently, in other words, experiments have to be conducted with roots of independent transformation events. An additional flaw of $A$. rhizogenes is the transfer of the root inducing locus (rol) genes. These genes are essential to trigger root formation in the plant, but they interfer with the plant root's hormone balance. As a result, A. rhizogenes transformed roots may differ in phenotype, and therefore such roots are not suitable for studying plant hormones. In the case of Parasponia and Trema we showed that transformation with A. rhizogenes had also technical difficulties for transformation efficiencies (Cao et al., 2012) and overgrowth of tissue by this pathogenic bacterium. This let us to decide to investigate whether Parasponia and/or Trema can be transformed with $A$. tumefaciens to generate stable transgenic lines.

Here we present a protocol for A. tumefaciens mediated transformation of T. tomentosa. By using a binary vector containing the auxin responsive reporter DR5::GUS and two selectable markers; NPTII encoding kanamycin resistance and the RED FLUORSCENT PROTEIN encoding gene DSRED. We demonstrate that transgenic T. tomentosa plantlets could be generated within 6 month. These plantlets can be vegetatively propagated without losing transgene expression.

\section{Results}

\section{Agrobacterium tumefaciens mediated transformation of Trema tomentosa}

For T. tomentosa an in vitro propagation protocol has been established (Cao et al., 2012). In this protocol shoot axillary buds are used as starting material. We tested whether we can use this material as starting material for $A$. tumefaciens mediated transformation. To do so, we made use of the A. tumefaciens strain AGL1 harbouring the binary plasmid, pT14DR5. This plasmid contains the auxin responsive synthetic promoter DR5 driving GUS. Additionally, it contains three selectable markers; namely NPTII that convers resistance to kanamycin, DsREDI driven by the constitutive $A t U B Q 10$ promoter of Arabidopsis thaliana and spectinomycin adenyltransferase gene $(A A D A)$ for bacteria selection in the backbone (Figure 1). 


\section{Chapter 3}

Axillary buds were either sliced in segments or used as a whole. All explants were immersed in an $A$. tumefaciens culture (O.D.600=0.6) for $30 \mathrm{~min}$ and placed on WPM culture medium supplemented with $1 \mathrm{ml}$ acetosyringone $(20 \mu \mathrm{g} / \mathrm{ml})$ and incubated in the dark for 3 days at $21{ }^{\circ} \mathrm{C}$ for co-cultivation. Next, explants were transferred to regeneration medium containing cefotaxime (300 mg/litr) and kanamycin (50mg/1lite) and transferred to $28^{\circ} \mathrm{C}$ and a $16 / 8 \mathrm{~h}$ day/night regime.

To determine which auxin/cytokinin dose is most effective for inducing regeneration we tested 3 different combinations of 6-benzyladenine (BAP) and indole-3-butyric acid (IBA) (table 1). All three hormone combinations induced callus formation within four weeks after transfer. In all cases, white callus initially developed at the cutting sides of the explants (Figure 2a). However, some calli in both samples (split and whole meristems) turned brownish and obtained a more thick appearance and became overgrown with Agrobacterium (Figure 2b, c). Segmented apical buds showed more efficient callus formation when compared to whole buds (table 1). Also the calli formed on whole buds all turned brown within 4 weeks. These calli stopped growing and could not be investigated further. 


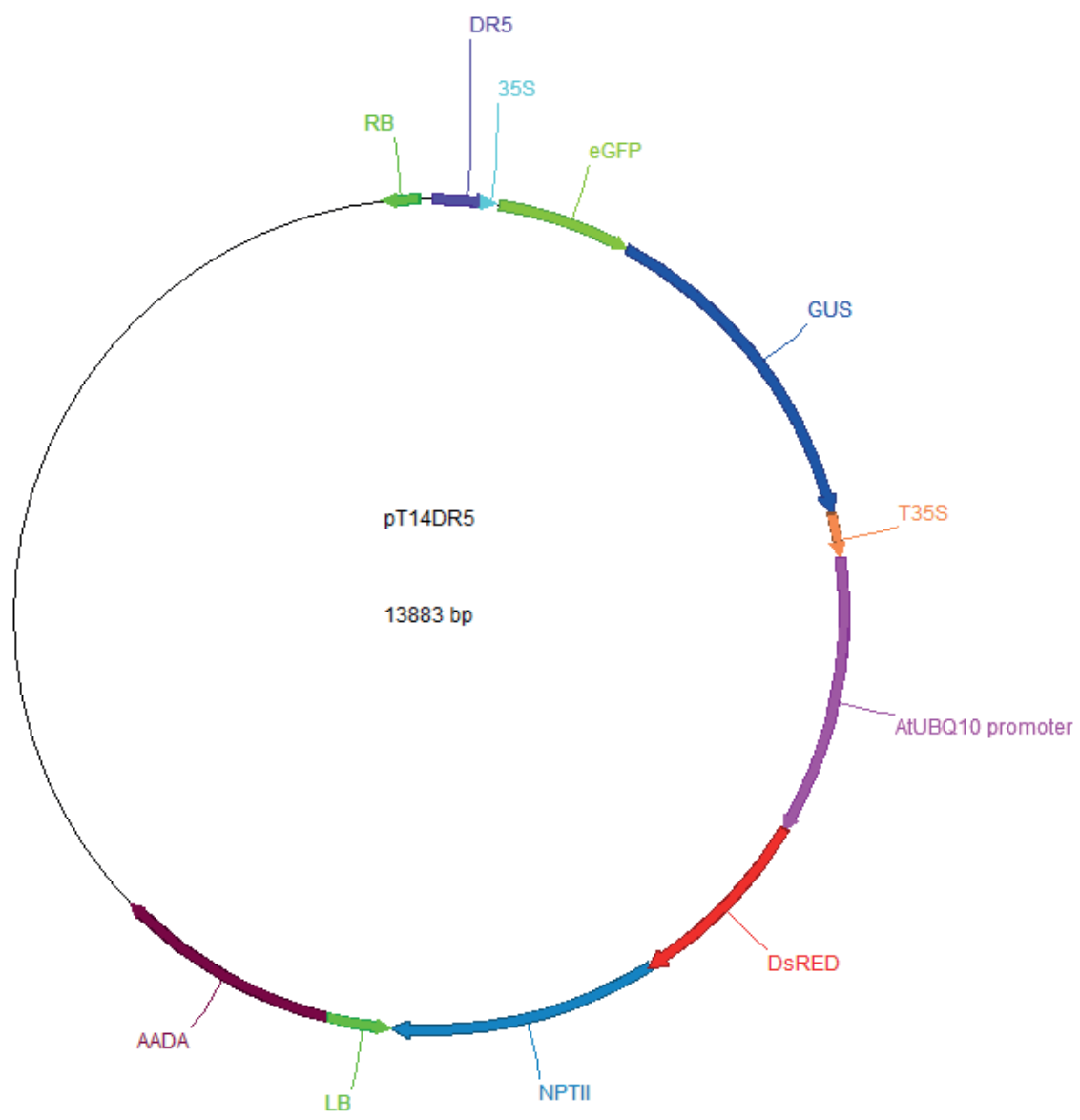

Figure 1. Map of vector pT14DR5containing a DR5::/-glucuronidase (GUS) reporter construct and three selectable markers; DsRED and neomycin phosphotransferase II gene (NPTII) for plant selection in between Left and Right T-DNA borders and spectinomycin adenyltransferase gene (AADA) for bacteria selection in the backbone.

Using segmented axillary buds as explants callus formation was observed on all three media, with comparable efficiencies (Table 1). However, on medium with $1.0 \mathrm{mg} / \mathrm{l} \mathrm{BAP}$ and $0.1 \mathrm{mg} / \mathrm{l}$ IBA (M2) most calli maintained a greenish appearance (Figure 2d). We checked whether green calli were transgenic by monitoring red fluorescence. We noted that only on M3 medium (0.5 


\section{Chapter 3}

mg/l BAP, $0.05 \mathrm{mg} / 1 \mathrm{IBA})$ DsRED expressing calli were found (5 out of 15) (Figure 2e and Table 2).
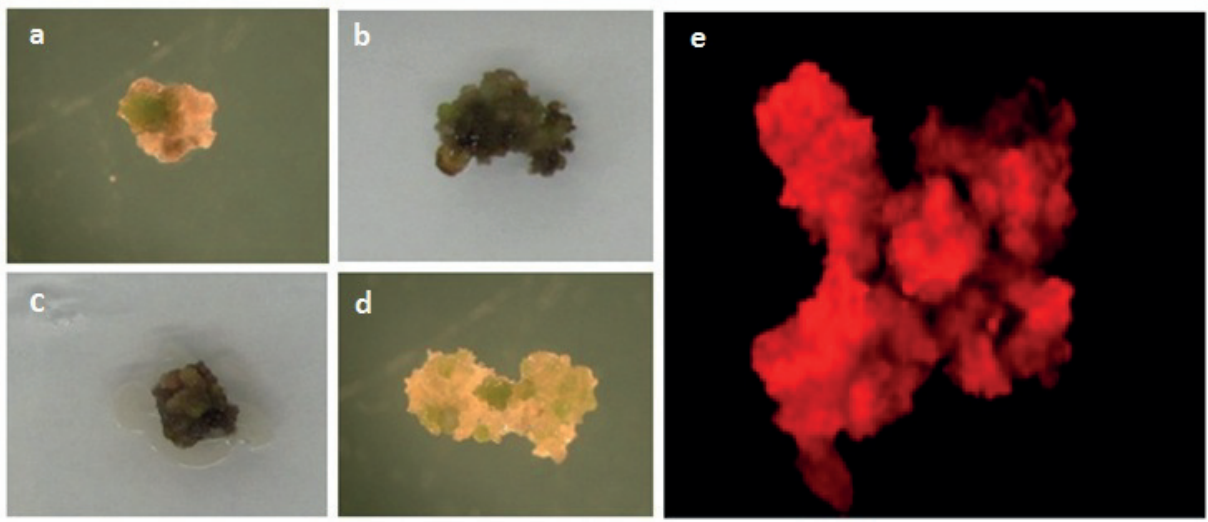

Figure 2. Callus induction from apical meristem culture of Trema tomentosa. a) Callus initiation from apical meristems cultured on WPM medium along with different combination of BAP and IBA b) Thick-brown tissues outgrowth from explants. c) Infected explants before developing callus. d) Green callus emerged after 4 weeks. e) Red florescent transgenic callus.

Table1. Effect of various media on callus induction from apical meristems derived from explants of Trema. tomentosa. Numbers indicate the total number.

\begin{tabular}{|c|c|c|c|c|c|c|c|c|}
\hline \multirow{3}{*}{ Medium } & \multicolumn{8}{|c|}{ Source of the callus } \\
\hline & \multicolumn{4}{|c|}{ Spilt apical meristem } & \multicolumn{4}{|c|}{ Full apical meristem } \\
\hline & callus & $\mathrm{BC}$ & $\mathrm{GC}$ & $\mathrm{TC}$ & callus & $\mathrm{BC}$ & $\mathrm{GC}$ & $\mathrm{TC}$ \\
\hline M1 & 20 & 15 & 5 & 0 & 5 & 5 & 0 & 0 \\
\hline M2 & 40 & 15 & 25 & 0 & 15 & 15 & 0 & 0 \\
\hline M3 & 30 & 15 & 15 & 3 & 0 & 0 & 0 & 0 \\
\hline
\end{tabular}

Subculture media: M1: WPM2.5 mg/1 BAP $+0.25 \mathrm{mg} / 1 \mathrm{IBA} ; \mathrm{M} 2:$ WPM+1.0 mg/1 BAP+0.1mg/l IBA; M3: WPM $+0.5 \mathrm{mg} / 1$ BAP $+0.05 \mathrm{mg} / 1$ IBA. BC: Brown Callus, GC: Green Callus, TC: Transgenic Callus.

Green calli (transgenic (M3 medium) and non-transgenic (M1 \& M2 media)) were split in 4-5 pieces and divided over the same three hormone combinations as used for callus formation (M1, M2 \& M3). Highest shoot regeneration efficiency occurred on M3 medium. On this medium 6 transgenic shoots were derived, originating from 5 primary calli (Figure 3c, Table 2). Also calli cultured on M2 medium formed shoots, although at lower efficiency, whereas on M1 medium no shoots could be regenerated (Figure 3, Table 2). All shoots emerged from calli cultured on M3 medium were confirmed to be transgenic based on DsRED1 mediated red fluorescence (Figure 
$4 a, b)$. These shoots were isolated and transferred to rooting medium to induce root formation as described with Cao et al (Figure 4c) (Cao et al., 2012). All roots were checked for red fluorescence. Four transgenic plants that originated from four different calli were transferred to the greenhouse.
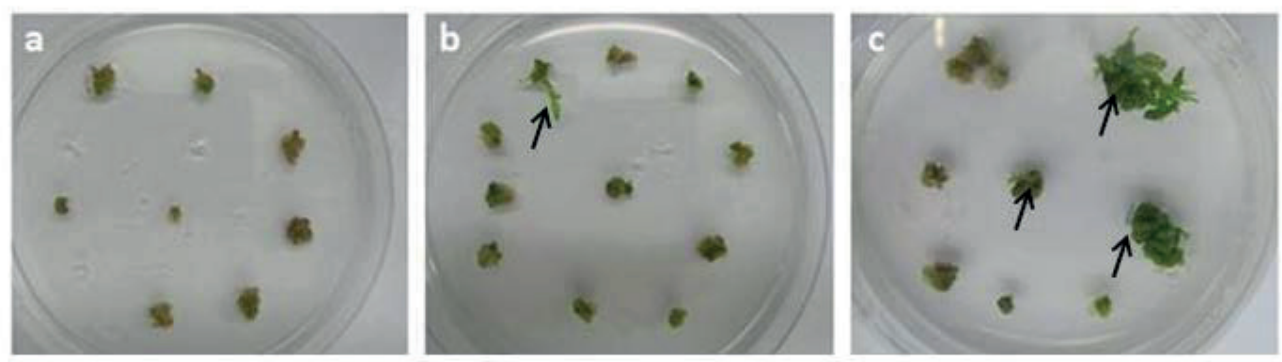

Figure 3. Shoot regeneration from callus in different shoot induction media. a) No shoot regenerate in M1 medium. b) M2 developed two shoots from two calli (in two different plates). c) Five calli on M3 medium form shoots (in three independent plates). The arrows indicate the shoots regenerated from callus.

Table 2. Effect of various media on Shoot and root regeneration from callus in Trema tomentosa.

\begin{tabular}{lccc}
\hline Medium & Number of the callus with shoot & Number of the shoots & number of rooted shoot \\
\hline M1 & 0 & 0 & 0 \\
M2 & $2 / 30$ & 2 & 2 \\
M3 & $5 / 15$ & 6 & 4 \\
\hline
\end{tabular}




\section{Chapter 3}
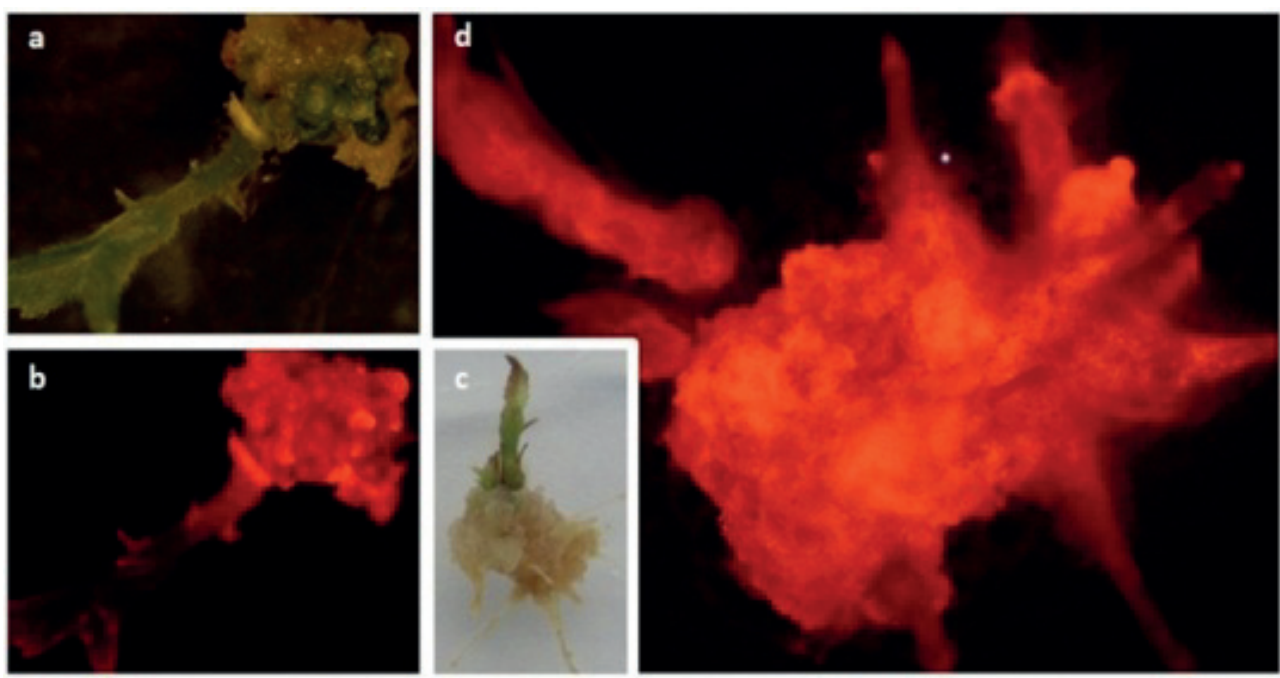

Figure 4. Trema tomentosa shoot and root regeneration from calli that originate from shoot apical meristem. a) Shoot regeneration from apical meristems cultured on WPM+ BAP and IBA for 8 weeks. b) Transgenic shoots can be distinguished based on DsRED1 expression causing red fluorescence. c) Root formation of shoot, after 6 weeks cultivation. d) Red florescence in transgenic roots.

\section{The auxin reporter DR5 is active in meristematic tissue of T. tomentosa}

To check for transgene expression in the four selected transgenic plants we conducted a GUS assay on lateral roots and young leaves. In two out of four plants pattern of blue staining could be observed, with strong signal in the root apical meristem and at the rim of a developing leaf (Figure 5). These M0 plants were maintained in the greenhouse for seed production. DR5::GUS expression was investigated in more detail in transgenic M1 seedlings (Figure 6). Whole mount staining of 30 day old seedlings revealed a strong GUS signal in leaf vasculature, leaf rim, shoot apical meristem, and developing lateral roots (Figure 6). These sites, coincide with tissues known to accumulate high concentrations of auxin (Mattsson et al., 2003; Kerk et al., 2000). 

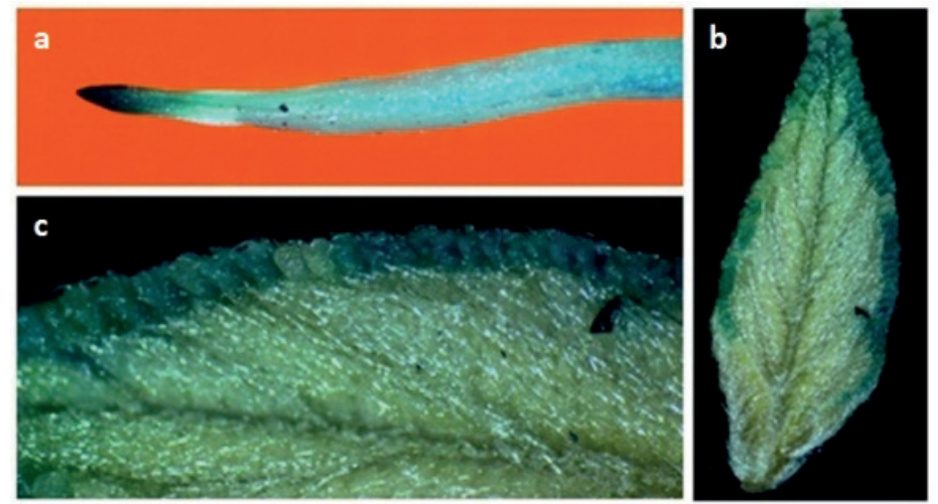

Figure 5. Bright field microscopy of the synthetic auxin-responsive reporter (DR5-GUS) expression in the root (a) and leaf $(b, c)$ of transgenic Trema tomentosa resulting in extensive blue staining.
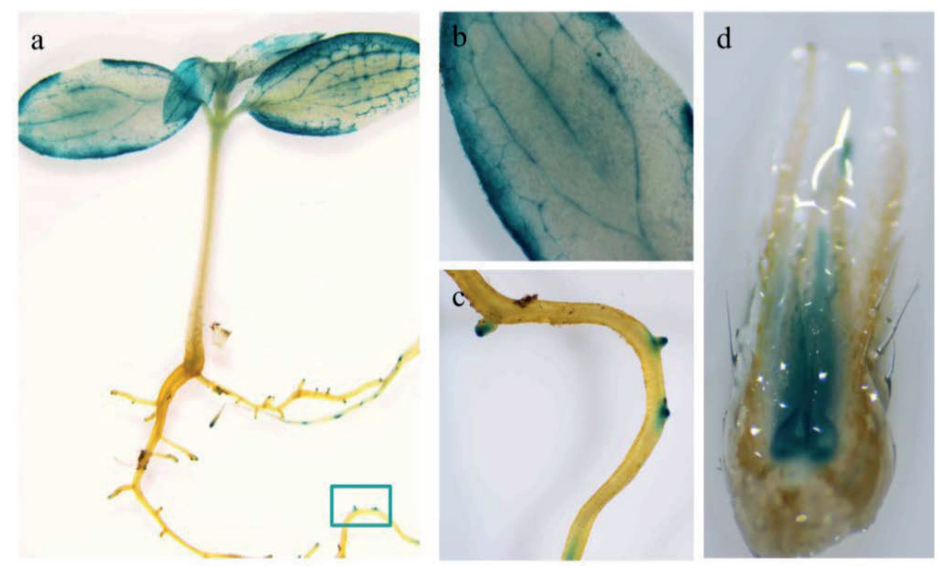

Figure 6. Bright field microscopy of the synthetic auxin-responsive reporter (DR5-GUS) expression in Trema tomentosa seedling (a-c) and apical meristem (d).

\section{Auxin accumulates in Trema during lateral root development}

For several plant species it is reported that lateral root initiation correlates with auxin accumulation (Casimiro et al., 2001). Since lateral root primordia generally occur in a regular pattern we made use of this phenomenon and examined auxin response during lateral root formation in T. tomentosa using a DR5::GUS line. First auxin was increased in inner cortical cells and in the underlying pericycle cell layer, even before the observation of cell divisions 


\section{Chapter 3}

(Figure 7a). After induction of pericycle divisions the blue staining increased in these cells (Figure 7b), which will give rise to the lateral root primordium formation (Figure 7c-e). Interestingly, the DR5 signal in the associated cortex cell layers remained and extended to the epidermis (Figure 7e), even though none of these cells divided during lateral root development.
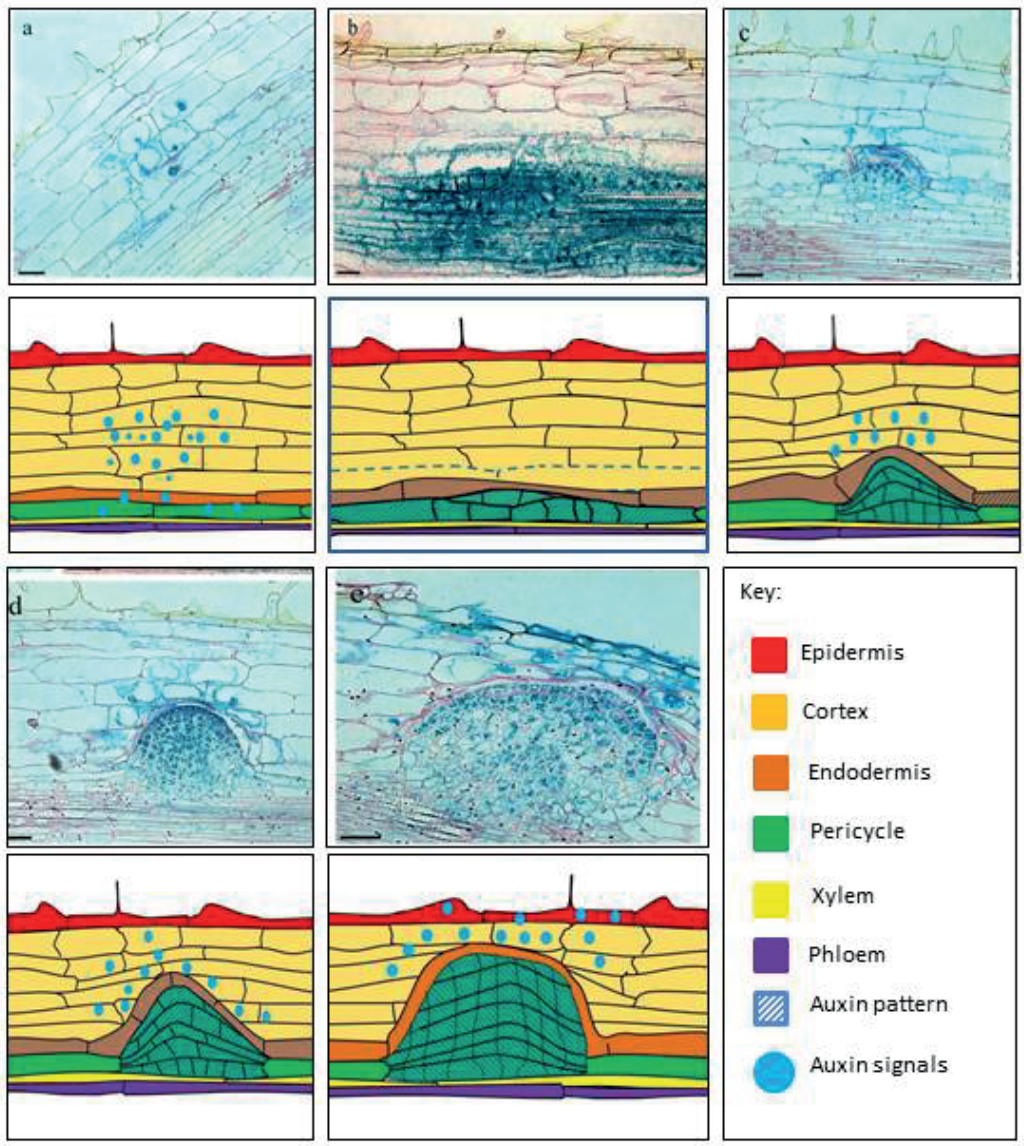

Figure7. Distribution of auxin during Trema tomentosa lateral root primordia development through spatial pattern of DR5::GUS expression during lateral root primordium initiation. (a) The DR5 activity gradient with the maximum at the primordium tip (inner cortical cell) is gradually established. (b) Cell division in pricycle and auxin accumulates in dividing cells (c-e) Auxin maximum in lateral root tip along with cell division. DR5 signals are visible in cortical and epidermal cell (d). Scale bars are: $25 \mu \mathrm{m}$. 


\section{Discussion}

Here we present an efficient and relatively fast method to obtain stably transformed plant lines of T. tomentosa. By using lateral leaf buds as starting material and an optimized single medium for callus and shoot formation, transgenic plants can be obtained within 6 months. As T. tomentosa can be propagated vegetatively in vitro, T0 plants can be effectively cloned and used for experimentation. Trema sp. is the closest relative of Parasponia and is unable to form rhizobium nitrogen fixing root nodules. Therefore the presented transformation protocol of $T$. tomentosa will be a new and effective tool in comparative studies to underpin the genetics of the rhizobium symbiosis.

T. tomentosa is a tropical tree species that grows best at temperatures of $>28^{\circ} \mathrm{C}$. However, in previous study we showed that T-DNA transfer of $A$. rhizogenes to $T$. tomentosa is most efficient at $21^{\circ} \mathrm{C}$ (Cao et al., 2012). In line with that we conducted co-cultivation at $21^{\circ} \mathrm{C}$ for 4 days, a temperature suboptimal for T. tomentosa growth. By doing so, transformation efficiencies up to $15 \%$ were achieved, which is sufficiently efficient to conduct experiments at larger scale.

Stable transformation offers four essential advantages over A. rhizogenes mediated root transformation. (I.) Transgenic lines can propagated, whereas this is not possible for compound plants carrying transgenic roots. In case of tree species however, generative propagation may be time consuming, or even impossible. However, in case of $T$. tomentosa this is not a problem, as this species flowers within 6 months and is self-compatible. Additionally, T0 plants can be vegetatively propagated. This further reduces the timespan between initial generation of transgenic plantlets and actual characterization of the transgenic line and subsequent phenotyping. (II.) An additional advantage of A. tumefaciens over A. rhizogenes is the absence of the transfer of the bacterial rol genes. This will allow conducting more reliably physiological studies. (III.) Also, stably transformed lines are suited for studies on shoot-root communication, which are impossible with chimeric compound plants that consist of a non-transgenic shoot carrying transgenic roots. In context of the rhizobium-symbiosis this is relevant as several studies indicated that shoot derived factors control root nodule formation (Mortier et al., 2010; Soyano et al., 2014). (IV.) A last advantage of stable transformation over A. rhizogenes is the possibility of re-transformation of an already transgenic line. In such case a different selection marker has to be used. 


\section{Chapter 3}

We introduced the auxin responsive reporter construct DR5::GUS into T. tomentosa. Using this reporter we detected transgene expression in meristematic regions in young developing leaves of primary transformed T0 plants. As the expression of the reporter is not affected upon vegetative propagation, it suggests that this in vitro propagation protocol is a fast way of propagating transgenic plants.

Root nodule organogenesis is associated with formation of a local auxin maximum in the plant root. By studying lateral root formation we showed that DR5::GUS can be used as auxin reporter also in T. tomentosa. Sectioning of young lateral root primordia revealed that DR5 activity is found not only in dividing pericycle cells that give birth to the primordium, but also in associated cortical cells that do not undergo cell divisions. This DR5 activity can be found even in the epidermal cells when the lateral roots start to emerge. This pattern is consistent with patterns reported in other species (Benková et al., 2003). In line with this, a T. tomentosa DR5::GUS line can be used as a tool to study Rhizobium LCO signaling in non-symbiotic species.

In conclusion, the presented protocol for T. tomentosa stable transformation can be an effective replacement of the transient $A$. rhizogenes transformation protocol. Stably transformed lines will be an essential tool to forward engineer rhizobium symbiosis.

\section{Materials and methods}

\section{Construction of DR5::GUS fusion construct}

pENTR ${ }^{\mathrm{TM}} / \mathrm{D}-\mathrm{TOPO}$ Cloning Kits (Invitrogen) and Gateway technology (Invitrogen) were used to generate the entry clone and genetic promoter-GUS construct (Karimi et al., 2002) of DR5::GUS construct, respectively. First, 14 synthetic DR5 DNA fragment repeats (Ulmasov et al., 1997) were inserted in the entry clone. Then, the entry vector was recombined into Gateway-compatible binary vector pKGW-RR, that contains GUS reporter and AtUBQ10::DsRED1 as a selection marker (Limpens et al., 2004), by using Gateway LR Clonase II enzyme mix (Invitrogen). Final construct was named pT14DR5.

\section{Plant transformation}

Apical meristems of Trema tomentosa plants were collected from the trees growing in the greenhouse and explants were divided in two groups. In one set apical meristems cut from the 
middle (parallel to growth axis) and the other, full meristems were used for stable transformation. Before cutting, all meristems surface sterilized in $4 \%$ hypochlorite (commercial bleach) for 10 min and subsequently washed 6 times with sterile MQ water. Agrobacterium tumefaciens strain $A G L 1$ carrying the DR5::GUS fusion constructs was used for transformations.

A. tumefaciens $A G L 1$ cultures harbouring construct pT14DR5 were grown overnight at $28^{\circ} \mathrm{C}$ on agar-solidified LB medium. The next day, A. tumefaciens was scraped from plate and resuspended in infiltration medium (McCown Woody Plant Medium (WPM) (Lloyd and McCown, 1980); Duchefa Biochemie, Haarlem, The Netherlands), 2.5 mg/L 6benzylaminopurine (BAP), $0.25 \mathrm{mg} / \mathrm{L}$ indole-3-butyric acid (IBA), $20 \mathrm{mg} / \mathrm{L}$ acetosyringone, $0.02 \%$ silvet L-77 (v/v)) at $\mathrm{OD}_{600}=0.6$. Sterilized $T$. tomentosa axillary buds were immersed in this medium for 30 minutes. Thereafter, they were transferred to co-cultivation medium (WPM, $2.5 \mathrm{mg} / \mathrm{L}$ 6-benzylaminopurine (BAP), $0.25 \mathrm{mg} / \mathrm{L}$ indole-3-butyric acid (IBA), $20 \mathrm{mg} / \mathrm{L}$ acetosyringone, 0.8\% Daichin agar (Duchefa Biochemie, Haarlem, The Netherlands) in $\varnothing 10 \mathrm{~cm}$ round petri dishes. Plates were incubated in darkness at $28^{\circ} \mathrm{C}$ for three days. After three days, explants were transferred to selective media containing cefotaxime $(300 \mathrm{mg} / \mathrm{L})$ and kanamycin $(50 \mathrm{mg} / \mathrm{L})$. Explants were cultured on three different selective media to determine optimal hormone concentrations for tissue regeneration. Composition of these three media is listed below. Explants were incubated at $28^{\circ} \mathrm{C}$ under a $16 / 8$ hour light/dark regime until regeneration was observed.

\section{Hormonal treatments}

Three different hormone combinations were performed to check the efficiency of callus, shoot and root regeneration.

$\mathrm{M} 1: \mathrm{WPM}+(2.5 \mathrm{mg} / \mathrm{l}) \mathrm{BAP}+(0.25 \mathrm{mg} / \mathrm{l}) \mathrm{IBA}$

$\mathrm{M} 2: \mathrm{WPM}+(1.0 \mathrm{mg} / \mathrm{l}) \mathrm{BAP}+(0.1 \mathrm{mg} / \mathrm{l}) \mathrm{IBA}$

$\mathrm{M} 3: \mathrm{WPM}+(0.5 \mathrm{mg} / \mathrm{l}) \mathrm{BAP}+(0.05 \mathrm{mg} / \mathrm{l}) \mathrm{IBA}$

\section{Regeneration of the plants}

All emerged callus from explants transferred to the new fresh regeneration medium and kept at $28^{\circ} \mathrm{C}$. Within 8 weeks shoots emerged from the axillaries buds callus. Shoots of $>1.0 \mathrm{~cm}$ in length were cut and transferred to rooting medium $(20 \mathrm{~g} / \mathrm{L}$ sucrose, $2.4 \mathrm{~g} / \mathrm{L} \mathrm{WPM}, \mathrm{pH}$ : 5.8) in 


\section{Chapter 3}

round $\varnothing 10 \mathrm{~cm}$ Petri dish. These plates were covered by aluminium foil (darkness induced roots) and kept in the same growth cabinet at $28^{\circ} \mathrm{C}$ for four weeks. Rooted shoots were kept on hormone free medium ( $\mathrm{pH} 5.8,20 \mathrm{~g} / \mathrm{L}$ sucrose, $2.4 \mathrm{~g} / \mathrm{L}$ WPM, 0.8\% Daichin agar) for three weeks before transferring to the pots in greenhouse.

\section{Microscopy and Histology analysis}

Transgenic roots and shoots were selected based on red fluorescence, using Leica MZIII fluorescence stereomicroscope (filter settings; excitation 565/30 and emission 620/60). The number of transformed roots was determined based on DsRed1 fluorescence.

For GUS activity, fresh lateral roots, young leaves and seedlings generated from the F1 seeds of Trema tomentosa plants were collected from greenhouse. All materials were immersed in GUS staining solution including $25 \mathrm{mM}$ sodium phosphate buffer ( $\mathrm{pH} 7.0), 2 \mathrm{mM}$ 5-bromo-4-choro-3indolylb- D-glucuronide cyclohexylamine salt (X-gluc), $0.5 \mathrm{mM}$ ferricyanide, $0.5 \mathrm{mM}$ ferrocyanide and $10 \mathrm{mM}$ EDTA, vacuumed for 30 minutes and then incubated at $37{ }^{\circ} \mathrm{C}$ for overnight. For bleaching the tissues, the GUS staining solution was rinsed with 20, 50 and $70 \%$ ethanol respectively for 10 minutes. Stained tissue was checked with Leica MZIII fluorescence stereomicroscope for GUS expression. Fixation of material including roots and apical meristems was performed for $24 \mathrm{~h}$ at $4{ }^{\circ} \mathrm{C}$ in $5 \%$ glutaraldehyde $(\mathrm{v} / \mathrm{v})$ and $3 \%$ sucrose $(\mathrm{w} / \mathrm{v})$ dissolved in phosphate buffer (pH 7.0). Subsequently an ethanol dehydration series was carried out.

\section{Root Microsectioning}

Transgenic roots were fixed in phosphate buffer solution (PBS) with $0.25 \%$ glutaraldehyde included. Vacuum was applied for 1-2 $\mathrm{h}$ until tissues sat on the bottom. The tissues were incubated at $4^{\circ} \mathrm{C}$ for overnight. After two times washing with PBS, dehydration steps were performed with $10 \%, 30 \%, 50 \%, 70 \%, 90 \%$ and $100 \%$ ETOH respectively for $10 \mathrm{~min}$ at room temperature for each step. Plastic infiltration was done in four steps, which included solution A (100ml Technovit7100, 1pack HrdnerI, 2.5ml PEG400):100\% ETOH in 1:3, 1:1, 3:1 ratio respectively for $30-60 \mathrm{~min}$ in room temperature and finally treated with $100 \%$ solution A for overnight at $4^{\circ} \mathrm{C}$. All materials were transferred into cupules and nodules located in the middle. Solution A was removed and polymerization solution (15ml Solution A, 1ml Hardener II) was added immediately. To remove air from the samples, cupules were covered with parafilm and 
left for overnight at room temperature. After polymerization, holders were put on the blocks and holding solution (technovit3040: 2part powder, 1 part liquid) was added from the hole located in the centre of holder and kept for $15 \mathrm{~min}$ at room temperature. Finally sectioning of root segments were performed using a microtome machine and the samples were analysed by microscopy (Leica) after staining with toluidine blue $(0.5 \%)$ buffer and washing with tap water for 5 minutes. 


\section{Chapter 3}

\section{References}

Akkermans, A., et al. (1978). "N2-fixing root nodules in Ulmaceae: Parasponia or (and) Trema spp.?" Plant and Soil 49(3): 711-715.

Behm, J. E., et al. (2014). "Parasponia: a novel system for studying mutualism stability." Trends in Plant Science 19(12): 757-763.

Benková, E., et al. (2003). "Local, efflux-dependent auxin gradients as a common module for plant organ formation." Cell 115(5): 591-602.

Cao, Q., et al. (2012). "Efficiency of Agrobacterium rhizogenes-mediated root transformation of Parasponia and Trema is temperature dependent." Plant Growth Regulation 68(3): 459-465.

Casimiro, I., et al. (2001). "Auxin transport promotes Arabidopsis lateral root initiation." The Plant cell 13(4): 843852.

Den Camp, R. O., et al. (2011). "LysM-type mycorrhizal receptor recruited for rhizobium symbiosis in nonlegume Parasponia." Science 331(6019): 909-912.

Granqvist, E., et al. (2015). "Bacterial-induced calcium oscillations are common to nitrogen-fixing associations of nodulating legumes and non-legumes." New Phytologist 207(3): 551-558.

Karimi, M., et al. (2002). "GATEWAYTM vectors for $<\mathrm{i}>$ Agrobacterium $</ \mathrm{i}>$-mediated plant transformation." Trends in Plant Science 7(5): 193-195.

Kerk, N. M., et al. (2000). "Auxin metabolism in the root apical meristem." Plant Physiology 122(3): 925-932.

Limpens, E., et al. (2004). "RNA interference in Agrobacterium rhizogenes-transformed roots of Arabidopsis and Medicago truncatula." Journal of Experimental Botany 55(399): 983-992.

Mattsson, J., et al. (2003). "Auxin signaling in Arabidopsis leaf vascular development." Plant Physiology 131(3): 1327-1339.

Mortier, V., et al. (2010). "CLE peptides control Medicago truncatula nodulation locally and systemically." Plant Physiology 153(1): 222-237.

Op den Camp, R. H., et al. (2012). "Nonlegume Parasponia andersonii deploys a broad rhizobium host range strategy resulting in largely variable symbiotic effectiveness." Molecular Plant-Microbe Interactions 25(7): 954-963.

Roberts, N. J., et al. (2013). "Rhizobial and mycorrhizal symbioses in Lotus japonicus require lectin nucleotide phosphohydrolase, which acts upstream of calcium signaling." Plant Physiology 161(1): 556-567.

Soyano, T., et al. (2014). "NODULE INCEPTION creates a long-distance negative feedback loop involved in homeostatic regulation of nodule organ production." Proceedings of the National Academy of Sciences 111(40): 14607-14612.

Ulmasov, T., et al. (1997). "Aux/IAA proteins repress expression of reporter genes containing natural and highly active synthetic auxin response elements." The Plant Cell Online 9(11): 1963-1971.

Wang, H., et al. (2009). "Rosid radiation and the rapid rise of angiosperm-dominated forests." Proceedings of the National Academy of Sciences 106(10): 3853-3858.

Yang, M.-Q., et al. (2013). "Molecular phylogenetics and character evolution of Cannabaceae." Taxon 62(3): 473485 . 


\section{Chapter4}

Exogenous Nitrate Interferes with the Switch from Infection to Fixation Thread Formation in Parasponia andersonii Root Nodules

Maryam Seifi Kalhor ${ }^{1}$, Elena Fedorova ${ }^{1}$, Jan Verver $^{1}$, Emma Granqvist ${ }^{2}$, Giles Oldroyd ${ }^{2}$, Robin van Velzen $^{1}$, Ton Bisseling ${ }^{1}$ and René Geurts ${ }^{1}$

This chapter has been submitted for publication

1. Laboratory of Molecular Biology, Department of Plant Science, Wageningen University, Droevendaalsesteeg 1, 6708PB Wageningen, The Netherlands.

2. John Innes Centre, Norwich Research Park, Norwich NR4 7UH, UK. 


\section{Chapter 4}

\section{Abstract}

Parasponia trees are the only non-legume plant species that can form nitrogen fixing nodules with rhizobium bacteria. In legumes, rhizobium-induced nodule formation is tightly controlled and inhibited by exogenous fixed nitrogen. Species in the Parasponia lineage gained the rhizobium symbiosis trait more recent when compared to legumes. We questioned whether similar to legumes also Parasponia acquired mechanisms to control its symbiotic interaction in presence of exogenous fixed nitrogen. To get insight in the mechanism controlling nitrogen fixing symbiosis in presence of exogenous nitrogen, we studied nodule formation and intracellular rhizobium infection in Parasponia andersonii supplied with doses of $\mathrm{KNO}_{3}$ ranging from 0.2 to $50 \mathrm{mM}$. We found that nodule primordium formation and intracellular infection have different sensitivities to nitrates. $P$. andersonii formed nodules at all tested nitrate concentrations, whereas progression of intracellular infection was impaired at $20 \mathrm{mM}$. Specifically, the switch from infection to fixation threads was affected, a process known to be controlled by the LysMtype receptor PaNFP. However, using PaNFP knockdown mutants and LCO responsive $\mathrm{Ca}^{2+}$ oscillation experiments indicate that exogenous nitrate doesn't affect LCO signaling. We conclude that $P$. andersonii employs a novel mechanism to control intracellular rhizobium colonization in presence of exogenous nitrates. 


\section{Introduction}

Fixed nitrogen is an essential nutrient for plant growth, but in most ecosystems its availability is limited. Some plants species -like most legumes (Fabaceae) and species of the genus Parasponia- have overcome this problem by establishing a root nodule symbiosis with a diverse range of nitrogen fixing soil bacteria that are collectively known as rhizobia (Willems, 2006). Parasponia species and legumes obtained their symbiotic traits by convergent evolution at different moments in time. The nitrogen-fixing symbiosis trait in legumes is estimated to be $\sim 60$ million years old (Lavin et al., 2005; Sprent, 2008; Doyle, 2011). In case of Parasponia the evolutionary origin of the nitrogen-fixing rhizobium symbiosis is not dated, however, two lines of evidence strongly suggests that the symbiosis trait in this lineage is relatively young when compared to legumes. First, the Parasponia genus is nested within -and only recently diverged from- the genus Trema (Yang et al., 2013; Werner et al., 2014). Second, the Parasponiarhizobium symbiosis is far less sophisticated when compared to the legume-rhizobium symbiosis (Behm et al., 2014). In case of legumes the relative long period of evolution has shaped the symbiosis trait in such a way that it has become highly efficient and robust. Legumes have gained mechanisms that link the N-status of the plant to its susceptibility for rhizobium. For example, in most legume species nodule formation is inhibited by moderate concentrations of exogenous fixed-nitrogen sources (Streeter, 1988). Such mechanism enables legume plants to exploit exogenous nitrogen sources, rather than to maintain an energetically costly symbiotic relation with rhizobium. As the Parasponia-rhizobium symbiosis evolved more recent and independent from legumes, we questioned to what extend similar mechanisms do occur in these species.

The negative effects of soil nitrates on symbiotic nitrogen fixation in legumes are known for a long time (Streeter, 1988). In model legumes Lotus japonicus and Medicago truncatula root nodule formation is fully inhibited by $5-10 \mathrm{mM}$ exogenous fixed nitrogen (Sagan et al., 1995; Barbulova et al., 2007). Effects of exogenous nitrates can be categorized in three types of responses that differ in sensitivity; (i) reduced infection rates, (ii) decreased nitrogen fixation rates and (iii) decreased nodule mass or even total absence of nodules. Of these, infection seems to be least sensitive to exogenous nitrates (Streeter, 1988). 


\section{Chapter 4}

In legumes, bacterial infection and nodule primordium formation are tightly controlled by rhizobium induced lipo-chitoolichosaccharide (LCO) signaling. These rhizobial signals are perceived by a specific heterodimeric complex of two types of LysM domain containing receptor kinases, named LjNFR1/MtLYK3 and LjNFR5/MtNFP in L. japonicus and M. truncatula (Broghammer et al., 2012; Moling et al., 2014). These receptors subsequently trigger a plethora of symbiotic responses, ranging from nuclear $\mathrm{Ca}^{2+}$ oscillation, symbiotic gene expression, changes in the cytokinin and auxin homeostasis, root hair growth responses, and nodule primordium formation (Oldroyd, 2013; Miri et al., 2016). Studies in different legume species showed that read-out of LCO signaling at least in part is affected by exogenous fixed nitrogen. For example, in Vicia sativa it was shown that LCO induced root hair growth responses are affected (Heidstra et al., 1994), whereas studies in M. truncatula and L. japonicus revealed a strong inhibition of NIN expression; a key regulatory gene of bacterial infection and root nodule formation (Schauser et al., 1999; Barbulova et al., 2007; Marsh et al., 2007).

In Parasponia species rhizobium bacteria enter the roots via crack entry. First upon reaching the dividing cells that form the nodule primordium, intracellular infection is achieved (Lancelle and Torrey, 1984). These infection threads have a similar appearance as in legumes. However, in contrast to most legumes the bacteria are not released as symbiosomes in the cytoplasm, but remain bound in so-called fixation threads. Fixation threads distinct from infection threads by relatively thin cell walls and more loosely packed bacteria (Price et al., 1984). Similar to legumes, P.andersonii nodule primordium formation is triggered upon perception of rhizobium LCOs (Marvel et al., 1987; Op den Camp et al., 2011). The putative ortholog of LjNFR5/MtNFP in P.andersonii -PaNFP- was found to fulfil a symbiotic function. Knockdown of PaNFP by RNAi resulted in reduced nodulation efficiency, whereas the few nodules formed on PaNFP RNAi roots are defective in fixation thread formation (Op den Camp et al., 2011). Taken together, the symbiotic functioning of LjNFR5/MtNFP orthologous genes in legumes as well in P.andersonii suggests that genetic constraints have guided evolution of rhizobium symbiosis in both lineages (Streng et al., 2011; Geurts et al., 2012).

It remains elusive whether P.andersonii gained additional mechanisms similar to control the interaction with rhizobium. Putting forward the relative short period of time in evolution when compared to legumes, we questioned to what extend P.andersonii gained a mechanism to control its symbiotic nitrogen fixing partner in presence of exogenous nitrates. By studying root nodule 
formation at different doses of exogenous nitrate we found that $P$. andersonii employs a novel mechanism to control the living space of its endosymbiotic microbe by inhibiting formation of fixation threads.

\section{Results}

\section{Dose-response relationship of $P$. andersonii nodulation efficiency and exogenous nitrate}

In legumes, exogenous fixed-nitrogen acts on nodule initiation, infection and nitrogen fixation activity and the severity of the inhibition varies among species (Harper and Gibson, 1984; Streeter, 1988; Carroll and Mathews, 1990). To determine whether nodulation of Parasponia also is inhibited by nitrate, $P$. andersonii plantlets inoculated with Bradyrhizobium elkanii WUR3 were grown at 5 different $\mathrm{KNO}_{3}$ concentrations $(0.2,1,5,10$ and $20 \mathrm{mM})$. Plants were harvested at 8 weeks post inoculation. Highest shoot mass was obtained when the plant medium was supplemented with $20 \mathrm{mM} \mathrm{KNO}_{3}$, whereas root biomass was highest when plants were grown at $\mathrm{KNO}_{3}$ concentrations of $10 \mathrm{mM}$ (Fig. 1A,B). This suggests that $P$. andersonii plants reduce their investment in root biomass at $\mathrm{KNO}_{3}$ concentrations above $10 \mathrm{mM}$. Next, we determined the nodulation efficiencies of $P$. andersonii plants grown at the different doses of $\mathrm{KNO}_{3}$. This showed that up to $10 \mathrm{mM}$ nitrate, a positive effect on nodulation can be observed. Plants supplemented with this concentration had a 5 fold higher number of nodules when compared to plants that were grown with $0.2 \mathrm{mM} \mathrm{KNO}_{3}$. At higher nitrate concentrations (20 $\left.\mathrm{mM} \mathrm{KNO}_{3}\right)$ nodulation efficiencies markedly drop ( 90\%) (Fig. 1C). However, at this concentration still up to 20 nodules per plant were formed, a nodulation efficiency comparable to plants grown on a hundred lower concentration $(0.2 \mathrm{mM})$. 


\section{Chapter 4}

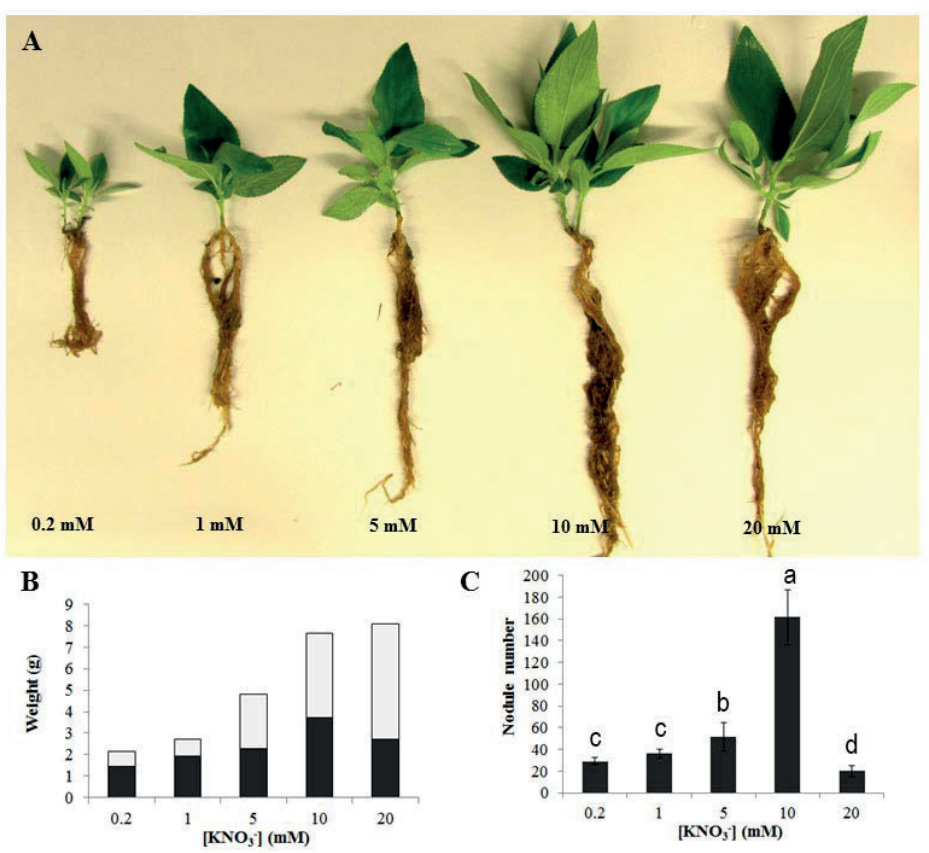

Figure 1. Effects of exogenous nitrate on Parasponia andersonii plants inoculated with Bradyrhizobium elkanii WUR3 and grown on different concentrations of $\mathrm{KNO}_{3}$. (A) Plant phenotypes, and (B) shoot (white bar) and root (black bar) fresh weights 8 weeks post inoculation. (C) Average number of nodules per plant. ( $\mathrm{n}=10,8$ weeks post inoculation).

To determine whether nodulation can be fully inhibited at higher concentrations of exogenously nitrate, we grew $P$. andersonii plants with 30 and $50 \mathrm{mM}$ exogenous $\mathrm{KNO}_{3}$. In such high doses of nitrate plant fresh weights marginally increased (Fig. S1B). Interestingly, the $P$. andersonii grown at 30 or $50 \mathrm{mM} \mathrm{KNO}_{3}$ still formed a few root nodules, although less when compared to plants grown at lower nitrate concentrations (Fig. S1A). Taken together, it shows that $P$. andersonii plants can control root nodule initiation in response to high exogenous nitrate. However, $P$. andersonii is unable to fully block this developmental process that is triggered in response to rhizobia, even not if nitrogen seems no longer growth limiting factor.

\section{Nitrate interferes with fixation thread formation}

We noted that the nodule fresh weight negatively correlated with the exogenous nitrate concentration supplied to the plants (Fig. 2A). Therefore, we examined the effect of nitrate on 
the nitrogen fixation capacity of nodules by measuring nitrogenase activity. This showed that nitrogenase activity decreased in a nitrate concentration-dependent manner. Nitrogenase activities decreased by 5 and 23-fold, respectively, in nodules isolated from plants supplied with 10 and $20 \mathrm{mM}$ exogenous $\mathrm{KNO}_{3}{ }^{-}$, when compared to nodules of plants grown at $0.2 \mathrm{mM} \mathrm{KNO}_{3}^{-}$ (Fig. 2B). These results indicate that although $P$. andersonii is capable to form nodules in presence of a high concentration of exogenous nitrate, these nodules have lower nitrogen fixation efficiency.
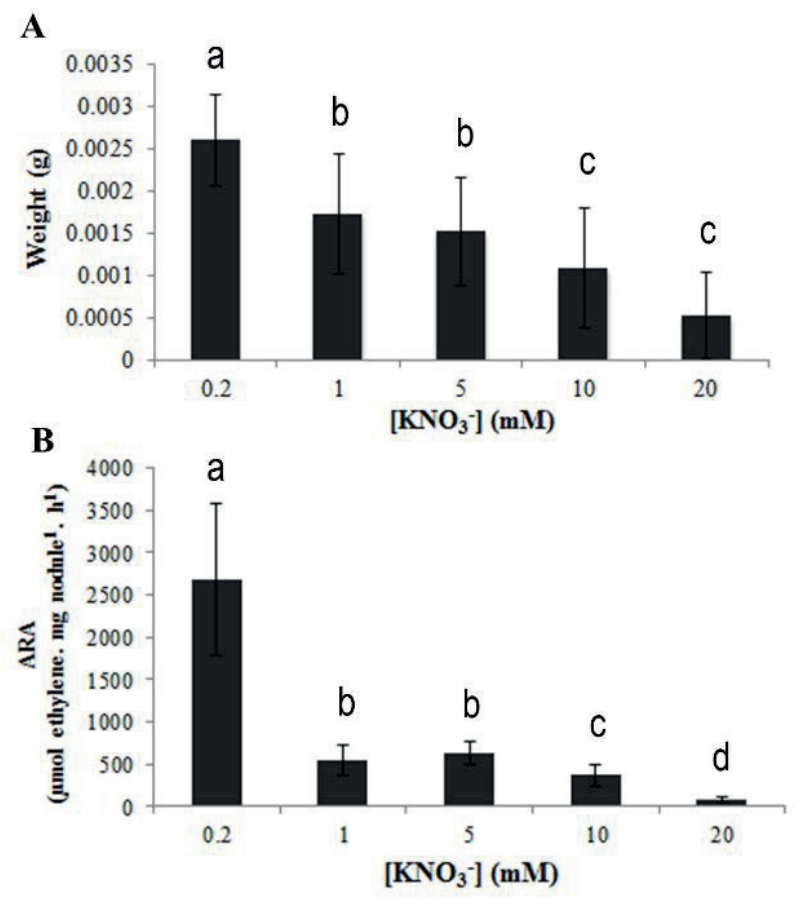

Figure 2. (A) Fresh weight of individual Parasponia andersonii nodules and (B) nitrogenase activity measured by acetylene reduction rates of nodules grown at different $\mathrm{KNO}_{3}$ concentrations and inoculated with Bradyrhizobium elkanii WUR3. ( $\mathrm{n}=10$ plants / treatment).

We examined the nodule cyto-architecture to determine whether exogenous nitrate affects nodule development. Nodules grown on $0.2 \mathrm{mM}$ and $20 \mathrm{mM}$ were sectioned and studied by light and 


\section{Chapter 4}

transmission electron microscopy (TEM) (Fig. 3 and Fig. 4). The general anatomy of the nodules formed was similar irrespective of the exogenous nitrate concentration. As P.andersonii nodules have an apical meristem a gradient of developmental stages of infected cells could be distinguish along the longitudinal axis. Inward nodule cortical cells that surrounded the vascular bundle were infected, whereas outward nodule cortical cells remained uninfected (Fig. 3A). Cells directly adjacent to the meristem contained newly infected cells with infection threads (Fig. 3B). Using TEM it was noted that intracellular infection started from an apoplastic micro-colony that formed a cell wall bound infection thread (Fig. 4A). Such infection threads contained a single file of bacteria. Further penetration of these infection threads into the host cells coincided with fragmentation of the main vacuole (Fig. 4B). Infection threads eventually changed shape and became wider, now harbouring 2-3 files of bacteria (Fig. 4B, C). TEM studies revealed that at this stage the infection structures were largely deprived of a cell wall matrix; a hallmark for fixation threads (Compare Fig. 4A,B and Fig.4C) (Price et al., 1984). This change in infection morphology reflects the switch to the symbiotic stage.

Nodules formed in presence of $20 \mathrm{mM}$ exogenous nitrate showed dramatic difference in infection (Compare Fig. 3A and 3D). Initial infection of nodule cortical cells was similar to nodules grown at $0.2 \mathrm{mM} \mathrm{KNO}_{3}$. However, further development of intracellular infection threads towards fixation threads was seriously hampered (Fig. 3D-F, Fig 4D). Infected cells of nodules grown at $20 \mathrm{mM} \mathrm{KNO}_{3}$ contained only infection threads, which remained relatively short and bounded in a dense cell wall (Fig. 3F, Fig 4E). Central vacuoles in these cells do not undergo fragmentation and remained intact (Fig. 4E). 

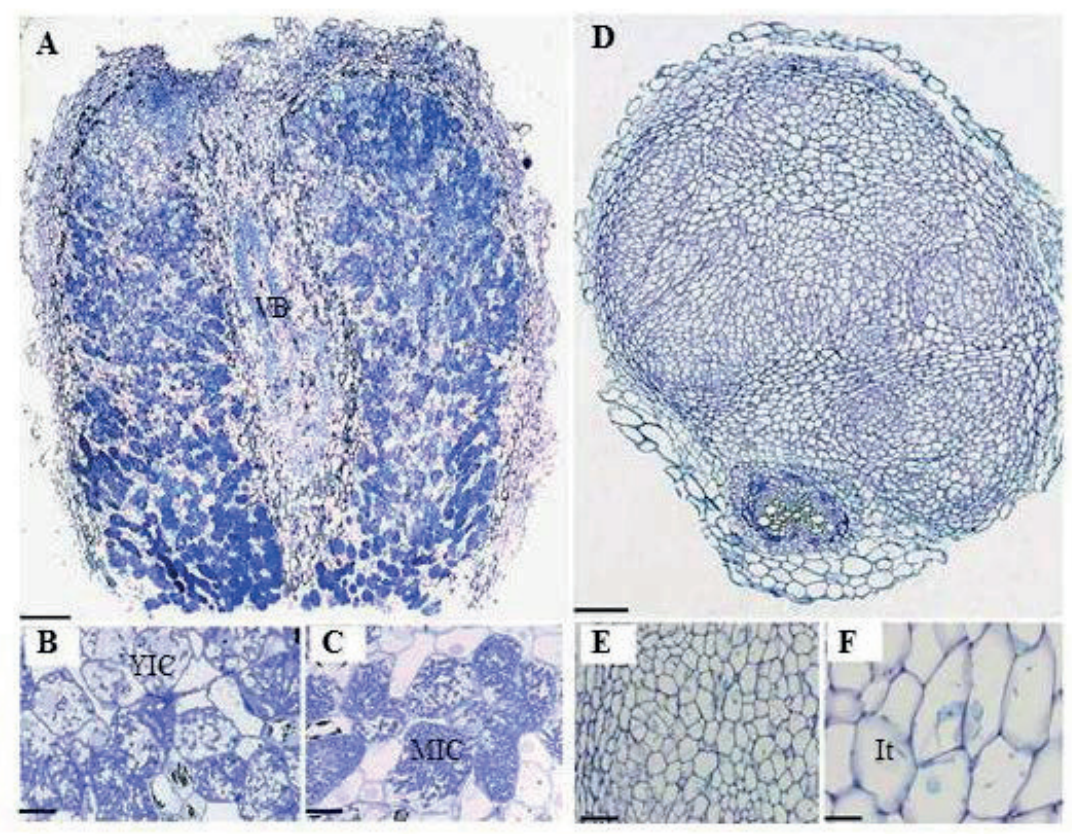

Figure 3. Sections of Parasponia andersonii nodules infected with Bradyrhizobium elkanii WUR3 and grown at 0.2 (A-C) or $20 \mathrm{mM}$ (D-F) $\mathrm{KNO}_{3}$. (A) Longitudinal section of a nodule formed at $0.2 \mathrm{mM} \mathrm{KNO}_{3}$ with a central vascular bundle (VB). (B) Apical region of the nodule containing recently infected cells with infection threads and young infected cells (YIC). (C) Infected cells filled with fixation threads and mature infected cells (MIC). (D) Longitudinal section of a nodule formed at $20 \mathrm{mM} \mathrm{KNO}_{3}$. (E) Apical region of the nodule containing recently infected cells with infection threads. (F) Cells in the central zone of the nodule containing only infection threads (It), but no fixation threads. Bars: A, D: $75 \mu \mathrm{m}, \mathrm{B}, \mathrm{C}: 12,5 \mu \mathrm{m}, \mathrm{E}: 25 \mu \mathrm{m}$ F: $10 \mu \mathrm{m}$. 


\section{Chapter 4}
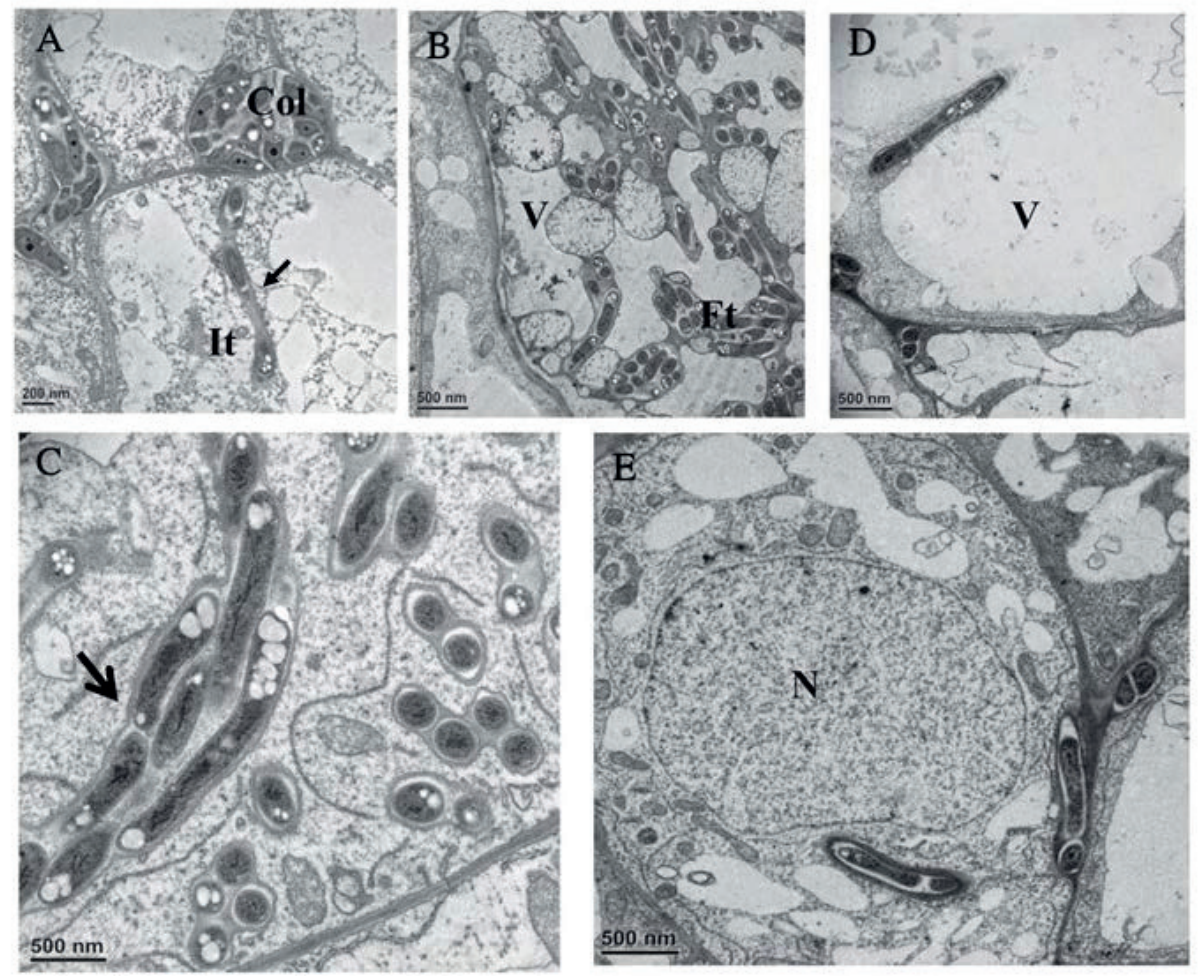

Figure 4. Transmission electron microscopy images of infected cells of Parasponia andersonii nodules infected with Bradyrhizobium elkanii WUR3 and grown at 0.2 (A-C) or $20 \mathrm{mM}(\mathbf{D}, \mathbf{E}) \mathrm{KNO}_{3}$. (A) Infection thread with one file of rhizobia entering to the cell from the bacterial colony situated in apoplast. Note presence of cell wall of infection thread (arrow). (B) Cell containing infection threads with one file of bacteria, and fixation threads containing two files of bacteria. Note the vacuole fragmentation in cell. (C) Thin cell wall of fixation threads (marked by arrow). (D, E) The structure of nodules treated with $20 \mathrm{mM}$ nitrate. (D) Infection threads entering a young nodule cell containing only small vacuoles. (E) Mature infected cell with developed vacuole containing only an infection thread. Col: bacteria colony in apoplast. It: infection thread, Ft: fixation thread, V: vacuole, N: nucleus.

Taken together, these data show that high fixed nitrogen does not prohibit nodule initiation and intracellular infection thread formation, but hampers formation of fixation threads. This block of intracellular infection coincides with occurrence of thick (and possibly rigid) infection thread cell walls, which may be the cause of infection suppression. This suggests that P.andersonii employs an active mechanism to control intracellular rhizobial colonization in case alternative sources of fixed nitrogen are available. 


\section{Exogenous nitrate doesn't interfere with LCO signaling in P. andersonii}

In a previous study we showed that fixation thread formation in $P$. andersonii is controlled by the LysM-type receptor kinase PaNFP (Op den Camp et al., 2011). PaNFP is orthologous to the rhizobium LCO receptors LjNFR5/MtNFP in L. japonicus and M. truncatula. In M. truncatula it was shown by nodule specific knock down that MtNFP controls the switch from infection thread growth to bacterial release (Moling et al., 2014). As in legumes LCO signaling is sensitive to exogenous nitrate (Heidstra et al., 1994, 1997; Barbulova et al., 2007; Marsh et al., 2007), we tested whether this is the case for P.andersonii as well. To investigate this, two experiments were conducted. First it was determined whether LCO-induced $\mathrm{Ca}^{2+}$-oscillation response is affected by exogenous nitrate. To test this, $P$. andersonii plantlets carrying transgenic roots expressing the NupYC2.1 $\mathrm{Ca}^{2+}$ reporter were generated (Granqvist et al., 2015). Compound plantlets carrying transgenic roots were grown on EKM-medium complemented with either $0.2 \mathrm{mM}$ or $20 \mathrm{mM}$ $\mathrm{KNO}_{3}$. Subsequently, transgenic roots -selected based on green fluorescence- were treated with an LCO mixture of Sinorhizobium fredii NGR234 $\left(\sim 10^{-9} \mathrm{M}\right) . \mathrm{Ca}^{2+}$-oscillation could be observed in about $50 \%$ of the tested epidermal cells, irrespective of the nitrate concentration in the growth medium (Fig. 5). Likewise, the spiking frequency and amplitude is undistinguishable between both nitrate regimes. This suggests that inhibitory effect of exogenous nitrate acts independent or downstream of- rhizobium LCO induced $\mathrm{Ca}^{2+}$ spiking.

A

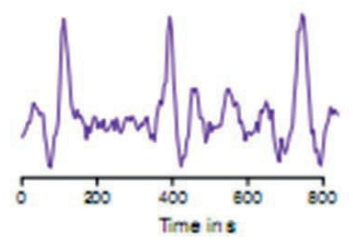

B

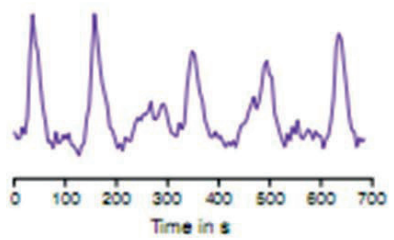

Figure 5. $\mathrm{Ca}^{2+}$ oscillation in Parasponia andersonii root hairs grown in $0.2 \mathrm{mM} \mathrm{KNO}_{3}(\mathrm{~A})$ and $20 \mathrm{mM} \mathrm{KNO}_{3}$. Roots transformed with NupYC2.1 and treated with an LCO mixture of Sinorhizobium fredii NGR234 $\left(10^{-9} \mathrm{M}\right)$.

In a second experiment we determined whether exogenous nitrate may interfere with PaNFP functioning. First we determined whether expression of PaNFP is affected by exogenous nitrate. qRT-PCR using RNA isolated from roots of the plants grown on 0.2 and $20 \mathrm{mM} \mathrm{KNO}_{3}$ showed that expression of PaNFP is not affected by exogenous nitrate (Fig. S2). Next it was determined 


\section{Chapter 4}

whether exogenous nitrate has an additive effect on the nodulation and infection phenotype of PaNFP knocked down roots. We have generated transgenic plants harbouring a PaNFP RNAi construct. Transgenic roots displayed a reduction of PaNFP mRNA of $>80 \%$ (Fig. S3). Transgenic plants harbouring either PaNFP RNAi or an empty vector construct were grown on either 0.2 or $20 \mathrm{mM} \mathrm{KNO}$ for 2 weeks, and subsequently inoculated with Bradyrhizobium elkanii WUR3. PaNFP RNAi roots formed significantly less nodules when compared to the empty vector control, and these nodules remained relatively small. However, nodulation efficiency of PaNFP RNAi plants was independent of the nitrate regime (Fig. 6).

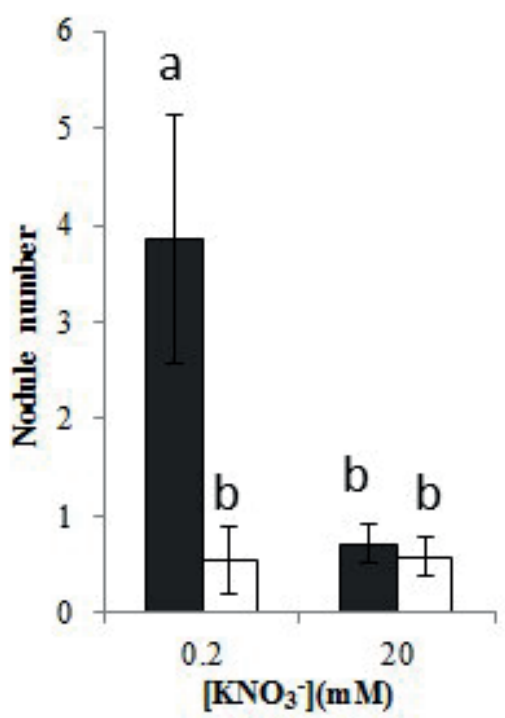

Figure 6. Nodulation efficiency of PaNFP RNAi knocked down roots (white bars) and control roots (black bars) at 0.2 or $20 \mathrm{mM} \mathrm{KNO}_{3}$ (eight weeks post inoculation, Bradyrhizobium elkanii WUR3).

To determine whether the infection phenotype of these PaNFP RNAi nodules is affected by the nitrate regime, the structure of the nodules was studied by light microscopy (Fig.7). This showed that some, but not all cells in transgenic nodules were infected, but infection threads remain short and infected cells contained large vacuoles (Fig. 7C, H). This phenotype was irrespective of the nitrate regime (Fig. 7C-E and F-H), indicating that PaNFP and exogenous nitrate do not display 
additive effects. Taken-together, these studies reveal that exogenous nitrate acts downstream or independent of PaNFP and LCO induced $\mathrm{Ca}^{2+}$-oscillation signaling.
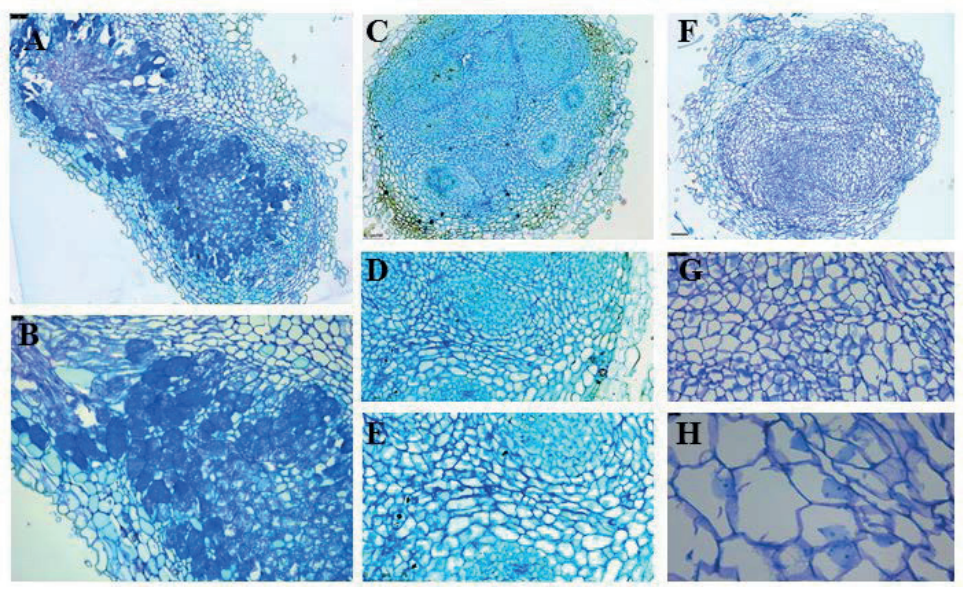

Figure 7. Sections of PaNFP knockdown nodules grown at $0.2 \mathrm{mM} \mathrm{KNO3} \mathrm{(C-E)} \mathrm{or} 20 \mathrm{mM} \mathrm{KNO3} \mathrm{(F-H)} \mathrm{compare}$ with non-transgenic control plants grown in $0.2 \mathrm{mM}(\mathbf{A}, \mathbf{B})$. Magnifications $(\mathbf{D}, \mathbf{E}$ and $\mathbf{G}, \mathbf{H})$ show that in infected

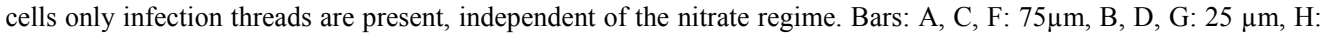
$\mu \mathrm{m} 12.5$, E: $10 \mu \mathrm{m}$.

\section{Discussion}

In legumes, exogenous nitrate has severe effects on the rhizobium symbiosis; ranging from premature nodule senescence, inhibition of nodule growth, inhibition of formation of new nodule primordia, reduced bacterial infection and reduced expression of -at least some- LCO-responsive genes. The severity of these responses vary among legume species (Harper and Gibson, 1984), suggesting that exogenous nitrate induced inhibition of nodulation. This represents an adaptation in the symbiotic trait which in part evolved independent from different legume lineages. Here we investigated the effect of exogenous nitrogen on the Parasponia-rhizobium symbiosis, which in evolutionary terms is relatively young. We showed that in presence of exogenous nitrate formation of fixation threads -the intracellular structure allowing exchange of nutrients- is most sensitive and inhibited at nitrate concentrations of $\sim 20 \mathrm{mM}$. This suggests that Parasponia 


\section{Chapter 4}

employs an active mechanism to control intracellular rhizobial colonization when alternative sources of fixed nitrogen are available.

In P.andersonii also the nodulation efficiency is affected by exogenous nitrate. Surprisingly, nodulation efficiency is highest at $10 \mathrm{mM} \mathrm{KNO}_{3}$, a concentration that in case of legumes generally is found to be inhibitory. At higher $\mathrm{KNO}_{3}$ concentrations the nodulation efficiency of P.andersonii drops, but unlike many legumes nodulation is not inhibited completely, even in presence of $50 \mathrm{mM} \mathrm{KNO}_{3}$. Strikingly, the few nodules that are formed contain many infected cells, suggesting that infection thread formation is not affected by high concentrations of exogenous $\mathrm{KNO}_{3}$. Instead, the switch from infection threads with relative dense cell walls to fixation threads that are largely devoid from cell wall material is blocked. In nodules formed in presence of relative low exogenous nitrates, this developmental switch in intracellular infection structure occurs in the first 10-15 cell layers proximal to the nodule meristem. These cells enlarge in size when compared to uninfected cells. Formation of fixation threads coincides with fragmentation of the vacuole similar as seen in legumes (Gavrin et al., 2014). However, in presence of exogenous nitrate, the vacuoles remain large, infection threads stop growing and do not progress to form a fixation threads. Taken-together, this indicates that nitrate-grown $P$. andersonii plants block specifically the cellular process underlying formation of an interface with reduced cell wall, which allows better nutrient exchange between both partners.

Fixation thread formation in P. andersonii nodules is controlled by the putative LCO receptor PaNFP (Op den Camp et al., 2011). Most legumes do not form fixation threads, but rather release the rhizobia in a small membrane compartment, which are known as symbiosomes. These symbiosomes originate from cell wall free regions of infection threads. Studies in legumes revealed that several key genes in the rhizobium LCO signaling network are essential to commit this process. For example, in $M$. truncatula a reduced expression of the symbiotic receptors MtNFP, MtDMI2 as well as a knockout mutation in MtIPD3 results in absence of a cell wall free region in the infection threads and a block on symbiosome formation (Limpens et al., 2005; Horváth et al., 2011; Ovchinnikova et al., 2011; Moling et al., 2014). We tested the functional relation between $P a N F P$ and exogenous nitrate in controlling fixation thread formation. Nodule infection phenotypes of PaNFP knockdown or wild type plants grown under high exogenous nitrate concentration are very comparable, suggesting that fixation thread formation is a strictly controlled in the rhizobium-Parasponia symbiosis. In legumes several studies have been 
conducted to place the inhibitory effect of exogenous fixed-nitrogen in relation to rhizobium LCO signaling. The outcome of these studies varied depending on the species and which response was investigated. For example, in Vicia sativa it was shown that inhibition of LCOinduced root hair growth responses has a 24-36 h lag phase post combined nitrogen application (20 mM) (Heidstra et al., 1994). Under such growth conditions, LCO-induced gene expression shows differential response to exogenous fixed nitrogen were some genes are still induced (e.g. $V_{S L B 1}$ ), whereas for others symbiotic expression ceased (VsENOD5 and $V_{S} E N O D 12$ ) (Heidstra et al., 1997). Studies in L. japonicus revealed that inhibition of LCO-induced root hair growth responses is mainly triggered by ammonium, whereas nitrate interferes with symbiotic gene expression (e.g. LjNIN) (Barbulova et al., 2007). Similarly, repression of MtNIN in presence of nitrate was reported for M. truncatula (Marsh et al., 2007). We performed three types of experiments to elucidate the effect of exogenous nitrate on LCO signaling in P. andersonii; (I) LCO induced $\mathrm{Ca}^{2+}$ oscillation in root hairs, (II) PaNFP expression in roots and (III) additive effect on the PaNFP knock down phenotype. None of these experiments supported the idea that exogenous nitrate affects LCO signaling and/or PaNFP functioning.

Besides in P.andersonii root nodules, fixation threads form also in few legume species -e.g. Andira sp., Chamaecrista ensiformis and C. flexuosa- as well as in the nitrogen fixing symbiosis between filamentous actinobacteria of the genus Frankia and a small polyphyletic group of nonlegume plant species (known as Actinorhizal plants) (Faria et al., 1986; Naisbitt et al., 1992; Pawlowski and Demchenko, 2012). In case of Actinorhizal symbiosis the effect of exogenous fixed nitrogen has been studied. These showed that fixed nitrogen affects nodule formation, nodule biomass and $\mathrm{N}_{2}$-fixation, very similar as described for legumes (Huss-Danell, 1997). Also variation in sensitivity between different Actinorhizal species is observed. e.g. for Alnus glutinosa, Casuarina cunninghamiana and Myrica cerifera exogenous nitrate in concentration of $1 \mathrm{mM}$ is inhibitory, whereas for Elaeagnus angustifolia no inhibitory effect is reported for concentrations up to $3 \mathrm{mM}$ nitrate (Kohls and Baker, 1989). As the actinorhizal symbiosis is considered to be tens of million years old and possibly evolved multiple times independently in different lineages (Doyle, 2011; Werner et al., 2014; Li et al., 2015), the observed variation may reflect lineage-specific adaptations. Alternatively, it has been shown that the difference in sensitivity to exogenous nitrate correlates with the employed infection strategy of the plant. Actinorhizal plants with root hair based infection are more sensitive to the inhibitory effects of 


\section{Chapter 4}

nitrate than plants infected by crack entry (Kohls and Baker, 1989). This hypothesis is in line by our findings that P.andersonii root nodule formation is highly tolerant to exogenous nitrates.

The Parasponia-rhizobium symbiosis is considered to be relatively young when compared to legumes, and only evolved after emergence of the Paraspona lineage in the genus Trema (Yang et al., 2013; Werner et al., 2014; Behm et al., 2014). Such young symbiosis may lack sophisticated mechanisms to control its symbiotic partner, and/or may even employ a different strategy than known from legumes. Our finding that formation of fixation threads, and the nitrogen-fixing stage of intracellular rhizobium in the nodules, is most strictly controlled in presence of exogenous nitrate shows that P.andersonii employs a novel strategy to control its symbiotic partner. In this way the energy demanding process of biological nitrogen fixation is prohibited, rather than the less energetic process of nodule formation. This finding provides new insights in the evolutionary plasticity on the nitrogen fixing symbiosis trait.

\section{Materials and methods}

\section{Plant material and growth conditions and transformation}

$P$. andersonii accession WU1 was propagated in in vitro cell culture as described previously (Cao et al., 2012). Plantlets were transferred to the $14 \mathrm{~cm}$ round pots containing sterilized sand:granule mixture (v/v 1:1) supplemented with EKM medium with the required $\mathrm{KNO}_{3}$ concentration (Cao et al., 2012). Plants were inoculated with $10 \mathrm{ml}$ Bradyrhizobium elkanii WUR3 at a final optical density of $\mathrm{OD}_{600}$ : 0.15 (Op den Camp et al., 2012). Plants were grown under greenhouse conditions at $28^{\circ} \mathrm{C}, \sim 100 \% \mathrm{RH}, 12 / 12$ day/night regime and irrigated three times per week; once with nitrogen free EKM medium and two times with water. Non-inoculated plants were grown in the same condition as control. All plants were watered with EKM-medium minus $\mathrm{N}$-source, whereas $\mathrm{KNO}_{3}$ was used only once at the start of the experiment. Plant were harvested 8 weeks post inoculation ( $\mathrm{n}=10$ / treatment).

Fresh weights were determined immediately after harvesting. Nodules from each plant were removed from the root, counted and weighed. Dry weights were measured after desiccation in an oven at $60^{\circ} \mathrm{C}$ for $24 \mathrm{~h}$.

Transgenic compound $P$. andersonnii plants carrying either a PaNFP RNAi construct (Op den Camp et al., 2011) or a cameleon NupYC2.1 construct (Granqvist et al., 2015) were generated 
using Agrobacterium rhizogenes mediated transformation (Cao et al., 2012). Plants containing NupYC2.1 were kept in vitro. Calcium oscillation experiments were conducted as described by Granqvist et at 2015, PaNFP RNAi plants were grown in the sand:granule system as described above. Plants transformed with an empty vector considered as control and grown in the same condition with PaNFP RNAi knock down plants.

\section{Nitrogenase activity}

Nitrogenase activity was measured by using acetylene reduction assay (ARA) (Hardy et al., 1973). Individual roots with attached nodules were placed in $35 \mathrm{ml}$ bottle and sealed. $3.5 \mathrm{ml}$ volume of air was withdrawn with a syringe and replaced by $3.5 \mathrm{ml}$ of acetylene. After $1 \mathrm{~h}$ incubation in room temperature, $1 \mathrm{ml}$ of gas were taken from the bottle and injected into the gas chromatograph system. Ethylene production was determined by gas chromatograph (Chrompack Packard 438A equipped with Porapak N column $(110 \mathrm{~cm} \times 1.6 \mathrm{~mm}$ ID $)$ using a $\mathrm{N}_{2}$ as a carrier gas at a flow rate of $30 \mathrm{ml} / \mathrm{min}$ at $60{ }^{\circ} \mathrm{C}$.

\section{RNA extraction and Quantitative real-time polymerase chain reaction ( $q R T-P C R)$}

Total RNA was isolated from $P$. andersonii roots and treated with DNAse I using Qiagen plant RNAeasy kit (Qiagen; according to the manufacture's instruction). RNA concentration was quantified by NanoDrop spectrophotometer. cDNA was synthesized from $1 \mu \mathrm{g}$ of total RNA using an i-script cDNA synthesis kit (Bio-Rad, Hercules, USA) as described in the manufacturer's protocol. Quantitive PCR reaction was performed in triplicate using primers for PaNFP and PaACTIN1 as described previously (Op den Camp et al., 2011).

\section{Nodule structure and ultrastructure}

Nodules were embedded in Technovit 7100 according to the supplier' protocols (HeraeusKulzer, Wehrheim, Germany). Sections (4-6 $\mu \mathrm{m}$ ) were cut on a microtome (RJ2035, Leica Microsystems), stained with toluidine blue $(0.5 \%)$ and analysed by light microscopy (Leica AU5500B equipped with DFC425C Camera).

For transmission electron microscopy nodule tissue was prepared as described before (Fedorova et al., 1999). Nodules were fixed in a 3\% glutararaldehyde/4\% paraformaldehyde mix, post-fixed by $\mathrm{OsO}_{4}$ and embedded in LR Whine resin. Sections of $60 \mathrm{~nm}$ were prepared using a Leica 


\section{Chapter 4}

Ultracut microtome. Nickel grids were counterstained and examined using a JEOL JEM 2100 transmission electron microscope equipped with a Gatan US4000 4K×4K camera.

\section{Acknowledgments}

This work was supported by the European Research Council (ERC-2011-AdG294790) (MSK, EF, JV, RvV, TB, RG \& RG), the NWO-NSFC Joined Research project (846.11.005) (TB \& RG), NWO-VICI (865.13.001) (RG). 


\section{Supplemental figures}

A

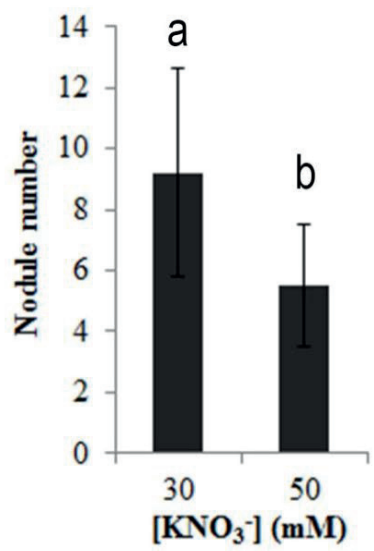

B

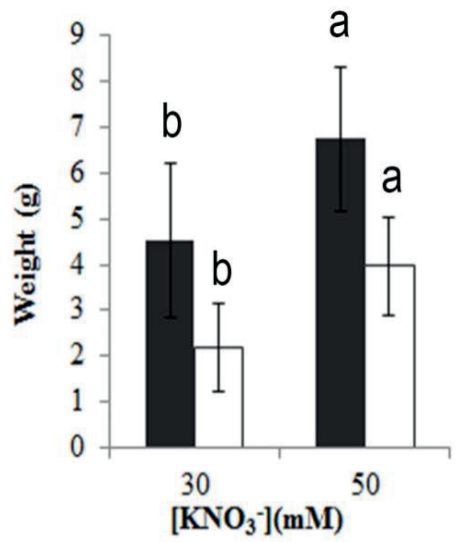

Figure S1. Effects of $30 \mathrm{mM}$ and $50 \mathrm{mM} \mathrm{KNO}_{3}$ on Parasponia andersonii inoculated with Bradyrhizobium elkanii WUR3 (eight weeks post inoculation). (A) Nodulation efficiency at different concentrations of exogenous nitrate. (B) Effect of exogenous nitrate on plant fresh weight (black bars: shoot fresh weight and white bars: root fresh weight $)(n=10)$. 


\section{Chapter 4}

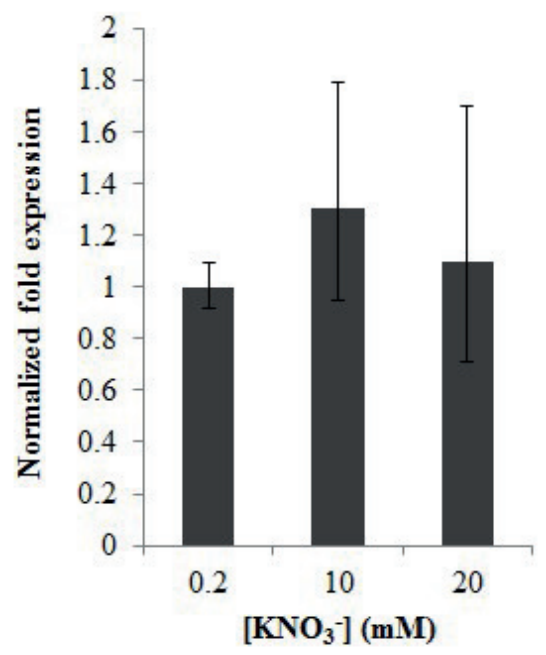

Figure S2. Relative expression of PaNFP in roots of Parasponia andersonii grown on $0.2,10$ and $20 \mathrm{mM} \mathrm{KNO}_{3}$.

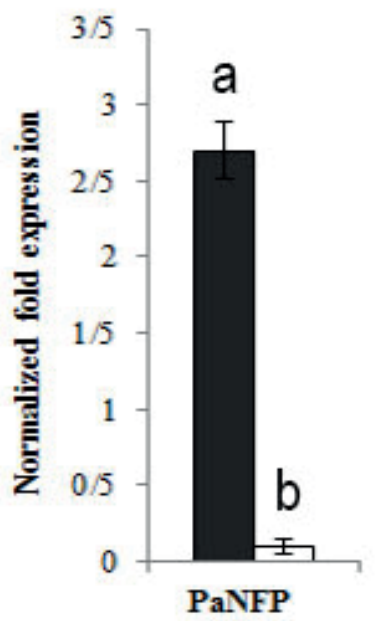

Figure S3. PaNFP expression level in non-transgenic roots (black bars) and PaNFP RNAi knockdown roots (white bars) grown at $0.2 \mathrm{mM} \mathrm{KNO}_{3}$. 


\section{References}

Barbulova A, Rogato A, D’Apuzzo E, Omrane S, Chiurazzi M. 2007. Differential effects of combined N sources on early steps of the Nod factor-dependent transduction pathway in Lotus japonicus. Molecular plant-microbe interactions 20, 994-1003.

Behm JE, Geurts R, Kiers ET. 2014. Parasponia: a novel system for studying mutualism stability. Trends in Plant Science 19, 757-763.

Broghammer a., Krusell L, Blaise M, et al. 2012. Legume receptors perceive the rhizobial lipochitin oligosaccharide signal molecules by direct binding. Proceedings of the National Academy of Sciences of the United States of America 109, 13859-13864.

Cao Q, Camp R den, Kalhor M. 2012. Efficiency of Agrobacterium rhizogenes-mediated root transformation of Parasponia and Trema is temperature dependent. Plant Growth Regulation 68, 459-465.

Carroll BJ, Mathews A. 1990. Nitrate inhibition of nodulation in legumes. In: Gresshoff PM, ed. The Molecular Biology of Symbiotic Nitrogen fixation. CRC Press, 159-180.

Doyle JJ. 2011. Phylogenetic perspectives on the origins of nodulation. Molecular plant-microbe interactions 24, 1289-95.

Faria SM De, Sutherland JM, Sprent JI. 1986. A new type of infected cell in root nodules Ofandira Spp. (Leguminosae). Plant Science 45, 143-147.

Fedorova E, Thomson R, Whitehead LF, Maudoux O, Udvardi MK, Day DA. 1999. Localization of H + -ATPases in soybean root nodules. Planta 209, 25-32.

Gavrin A, Kaiser BN, Geiger D, Tyerman SD, Wen Z, Bisseling T, Fedorova EE. 2014. Adjustment of Host Cells for Accommodation of Symbiotic Bacteria: Vacuole Defunctionalization, HOPS Suppression, and TIP1g Retargeting in Medicago. The Plant Cell 26, 3809-3822.

Geurts R, Lillo A, Bisseling T. 2012. Exploiting an ancient signalling machinery to enjoy a nitrogen fixing symbiosis. Current Opinion in Plant Biology 15, 438-443.

Granqvist E, Sun J, Camp RO Den, Pujic P, Hill L, Normand P, Morris RJ, Downie JA, Geurts R, Oldroyd GED. 2015. Bacterial-induced calcium oscillations are common to nitrogen-fixing associations of nodulating legumes and 


\section{Chapter 4}

non-legumes. New Phytol. 207, 551-558.

Hardy RWF, Burns RC, Holsten RD. 1973. Applications of the acetylene-ethylene assay for measurement of nitrogen fixation. Soil Biology and Biochemistry 5, 47-81.

Harper JE, Gibson a. H. 1984. Differential nodulation tolerance to nitrate among Legume species. Crop Science 24, 797.

Heidstra R, Geurts R, Franssen H, Spaink HP, Van Kammen A, Bisseling T. 1994. Root hair deformation activity of nodulation factors and their fate on Vicia sativa. Plant physiology 105, 787-797.

Heidstra R, Yang WC, Yalcin Y, Peck S, Emons a M, van Kammen A, Bisseling T. 1997. Ethylene provides positional information on cortical cell division but is not involved in Nod factor-induced root hair tip growth in Rhizobium-legume interaction. Development 124, 1781-1787.

Horváth B, Yeun LH, Domonkos Á, et al. 2011. Medicago truncatula IPD3 is a member of the common symbiotic signaling pathway required for rhizobial and mycorrhizal symbioses. Molecular Plant-Microbe Interactions 24, $1345-1358$.

Huss-Danell K. 1997. Actinorhizal symbiosis and their N2 fixation. New Phytologist 136, 375-405.

Kohls SJ, Baker DD. 1989. Effects of substrate nitrate concentration on symbiotic nodule formation in actinorhizal plants. Plant and Soil 118, 171-179.

Lancelle SA, Torrey JG. 1984. Early development of Rhizobium-induced root nodules of Parasponia rigida. I. Infection and early nodule initiation. Protoplasma 123, 26-37.

Lavin M, Herendeen PS, Wojciechowski MF. 2005. Evolutionary rates analysis of Leguminosae implicates a rapid diversification of lineages during the tertiary. Systematic biology 54, 575-94.

Li H-L, Wang W, Mortimer PE, Li R-Q, Li D-Z, Hyde KD, Xu J-C, Soltis DE, Chen Z-D. 2015. Large-scale phylogenetic analyses reveal multiple gains of actinorhizal nitrogen-fixing symbioses in angiosperms associated with climate change. Scientific Reports 5, 14023.

Limpens E, Mirabella R, Fedorova E, Franken C, Franssen H, Bisseling T, Geurts R. 2005. Formation of organellelike N2-fixing symbiosomes in legume root nodules is controlled by DMI2. Proceedings of the National Academy of Sciences of the United States of America 102, 10375-10380. 
Marsh JF, Rakocevic A, Mitra RM, Brocard L, Sun J, Eschstruth A, Long SR, Schultze M, Ratet P, Oldroyd GED. 2007. Medicago truncatula NIN is essential for rhizobial-independent nodule organogenesis induced by autoactive Calcium/Calmodulin-Dependent Protein Kinase. Plant physiology 144, 324-335.

Marvel DJ, Torrey JG, Ausubel FM. 1987. Rhizobium symbiotic genes required for nodulation of legume and nonlegume hosts. Proceedings of the National Academy of Sciences of the United States of America 84, 1319-1323.

Miri M, Janakirama P, Held M, Ross L, Szczyglowski K. 2016. Into the Root: How Cytokinin Controls Rhizobial Infection. Trends in Plant Science xx, 1-9.

Moling S, Pietraszewska-Bogiel A, Postma M, Fedorova E, Hink M a., Limpens E, Gadella TWJ, Bisseling T. 2014. Nod factor receptors form heteromeric complexes and are essential for intracellular infection in Medicago nodules. The Plant Cell 26, 4188-4199.

Naisbitt T, James EK, Sprent JJI. 1992. The evolutionary significance of the legume genus Chamaecrista, as determined by nodule structure. New Phytologist 122, 487-492.

Oldroyd GED. 2013. Speak, friend, and enter: signalling systems that promote beneficial symbiotic associations in plants. Nature reviews. Microbiology 11, 252-63.

Op den Camp RHM, Polone E, Fedorova E, Roelofsen W, Squartini A, Camp HJMO Den, Bisseling T, Geurts R. 2012. Nonlegume Parasponia andersonii deploys a Broad Rhizobium Host Range Strategy Resulting in Largely Variable Symbiotic Effectiveness. Molecular Plant-Microbe Interactions 25, 954-963.

Op den Camp R, Streng A, De Mita S, et al. 2011. LysM-type mycorrhizal receptor recruited for rhizobium symbiosis in nonlegume Parasponia. Science (New York, N.Y.) 331, 909-912.

Ovchinnikova E, Journet E-P, Chabaud M, et al. 2011. IPD3 controls the formation of nitrogen-fixing symbiosomes in pea and Medicago Spp. Molecular plant-microbe interactions 24, 1333-44.

Pawlowski K, Demchenko KN. 2012. The diversity of actinorhizal symbiosis. Protoplasma 249, 967-979.

Price GD, Mohapatra SS, Gresshoff PM. 1984. Structure of nodules formed by Rhizobium strain ANU289 in the nonlegume Parasponia and the legume Siratro (Macroptilium atropurpureum). Botanical Gazette 145, 444.

Sagan M, Morandi D, Tarenghi E, Duc G. 1995. Selection of nodulation and mycorrhizal mutants in the model plant 


\section{Chapter 4}

Medicago truncatula (Gaertn.) after gamma-ray mutagenesis. Plant Science 111, 63-71.

Schauser L, Roussis A, Stiller J, Stougaard J. 1999. A plant regulator controlling development of symbiotic root nodules. Nature 402, 191-195.

Sprent JI. 2008. 60Ma of legume nodulation. What's new? What's changing? Journal of experimental botany 59, $1081-1084$.

Streeter J. 1988. Inhibition of legume nodule formation and N2 fixation by nitrate. Critical Reviews in Plant Sciences 7, 1-23.

Streng A, op den Camp R, Bisseling T, Geurts R. 2011. Evolutionary origin of rhizobium Nod factor signaling. Plant Signaling \& Behavior 6, 1510-1514.

Werner GDA, Cornwell WK, Sprent JI, Kattge J, Kiers ET, Werner. 2014. A single evolutionary innovation drives the deep evolution of symbiotic N2-fixation in angiosperms. Nature communications 5, 4087.

Willems A. 2006. The taxonomy of rhizobia: an overview. Plant and Soil 287, 3-14.

Yang M, Velzen R Van, Bakker F, Sattarian A. 2013. Molecular phylogenetics and character evolution of Cannabaceae. Taxon 62, 473-485. 


\section{Chapter 5}

Symbiotic Functioning of the GRAS-TYPE Transcriptional Regulators NSP1 and NSP2 is Conserved in the Non-legume Parasponia andersonii

Maryam Seifi Kalhor, Ton Bisseling and René Geurts. 


\section{Chapter 5}

\section{Abstract}

GRAS proteins are plant-specific transcriptional regulators that play critical and diverse roles in plant development and signaling. Two genes of this family namely, NODULATION SIGNALING PATHWAY1 (NSP1) and NSP2, have been identified in legumes to function in symbioses with rhizobium and endomycorrhizal fungi. Knockout mutations in either gene in pea (Pisum staivum), Medicago truncatula and Lotus japonicus block rhizobium induced root nodule formation and bacterial infection. In contrast, the mycorrhizal phenotypes of legume nspl and nsp2 knockout mutants are relatively weak and only observed in quantitative assays. This suggests that in legumes $N S P 1$ and NSP2 have been recruited to commit an essential function in rhizobium symbiosis, whereas their functioning in endomycorrhizal symbiosis is partially redundant. We investigated the symbiotic functions of NSP1 and NSP2 in Parasponia, the only known non-legume lineage that acquired the rhizobium symbiosis by convergent evolution. RNAi mediated knockdown levels of Parasponia andersonii PanNSP1 or PanNSP2 correlated with reduced rhizobium nodulation efficiencies, including plants that were deprived from any nodule-like structure. However, knockdown of PanNSP1 and PanNSP2 only partially affected root mycorrhization. Only plants with highest knockdown levels showed a decrease in arbuscule formation. This suggests that NSP1 and NSP2 also in P. andersonii root nodule formation commit an essential function, whereas their functioning in endomycorrhization is less critical. Taken-together, we conclude that symbiotic functioning of NSP1 and NSP2 is conserved in legumes and in the non-legume Parasponia.

Keywords: GRAS proteins, endomycorrhizal fungi, Parasponia andersonii, PanNSP1, PanNSP2. 


\section{Introduction}

Legume root nodules provide a niche to symbiotic rhizobium bacteria to convert atmospheric nitrogen into ammonia; a form that is accessible for plants. As this symbiosis is important in agriculture, it is intensively studied. Basic research provided insights in the underlying molecular mechanisms that drive the mutualistic relation between plant and microbe. Most prominently, it was found that signaling networks as well as cellular processes that are essential for root nodule formation, are co-opted from the much older -and more widespread- arbuscular mycorrhizal (AM) symbiosis (Parniske, 2008). Besides legumes, which form a large taxonomic family encompassing tens of thousands of species, a similar nitrogen fixing rhizobium root nodule symbiosis occurs also on five tropical tree species of the genus Parasponia (Cannabaceae) (Behm et al., 2014). As the symbiosis trait in legumes and Parasponia evolved independently (Doyle, 2011), a comparative analysis may provide unique insights in the genetic constraints of this symbiosis.

Root nodule formation and rhizobium infection have been genetically investigated in the legume models Medicago truncatula and Lotus japonicus. This revealed that the rhizobium symbiosis has co-opted parts of the genetic network that plants used to establish an endomycorrhizal symbiosis. Genes that have a dual role in rhizobium and endomycorrhizal symbiosis encode a plasma-membrane localized LECTIN NUCLEOTIDE PHOSPHOHYDROLASE (LjLNP) (Roberts et al., 2013), a protein complex of the LRR-type receptor kinase LjSYMRK/MtDMI2, a key enzyme of the mevalonate biosynthetic pathway (3-Hydroxy-3-Methylglutaryl CoA Reductase 1; MtHMGR1), a DNA-binding protein LjSIP1(Wang et al., 2013; Endre et al., 2002; Stracke et al., 2002; Sun et al., 2015), nuclear envelope localized cation channels LjCASTOR and LjPOLLUX/MtDMI1(Ané et al., 2004; Imaizumi-Anraku et al., 2005), a nuclear localized Calcium and Calmodulin-dependent protein kinase (CCaMK) (Ané et al., 2004) and the transcription factors LjCYCLOPS/MtIPD3, NSP1 and NSP2 (Yano et al., 2008; Maillet et al., 2011; Horváth et al., 2011; Delaux et al., 2013). By studying the epistatic relationships among most of these genes a genetic network, stretching from the receptors down to the transcriptional regulators has been revealed (Geurts et al., 2016). As this network controls both rhizobium as well as endomycorrhizal symbiosis it is named the common symbiosis signaling network. 


\section{Chapter 5}

Interestingly, the strengths of the phenotypes of knockout mutants of the genes in this network differ between both symbioses. This is most prominent for the transcriptional regulators NSP1 and NSP2. A knockout mutation in either gene in L. japonicus, M. truncatula or pea (Pisum sativum), blocks rhizobium infection and root nodule formation (Kaló et al., 2005; Smit et al., 2005; Heckmann et al., 2011; Shtark et al., 2016). In contrast, endomycorrhization phenotypes of $n s p 1$ and $n s p 2$ mutants in any of these species are relatively weak. Reduced colonization efficiencies and arbuscule formation could only be detected by sensitive quantitative assays in which a reduced number of mycorrhizal spores was used as inoculum (Maillet et al., 2011; Lauressergues et al., 2012; Delaux et al., 2013; Takeda et al., 2013; Shtark et al., 2016). This let us to speculate that in legumes NSP1 and NSP2 are evolved to commit an essential function in rhizobium symbiosis, whereas their functioning in endomycorrhizal symbiosis is partially redundant.

NSP1 and NSP2 belong to the plant specific class of GRAS transcriptional regulators. In plants GRAS proteins are highly conserved and can be divided into several clades, each with distinct conserved domains and functions (Tian et al., 2004; Lee et al., 2008; Engstrom, 2011; Liu and Widmer, 2014) . NSP1 and NSP2 belong to two different clades; namely SHORT ROOT (SHR) and HAIRY MERISTEM (HAM), respectively (Kaló et al., 2005; Smit et al., 2007). Generally, GRAS proteins commit roles in shoot and root development, Gibberellin (GA3) signaling, phytochrome A signaling, abiotic stress response or symbioses. In latter case, besides NSP1 and NSP2, at least two additional symbiotic GRAS proteins have been identified that function in endomycorrhizal symbiosis in legumes; LjRAD1 and LjRAM1/MtRAM1(Gobbato, 2015; Zhang et al., 2015). Furthermore, at least 6 GRAS proteins are transcriptionally induced in $M$. truncatula roots upon mycorrhization (Hogekamp et al., 2011). Several of these symbiotic GRAS proteins showed to form heteromeric protein complexes, allowing an additional level of regulation. For example, it was found that MtNSP2 interacts with MtRAM1 and MtNSP1 to regulate the expression of the glycerol-3-phosphate acyl transferase MtRAM2, a gene essential for arbuscule formation by endomycorrhizal fungi (Gobbato et al., 2012).

Studies in M. truncatula revealed that MtNSP1 and MtNSP2 control expression of several genes in a symbiotic context; including MtENOD11 and MtDWARF27 (MtD27) (Liu et al., 2011). $M t D 27$ encodes a plastid localized carotenoid isomerase, which functions in the strigolactone biosynthetic pathway. Strigolactone biosynthesis is under the control of nutrient sensing 
mechanisms, especially phosphate deficiency stress (Yoneyama et al., 2012). Root-exuded strigolactones can activate AM-fungi, thereby promoting endomycorrization of the root (Akiyama et al., 2005; Besserer et al., 2006). As strigolactone biosynthesis in Medicago is -at least in part- controlled by transcriptional regulation of MtD27 in a MtNSP1 and MtNSP2dependent manner (Liu et al., 2011; van Zeijl et al., 2015), it may explain the reduction in root colonization by endomycorrhizal fungi in a Mtnsp1Mt/nsp2 knockout mutant.

Studies in Parasponia have shown that evolution of rhizobium symbiosis is guided by genetic constraints (Geurts et al., 2016). Similar to legumes the Parasponia-rhizobium symbiosis is founded on perception of rhizobium secreted lipo-chitooligosaccharide (LCO) signal molecules (Marvel et al., 1987; den Camp et al., 2011). In both lineages these LCOs are perceived by orthologous LysM-type receptors that activate the common symbiosis signaling network (Streng et al., 2011). In Parasponia as demonstrated by ectopic expression of an autoactive allele of CCaMK, which leads to formation of nodule-like structures in absence of rhizobium (Op den Camp et al., 2011).

We questioned whether NSP1 and NSP2 fulfil a symbiotic function in Parasponia. We identified single putative orthologs of NSP1 and NSP2 in the draft genome sequences of Parasponia andersonii and Trema ortientalis. By conducting RNAi in P. andersonii roots we demonstrate that PanNSP1 and PanNSP2 are essential for rhizobium root nodule formation. However, knockdown of $P$. andersonii PanNSP1 and PanNSP2 only partially affected mycorrhization. This suggests that NSP1 and NSP2 in P. andersonii root nodule formation commit an essential function, whereas their functioning in endomycorrhization is less critical. Taken together, we conclude that symbiotic functioning of NSP1 and NSP2 is conserved in legumes and the nonlegume Parasponia.

\section{Results}

\section{Identification of Parasponia andersonii NSP1 and NSP2}

The GRAS protein family is largely conserved in plants and represents 12 distinct clades named according to a representative protein of Arabidopsis thaliana (Arabidopsis) (Liu and Widmer, 2014). NSP1 and NSP2 belong to two different clades; SHORT ROOT (SHR) and HAIRY 


\section{Chapter 5}

MERISTEM (HAM), respectively (Smit et al., 2005; Kaló et al., 2005; Engstrom, 2011). To identify the Parasponia NSP1 and NSP2 orthologous genes, the draft genome sequence assemblies of $P$. andersonii and T. orientalis were mined using the Arabidopsis members of both subclades as query. To create a robust phylogenetic structure similar searches were conducted in M. truncatula and Glycine max genome annotations Mt4.0v1 and Wm82.a2.v1.

The SHR clade contains 4 Arabidopsis genes. Besides AtSHR, these are AtBLS1 (BRASSINOSTEROID, LIGHT AND SUGAR 1), At5G67411 and AtSCL29 (SCARECROW-LIKE 29). The latter represents the putative ortholog of legume NSP1. Searching the P. andersonii and $T$. orientalis genome resulted in the identification of 5 orthologous genes, whereas for $M$. turncatula and G. max genes 5 and 12 genes could be identified, respectively. Phylogenetic analysis revealed 5 orthology groups (OGs) each containing at least one gene of $P$. andersonii, $T$. orientalis, G. $\max$ and M. truncatula. Arabidopsis genes only grouped in 3 OGs including AtSCL29, which represents the NSP1 OG. (Figure 1B). The putative NSP1 orthologs of $P$. andersonii and T. orientalis were named accordingly PanNSP1 and TorNSP1.

The HAM clade includes 5 Arabidopsis genes; AtHAMI to AtHAM4 and AtSCL26, respectively. The latter represents the putative ortholog of legume NSP2 (Kaló et al., 2005). Blast searches in P. andersonii, T. orientalis, M. truncatula and $G$. max and subsequent phylogenetic reconstruction revealed 5 orthology groups, two of which were devoid of an Arabidopsis orthologous gene. MtNSP2 clustered with a single $P$. andersonii, T. orientalis and Arabidopsis gene, but with four G. $\max$ and an additional M. truncatula paralog. The phylogenetic structure of the NSP2 OG suggests that M. truncatula and G. max share the duplication event (Figure 1A). The $P$. andersonii and $T$. orientalis genes were named accordingly PanNSP2 and TorNSP 2 . Pairwise alignment of the PanNSP1 and PanNSP2 proteins to their orthologs in T. orientalis showed that both gene orthologs are $\sim 94 \%$ identical and shared a $65 \%$ (NSP1) and $59 \%$ (NSP2) identify with the $M$. truncatula proteins. Furthermore, the 5 conserved GRAS domains - leucine heptad repeat I (LHR-I), VHIID, LHR-II, PFYRE and SAW- were present in the Parasponia proteins. Taken together, this suggests that PanNSP1 and PanNSP2 represent functional GRAStype transcriptional regulators. 


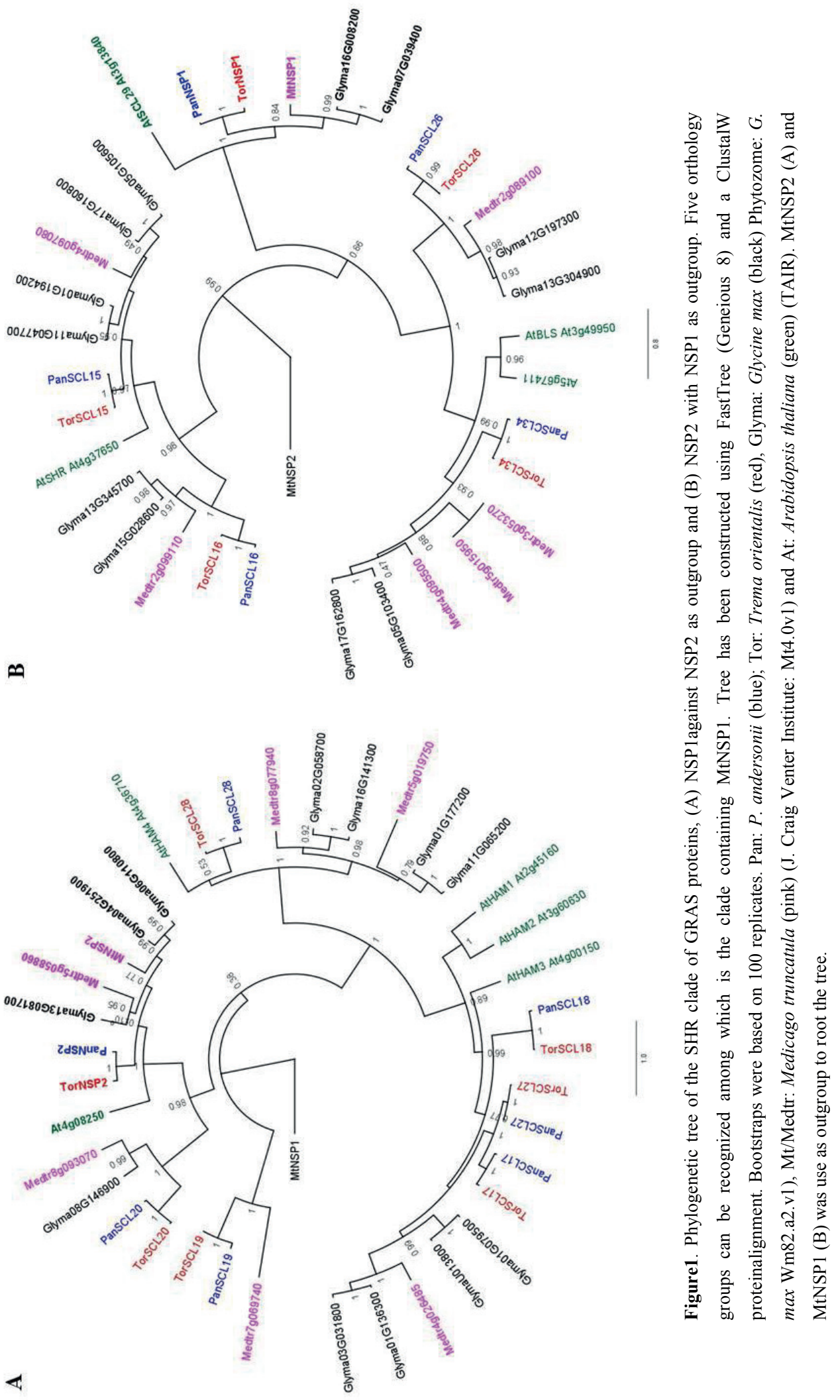




\section{Chapter 5}

\section{Parasponia NSP1 and NSP2 are essential for root nodulation}

First we determined whether PanNSP1 and PanNPS2 are expressed in roots of P. andersonii grown under susceptible conditions for root nodulation (EKM medium supplemented with 0.2 $\mathrm{mM} \mathrm{KNO}{ }_{3}$ ). RNA was isolated from young growing roots and subsequently used as template in a qRT-PCR experiment to determine relative expression of PanNSP1 and PanNPS2 genes. This revealed that both genes are expressed in the root at comparable levels.

Next, we investigated whether PanNSP1 and PanNSP2 are essential for root nodule formation. To do so, gene specific RNAi constructs were made in the binary vector pK7GWIGW2 (II)-RR that includes a DsRED1 reporter as non-destructive selection marker. These PanNSP1 and PanNSP2 RNAi vectors were introduced in P. andersonii roots using Agrobacterium rhizogenesmediated transformation. Plants with transgenic roots were selected based on red fluorescence. Of these plants non-fluorescent (non-transgenic) roots were removed prior transfer to pots. For each construct at least 5 RNAi knockdown plants were selected as well as a similar number of control plants (transformed with an empty vector construct) and subsequently inoculated with Bradyrhizobium elkanii WUR3. Eight weeks post inoculation roots were harvested to determine the nodulation efficiency of transgenic (red fluorescent) roots. Roots of control plants transformed with empty vector- harbored $\sim 15$ nodules (in average). In contrast, nodule number on PanNSP1 and PanNSP2 knockdown roots varied, ranging between 0 and 15 nodules/plant (Figure 3A, B; Table 1) 
A

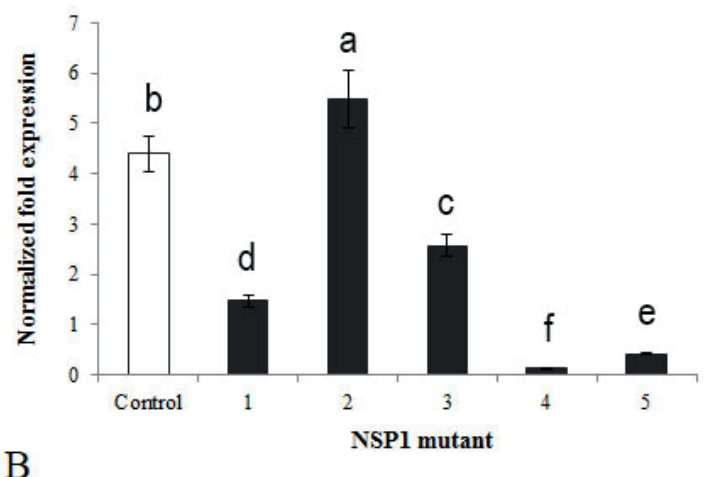

B

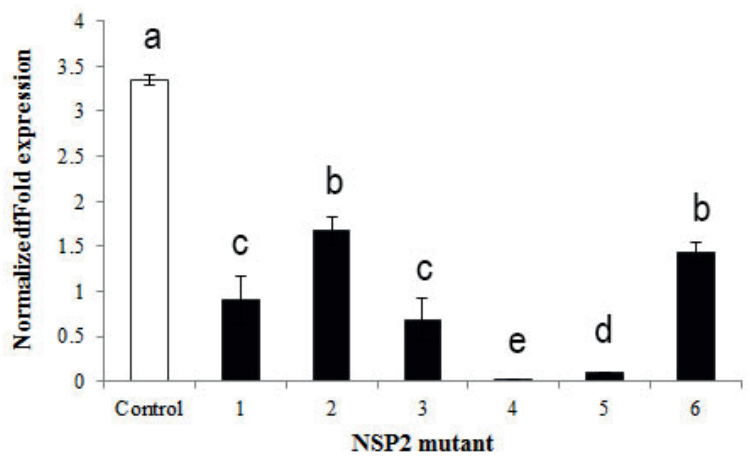

Figure2. PanNSP expression level in roots of mutants and control plants (harbouring empty vector) 8 weeks after inoculation with Bradyrhizobium elkanii (WUR3). (A) Level of transcription of PanNSP1 in Parasponia RNAi knocked down roots (black bars) versus control root (transformed with empty vector, white bars). (B) Quantification of PanNSP2 in Parasponia knocked down roots (black bars) versus control roots (transformed with empty vector, white bars). Quantifications were normalized using stable expressed reference gene PaACTIN. Bars represent SD of three technical repeats.

To determine whether the reduction in nodule numbers correlated with the knock down levels of PanNSP1 and PanNSP2 expression, RNA was isolated from individual roots and expression level of both genes was determined by qRT-PCR. Correlation was found between level of knockdown and nodulation efficiency (Figure 3A, B). These finding demonstrate that PanNSP1 and PanNSP2 are essential for root nodule formation in P. andersonii. 


\section{Chapter 5}

A

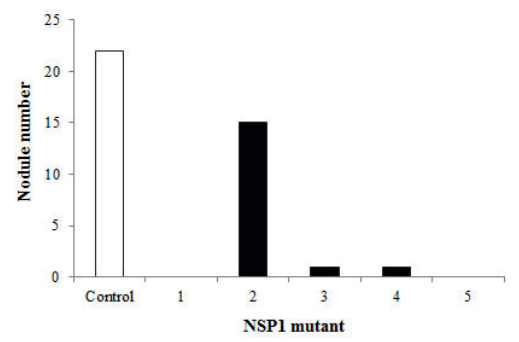

B

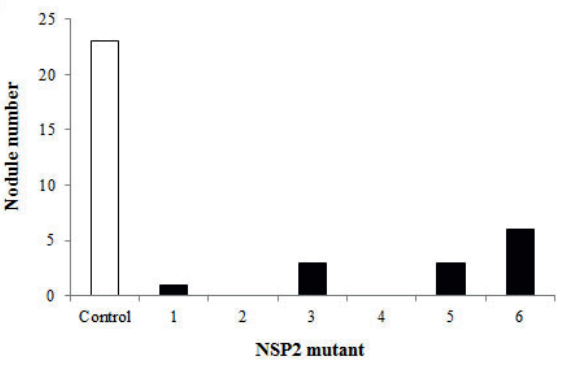

Figure 3. Nodule number in PaNSP mutants. (A) Reduced nodule number in PanNSP1 RNAi knocked down mutants (black bars) compare with control plants (withe bars). (B) Reduced nodule number in PanNSP2 RNAi knocked down mutants (black bars) compared with control plants (withe bars). Nodules counted 8 weeks after inoculation with Bradyrhizobium elkanii (WUR3).

Partial knock down of PanNSP1 (e.g. plant 2 and 3) or PanNSP2 (e.g. plant 3, 5 and 6) does not lead to a complete block of nodulation. Previous studies in Lotus suggest that LjNSP1 and LjNSP2 may have a function also in maintenance of symbiotic rhizobium in the root nodule (Heckmann et al., 2006). To investigate whether PanNSP1 and/or PanNSP2 may have a similar function in P. andersonii we sectioned nodules formed on (partial) PanNSP1 or PanNSP2 knock down roots. In total we sectioned 5 PanNSP1 RNAi and 8 PanNSP2 RNAi nodules. All these nodules displayed a normal developmental phenotype, including intracellular infection (Figure 4). This suggests that PanNSP1 and PanNSP2 are essential in symbiotic signaling, but if a signaling threshold is achieved, nodule formation proceeds normally.

Table 1. Rate of infected plants in the wild type and nsp 1, nsp 2 mutants.

\begin{tabular}{|c|c|c|c|c|c|c|}
\hline & \multicolumn{3}{|l|}{ B.elkanii } & \multicolumn{3}{|c|}{ R.irregularis } \\
\hline & $\begin{array}{l}\text { Number of } \\
\text { inoculated } \\
\text { plants }\end{array}$ & $\begin{array}{l}\text { Number of } \\
\text { nodulated } \\
\text { plants }\end{array}$ & Ratio & $\begin{array}{l}\text { Number of } \\
\text { inoculated } \\
\text { plants }\end{array}$ & $\begin{array}{l}\text { Number of } \\
\text { mycorrhized } \\
\text { plants }\end{array}$ & Ratio \\
\hline Wild type & 10 & 10 & 1.00 & 6 & 6 & 1.00 \\
\hline Transgenic Control & 8 & 8 & 1.00 & 8 & 8 & 1.00 \\
\hline NSP1 & 15 & 7 & 0.46 & 10 & 10 & 1.00 \\
\hline NSP2 & 15 & 12 & 0.8 & 11 & 11 & 1.00 \\
\hline
\end{tabular}



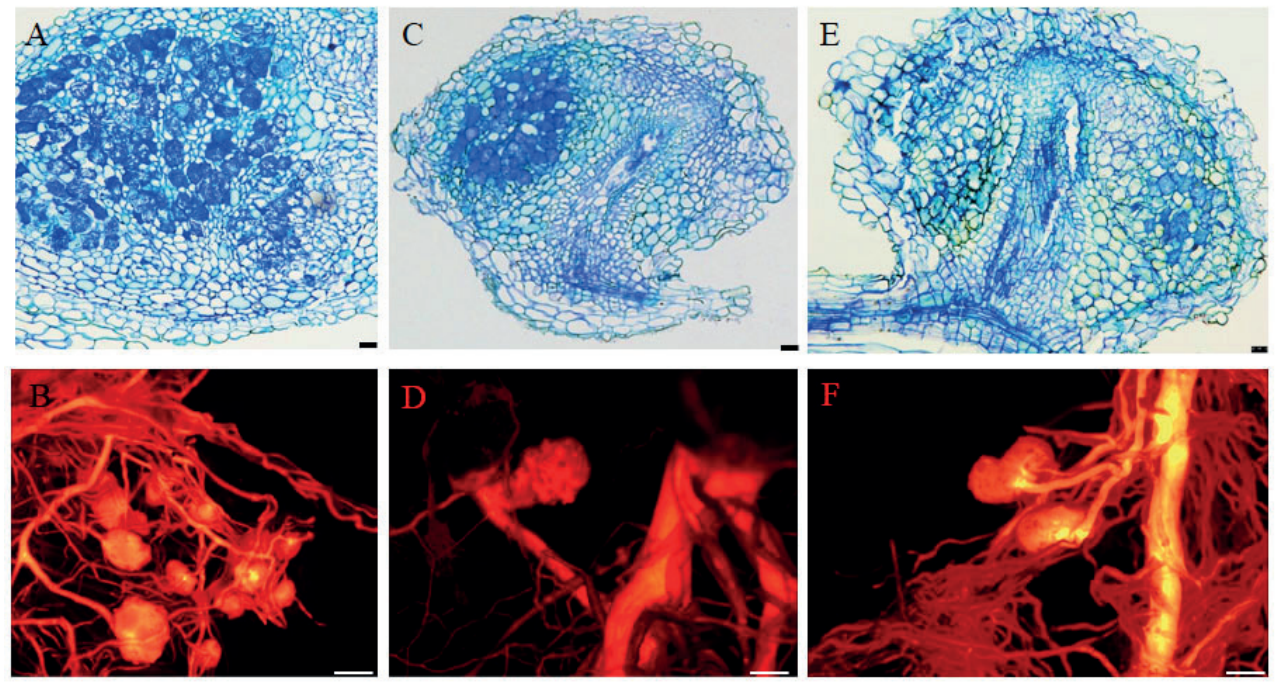

Figure 4. Parasponia nodules 8 weeks after inoculation with Bradyrhizobium elkanii (WUR3). (A) $4 \mu \mathrm{m}$ thin resin embedded section of control nodule (transformed with empty vector). (B) Transgenic nodule selected based on red fluorescence due to DsRED1 expression. (C) Longitudinal section of nodule originated from PanNSP1 knocked down root. (D) Transgenic nodule selected based on red fluorescence due to DsRED1 expression. (E) Longitudinal section of nodule grown on PanNSP2 knocked down root. (F) Transgenic nodule selected based on red fluorescence due to DsRED1 expression.

\section{Parasponia PanNSP1 and PanNSP2 are involved in endomycorrhization}

In order to determine whether PanNSP1 and/or PanNSP2 are involved in establishment of AM symbiosis, $P$. andersonii plants with knockdown and control roots were generated and grown in pots containing sterilized sand-granule (1:1) mixture as substrate. Plants were watered 3 times a week, once with Hoagland's solution (Hoagland, 1950) $\left(0 \mu \mathrm{M} \mathrm{PO}_{4}\right)$ and twice with water. For each construct 15 RNAi knockdown plants were selected as well as a similar number of control plants (transformed with an empty vector construct) and subsequently inoculated with Rhizophagus irregularis. The percentage of root colonization was examined 4 weeks post inoculation. These studies revealed that PanNSP1 and PanNSP2 knockdown roots were all mycorrhized (Table 1). However, the fraction of PanNSP1 RNAi root segments that were mycorrhized was significantly lower when compared to empty vector control roots or PanNSP2 


\section{Chapter 5}

RNAi roots (Figure 5A). Next, we determined the frequency of arbuscule formation in the infected root segments. This revealed that the number of arbuscules present in both -PanNSPI and PanNSP2- knockdown roots is significantly lower when compared to wild type control roots (Figure 5B). Taken together, these results indicate that both GRAS-type regulators are involved in -though not essential for- endomycorrhizal symbiosis.
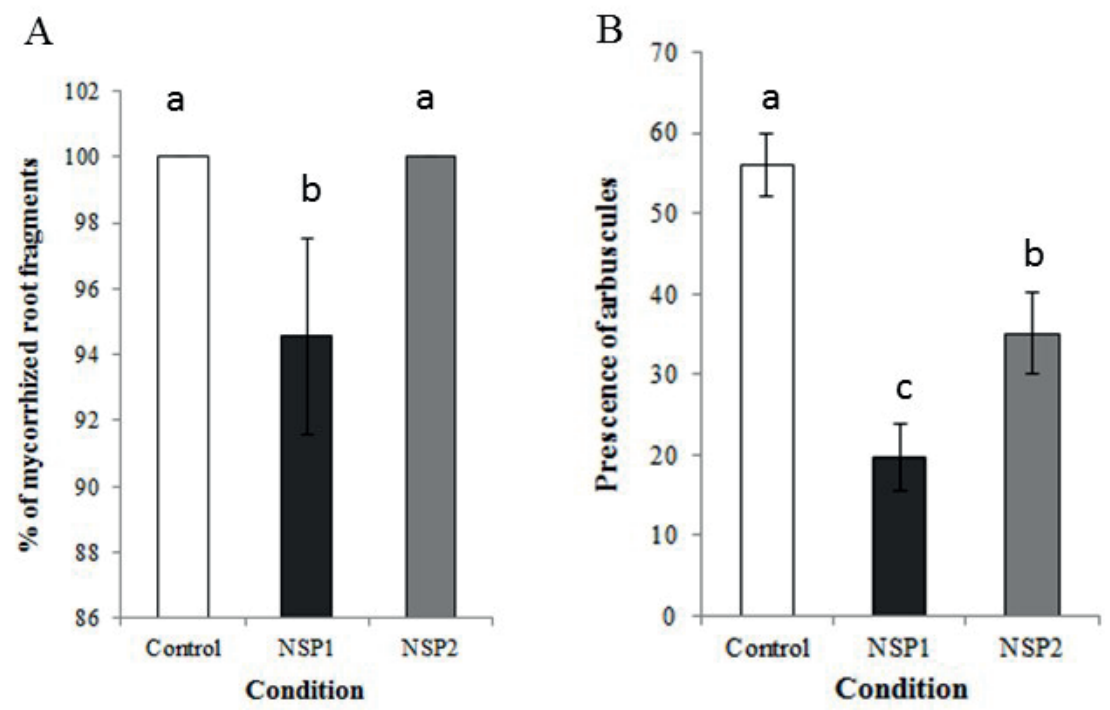

Figure 5. Analysis of Parasponia roots inoculated with $R$. iregularis.four weeks after inoculation (A) Percentage of mycorrhized root fragments in PanNSP1 and PanNSP2 RNAi knocked down mutants compare with control plants (transformed with empty vector). (B) Reduced level of arbuscul formation in mycorrhized fragments of NSP1 and NSP2 RNAi knocked down mutants compare with control plants (transformed with empty vector).

\section{Discussion}

In several legumes, it has been identified that the GRAS-type transcriptional regulators NSP1 and NSP2 are essential for rhizobium root nodule formation (Kaló et al., 2005; Smit et al., 2005; Heckmann et al., 2006; Murakami et al., 2007). Both proteins also play a promoting role in endomycorrhizal infection (Maillet et al., 2011; Delaux et al., 2013; Murakami et al., 2013). Our studies revealed a very similar role of NSP1 and NSP2 in the non-legume P. andersonii. Complete knockdown of PanNSP1 or PanNSP2 expression blocks root nodule formation, 
whereas mycorrhizal infection -although with a reduced efficiency- still occurs. As legumes and Parasponia gained the nodulation trait by convergent evolution, we conclude that recruitment of $N S P 1$ and NSP2 in rhizobium root nodule formation is under tight evolutionary constraints.

We studied the phenotype on individual knockdown roots. In case of knockdown levels of $>80 \%$ nodulation was almost completely inhibited. However, occasionally a single nodule was formed 8 weeks post inoculation. Based on the cytoarchitecture of these PanNSP1 and PanNSP2 RNAi nodules, we argue that these nodules most likely are functional. This finding is in line with phenotypes of L. japonicus Ljnsp1 and Ljnsp2 knock out mutants, where also occasionally functional nodules were found (Heckmann et al., 2006; Murakami et al., 2007). Such leaky phenotype is generally explained by gene redundancy scenario. Alternatively, both NSP proteins do not represent a core element in the rhizobium LCO signaling pathway, but rather facilitate dymbiotic signaling. This latter hypothesis is supported by genetic dissection studies of the rhizobium LCO induced signaling pathway in L. japonicus, which suggest that LjNSP1/LjNSP2 control several steps in the symbiosis signaling pathway. For example, LjNSP1 and LjNSP2 are required for rhizobium induced LjNIN expression (Smit et al., 2007) a gene encoding a transcription factor that is essential as well as sufficient for root nodule formation. However, LjNIN induced nodule formation requires LjNSPI and LjNSP2, indicating that both GRAS proteins function upstream as well as downstream of LjNIN (Schauser et al., 1999; Marsh et al., 2007; Soyano et al., 2013; Singh et al., 2014; Lin et al., 2014; Vernié et al., 2015). A facilitator function may explain such phenotype. A facilitator function of NSP1/NSP2 is also in line with the recent findings that both proteins control the carotenoid biosynthesis pathway (Liu et al., 2011; van Zeijl et al., 2015). Therefore we hypothesize that NSP1 and NSP2 are essential to create a physiological condition that facilitates LCO induced signaling and subsequent root nodule formation.

The finding that NSP proteins play a more prominent role in rhizobium root nodule formation that in endomycorrhization in independent lineages raises questions concerning the evolutionary trajectory of these proteins. Phylogenetic studies revealed that symbiotic NSP1 and NSP2 genes in legumes and Parasponia did not experience lineage specific duplication events. Furthermore, trans-complementation studies of L. japonicus Ljnsp1 and Ljnsp2 mutants with putative orthologs of Nicotiana benthamiana and/or rice (Oryza sativum) revealed that both proteins are 


\section{Chapter 5}

functionally conserved in higher plants (Heckmann et al., 2006; Yokota et al., 2010). This leaves only two possible scenarios concerning the evolutionary trajectory of both genes; adaptations in cis regulatory elements, or alternatively no symbiosis specific adaptations. Transcription levels and regulation in response to rhizobium LCOs was found to be important to control nodulation efficiency (Murakami et al., 2013). Also it was noted that expression of OsNSP2 in rice is relatively low (Liu et al., 2011). However, since a direct comparison of functional promoters of NSP1 and/or NSP2 of nodulating and non-nodulating species has not been conducted, it remains elusive whether specific adaptations in cis regulatory elements of either of both genes were an essential step in evolution of root nodules.

The Parasponia lineage in the Cannabaceae family represents an independent event of evolution of nitrogen fixing symbiosis with rhizobium. When compared to legumes, the Parasponiarhizobium symbiosis is relatively young, and subsequently less advanced (Behm et al., 2014). We used Parasponia to get insight in the evolutionary constraints in rhizobium root nodule formation and identified the GRAS proteins PanNSP1 and PanNSP2 as key proteins in this process. In a previous study we identified the LysM-type receptor PanNFP and PanCCaMK as being essential for root nodule formation in Parasponia (den Camp et al., 2011). Taken together, it suggests that evolution of rhizobium symbiosis is under severe genetic constraints. Such information is of considerable importance for any attempts to engineer a nitrogen fixing symbiosis on non-legume species other than Parasponia.

\section{Materials and methods}

\section{In vitro Micro-propagation of Parasponia}

Parasponia andersonii buds were surface sterilized in 4\% hypochlorite (commercial bleach) for 10 min and subsequently washed six times with sterile MQ water. Sterilized $P$. andersonii axillary buds were placed into propagation medium $(20 \mathrm{~g} / 1$ sucrose, $2.4 \mathrm{~g} / \mathrm{L}$ McCown Woody Plant Medium (WPM) including vitamins (Duchefa Biochemie, Haarlem, The Netherlands; WPM) (Lloyd and McCown, 1980), $1.0 \mathrm{mg} / \mathrm{L}$ 6-Benzylaminopurine, $0.1 \mathrm{mg} / \mathrm{L}$ Indole-3-butyric acid, 0.8\% Daichin agar, $\mathrm{pH} \mathrm{5.8,)} \mathrm{in} \varnothing 10 \mathrm{~cm}$ round petri dishes. Plates were kept in an Elbanton growth cabinet at $28^{\circ} \mathrm{C}$ with a $16 / 8 \mathrm{~h}$ day/night regime. After four weeks shoots emerged from the axillary bud calluses. Shoots of $>1.0 \mathrm{~cm}$ in length were cut and transferred to rooting medium 
$(10 \mathrm{~g} / \mathrm{L}$ sucrose, $2.4 \mathrm{~g} / \mathrm{L}$ WPM $+1.0 \mathrm{mg} / \mathrm{L}$ indole-3-butyric acid, $0.1 \mathrm{mg} / \mathrm{L}$ 1-naphthaleneacetic acid, pH5.8,) in round $\varnothing 10 \mathrm{~cm}$ Petri dishes. These plates were covered by aluminum foil (for root induction) and were kept in the same growth cabinet at $28^{\circ} \mathrm{C}$ for two weeks. Rooted shoots were kept on hormone free medium (20 g/L sucrose, $2.4 \mathrm{~g} / \mathrm{L}$ WPM, 0.8\% Daichin agar, pH 5.8,) for three weeks before transferring to the pots in greenhouse.

\section{Constructs}

The PanNSP1 and PanNSP2 RNAi constructs were made by cloning of 308 and 393 bp in pENTR-D-topo vector (PanNSP1F: TAAGCGAGAACAACATT, PanNSP1R: TTACTGTCATACTTTCTC; PanNSP2F: TTAACTATTTTGTCTTTC, PanNSP2R: TTCCTTATCTCCCTGGACA). Sequence hairpin construct was made by recombination of the amplified regions into the binary vector pK7GWIWG2 (II)-RR containing DsRED1 as selectable marker.

\section{Rhizogenes Transformation}

Agrobacterium rhizogenes, strain MSU440, containing the appropriate binary plasmid (all containing DsRED1 as selectable marker) was applied on the cut site of shoots with $\sim 1 \mathrm{~cm}$ length. Two more wounds were produced with a needle dipped in A. rhizogenes. Inoculated plants were placed in line at EKM medium plates and half covered with sterile filter paper. The plates were sealed and positioned vertically at $21^{\circ} \mathrm{C}(16 / 8 \mathrm{~h}$ light/darkness). After one week the shoots were transferred to emergence medium in Daichin agar $(0.9 \% \mathrm{w} / \mathrm{v}$, Duchefa) containing $300 \mu \mathrm{g} / \mathrm{ml}$ Cefotaxime (Duchefa) and covered by a (half-) filter paper. Plants were grown for one week at $21^{\circ} \mathrm{C}$, subsequently the filter paper was removed and the plates were placed vertically into a $28^{\circ} \mathrm{C}$ growth cabinet for $7-15$ days. In this period new roots are formed that are cotransformed with the T-DNA of the binary vector. Co-transformed roots were selected based on red fluorescence. Subsequently, plantlets with transgenic roots were transferred to bigger plates containing McCown Woody Plant Medium with vitamins (Duchefa) and $300 \mu \mathrm{g} / \mathrm{ml}$ Cefotaxime and kept under the same growth conditions.

\section{Nodulation assays}




\section{Chapter 5}

Plants harbouring transgenic roots were inoculated with Bradyrhizobium elkanii WUR3 by dipping the roots in a bacterial solution with an optical density of OD600 $=0.15$. Subsequently, plants were placed in $14 \mathrm{~cm}$ round pots containing sterilized sand-granule $(1: 1 \mathrm{v} / \mathrm{v})$ mixture. Plants were fertilized once per week with EKM medium containing $0.2 \mathrm{mM}$ potassium nitrate and two times with water. Nodules were scored 8 weeks post inoculation.

\section{RNA Isolation and $q R T-P C R$}

$P$. andersonii root RNA was isolated from root samples using CTAB extraction buffer (2\% CTAB, 2.5\% PVP-40, $2 \mathrm{M} \mathrm{NaCl}, 100 \mathrm{mM}$ Tris-HCl pH 8.0, 25 mM EDTA pH 8.0 and 2\% of $\beta$ mercaptoethanol) followed by a phenol-chloroform-isoamyl alcohol (25:24:1, v/v/v) extraction on ice. Next the RNA was isopropanol precipitated at $-20^{\circ} \mathrm{C}$ and washed with $70 \%$ ethanol. RNA was DNAseI treated and further purified on RNeasy Mini Spin columns (Qiagen). cDNA was synthesized from $1 \mu \mathrm{g}$ total RNA using the i-script cDNA synthesis kit (Bio-Rad, Hercules, USA) as described in the manufacturer protocol. Quantitative RT-PCR has been performed using SYBR green based detection (Bio-Rad, Hercules, USA). Experimental setup and execution have been conducted using a MyIQ optical cycler, according to protocol provided by the manufacturer (Biorad, Hercules, USA). Data analysis was performed using BioRad iQ5 software (BioRad). Baselines were set at $100 \mathrm{RFU}$ to calculate $\mathrm{Ct}$ values. Relative expression of PanNSPland PanNSP2 in control and RNAi knockdown roots was normalized using a $P$. andersonii actin gene PanACT1.

\section{Primers used:}

PaACTIN-qF: CCTCATTGGAATGGAAGCAC, PaACTIN-qR: TTCCAGGAAACATGGTGGAC

PanNSP1-qF: GTTCAAAGGCCGAGAGAGC

PanNSP1-qR: CTCGCACCACTTCTCTTTCC

PanNSP2-qF: CAAAGGTGGTGACAGTGGTG

PanNSP2-qR: GAACAGCGCCGAGTAGTAGTG

\section{Endomycorrhization assay}


Transgenic $P$. andersonii plant roots were inoculated with chemical Glomus inocolum. The plants were watered three times a week, once with Hoagland's solution (Hoagland medium $\begin{array}{lllll}\text { according } & \text { to } & \text { et } & \text { al } & 2012\end{array}$ ) and twice with water. To characterize mycorrhyzation efficiencies, $P$. andersonii roots were collected, and checked at the fluorescent macroscope for transgenic roots. Transgenic roots were then submerged in $10 \% \mathrm{KOH}$ and heated at $90^{\circ} \mathrm{C}$ for $20 \mathrm{~min}$. After two times rinsing the roots with water they were heated at $90{ }^{\circ} \mathrm{C}$ for $4 \mathrm{~min}$ in Trypan blue staining solution (2\% Trypan blue in Lactoglycerol) and subsequently transferred to $30 \%$ glycerol. Root fragments were mounted on slides for examination. Mycorrhizal infection was quantified according to Pearson et al method (Tisserant et al., 1993).

\section{Nodule micro sectioning}

Nodules were fixed in phosphate buffer solution (PBS) with $0.25 \%$ glutaraldehyde(Ivanov et al., 2012) included. Vacuum was applied for 1-2 h until tissues sat on the bottom. The tissues were incubated at $4{ }^{\circ} \mathrm{C}$ for overnight. After two times washing with PBS, dehydration steps were performed with $10 \%, 30 \%, 50 \%, 70 \%, 90 \%$ and $100 \%$ ETOH respectively for $10 \mathrm{~min}$ at room temperature for each step. Plastic infiltration was done in four steps, which included solution A (100ml Technovit7100, 1pack HrdnerI, 2.5ml PEG400):100\% ETOH in 1:3, 1:1, 3:1 ratio respectively for $30-60 \mathrm{~min}$ in room temperature and finally treated with $100 \%$ solution A for overnight at $4{ }^{\circ} \mathrm{C}$. All materials were transferred into cupules and nodules located in the middle. Solution A was removed and polymerization solution (15 ml Solution A, $1 \mathrm{ml}$ Hardener II) was added immediately. To remove air from the samples, cupules were covered with parafilm and left for overnight at room temperature. After polymerization, holders were put on the blocks and holding solution (technovit3040: 2 part powder, 1 part liquid) was added from the hole located in the centre of holder and kept for $15 \mathrm{~min}$ at room temperature. Finally sectioning of nodules was performed using a microtome machine and the samples were analysed by microscopy (Leica) after staining with toluidine blue $(0.5 \%)$ buffer and washing with tap water for 5 minutes. 


\section{Chapter 5}

\section{References}

Akiyama, K., et al. (2005). "Plant sesquiterpenes induce hyphal branching in arbuscular mycorrhizal fungi." Nature 435(7043): 824-827.

Ané, J.-M., et al. (2004). "Medicago truncatula DMI1 required for bacterial and fungal symbioses in legumes." Science 303(5662): 1364-1367.

Behm, J. E., et al. (2014). "Parasponia: a novel system for studying mutualism stability." Trends in Plant Science 19(12): 757-763

Besserer, A., et al. (2006). "Strigolactones stimulate arbuscular mycorrhizal fungi by activating mitochondria." PLoS Biol 4(7): e226.

Delaux, P. M., et al. (2013). "NSP1 is a component of the Myc signaling pathway." New Phytologist 199(1): 59-65.

Den Camp, R. O., et al. (2011). "LysM-type mycorrhizal receptor recruited for rhizobium symbiosis in nonlegume Parasponia." Science 331(6019): 909-912.

Doyle, J. J. (2011). "Phylogenetic perspectives on the origins of nodulation." Molecular Plant-Microbe Interactions 24(11): 1289-1295.

Endre, G., et al. (2002). "A receptor kinase gene regulating symbiotic nodule development." Nature 417(6892): $962-$ 966.

Geurts, R., et al. (2016). "What Does It Take to Evolve A Nitrogen-Fixing Endosymbiosis?" Trends in Plant Science 21(3): 199-208.

Gobbato, E. (2015). "Recent developments in arbuscular mycorrhizal signaling." Current Opinion in Plant Biology 26: 1-7.

Gobbato, E., et al. (2012). "A GRAS-type transcription factor with a specific function in mycorrhizal signaling." Current Biology 22(23): 2236-2241.

Heckmann, A. B., et al. (2006). "Lotus japonicus nodulation requires two GRAS domain regulators, one of which is functionally conserved in a non-legume." Plant Physiology 142(4): 1739-1750.

Heckmann, A. B., et al. (2011). "Cytokinin induction of root nodule primordia in Lotus japonicus is regulated by a mechanism operating in the root cortex." Molecular Plant-Microbe Interactions 24(11): 1385-1395.

Hogekamp, C., et al. (2011). "Laser microdissection unravels cell-type-specific transcription in arbuscular mycorrhizal roots, including CAAT-box transcription factor gene expression correlating with fungal contact and spread." Plant Physiology 157(4): 2023-2043.

Horváth, B., et al. (2011). "Medicago truncatula IPD3 is a member of the common symbiotic signaling pathway required for rhizobial and mycorrhizal symbioses." Molecular Plant-Microbe Interactions 24(11): 1345-1358.

Imaizumi-Anraku, H., et al. (2005). "Plastid proteins crucial for symbiotic fungal and bacterial entry into plant roots." Nature 433(7025): 527-531.

Kaló, P., et al. (2005). "Nodulation signaling in legumes requires NSP2, a member of the GRAS family of transcriptional regulators." Science 308(5729): 1786-1789. 


\section{Symbiotic functioning of the GRAS-TYPE transcriptional regulators NSP1 and NSP2 is conserved in the}

Lauressergues, D., et al. (2012). "The microRNA miR171h modulates arbuscular mycorrhizal colonization of Medicago truncatula by targeting NSP2." The Plant Journal 72(3): 512-522.

Lin, K., et al. (2014). "Single nucleus genome sequencing reveals high similarity among nuclei of an endomycorrhizal fungus." PLoS Genet 10(1): e1004078.

Liu, W., et al. (2011). "Strigolactone biosynthesis in Medicago truncatula and rice requires the symbiotic GRAStype transcription factors NSP1 and NSP2." The Plant cell 23(10): 3853-3865.

Maillet, F., et al. (2011). "Fungal lipochitooligosaccharide symbiotic signals in arbuscular mycorrhiza." Nature 469(7328): 58-63.

Marsh, J. F., et al. (2007). "Medicago truncatula NIN is essential for rhizobial-independent nodule organogenesis induced by autoactive calcium/calmodulin-dependent protein kinase." Plant Physiology 144(1): 324-335.

Marvel, D. J., et al. (1987). "Rhizobium symbiotic genes required for nodulation of legume and nonlegume hosts." Proceedings of the National Academy of Sciences 84(5): 1319-1323.

Murakami, Y., et al. (2007). "Positional cloning identifies Lotus japonicus NSP2, a putative transcription factor of the GRAS family, required for NIN and ENOD40 gene expression in nodule initiation." DNA research 13(6): 255265.

Murakami, Y., et al. (2013). "Down-regulation of NSP2 expression in developmentally young regions of Lotus japonicus roots in response to rhizobial inoculation." Plant and Cell Physiology 54(4): 518-527.

Parniske, M. (2008). "Arbuscular mycorrhiza: the mother of plant root endosymbioses." Nature Reviews Microbiology 6(10): 763-775.

Roberts, N. J., et al. (2013). "Rhizobial and mycorrhizal symbioses in Lotus japonicus require lectin nucleotide phosphohydrolase, which acts upstream of calcium signaling." Plant Physiology 161(1): 556-567.

Schauser, L., et al. (1999). "A plant regulator controlling development of symbiotic root nodules." Nature 402(6758): 191-195.

Shtark, O. Y., et al. (2016). "Arbuscular mycorrhiza development in pea (Pisum sativum L.) mutants impaired in five early nodulation genes including putative orthologs of NSP1 and NSP2." Symbiosis 68(1-3): 129-144.

Singh, S., et al. (2014). "CYCLOPS, a DNA-binding transcriptional activator, orchestrates symbiotic root nodule development." Cell Host \& Microbe 15(2): 139-152.

Smit, P., et al. (2007). "Medicago LYK3, an entry receptor in rhizobial nodulation factor signaling." Plant Physiology 145(1): 183-191.

Smit, P., et al. (2005). "NSP1 of the GRAS protein family is essential for rhizobial Nod factor-induced transcription." Science 308(5729): 1789-1791.

Soyano, T., et al. (2013). "Nodule inception directly targets NF-Y subunit genes to regulate essential processes of root nodule development in Lotus japonicus." PLoS Genet 9(3): e1003352.

Stracke, S., et al. (2002). "A plant receptor-like kinase required for both bacterial and fungal symbiosis." Nature 417(6892): 959-962. 


\section{Chapter 5}

Streng, A., et al. (2011). "Evolutionary origin of rhizobium Nod factor signaling." Plant Signaling \& Behavior 6(10): $1510-1514$.

Sun, J., et al. (2015). "Activation of symbiosis signaling by arbuscular mycorrhizal fungi in legumes and rice." The Plant cell 27(3): 823-838.

Takeda, N., et al. (2013). "CERBERUS and NSP1 of Lotus japonicus are common symbiosis genes that modulate arbuscular mycorrhiza development." Plant and Cell Physiology 54(10): 1711-1723.

Tisserant, B., et al. (1993). "In planta histochemical staining of fungal alkaline phosphatase activity for analysis of efficient arbuscular mycorrhizal infections." Mycological Research 97(2): 245-250.

van Zeijl, A., et al. (2015). "Rhizobium lipo-chitooligosaccharide signaling triggers accumulation of cytokinins in Medicago truncatula roots." Molecular Plant 8(8): 1213-1226.

Vernié, T., et al. (2015). "The NIN Transcription Factor Coordinates Diverse Nodulation Programs in Different Tissues of the Medicago truncatula Root." The Plant cell 27(12): 3410-3424.

Wang, C., et al. (2013). "Splice variants of the SIP1 transcripts play a role in nodule organogenesis in Lotus japonicus." Plant Molecular Biology 82(1-2): 97-111.

Yano, K., et al. (2008). "CYCLOPS, a mediator of symbiotic intracellular accommodation." Proceedings of the National Academy of Sciences 105(51): 20540-20545.

Yokota, K., et al. (2010). "Function of GRAS proteins in root nodule symbiosis is retained in homologs of a nonlegume, rice." Plant and Cell Physiology 51(9): 1436-1442.

Yoneyama, K., et al. (2012). "How do nitrogen and phosphorus deficiencies affect strigolactone production and exudation?" Planta 235(6): 1197-1207.

Zhang, X.-C., et al. (2015). "Jasmonate signalling in Arabidopsis involves SGT1b-HSP70-HSP90 chaperone complexes." Nature Plants $\mathbf{1}(5)$. 


\section{Chapter 6}

Dual Effect of Ethylene on Root Nodulation of Parasponia andersonii

Maryam Seifi Kalhor, Rens Holmer, Arjan van Zeijl, Ton Bisseling and René Geurts. 


\section{Chapter 6}

\section{Abstract}

Legumes and Parasponia species engage in a mutualistic symbiosis with nitrogen fixing soil bacteria collectively referred to as rhizobia. The consequence of such engagement is the formation of nodules on the plant root system. Although the interaction is beneficial to the plant, the number of nodules is strictly regulated by different mechanisms. In legumes, one of the components which have been discovered to control nodule number is ethylene. It has been shown that inhibitors of ethylene biosynthesis or ethylene perception enhance rhizobium nodulation. Additionally, genetic studies in Medicago truncatula and Lotus japonicus revealed that interference in the ethylene signaling pathway can lead to an uncontrolled number of root nodules. It remains unclear however, whether similar ethylene inhibitory mechanisms are also functional in Parasponia. Here we showed the negative effect of ethylene on root nodulation of Parasponia andersonii. Ethylene insensitivity was induced through RNAi of PanEIN2, a key regulator of ethylene signaling pathway. The PanEIN2 gene expression was reduced up to $80 \%$ resulting in a 20 -fold increase in nodule number compared to control plants, similar as reported for Lotus japonicus. In addition, a novel phenotype was observed, as nodules on PanEIN2 RNAi roots were defected in intracellular infection. Taken together these data support the hypothesis that similar to legumes, ethylene-mediated inhibition of root nodule formation evolved in Parasponia. The occurrence of different phenotypes in Parasponia and legumes nodules also suggests that in Parasponia, ethylene signaling plays a dual role in root nodule formation.

Keywords: Parasponia, Ethylene insensitivity, PanEIN2, intracellular infection. 


\section{Introduction}

The nitrogen fixing rhizobium symbiosis is a biotrophic interaction in which both partners benefit. The plant supplies the rhizobium bacteria with carbon enabling them to use this energy to fix atmospheric nitrogen into ammonia. This newly fixed ammonia can be exploited by the plant, providing it a selective advantage, especially under nitrogen limiting growth conditions. The nitrogen fixing rhizobium symbiosis is a well-known character of many species of the Fabaceae ( 18.000 species of which the vast majority can establish a nitrogen fixing rhizobium symbiosis), but also occurs in the Parasponia genus of the Cannabaceae. Most probable the symbiosis evolved in parallel in both lineages and emerged at different moments in time. Genetic studies in legumes revealed that bacterial induced symbiotic signaling triggered by secreted lipochitooligosaccharide (LCOs) signals intertwine with the plant hormonal networks to establish a novel organ -the root nodule- to host the bacterial symbionts. Among others, ethylene is found to act as a negative regulator of root nodule formation. Here, we focused whether this character is constrained in Parasponia andersonii.

Although, the nitrogen fixing rhizobium symbiosis in legumes evolved about 60 million years ago, this symbiosis in Parasponia is considered to be significantly younger (Behm et al., 2014). The genus Parasponia represents only 5 tropical tree species that are closely related to species of the genus Trema. The 5 Pasasponia species are exclusively found in the Malay Archipellago, were they grow on the nitrogen poor volcanic mountain sloops. Although the age of the Parasponia genus could not be determined due to the lack of fossil data records, several lines of evidence suggest that it is much younger than the legume lineage. First, Parasponia is phenotypically and molecularly very similar to Trema, suggesting it has emerged from a basal Trema species. Second, its root nodules are more basal when compared to legume root nodules. Consequently, Parasponia-rhizobium symbiosis is less advanced and fix nitrogen less effective than in case of legumes (Op den Camp et al., 2012; Behm et al., 2014).

Comparative studies between legumes and Parasponia showed that genetic constraints at least in part have guided the evolution of the rhizobium symbiosis trait in both lineages. For example like legumes rhizobium, the LCO induced signaling network is essential also in case of Parasponia root nodule formation. However, since the rhizobium symbiosis in Parasponia is considered to be much younger that in legumes, it can be hypothesized that only networks have 


\section{Chapter 6}

been recruited that are essential for nodule formation, whereas genetic adaptations that further enhance the symbiotic engagement may not (yet) have occurred in Parasponia.

Rhizobium secreted LCOs are perceived by specific LysM-type receptor kinases (Untergasser et al., 2008). Upon perception, active cytokinins accumulate in the differentiation zone of the root prior first cell divisions that will give birth to a nodule primordium (van Zeijl et al., 2015). A nodule primordium is associated with formation of a local auxin maximum (Takanashi et al., 2011; Imanishi et al., 2014). Formation of such maximum requires cytokinin signaling (Plet et al., 2011). Among other responses, the cytokinin induced signaling may interfere with auxin transport. Such local inhibition of auxin efflux may result in an formation of a local auxin maximum (Plet et al., 2011).

Abscisic acid, jasmonate and ethylene are negative regulators of rhizobium LCO signaling (Sun et al., 2006; Ding et al., 2008). It has been revealed that ethylene is transiently induced by rhizobia during nodule initiation (Ligero et al., 1986; Caba et al., 1998) and negatively affects the process of nodule development. Ethylene also inhibits bacterial infection and determines the radial positioning of the nodule primoridum in legume roots (Prayitno et al., 2006; Penmetsa et al., 2003; Prayitno et al., 2006a). The inhibitory effect of ethylene on nodulation has been pharmacologically studied by exogenous application of ethylene (Goodlass and Smith, 1979; Lee and LaRue, 1992), or through application of the ethylene precursor 1-aminocyclopropane-1carboxylate (ACC), and/or ACC synthase inhibitor aminoethoxyvinylglycine (AVG) (Yu et al., 1979). In legumes, application of ACC or AVG resulted in different effects on root nodule formation. Whereas ACC inhibits formation of this new organ, AVG results in increased nodulation efficiency in legumes (Lee and LaRue, 1992; Peters and Crist-Estes, 1989; Lohar et al., 2009; Gresshoff et al., 2009; Guinel and LaRue, 1992).

Besides pharmacological studies, also genetics revealed a regulatory role of ethylene signaling in nodule formation. Especially ETHYLENE INSENSITIVE 2 (EIN2) commits an important role in regulating nodule numbers. Studies in the non-legume Arabidopsis thaliana (Arabidopsis) revealed that EIN2 acts as a transcriptional modulator upon proteolytic activation. It interacts with specific transcription factors, thereby increasing their stability (Merchante et al., 2015). Mutating the EIN2 orthologous gene in Medicago (named MtSKL) or knocking it down by RNA interference (RNAi) in Lotus japonicus results in an increase in number of root nodules (Miyata 
et al., 2013). Furthermore, in Medicago Mtein2/Mtskl knockout mutant roots display hypernodulating character in the root that are interspersed with zones that lack nodules.

Here we investigated the role of EIN2 in Parasponia root nodule formation. We show that when the P. andersonii EIN2 (PanEIN2) gene is supressed, nodule formation is enhanced. In line with this we conclude that PanEIN2 has negative effect on nodule formation in P. andersonii. This suggests that the negative effect of ethylene signaling on root nodule formation is a generic function, rather than a genetic adaptation in the legume lineage.

\section{Results}

\section{Increased nodulation efficiency in Parasponia upon interference with the ethylene pathway}

In legumes the inhibitory effect of ethylene on nodulation can be modulated by manipulating the ACC (1-aminocyclopropane-1-carboxylic acid) concentration. For example, applying exogenous ACC results in decreased nodulation efficiencies in Medicago, whereas the reverse effect is achieved by exogenous application of the ACC synthase inhibitor AVG (Aminoethoxyvinylglycine) (Penmetsa and Cook, 1997). To test whether this response also occurs in Parasponia, we grew Bradyrhizobium elkanii (strain WUR3) inoculated Parasponia plantlets in vitro, and supplemented the medium either with $10 \mu \mathrm{m} \mathrm{AVG} \mathrm{or} 50 \mu \mathrm{m} \mathrm{ACC}$. This revealed that addition of AVG results in increased nodulation, whereas plants grown on ACC had reduced nodulation efficiency (Figure 1). These results indicated that nodule formation in Parasponia is also controlled, at least in part, by ethylene.

\section{PanEIN2 is a negative regulator of rhizobium root nodule formation}

Using BLAST tools we identified a Parasponia EIN2 homologous gene (PanEIN2) in the draft Parasponia genome sequence (data not shown). PanEIN2 consist of 7 exons and encodes a

protein of 1,294 amino acids. The N-terminal (450 amino acids) of PanEIN2 contains a NRAMP domain, including a hydrophobic core of 13 transmembrane domains, whereas the C-terminal region contains a CEND transcriptional activator domain and a putative nuclear localization signal (1,262-1,288 amino acids). Phylogenetic reconstruction using the EIN2 gene of Medicago, Lotus, Soybean and Arabidopsis revealed that PanEIN2 represents a putative EIN2 orthologous gene (Figure 2). Analysing available RNAseq data sets for Parasponia revealed that PanEIN2 is 


\section{Chapter 6}

ubiquitously expressed in bellow and above grown tissues (Figure 3), which further supports that PanEIN2 represents a functional gene.

(a)
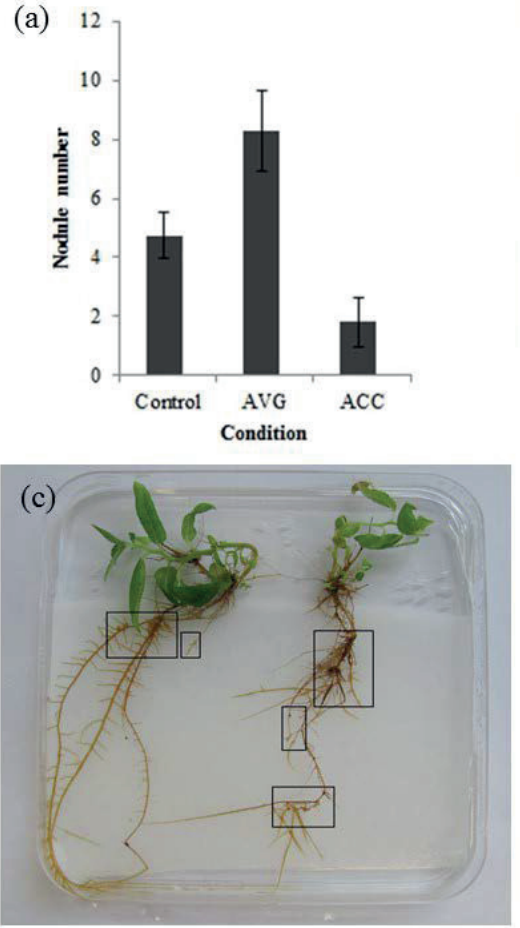

(b)
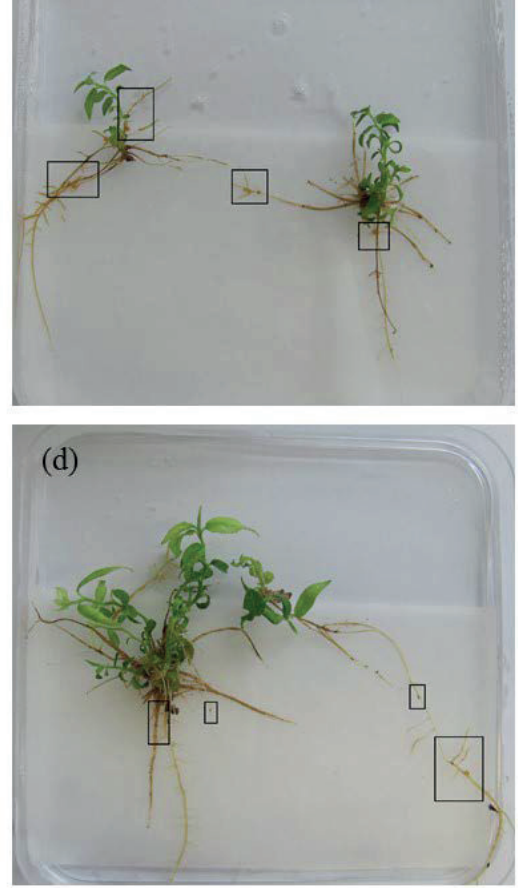

Figure 1. Effects of AVG and ACC on root nodulation of Pararasponia andersonii. Nodule numbers increased by adding AVG and reduced after applying ACC (a). Plants were inoculated with Bradyrhizobium elkanii (WUR3) and grown for 8 weeks at medium supplemented with $0.2 \mathrm{mM}$ Potassium nitrate. Nodules grown on roots incubated with water as Control (b), $10 \mu \mathrm{m} \mathrm{AVG} \mathrm{(c)} \mathrm{and} 50 \mu \mathrm{m}$ ACC (d). Boxes represent the nodules formed on the roots. 


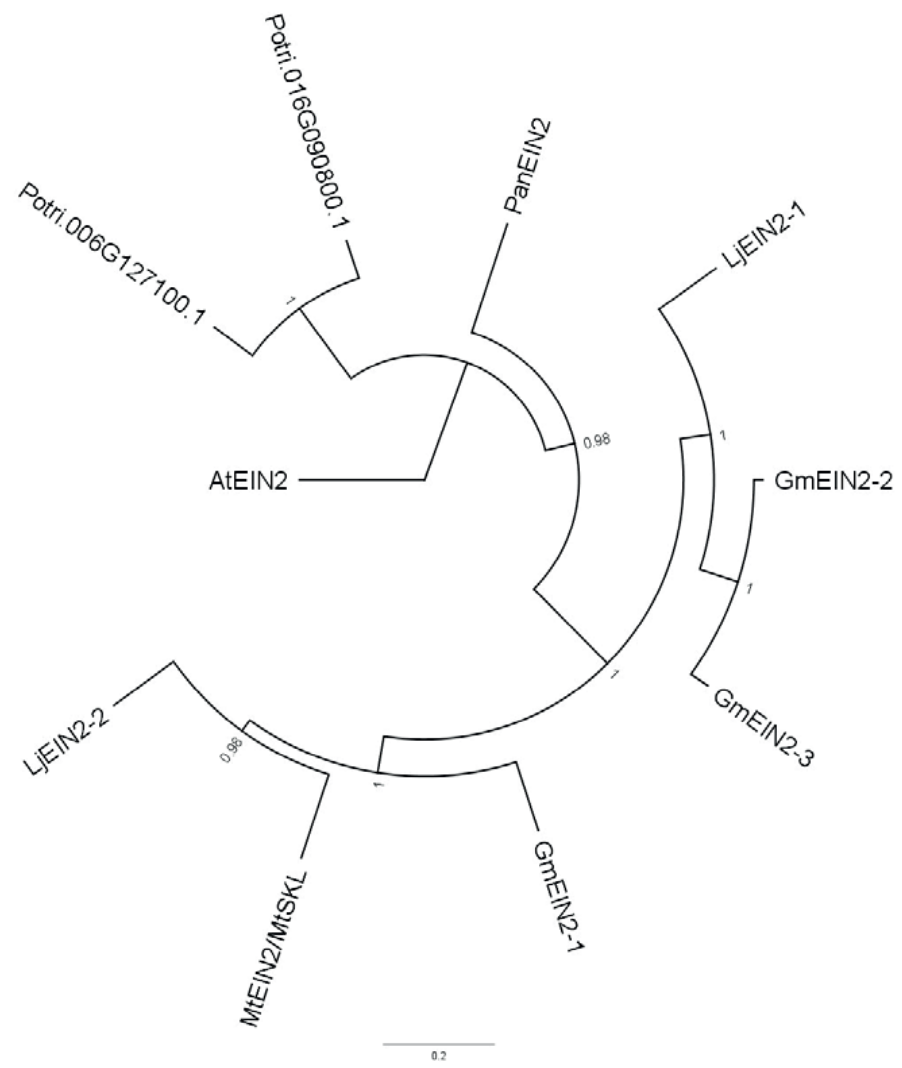

Figure 2. Phylogeny analysis of PanEIN2. Phylogenetic tree showing the relation of PanEIN2 to EIN2 orthologous genes of Medicago, Lotus, soybean and Arabidopsis. 


\section{Chapter 6}

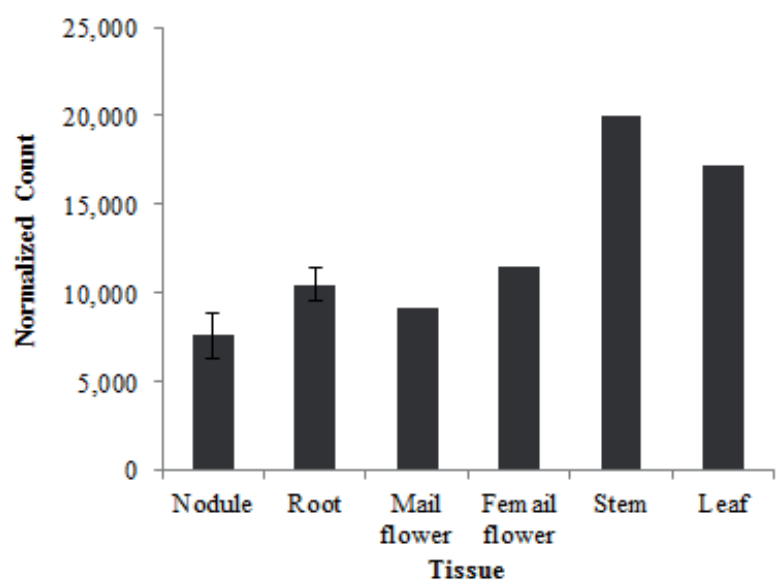

Figure 3. Expression patterns of PanEIN2 in different tissues. Expression of PanEIN2 based on Parasponia RNAseq expression atlas. PanEIN2 expression was detected in nodules, roots, female flowers, male flowers, stem and leaf tissues. Expression counted based on average of 3 biological replicates for nodule and root, and single library for flowers, stem and leaf.

To assess the effect of ethylene on nodulation we performed a RNAi PanEIN2 knockdown study using A. rhizogenes mediated root transformation of Parasponia transgenic roots which showed on average $>50 \%$ reduction in PanEIN2 transcripts (Figure 4). To determine whether reduced expression of PanEIN2 affects nodulation efficiency, $P$. andersonii plantlets containing transgenic PanEIN2 RNAi roots were grown in vitro and inoculated with B. elkanii WUR3. Eight weeks post inoculation we observed a 20 -fold increase in nodule number on PanEIN2 RNAi roots when compared to empty vector control roots (Figure 5a). This shows that the inhibitory role of ethylene signaling in root nodule formation is conserved in Parasponia and legumes.

\section{PanEIN2 knocked down inhibits intracellular infection in Parasponia root nodules}

We noted that nodules formed on PanEIN2 RNAi roots are relatively small. Control plants (transformed with empty vector) harboured big and multi lobed nodules that were present on older parts of the root. In contrast, in the ethylene-insensitive transgenic PanEIN2 RNAi roots hundreds of small, white, single lobed nodules were distributed all over the root (Figure 6). Determining nodule fresh weights revealed that average of PanEIN2 RNAi nodules were only 
$\sim 20 \%$ of the size of nodules in wild type plants (Figure $5 \mathrm{~b}$ ). Therefore, we planned to study ontology of PanEIN2 nodules. To do so, plastic imbedded nodules were sectioned and toluidine blue stained. Nodules formed in control plants were fully colonized, containing many cells harbouring fixation threads (Figure 7e-f). In contrast, nodules formed on PanEIN2 knocked down roots did not contain infected cells. Only intercellular infections were observed (Figure 7ad). This shows that a functional ethylene signaling pathway is essential for intracellular infection of Parasponia nodules.

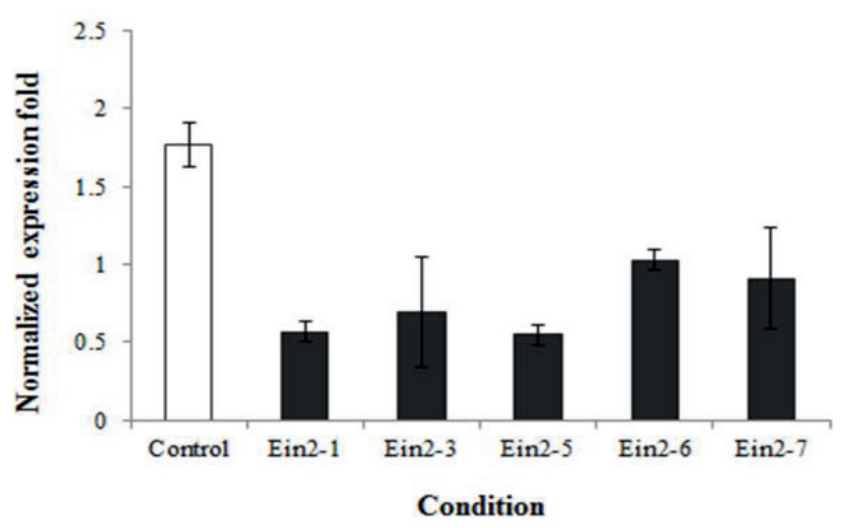

Figure 4. PanEIN2 expression in control (white bar) and PaEIN2 RNAi knockdown roots (black bars, individual EIN2 Knocked down plants). Shown are expression analysis of independently transformed roots containing PanEIN2 RNAi construct. 


\section{Chapter 6}
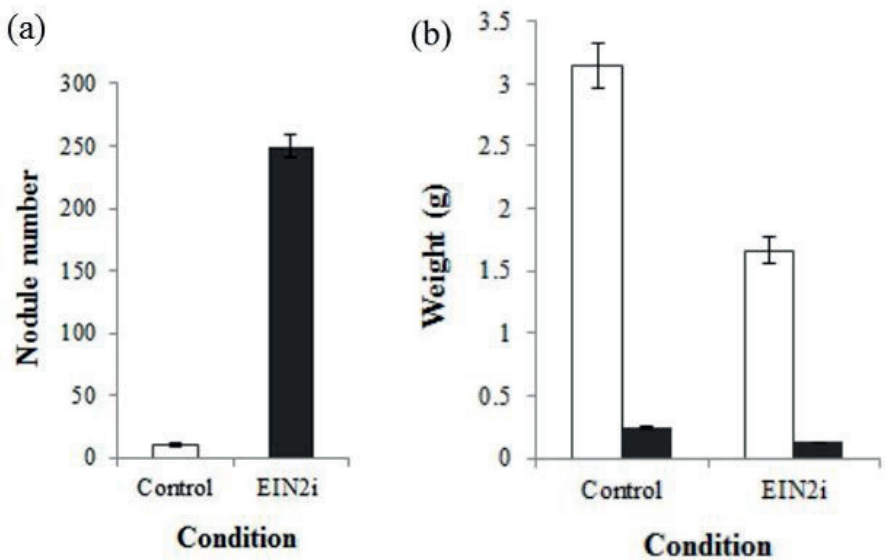

Figure 5. Average number of nodules per transgenic root in control (white bar) and PanEIN2 knocked down (black bar) in Parasponia plants (a). Nodule fresh weight (white bar) and dry weight (black bar) in control (transformed with empty vector) and PanEIN2 knocked down plants (b).
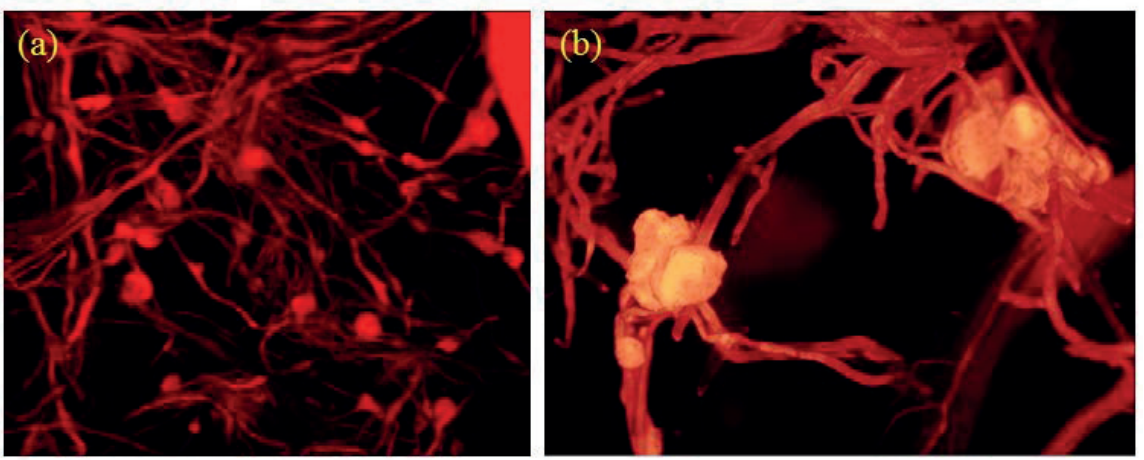

Figure 6. Nodulation phenotype of the Parasponia andersonii PanEIN2 RNAi mutant (a) and Control (b) (transformed with empty vector). Nodules are monitored 8 weeks after inoculation with Bradyrhizobium elkanii (WUR3) and selected based on red florescence due to DsRED1 expression. 


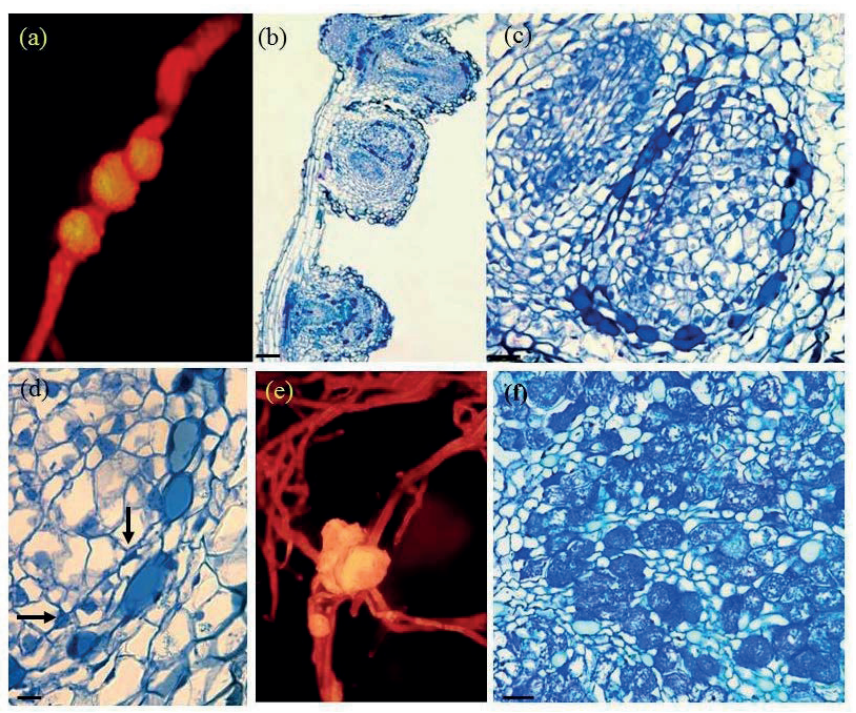

Figure 7. Nodulation and infection phenotype of the Parasponia anadersonii EIN2 mutants. Longitudinal section of control and EIN2 RNAi knock down in Parasponia plants. (a) Nodule formed on transgenic Parasponia PanEIN2 knocked down mutants (selected based on red florescence due to DsRED1 expression). Scale bar: 0.5 mm. (b) Cross section of nodule on mutant plants. Scale bar $75 \mu \mathrm{m}$. (c) Longitudinal section of nodule on PanEIN2 knocked down plants. Scale bars $50 \mu \mathrm{m}$. (d) detail of panel c, only intercellular colonization occurred in PanEIN2 RNAi nodules (arrows) and no intracellular infection was observed .Scale bar: $25 \mu \mathrm{m}$. (e) Nodule formed on transgenic Parasponia transformed with empty vector (selected based on red florescence due to DsRED1 expression). Scale bar: $0.5 \mathrm{~mm}$.

(f) Cross section of nodule infected cells on control plants. Scale bars $50 \mu \mathrm{m}$.

\section{Discussion}

In a diverse range of legume species it is reported that ethylene has a negative role in nodulation initiated by rhizobia (Oldroyd et al., 2001; Nukui et al., 2000; Goodlass and Smith, 1979; Lee and LaRue, 1992; Penmetsa and Cook, 1997). Here we provide evidence that ethylene has similar effect on Parasponia root nodule formation. Nodule number dramatically increased upon knockdown of the ethylene signaling gene PanEIN2. In addition, we found that PanEIN2 RNAi nodules are hampered in intracellular infection, a phenotype unknown in legumes. This indicates that in Parasponia ethylene signaling plays a dual role in root nodule formation. 


\section{Chapter 6}

The gaseous hormone ethylene is synthesized via a methionine dependent pathway, in where methionine is enzymatically converted to ethylene in 3 subsequent steps in which methionine is converted in to ethylene with 1-aminocyclopropane-1- carboxylate (ACC) as intermediate. In this biosynthetic pathway production of ACC by ACC synthase is considered the rate limiting step. Application of the ACC synthase inhibitor (AVG) was resulted in an increase in the number of nodules in legumes, whereas the opposite effect was obtained upon application of exogenous ACC (Peters and Crist-Estes, 1989; Schmidt et al., 1999; Penmetsa and Cook, 1997; Nukui et al., 2000). In accordance, in current study similar results were obtained in Parasponia, which shows that ethylene acts as a generic inhibitor of rhizobium root nodule formation.

Although the response of legume species to ethylene can vary, exogenous ethylene generally affects the frequency of nodule primordia formation (Nukui et al., 2000). The underlying molecular mechanisms remain unknown. Studies in pea suggest that transcriptional regulation of the biosynthetic pathway may function as a regulatory mechanism. In situ hybridization studies indicated that ACC oxidase expression is spatially regulated, and in this way restricting the positioning of nodule primordia. Interfering with this spatial regulation -or alternatively the ethylene signaling pathway- affects not only number of root nodules, but also their positioning in respect to the xylem axis in legume root (Heidstra et al., 1997; Penmetsa and Cook, 1997). It remains to be studied whether a similar positioning also in Parasponia correlates with spatial regulation of ethylene biosynthesis.

Studies in Medicago and Lotus revealed different phenotypes when mutating or knocking down EIN2 genes. In Medicago, zones of hyper-nodulating clusters are formed that are interspaced with non-nodulating root segments (Penmetsa and Cook, 1997). In contrast to Medicago that MtEIN2 is a single gene, two paralogous LjEIN2 genes have been identified in Lotus (Miyata et al., 2013). Knocking down both these genes resulted in significantly more nodules when compared to wild type roots, though hypernodulating root segments interspaced with nonnodualting segments have not been reported (Miyata et al., 2013). Similarly, in Parasponia we did not observe such hypernodualting clusters. This indicates that the hypernodulating clusters are a phenotype unique to Medicago. Alternatively, the studies in Lotus (Miyata et al., 2013) and ours in Parasponia are based on RNAi, which may result in some functional EIN2 protein. This may explain the difference in nodulation phenotype with Medicago, which is an E.M.S. knock out mutant. 
Our results showed that in Parasponia PanEIN2 signaling is essential for intracellular infection of nodule cells. Such role for ethylene signaling has not been found in legumes, suggesting a novel function of this highly conserved pathway in Parasponia. However, it remains open whether Parasponia adapted its ethylene signaling cascade, or more specifically, the functioning of EIN2 to form nitrogen fixing root nodules. Studies in Arabidopsis have shown that the Cterminal part of AtEIN2 can act as a transcriptional modulator, there by stabilizing transcription factors AtEIN3 and AtEIL1 (Tsuda and Somssich, 2015). Binding of ethylene to ER-localized ETR-type receptors modulates their phosphorylation activity, although precise regulation remains elusive (Merchante et al., 2013). Ethylene binding results in inactivation of the negative regulatory kinase CTR1, which is associated with the ETR-type receptors. Deactivation of CTR1 triggers proteolytic cleavage of EIN2, allowing the C-terminal domain to commits its function. Identification of the interacting transcription factor complex in Parasponia (and/or legumes) may provide insight to what extend evolution of the ethylene-EIN2 signaling network is constraint in higher plants.

\section{Materials and Methods}

\section{Construction of Parasponia EIN2RNAi vector}

Degenerate primers were designed based on the sequences of AtEIN2 (Arabidopsis thaliana), PtEIN2 (Populus trichocarpa), VvEIN2 (Vitis vinifera), LjEIN2 (Lotus japonicas) and MtEIN2 (Medicago truncatula) in order to amplify EIN2-like genes from P. andersonii: (forward (GATGGRGYTGATGAGGATCT) and reverse (CCCCTGGCTGGTTTDGMAGC)). Subsequent PCR on root cDNA of $P$. andersonii resulted in $226 \mathrm{bp}$ amplicon. Partial sequence of Parasponia EIN2 gene was cloned into a pENTR-D-Topo plasmid (forward (CACCACAGTGTTtGTATCAGAG), reverse (TTTCCATGGCAGCTGAGAATATT)). Subsequent RNAi construct was made by recombination of the amplified region into the binary vector pK7GWIWG2 (II) driven by the CaMV35S promoter as described in Limpens et al. (2005) (Limpens et al., 2004). pK7GWIWG2(II) contains pAtUBQ10::DsRED1 of pRedRoot as selection marker (Limpens et al., 2004). 


\section{Chapter 6}

\section{Plant transformation and nodulation assay}

Agrobacterium rhizogenes-mediated hairy roots transformation was used to transform Parasponia andersonii as described in (Cao et al., 2012). Transgenic roots were selected based on DsRED1 expression. Transgenic roots were transferred to low nutrient EKM [pH 6.6; 100ml Macro elements 10X (g/liter): $\mathrm{KH}_{2} \mathrm{PO}_{4}: 1.2, \mathrm{~K}_{2} \mathrm{HPO}_{4}: 3.6, \mathrm{MgSO}_{4} .7 \mathrm{H}_{2} \mathrm{PO}_{4}: 2.5, \mathrm{Na}_{2} \mathrm{SO}_{4}: 1.0 ; 1 \mathrm{ml}$ Micro elements (mg/100ml): $\mathrm{MnSO}_{4}: 100, \mathrm{ZnSO}_{4} .7 \mathrm{H}_{2} \mathrm{O}: 25, \mathrm{CuSO}_{4} .5 \mathrm{H}_{2} \mathrm{O}: 25, \mathrm{H}_{3} \mathrm{BO}_{3}: 25 ; 1 \mathrm{ml}$ Fe-citrate (15mM); CaSO4.2H2O: 0.25; NH4NO3: 0.02] medium. Plant transformed with empty vector was investigated as control plants. After two weeks, PaEIN2 RNAi and empty vector control plants were transferred to the pots and inoculated with Bradyrhizobium elkanii WUR3 at a final optical density of 0.15 by directly irrigating after planting in the root. The cultured plants were irrigated three times per week; once with EKM medium (supplemented with $0.2 \mathrm{mM}$ potassium nitrate) and two times with water.

\section{Analysing of RNA seq data}

Primary analysis was done based on $100 \mathrm{bp}$ paired-end read sequencing of the Parasponia transcriptome. Reads were mapped with HiSat2 and mapped to the PanEIN2 gene model and counted. Finally raw reads were normalised with DeSeq2 which resulted in normalized counts.

\section{Quantitative RT-PCR}

RNA was isolated from snap-frozen roots samples using the plant RNA kit (E.Z.N.A, Omega Biotek, Norcross, USA) as described in the manufacturer protocol. cDNA was synthesized from $1 \mu \mathrm{g}$ total RNA using i-script cDNA synthesis kit (Bio-Rad, Hercules, USA) as described in the manufacturer protocol. Quantitative RT-PCR has been performed using SYBR green based detection (Eurogentec, Maastricht, the Netherlands). Experimental setup and execution have been conducted using a MyIQ optical cycler, according to protocol provided by manufacturer (Biorad, Hercules, USA). Data analysis was performed using BioRad iQ5 software (BioRad). Baselines were set at $100 \mathrm{RFU}$ to calculate the Ctvalues, $\mathrm{Ct}$ values of 31 and higher were excluded from the analysis.

Primers used:

PaACT-qF CCTCATTGGAATGGAAGCAC 
PaACT-qR TTCCAGGAAACATGGTGGAC

PaEIN2-qF ATGAATCGGCTAAGTCAAGT

PaEIN2-qR GGCTTTCCATAAGTGAGAGG

\section{Microscopy analysis}

Fixation of nodules was performed in 5\% glutaraldehyde (v/v) and 3\% sucrose (w/v) dissolved in phosphate buffer (pH7.0). Vacuum infiltration of this solution was applied for at least 1 hour. Subsequently an ethanol dehydration series was carried out. Plastic infiltration of samples was done in four steps, included solution A $(100 \mathrm{ml}$ Technovit7100, 1pack HrdnerI, $2.5 \mathrm{ml}$ PEG400):100\% ETOH in 1:3, 1:1, 3:1 ratio respectively for 30-60min in room temperature and finally treated with $100 \%$ solution A for overnight at the $4^{\circ} \mathrm{C}$. All material was transferred into cupules and nodules located in the middle, solution A was removed and polymerization solution (15ml Solution A, $1 \mathrm{ml}$ Hardener II) was added immediately. To remove air from the samples, cupules were covered with parafilm and left for overnight at room temperature. After polymerization, holders were put on the blocks and holding solution (technovit3040: 2part powder, 1 part liquid) was added from the hole located in the centre of holder and kept for 15 min at room temperature.

Finally sectioning of nodules was performed using a microtome machine and the samples were analysed by microscopy (Leica) after staining with toluidine blue $(0.5 \%)$ buffer and washing with tap water for 5 minutes.

\section{Application of the ethylene biosynthesis activator and inhibitor and plant inoculation}

ACC (A-3903; Sigma) and AVG (A-1284; Sigma) were dissolved in sterile distillated water and stored at $4{ }^{\circ} \mathrm{C}$. Chemicals were added to $50 \mathrm{~mL}$ autoclaved EKM medium in a final concentration of 50 and $10 \mu \mathrm{M}$ respectively at 45 to $50^{\circ} \mathrm{C}$, mixed, and then poured in petri dishes. Baradyrhizobium elkanii strain was grown by standard procedures for this genus in YMB medium (pH 6.8 (g/liter: Manitol: 10.0, $\mathrm{K}_{2} \mathrm{HPO}_{4}$ : 0.5, $\mathrm{MgSO}_{4} .7 \mathrm{H}_{2} \mathrm{O}: 0.2, \mathrm{NaCl}$ : 0.1, Yeast Extract: $0.5, \mathrm{pH}: 6.8$ ) for three days at $28^{\circ} \mathrm{C}$ with shaking at $250 \mathrm{rpm}$. The optical density of cell culture was determined with nanodrop and centrifuged in $4000 \mathrm{rpm}$ for 10 minutes. The final pallet was dissolved in sterile water and adjusted at the final optical density of 0.15. Parasponia roots were immerged in this solution for few three seconds and cultured in EKM medium for 8 weeks at $28^{\circ} \mathrm{C}$. 


\section{Chapter 6}

\section{References}

1Behm, J.E., Geurts, R., and Kiers, E.T. (2014) Parasponia: a novel system for studying mutualism stability. Trends in plant science $19,757-763$.

Caba, J., Recalde, L., and Ligero, F. (1998) Nitrate-induced ethylene biosynthesis and the control of nodulation in alfalfa. Plant, Cell \& Environment 21, 87-93.

Cao, Q., Den Camp, R.O., Kalhor, M.S., Bisseling, T., and Geurts, R. (2012) Efficiency of Agrobacterium rhizogenes-mediated root transformation of Parasponia and Trema is temperature dependent. Plant Growth Regulation 68, 459-465.

Ding, Y., Kalo, P., Yendrek, C., Sun, J., Liang, Y., Marsh, J.F., Oldroyd, G.E. (2008) Abscisic acid coordinates nod factor and cytokinin signaling during the regulation of nodulation in Medicago truncatula. The Plant Cell 20, 2681-2695.

Goodlass, G. and Smith, K. (1979) Effects of ethylene on root extension and nodulation of pea (\&lt;i\&gt;Pisum sativum\&lt;/igt; L.) and white clover (\&lt;i\&gt;Trifolium repens\&lt;/i\&gt; L.). Plant and Soil 51, $387-$.

Gresshoff, P.M., Lohar, D., Chan, P.-K., Biswas, B., Jiang, Q., Reid, D., . . Stacey, G. (2009) Genetic analysis of ethylene regulation of legume nodulation. Plant signaling \& behavior 4, 818-823.

Guinel, F.C. and LaRue, T.A. (1992) Ethylene inhibitors partly restore nodulation to pea mutant E 107 (brz). Plant Physiology 99, 515-518.

Heidstra, R., Yang, W.C., Yalcin, Y., Peck, S., Emons, A., and Bisseling, T. (1997) Ethylene provides positional information on cortical cell division but is not involved in Nod factor-induced root hair tip growth in Rhizobium-legume interaction. Development 124, 1781-1787.

Imanishi, L., Perrine-Walker, F.M., Ndour, A., Vayssières, A., Conejero, G., Lucas, M., . . Svistoonoff, S. (2014) Role of auxin during intercellular infection of Discaria trinervis by Frankia. Frontiers in plant science 5.

Lee, K.H. and LaRue, T.A. (1992) Exogenous ethylene inhibits nodulation of Pisum sativum L. cv Sparkle. Plant Physiology 100, 1759-1763.

Ligero, F., Lluch, C., and Olivares, J. (1986) Evolution of Ethylene from Roots of $<\mathrm{i}>$ Medicago sativa $</ \mathrm{i}>$ Plants Inoculated with $<\mathrm{i}>$ Rhizobium meliloti $</ \mathrm{i}>$. Journal of plant physiology 125, 361-365.

Lohar, D., Stiller, J., Kam, J., Stacey, G., and Gresshoff, P.M. (2009) Ethylene insensitivity conferred by a mutated Arabidopsis ethylene receptor gene alters nodulation in transgenic Lotus japonicus. Annals of botany 104, $277-285$.

Merchante, C., Alonso, J.M., and Stepanova, A.N. (2013) Ethylene signaling: simple ligand, complex regulation. Current opinion in plant biology 16, 554-560.

Merchante, C., Brumos, J., Yun, J., Hu, Q., Spencer, K.R., Enríquez, P., Alonso, J.M. (2015) Gene-specific translation regulation mediated by the hormone-signaling molecule EIN2. Cell 163, 684-697.

Miyata, K., Kawaguchi, M., and Nakagawa, T. (2013) Two distinct EIN2 genes cooperatively regulate ethylene signaling in Lotus japonicus. Plant and cell physiology 54, 1469-1477. 


\section{Dual effects of ethylene on root nodulation}

Nukui, N., Ezura, H., Yuhashi, K.-I., Yasuta, T., and Minamisawa, K. (2000) Effects of ethylene precursor and inhibitors for ethylene biosynthesis and perception on nodulation in Lotus japonicus and Macroptilium atropurpureum. Plant and Cell Physiology 41, 893-897.

Oldroyd, G.E., Engstrom, E.M., and Long, S.R. (2001) Ethylene inhibits the Nod factor signal transduction pathway of Medicago truncatula. The Plant Cell 13, 1835-1849.

Op den Camp, R.H., Polone, E., Fedorova, E., Roelofsen, W., Squartini, A., Op den Camp, H.J., . . Geurts, R. (2012) Nonlegume Parasponia andersonii deploys a broad rhizobium host range strategy resulting in largely variable symbiotic effectiveness. Molecular Plant-Microbe Interactions 25, 954-963.

Penmetsa, R.V. and Cook, D.R. (1997) A legume ethylene-insensitive mutant hyperinfected by its rhizobial symbiont. Science 275, 527-530.

Penmetsa, R.V., Frugoli, J.A., Smith, L.S., Long, S.R., and Cook, D.R. (2003) Dual genetic pathways controlling nodule number inMedicago truncatula. Plant Physiology 131, 998-1008.

Peters, N.K. and Crist-Estes, D.K. (1989) Nodule formation is stimulated by the ethylene inhibitor aminoethoxyvinylglycine. Plant Physiology 91, 690-693.

Plet, J., Wasson, A., Ariel, F., Le Signor, C., Baker, D., Mathesius, U., . . Frugier, F. (2011) MtCRE1-dependent cytokinin signaling integrates bacterial and plant cues to coordinate symbiotic nodule organogenesis in Medicago truncatula. The Plant Journal 65, 622-633.

Prayitno, J., Rolfe, B., and Mathesius, U. (2006a) The ethylene-insensitive sickle mutant of Medicago truncatula shows altered auxin transport regulation during nodulation. Plant Physiol 142, 168 - 180.

Prayitno, J., Rolfe, B.G., and Mathesius, U. (2006) The Ethylene-Insensitive sickle Mutant of Medicago truncatula Shows Altered Auxin Transport Regulation during Nodulation. Plant Physiology 142, 168-180.

Schmidt, J.S., Harper, J.E., Hoffman, T.K., and Bent, A.F. (1999) Regulation of soybean nodulation independent of ethylene signaling. Plant physiology 119, 951-960.

Sun, J., Cardoza, V., Mitchell, D.M., Bright, L., Oldroyd, G., and Harris, J.M. (2006) Crosstalk between jasmonic acid, ethylene and Nod factor signaling allows integration of diverse inputs for regulation of nodulation.

Takanashi, K., Sugiyama, A., and Yazaki, K. (2011) Involvement of auxin distribution in root nodule development of Lotus japonicus. Planta 234, 73-81.

Tsuda, K. and Somssich, I.E. (2015) Transcriptional networks in plant immunity. New Phytologist 206, 932-947.

Untergasser, A., Bisseling, T., and Geurts, R. (2008) Making rhizobium-infected root nodules. In Prokaryotic symbionts in plants, pp. 45-69, Springer.

van Zeijl, A., den Camp, R.H.O., Deinum, E.E., Charnikhova, T., Franssen, H., den Camp, H.J.O., . . Geurts, R. (2015) Rhizobium lipo-chitooligosaccharide signaling triggers accumulation of cytokinins in Medicago truncatula roots. Molecular plant 8, 1213-1226.

Yu, Y.-B., Adams, D.O., and Yang, S.F. (1979) 1-Aminocyclopropanecarboxylate synthase, a key enzyme in ethylene biosynthesis. Archives of biochemistry and biophysics 198, 280-286. 


\section{Chapter 7}

General Discussion 


\section{Parasponia-Trema a key system to transfer symbiosis to other non-legumes}

Species of the Parasponia genus are the only known non-legumes capable to establish $\mathrm{N}_{2}$ fixing symbiosis with rhizobium bacteria. As Parasponia and legumes are only remotely related, with a last common ancestor $\sim 100$ million years ago, it seems most probable that both lineages have gained the symbiotic trait by independent evolutionary events. Although the Parasponiarhizobium symbiosis was discovered early twenty century (Bernard, 1916), it took till 1973 before Parasponia plants were introduced in the lab for research (Trinick, 1973). The initial research in that time was to investigate whether rhizobium symbiosis is unique to Parasponia, or also occurs with some closely related sister species of the genus Trema. Also, the rhizobiumsymbiosis of Parasponia was compared with legumes to characterize the commonalties and differences.

Parasponia and Trema species are fast growing trees that form pioneer vegetation in the tropics. Species of both genera are morphologically very similar, resulting in several incorrect identifications suggesting that the rhizobium root nodule symbiosis also occurs in Trema sp. (Trinick, 1973; Coventry et al., 1976; Trinick and Galbraith, 1976). In 1978, extensive examination in New Guinea and Java on Trema orientalis failed to find any nodules. Therefore rhizobium symbiotic relationship within the Cannabaceae (that time still part of the Ulmaceae) was restricted to Parasponia (Akkermans et al., 1978). This genus encompasses five species - $P$. andersonii, P. melastomatifolia, $P$. parviflora, . rigida and P. rugosa- that show a restricted geographical distribution in the Malay Archipelago, where they can be found on slopes of volcanic mountains (Figure 1). In contrast, Trema sp. can be found pan-tropically. Compared to Parasponia, Trema sp. is broadly distributed, indicating that their ecological niche is less restricted. 


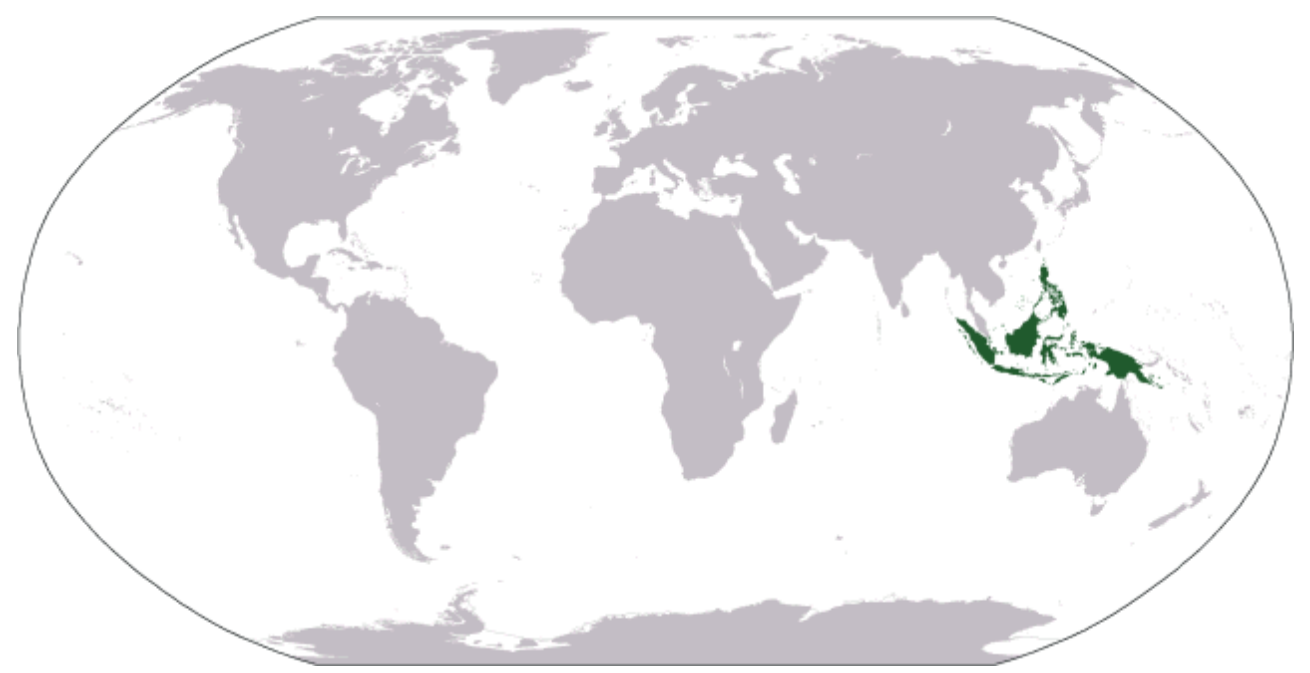

Figure1. Geographical distribution of Parasponia sp..

Recent molecular phylogenetic studies based on four chloroplast genes revealed that the Parasponia lineage is imbedded in the genus Trema (Yang et al., 2013). This further indicates that Parasponia and Trema are closely related. According to current taxonomic rules, Parasponia species may have been included in the genus Trema. Since rhizobium nitrogen fixing symbiosis is exclusively occurring on Parasponia sp., I argue that Parasponia -including it symbiotic trait- evolved relatively recent from an ancestral Trema species. As the legumerhizobium symbiosis is estimated to be $\sim 60$ million years old, I assume that this symbiosis with Parasponia is significantly younger.

Initial studies of field-collected nodules of 3 Parasponia species suggests that these plants are exclusively nodulated by Bradyrhizobium sp. (Trinick, 1980). However, experimental work in the laboratory revealed a much broader host range for $P$. andersonii indicating high promiscuity (Op den Camp et al., 2012). Cross-inoculation experiments of these strains with legumes reveal that Parasponia and legumes can form root nodules with the same rhizobial strains. This indicates that the underlying mechanisms of nodule formation, intracellular infection, nitrogen fixation and establishing a symbiotic interface to exchange nutrients are largely conserved. Therefore I hypothesize that genetic constraints have guided the evolution of the nitrogen fixing rhizobium symbiosis trait in both lineages. A comparison of Parasponia to legumes will provide insights in these constrains. Additionally, as the symbiosis of Parasponia is anticipated to be 
much younger than the symbiosis in legumes, Parasponia-Trema can be used as a comparative system of highly similar plant species that differs in the nitrogen fixing symbiosis trait.

Although the Paraponia and Trema genera only represent tropical trees, they set seeds within 6 to 9 months after planting (Becking, 1992). Nevertheless, as experimental system for laboratory experiments, it is essential that in vitro propagation and transformation protocols are established. For Parasponia such methods have been established already in the 90 decade, and these protocols recently have been extended to Trema tomentosa (Davey et al., 1993; Cao et al., 2012). Additionally, transformation protocols have been established for both species. Initially these protocols focussed on Agrobacterium rhizogenes-based root transformation. This method is commonly used in legumes, as it is relatively fast. Several studies indicated that $A$. rhizogenesmediated root transformation can be used on P. andersonnii and T. tomentosa as well (den Camp et al., 2011; Cao et al., 2012; Granqvist et al., 2015) (Chapter 2, 4, 5 and 6 in this thesis). However, A. rhizogenes mediated transformation systems have several disadvantages; including phenotypic variation between individual roots and the presence of the bacterial rol genes that affect the plant hormone homeostasis. To overcome this problem Agrobacterium tumefaciensbased stable transformation can be applied. I tested whether such method is applicable for $T$. tomenotsa, and found that generating stably transformed plants is relatively straightforward (Chapter 3). In combination with the in vitro propagation protocols, an unlimited number of clones can be generated from primary transformed plant lines. This reduces the importance of generative propagation, and will allow affective research on this tree species. To establish Parasponia-Trema as a comparative research system it will be essential to establish a similar effective transformation protocol for Parasponia.

\section{Distinct effect of nitrate on Parasponia-rhizobium interaction}

Nitrate retards the development of nodules on all legume species (Streeter and Wong, 1988; Barbulova et al., 2007; Sagan et al., 1995). Nitrate is also responsible for decreasing the level of nitrogen fixation in existing nodules (Carroll and Gresshoff, 1983; Fujikake et al., 2002). In our study on the effect of exogenous nitrate on root nodulation in Parasponia (Chapter 4) we initially addressed the question whether similar responses as reported for legumes can be also observed in Parasponia. We found that nodulation efficiencies of Parasponia are reduced in presence of exogenous nitrate, but even at a concentration of $50 \mathrm{mM} \mathrm{KNO}_{3}$ still a few nodules 
were formed. Strikingly, such nodules formed in presence of high nitrate level $\left(>10 \mathrm{mM} \mathrm{KNO}_{3}\right)$ are defected to fix nitrogen, due to impaired intracellular infection. Although these nitrate grown nodules all contained infected cells, the formation of fixation threads was hampered. Transition from infection thread to fixation thread formation is a developmental switch that controls the adaptation of host cells to accommodate rhizobia with whom it aims to exchange nutrients. Fixation threads form a major expansion inside the cell interior, which requires modulation of the plant vacuole. In Parasponia nodule cells that are infected with rhizobium the vacuoles shrink, get fragmented and even can disappear completely, similar as reported for legumes that host rhizobia as transient nitrogen-fixing organelle-like structures (Gavrin et al., 2014). Fixation threads are largely deprived from cell wall structure, allowing a better exchange of nutrients between both partners. Absence of this interface between plant and microbe defects the symbiosis, and may therefore be a very effective way to avoid exploitation by the biotrophic rhizobial microbes.

Why the mechanism of microbial control at the level of the formation of the symbiotic stage has not been found in legumes may have several reasons. First, this mechanism may be functional only in species that form fixation threads. This mode of infection is not exclusive for Parasponia, but also occur in Andira and some Chamaecrista species (de Faria et al., 1986; Naisbitt et al., 1992). However studies on the effect of exogenous nitrate have been limited to legume crops (Lupin, Chickpea, Soybean and Bean) and the models Medicago truncatula and Lotus japonicus. Therefore, extending these studies to more basal legumes will be relevant. Secondly, legumes may have evolved novel adaptations to control the rhizobium symbiosis, which are not present in Parasponia. For example, exogenous nitrate fully blocks formation of root nodules in the studied legumes (Barbulova et al., 2007; Carroll and Gresshoff, 1983), which makes an additional mechanism to control the microbial partner at a later stage superficial. In contrast to legumes, rhizobium LCO induced signaling is not blocked in Parasponia. Studies presented in this thesis show that LCOs trigger for example calcium oscillations in root epidermal cells of Parasponia roots grown in presence of exogenous nitrate. An activated LCO signaling machinery will ultimately lead to the onset of root nodule organogenesis.

The rhizobium symbiosis in legumes as well as Parasponia is founded on the much older arbuscular mycorrhzal symbiosis (Szczyglowski and Amyot, 2003). Genetic studies identified so-called common symbiosis signaling genes that are essential for rhizobium and arbuscular 
mycorrhizal induced signaling (Parniske, 2008). In line with this, is the finding that rhizobium and mycorrhizal fungi produce structurally very similar LCO signaling molecules (Gough and Cullimore, 2011). In model legumes LysM-type receptor kinases have been identified that specially recognize rhizobium LCOs, whereas these receptors are not essential for mycorrhzae. This indicates that perception mechanisms of rhizobium and mycorrhizal signals have been diverged in course of legume evolution. Since the Parasponia-rhizobium symbiosis is considered to be much younger, it can be anticipated that neofunctionalization of rhizobium specific features is less advanced when compared to legumes. This hypothesis is supported by the finding from a LysM-type receptor-PaNFP- that commits a dual function in rhizobium and endomycorrhizal symbiosis (den Camp et al., 2011).

Abuscular mycorrhization and nodulation are very different symbioses in the terms of host responses and microbe involvement (Kosuta et al., 2003). Above all, arbuscular mycorrhizal fungi do not trigger cell divisions in the root cortex. However, in both interactions intracellular hosting of the microsymbiont is key to establish a functional symbiosis (Oldroyd et al., 2009). In case of mycorrhizal fungi this leads to highly branched membrane compartments -known as arbuscules- in root cortical cells. These arbuscules facilitate nutrient exchange. The cellular machinery controlling intracellular hosting of arbuscular mycorrhizal fungi or rhizobium bacteria is very similar. For example, in both symbionts the same vascular and membrane target SNARE proteins are used (Limpens et al., 2009).

Arbuscular mycorrhizal symbiosis is established when the plant experience nutrient deficiencies, especially phosphates. Arbuscular mycorrhzal fungi also facilitate the uptake of exogenous nitrates and supply them to the plant host in return for carbohydrates. However, the underlying mechanisms that plants are used to manipulate this flux of nutrients remain largely unknown. Since the rhizobium nitrogen fixing root nodule symbiosis is founded on arbuscular mycorrhizal symbiosis, it is tempting to speculate that the mechanism employed by Parasponia to control rhizobium at presence of high exogenous nitrates is derived from arbuscular mycorrhiza.

\section{Shared features of the Parasponia and Legume rhizobium symbioses}

To identify shared functional features between legumes and Parasponia in respect to rhizobium symbiosis, it was studied whether the transcriptional regulators NODULATION SIGNALING PATHWAY1 (NSP1) and NSP2 commit symbiotic functions in Parasponia. NSP1 and NSP2 
belong to the GRAS proteins; a conserved family of plant-specific transcriptional regulators that play diverse roles in root and shoot development. In the legumes Medicago and Lotus NSPI and NSP2 are essential for rhizobium LCO signaling and nodule formation (Kaló et al., 2005; Hirsch et al., 2009; Heckmann et al., 2006). Furthermore, knockout mutants of Medicago or Lotus nspl or $n s p 2$ display mild mycorrhizal phenotypes in which the nsp1 and nsp2 mutants show reduced hyphal colonization (Takeda et al., 2013; Maillet et al., 2011). In addition it was found that both proteins are essential transcriptional regulators of $D W A R F 27$, a gene encoding a key enzyme in the strigolactone biosynthesis pathway (Liu et al., 2011). As strigolactones are not only exuded signals that are perceived by arbuscular mycorrhizal fungi, but also endogenous hormones, it may explain why NSP1 and NSP2 are conserved in higher plants, including Arabidopsis (Liu et al., 2011).

In my research I found that $N S P 1$ and $N S P 2$ are also essential for Parasponia root nodule formation, whereas the mycorrhizal infection is only slightly reduced. This phenotype is very similar as reported for Medicago, and underlines that recruitment of both proteins to commit synmbiotic functions are guided by genetic constraints.

The molecular functioning of NSP1 and NSP2 in a symbiotic context remains unclear. Early studies suggested that both proteins are primary response factors that activate transcription upon LCO perception (Smit et al., 2005). This hypothesis was further supported by the identification of NSP1-NSP2 binding sites in the promoter of the MtENOD11 LCO responsive gene. However, other studies indicate that NSP1 and NSP2 are not essential components of the LCO signaling pathway, but rather may function as facilitators (Limpens and Bisseling, 2014). Mutations in such facilitator proteins may affect the readout of the LCO signaling pathway in such a way that symbiotic responses that are associated with root nodule formation are ceased.

\section{Ethylene-mediated negative control of Parasponia nodulation provides insights in genetic constraints underlying rhizobium symbiosis}

In legumes an appropriate level of nitrogen fixing root nodules is beneficial to the host plant, but excessive nodulation diminishes plant growth. Therefore, to control the number of nodules and to maintain proper symbiotic balance, host plants have evolved mechanisms to regulate nodulation. This so-called autoregulation of nodulation (AON) functions in shoots and is based 
on a root-to-shoot long-distance signaling (Nutman, 1952; Pierce and Bauer, 1983; van Brussel et al., 2002). Genetic mutations in AON result in a markedly increased number of nodules.

In addition to the systemic AON mechanism, the gaseous phytohormone ethylene plays important roles in the negative regulation of nodulation. The role of ethylene in physiological processes throughout the life cycle of the plant has been intensively studied. Among other processes, ethylene regulates developmental processes like senescence and abscission, but also responses to biotic stresses that include pathogens and in case of legumes rhizobium.

The inhibitory effect of ethylene on nodulation has been studied pharmacologically as well as genetically. Among the strongest evidence that ethylene signaling affects root nodule formation has been obtained by studying the sickle mutant in Medicago trancatula (Medicago) (Penmetsa and Cook, 1997). SICKLE encodes the Medicago ortholog of EIN2 (ETHYLENE INSENSITIVE2) (Varma Penmetsa et al., 2008). The EIN2 gene was initially identified in $A$. thaliana and the encoded protein plays a central role in signal transmission leading to transcriptional responses (Jun et al., 2004; Alonso et al., 1999; Shibuya et al., 2004; Varma Penmetsa et al., 2008). EIN2 mutants have been generated in several other plant species, including Rice, Petunia, Lotus and Medicago (Jun et al., 2004; Shibuya et al., 2004; Varma Penmetsa et al., 2008). Detailed phenotypic analyses of Medicago sickle/ein2 mutants revealed that ethylene signaling negatively regulates rhizobium LCO-induced oscillations of the nuclear calcium concentration (also known as calcium spiking), LCO induced gene expression, the number of nodules formed, the positioning of nodule primordia in the root, and growth of infection threads (Oldroyd et al., 2001; Penmetsa and Cook, 1997; Heidstra et al., 1997). Similar studies, though less detailed, have been conducted in Lotus. In most plants EIN2 is a single gene that commits the central role in ethylene signaling. However, Lotus harbors 2 paralogous genes of EIN2; LjEIN2-1 and LjEIN2-2, of which the latter is the putative ortholog of MtSKL/MtEIN2. Mutating LjEIN2-2 (also named ENIGMA) affects radial positioning of nodules, but did not result in a hypernodulating phenotype as has been reported for Medicago (Chan et al., 2013). However, by using RNAi to knockdown both paralogous genes nodulation significantly increased (Miyata et al., 2013). This suggests that in Lotus EIN2 functioning in nodulation is sub functionalized over two gene paralogs.

Since the regulatory role of ethylene signaling on root nodulation seems to be conserved in legumes, we investigated the symbiotic role of EIN2 in Parasponia. Like most plant species, and 
in contrast to Lotus, Parasponia contains only a single EIN2 gene. Knocking down of this gene by RNAi resulted in a 20-fold increase of nodules (Chapter 6). This underlines that the negative effect of ethylene on nodulation is not legume specific, but also is present in Parasonia.

The question remains whether the ethylene signaling pathway has been adapted in legumes and Parasponia, and if so, how such adaptations may look like. Till now, knowledge of the molecular functioning of the ethylene signaling pathway has been largely revealed based on studies in Arabidopsis. Ethylene is perceived by a family of ER-localized receptors, which are negative regulators of the signaling pathway (Chang et al., 1993; Hua et al., 1995; Hua and Meyerowitz, 1998; Hua et al., 1998; Sakai et al., 1998). Downstream of these receptors acts the CTR1 kinase that -again as a negative regulator- controls the functioning of EIN2. The membrane localized EIN2 protein is a central transducer of the ethylene signal (Kieber et al., 1993; Alonso et al., 1999). If CTR1 is inactivated and cannot phosphorylate EIN2, this protein is proteolitically activated resulting in release of its $\mathrm{C}$ terminal part. The EIN2 $\mathrm{C}$ terminus is translocated to nucleus where it will activate a transcriptional network (Ju et al., 2012; Qiao et al., 2012; Wen et al., 2012). In the absence of ethylene, the active receptors recruit CTR1 to phosphorylate the C-terminal domain of EIN2 to repress the downstream ethylene response.

To commits its regulatory function in root nodule formation the ethylene pathway could have experience adaptations at several levels. However, only three evolutionary scenarios can explain the functioning of the ethylene signaling in nodulation. (I.) The ethylene signaling pathway has not been adapted in legumes and Parasponia, but its negative role on nodulation is a pleiotropic effect of its generic functioning in plant growth and development. To my opinion this is the most unlikely scenario, since slight modulation of the ethylene homeostasis already affects the nodulation efficiency of the plant. (II.) Alternatively, the ethylene signaling cascade has been recruited to control root nodule formation. In such scenario the recruitment occurred in parallel in the Parasponia and legume lineages and may reflect an adaption that evolved after initial birth of the symbiosis. Recruitment of ethylene as negative regulator for nodulation can be achieved by adaptation of the expression domain of ethylene biosynthesis genes. For example, in pea it has been shown that an ACC oxidase gene is expressed in cortical cells opposite phloem poles, which may be causal for the fact that nodules are generally formed opposite xyleme poles (Heidstra et al., 1997). To find support for the hypothesis that such spatial expression pattern is an evolutionary adaptation a comparative study on the expression profile of ethylene 
biosynthesis and signaling genes in roots of legumes and non-legumes will be relevant. Additionally, reverse genetic studies in where expression domains are disturbed will be essential. (III.) A third evolutionary scenario is the adaptation of ethylene signaling pathway in such a way that it affects the readout of the LCO signaling pathway. The LCO signaling pathway is highly conserved in higher plant species as it is also essential for the more ancient and widespread mycorrhizal symbiosis. A central question that remains in rhizobium symbiosis research is which genetic changes have occurred so that upon the activation of this pathway cortical cell divisions are triggered. As ethylene has a negative effect on LCO signaling already at the level of calcium spiking (Oldroyd et al., 2001), it can be envisioned that adaptations in ethylene homeostasis are essential to allow a symbiotic readout of this pathway in such a way that root nodule formation is triggered. To test this hypothesis it will be essential to modulate the ethylene signaling pathway in a non-nodulating plant species and monitor the effect of this modulation on the LCO signaling pathway.

\section{Future Research}

In this thesis I present transformation protocols for T. tomentosa, a close non-symbiotic relative of Parasponia species. Recent studies revealed that $T$. tomentosa does not display calcium oscillation in response to a complex mixture of LCO molecules extracted from the broad host range of strain Sinorhizobium fredii NGR234 (Granqvist et al., 2015). This strain can nodulate hundreds of legume species as well as Parasponia (den Camp et al., 2011; D'Haeze and Holsters, 2002; Pueppke and Broughton, 1999). In line with this S. fredii NGR234 LCOs trigger calcium spiking in Parasponia root hair cells (Granqvist et al., 2015). The absence of this early symbiotic response (calcium spiking can be detected 10 min post LCO application), makes $T$. tomentosa an excellent species in a comparative approach to Parasponia.

I have conducted first experiments aiming to test the third evolutionary scenario on ethylene signaling as described above. I conducted RNAi of T. tomentosa EIN2 using A. rhizogenes transformation. Of the 20 compound plants that have been generated, two contained nodule-like structures on their roots, 8 weeks post inoculation (Figure 2). Sectioning of these nodule like structures indicate massive cell divisions in pericycle and inner cortical cells, which were deprived from rhizobium intracellular infection. Therefore it can't be ruled out that these 
structures were the result of growth deviations (e.g. derived from lateral root primordia). To rule this out the experiment should be reproduced, and additional symbiotic readouts should be monitored; e.g. symbiotic gene expression and/or calcium spiking. Also it is wise to generate stable lines to commit this research, rather than rely on A. rhizogenes transformation. A protocol for this has been presented in this thesis.

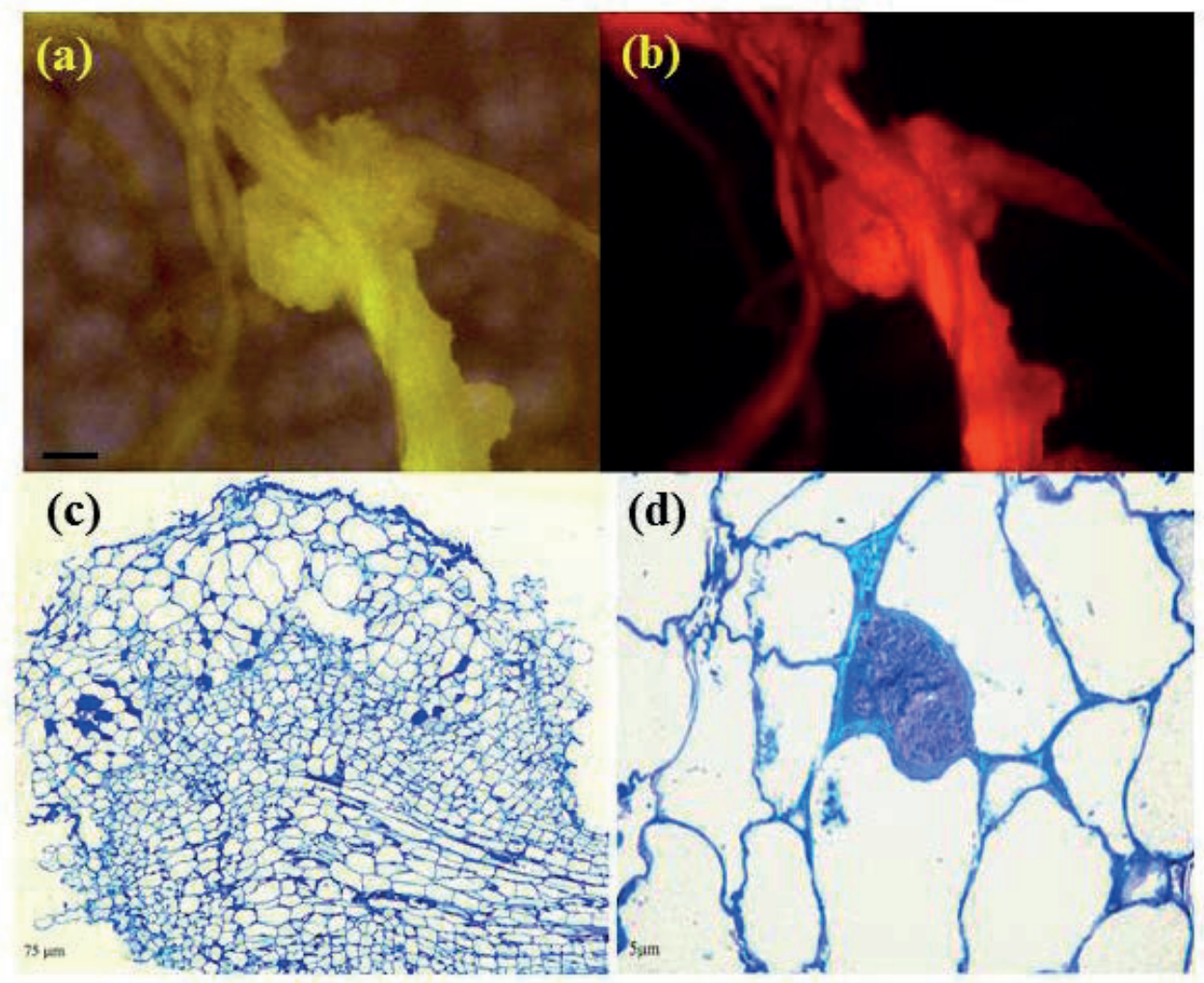

Figure 2. Nodule-like structure on Trema tomentosa TtoEIN2 RNAi knocked down roots. Transgenic roots are selected based on red florescence due to DsRED1 expression. (a-b) Nodule-like structure phenotype on roots. Scale bar: $0.5 \mathrm{~mm}$. (c) Cross section of nodule-like structures. Scale bar $75 \mu \mathrm{m}$. (d) Detail of panel c. Scale bar $5 \mu \mathrm{m}$. Roots monitored 8 weeks after inoculation with Bradyrhizobium elkanii (WUR3). 


\section{Chapter 7}

\section{References}

Akiyama, K., Matsuzaki, K.-i. \& Hayashi, H. (2005). Plant sesquiterpenes induce hyphal branching in arbuscular mycorrhizal fungi. Nature 435(7043): 824-827.

Akkermans, A., Abdulkadir, S. \& Trinick, M. (1978). N2-fixing root nodules in Ulmaceae: Parasponia or (and) Trema spp.? Plant and Soil 49(3): 711-715.

Alonso, J. M., Hirayama, T., Roman, G., Nourizadeh, S. \& Ecker, J. R. (1999). EIN2, a bifunctional transducer of ethylene and stress responses in Arabidopsis. Science 284(5423): 2148-2152.

Ané, J.-M., Kiss, G. B., Riely, B. K., Penmetsa, R. V., Oldroyd, G. E., Ayax, C., Lévy, J., Debellé, F., Baek, J.-M. \& Kalo, P. (2004). Medicago truncatula DMI1 required for bacterial and fungal symbioses in legumes. Science 303(5662): 1364-1367.

Arrighi, J.-F., Barre, A., Amor, B. B., Bersoult, A., Soriano, L. C., Mirabella, R., de Carvalho-Niebel, F., Journet, E.-P., Ghérardi, M. \& Huguet, T. (2006). The Medicago truncatula lysine motif-receptor-like kinase gene family includes NFP and new nodule-expressed genes. Plant Physiology 142(1): 265-279.

Barbulova, A., Rogato, A., D'Apuzzo, E., Omrane, S. \& Chiurazzi, M. (2007). Differential effects of combined N sources on early steps of the Nod factor-dependent transduction pathway in Lotus japonicus. Molecular Plant-Microbe Interactions 20(8): 994-1003.

Beatty, P. H. \& Good, A. G. (2011). Future prospects for cereals that fix nitrogen. Science 333(6041): 416-417.

Becking, J. (1992).The Rhizobium symbiosis of the nonlegume Parasponia. 497-559: Routledge: Chapman and Hall, NY, USA.

Behm, J. E., Geurts, R. \& Kiers, E. T. (2014). Parasponia: a novel system for studying mutualism stability. Trends in Plant Science 19(12): 757-763.

Benková, E., Michniewicz, M., Sauer, M., Teichmann, T., Seifertová, D., Jürgens, G. \& Friml, J. (2003). Local, efflux-dependent auxin gradients as a common module for plant organ formation. Cell 115(5): 591-602.

Bernard, C. (1916). Groene bemesting. Ruijgrok.

Besserer, A., Puech-Pagès, V., Kiefer, P., Gomez-Roldan, V., Jauneau, A., Roy, S., Portais, J.-C., Roux, C., Bécard, G. \& Séjalon-Delmas, N. (2006). Strigolactones stimulate arbuscular mycorrhizal fungi by activating mitochondria. PLoS Biol 4(7): e226.

Boisson-Dernier, A., Chabaud, M., Garcia, F., Bécard, G., Rosenberg, C. \& Barker, D. G. (2001). Agrobacterium rhizogenes-transformed roots of Medicago truncatula for the study of nitrogen-fixing and endomycorrhizal symbiotic associations. Molecular Plant-Microbe Interactions 14(6): 695-700.

Bravo, A., York, T., Pumplin, N., Mueller, L. A. \& Harrison, M. J. (2016). Genes conserved for arbuscular mycorrhizal symbiosis identified through phylogenomics. Nature Plants 2: 15208.

Broghammer, A., Krusell, L., Blaise, M., Sauer, J., Sullivan, J. T., Maolanon, N., Vinther, M., Lorentzen, A., Madsen, E. B. \& Jensen, K. J. (2012). Legume receptors perceive the rhizobial lipochitin oligosaccharide signal molecules by direct binding. Proceedings of the National Academy of Sciences 109(34): 1385913864. 
Buendia, L., Wang, T., Girardin, A. \& Lefebvre, B. (2015). The LysM receptor-like kinase SILYK10 regulates the arbuscular mycorrhizal symbiosis in tomato. New Phytologist.

Burrill, T. J. \& Hansen, R. (1917). Is symbiosis possible between legume bacteria and non-legume plants? Bulletin (University of Illinois (Urbana-Champaign campus). Agricultural Experiment Station); no. 202.

Caba, J., Recalde, L. \& Ligero, F. (1998). Nitrate-induced ethylene biosynthesis and the control of nodulation in alfalfa. Plant, Cell \& Environment 21(1): 87-93.

Cao, Q., Den Camp, R. O., Kalhor, M. S., Bisseling, T. \& Geurts, R. (2012). Efficiency of Agrobacterium rhizogenes-mediated root transformation of Parasponia and Trema is temperature dependent. Plant Growth Regulation 68(3): 459-465.

Carroll, B. J. \& Gresshoff, P. M. (1983). Nitrate inhibition of nodulation and nitrogen fixation in white clover. Zeitschrift für Pflanzenphysiologie 110(1): 77-88.

Carroll, B. J., McNeil, D. L. \& Gresshoff, P. M. (1985). A supernodulation and nitrate-tolerant symbiotic (nts) soybean mutant. Plant Physiology 78(1): 34-40.

Casimiro, I., Marchant, A., Bhalerao, R. P., Beeckman, T., Dhooge, S., Swarup, R., Graham, N., Inzé, D., Sandberg, G. \& Casero, P. J. (2001). Auxin transport promotes Arabidopsis lateral root initiation. The Plant cell 13(4): 843-852.

Cerri, M. R., Frances, L., Kelner, A., Fournier, J., Middleton, P. H., Auriac, M.-C., Mysore, K. S., Wen, J., Erard, M. \& Barker, D. G. (2016). The Symbiosis-Related ERN Transcription Factors Act in Concert to Coordinate Rhizobial Host Root Infection. Plant Physiology: pp. 00230.02016.

Cerri, M. R., Frances, L., Laloum, T., Auriac, M.-C., Niebel, A., Oldroyd, G. E., Barker, D. G., Fournier, J. \& de Carvalho-Niebel, F. (2012). Medicago truncatula ERN transcription factors: regulatory interplay with NSP1/NSP2 GRAS factors and expression dynamics throughout rhizobial infection. Plant Physiology 160(4): 2155-2172.

Chabaud, M., Gherbi, H., Pirolles, E., Vaissayre, V., Fournier, J., Moukouanga, D., Franche, C., Bogusz, D., Tisa, L. S. \& Barker, D. G. (2016). Chitinase-resistant hydrophilic symbiotic factors secreted by Frankia activate both $\mathrm{Ca} 2+$ spiking and NIN gene expression in the actinorhizal plant Casuarina glauca. New Phytologist 209(1): 86-93.

Chan, P. K., Biswas, B. \& Gresshoff, P. M. (2013). Classical Ethylene Insensitive Mutants of the Arabidopsis EIN2 Orthologue Lack the Expected 'hypernodulation'Response in Lotus japonicusF. Journal of Integrative Plant Biology 55(4): 395-408.

Chang, C., Kwok, S. F., Bleecker, A. B. \& Meyerowitz, E. M. (1993). Arabidopsis ethylene-response gene ETR1: similarity of product to two-component regulators. SCIENCE-NEW YORK THEN WASHINGTON-262: 539-539.

Chen, C., Ané, J. M. \& Zhu, H. (2008). OsIPD3, an ortholog of the Medicago truncatula DMI3 interacting protein IPD3, is required for mycorrhizal symbiosis in rice. New Phytologist 180(2): 311-315.

Chen, T., Zhu, H., Ke, D., Cai, K., Wang, C., Gou, H., Hong, Z. \& Zhang, Z. (2012). A MAP kinase kinase interacts with SymRK and regulates nodule organogenesis in Lotus japonicus. The Plant cell 24(2): 823-838. 


\section{Chapter 7}

Chiu, W.-L., Peters, G. A., Levieille, G., Still, P. C., Cousins, S., Osborne, B. \& Elhai, J. (2005). Nitrogen deprivation stimulates symbiotic gland development in Gunnera manicata. Plant Physiology 139(1): 224230 .

Clavijo, F., Diedhiou, I., Vaissayre, V., Brottier, L., Acolatse, J., Moukouanga, D., Crabos, A., Auguy, F., Franche, C. \& Gherbi, H. (2015). The Casuarina NIN gene is transcriptionally activated throughout Frankia root infection as well as in response to bacterial diffusible signals. New Phytologist 208(3): 887-903.

Colpaert, N., Tilleman, S., Van Montagu, M., Gheysen, G. \& Terryn, N. (2008). Composite Phaseolus vulgaris plants with transgenic roots as research tool. African Journal of Biotechnology 7(4).

Cooper, J. B. \& Long, S. R. (1994). Morphogenetic rescue of Rhizobium meliloti nodulation mutants by trans-zeatin secretion. The Plant cell 6(2): 215-225.

Coventry, D., Trinick, M. \& Appleby, C. (1976). A search for a leghaemoglobin-like compound in root nodules ofTrema cannabina lour. Biochimica et Biophysica Acta (BBA)-Protein Structure 420(1): 105-111.

D’Haeze, W. \& Holsters, M. (2002). Nod factor structures, responses, and perception during initiation of nodule development. Glycobiology 12(6): 79R-105R.

Davey, M., Webster, G., Manders, G., Ringrose, F., Power, J. \& Cocking, E. (1993). Effective nodulation of micropropagated shoots of the non-legume Parasponia andersonii by Bradyrhizobium. Journal of Experimental Botany 44(5): 863-867.

Dawson, C. J. \& Hilton, J. (2011). Fertiliser availability in a resource-limited world: Production and recycling of nitrogen and phosphorus. Food Policy 36: S14-S22.

de Faria, S. M., Sutherland, J. M. \& Sprent, J. I. (1986). A new type of infected cell in root nodules of Andira spp.(Leguminosae). Plant Science 45(2): 143-147.

Deinum, E. E., Geurts, R., Bisseling, T. \& Mulder, B. M. (2012). Modeling a cortical auxin maximum for nodulation: different signatures of potential strategies. Front Plant Sci 3: 96.

Delaux, P.-M., Varala, K., Edger, P. P., Coruzzi, G. M., Pires, J. C. \& Ané, J.-M. (2014). Comparative phylogenomics uncovers the impact of symbiotic associations on host genome evolution. PLoS Genet 10(7): e1004487.

Delaux, P. M., Bécard, G. \& Combier, J. P. (2013). NSP1 is a component of the Myc signaling pathway. New Phytologist 199(1): 59-65.

den Camp, R. O., Streng, A., De Mita, S., Cao, Q., Polone, E., Liu, W., Ammiraju, J. S., Kudrna, D., Wing, R. \& Untergasser, A. (2011). LysM-type mycorrhizal receptor recruited for rhizobium symbiosis in nonlegume Parasponia. Science 331(6019): 909-912.

Diédhiou, I., Tromas, A., Cissoko, M., Gray, K., Parizot, B., Crabos, A., Alloisio, N., Fournier, P., Carro, L. \& Svistoonoff, S. (2014). Identification of potential transcriptional regulators of actinorhizal symbioses in Casuarina glauca and Alnus glutinosa. BMC plant biology 14(1): 1.

Dillen, W., Clercq, J., Kapila, J., Zambre, M., Montagu, M. \& Angenon, G. (1997). The effect of temperature on Agrobacterium tumefaciens-mediated gene transfer to plants. The Plant Journal 12(6): 1459-1463. 
Ding, Y., Kalo, P., Yendrek, C., Sun, J., Liang, Y., Marsh, J. F., Harris, J. M. \& Oldroyd, G. E. (2008). Abscisic acid coordinates nod factor and cytokinin signaling during the regulation of nodulation in Medicago truncatula. The Plant cell 20(10): 2681-2695.

Doyle, J. J. (1998). Phylogenetic perspectives on nodulation: evolving views of plants and symbiotic bacteria. Trends in Plant Science 3(12): 473-478.

Doyle, J. J. (2011). Phylogenetic perspectives on the origins of nodulation. Molecular Plant-Microbe Interactions 24(11): 1289-1295.

Drennan, D. \& Norton, C. (1972). The effect of ethrel on nodulation inPisum sativum L. Plant and Soil 36(1-3): 5357.

Elise, S., Etienne-Pascal, J., de Fernanda, C.-N., Gérard, D. \& Julia, F. (2005). The Medicago truncatula SUNN gene encodes a CLV1-like leucine-rich repeat receptor kinase that regulates nodule number and root length. Plant Molecular Biology 58(6): 809-822.

Endre, G., Kereszt, A., Kevei, Z., Mihacea, S., Kaló, P. \& Kiss, G. B. (2002). A receptor kinase gene regulating symbiotic nodule development. Nature 417(6892): 962-966.

Engstrom, E. M. (2011). Phylogenetic analysis of GRAS proteins from moss, lycophyte and vascular plant lineages reveals that GRAS genes arose and underwent substantial diversification in the ancestral lineage common to bryophytes and vascular plants. Plant Signaling \& Behavior 6(6): 850-854.

Estrada-Navarrete, G., Alvarado-Affantranger, X., Olivares, J.-E., Díaz-Camino, C., Santana, O., Murillo, E., Guillén, G., Sánchez-Guevara, N., Acosta, J. \& Quinto, C. (2006). Agrobacterium rhizogenes transformation of the Phaseolus spp.: a tool for functional genomics. Molecular Plant-Microbe Interactions 19(12): 1385-1393.

Faria, S. M. d., McInroy, S. G. \& Sprent, J. I. (1987). The occurrence of infected cells, with persistent infection threads, in legume root nodules. Canadian Journal of Botany 65(3): 553-558.

Ferguson, B. J. \& Mathesius, U. (2003). Signaling interactions during nodule development. Journal of Plant Growth Regulation 22(1): 47-72.

Frugier, F., Kosuta, S., Murray, J. D., Crespi, M. \& Szczyglowski, K. (2008). Cytokinin: secret agent of symbiosis. Trends in Plant Science 13(3): 115-120.

Fujikake, H., Yashima, H., Sato, T., Ohtake, N., Sueyoshi, K. \& Ohyama, T. (2002). Rapid and reversible nitrate inhibition of nodule growth and N2 fixation activity in soybean (Glycine max (L.) Merr.). Soil Science and Plant Nutrition 48(2): 211-217.

Gavrin, A., Kaiser, B. N., Geiger, D., Tyerman, S. D., Wen, Z., Bisseling, T. \& Fedorova, E. E. (2014). Adjustment of host cells for accommodation of symbiotic bacteria: vacuole defunctionalization, HOPS suppression, and TIP1g retargeting in Medicago. The Plant cell 26(9): 3809-3822.

Geurts, R., Fedorova, E. \& Bisseling, T. (2005). Nod factor signaling genes and their function in the early stages of Rhizobium infection. Current Opinion in Plant Biology 8(4): 346-352.

Geurts, R. \& Vleeshouwers, V. G. (2012). Mycorrhizal symbiosis: ancient signalling mechanisms co-opted. Current Biology 22(23): R997-R999. 


\section{Chapter 7}

Geurts, R., Xiao, T. T. \& Reinhold-Hurek, B. (2016). What Does It Take to Evolve A Nitrogen-Fixing Endosymbiosis? Trends in Plant Science 21(3): 199-208.

Gherbi, H., Markmann, K., Svistoonoff, S., Estevan, J., Autran, D., Giczey, G., Auguy, F., Péret, B., Laplaze, L. \& Franche, C. (2008). SymRK defines a common genetic basis for plant root endosymbioses with arbuscular mycorrhiza fungi, rhizobia, and Frankiabacteria. Proceedings of the National Academy of Sciences 105(12): 4928-4932.

Gobbato, E. (2015). Recent developments in arbuscular mycorrhizal signaling. Current Opinion in Plant Biology 26: $1-7$.

Gobbato, E., Marsh, J. F., Vernié, T., Wang, E., Maillet, F., Kim, J., Miller, J. B., Sun, J., Bano, S. A. \& Ratet, P. (2012). A GRAS-type transcription factor with a specific function in mycorrhizal signaling. Current Biology 22(23): 2236-2241.

Gonzalez-Rizzo, S., Crespi, M. \& Frugier, F. (2006). The Medicago truncatula CRE1 cytokinin receptor regulates lateral root development and early symbiotic interaction with Sinorhizobium meliloti. The Plant cell 18(10): 2680-2693.

Goodlass, G. \& Smith, K. (1979). Effects of ethylene on root extension and nodulation of pea (\&lt;i\&gt;Pisum sativum\&lt;/i\&gt; L.) and white clover (\&lt;i\&gt;Trifolium repens\&lt;/i\&gt; L.). Plant and Soil 51(3): $387-$ 395.

Gough, C. \& Cullimore, J. (2011). Lipo-chitooligosaccharide signaling in endosymbiotic plant-microbe interactions. Molecular Plant-Microbe Interactions 24(8): 867-878.

Granqvist, E., Sun, J., Op den Camp, R., Pujic, P., Hill, L., Normand, P., Morris, R. J., Downie, J. A., Geurts, R. \& Oldroyd, G. E. (2015). Bacterial-induced calcium oscillations are common to nitrogen-fixing associations of nodulating legumes and non-legumes. New Phytologist 207(3): 551-558.

Gresshoff, P. M., Lohar, D., Chan, P.-K., Biswas, B., Jiang, Q., Reid, D., Ferguson, B. \& Stacey, G. (2009). Genetic analysis of ethylene regulation of legume nodulation. Plant Signaling \& Behavior 4(9): 818-823.

Grobbelaar, N., Clarke, B. \& Hough, M. (1971). The nodulation and nitrogen fixation of isolated roots of Phaseolus vulgaris L. Plant and Soil 35(1): 203-214.

Groth, M., Takeda, N., Perry, J., Uchida, H., Dräxl, S., Brachmann, A., Sato, S., Tabata, S., Kawaguchi, M. \& Wang, T. L. (2010). NENA, a Lotus japonicus homolog of Sec13, is required for rhizodermal infection by arbuscular mycorrhiza fungi and rhizobia but dispensable for cortical endosymbiotic development. The Plant cell 22(7): 2509-2526.

Guinel, F. C. \& LaRue, T. A. (1992). Ethylene inhibitors partly restore nodulation to pea mutant E 107 (brz). Plant Physiology 99(2): 515-518.

Guo, J., Wang, Y., Song, C., Zhou, J., Qiu, L., Huang, H. \& Wang, Y. (2010). A single origin and moderate bottleneck during domestication of soybean (Glycine max): implications from microsatellites and nucleotide sequences. Annals of Botany 106(3): 505-514.

Gutjahr, C. \& Parniske, M. (2013). Cell and developmental biology of arbuscular mycorrhiza symbiosis. 


\section{General Discussion}

Heckmann, A. B., Lombardo, F., Miwa, H., Perry, J. A., Bunnewell, S., Parniske, M., Wang, T. L. \& Downie, J. A. (2006). Lotus japonicus nodulation requires two GRAS domain regulators, one of which is functionally conserved in a non-legume. Plant Physiology 142(4): 1739-1750.

Heckmann, A. B., Sandal, N., Bek, A. S., Madsen, L. H., Jurkiewicz, A., Nielsen, M. W., Tirichine, L. \& Stougaard, J. (2011). Cytokinin induction of root nodule primordia in Lotus japonicus is regulated by a mechanism operating in the root cortex. Molecular Plant-Microbe Interactions 24(11): 1385-1395.

Heidstra, R., Yang, W. C., Yalcin, Y., Peck, S., Emons, A. \& Bisseling, T. (1997). Ethylene provides positional information on cortical cell division but is not involved in Nod factor-induced root hair tip growth in Rhizobium-legume interaction. Development 124(9): 1781-1787.

Hirakawa, Y., Shinohara, H., Kondo, Y., Inoue, A., Nakanomyo, I., Ogawa, M., Sawa, S., Ohashi-Ito, K., Matsubayashi, Y. \& Fukuda, H. (2008). Non-cell-autonomous control of vascular stem cell fate by a CLE peptide/receptor system. Proceedings of the National Academy of Sciences 105(39): 15208-15213.

Hirel, B., Le Gouis, J., Ney, B. \& Gallais, A. (2007). The challenge of improving nitrogen use efficiency in crop plants: towards a more central role for genetic variability and quantitative genetics within integrated approaches. Journal of Experimental Botany 58(9): 2369-2387.

Hirsch, S., Kim, J., Muñoz, A., Heckmann, A. B., Downie, J. A. \& Oldroyd, G. E. (2009). GRAS proteins form a DNA binding complex to induce gene expression during nodulation signaling in Medicago truncatula. The Plant cell 21(2): 545-557.

Hogekamp, C., Arndt, D., Pereira, P. A., Becker, J. D., Hohnjec, N. \& Küster, H. (2011). Laser microdissection unravels cell-type-specific transcription in arbuscular mycorrhizal roots, including CAAT-box transcription factor gene expression correlating with fungal contact and spread. Plant Physiology 157(4): 2023-2043.

Horváth, B., Yeun, L. H., Domonkos, Á., Halász, G., Gobbato, E., Ayaydin, F., Miró, K., Hirsch, S., Sun, J. \& Tadege, M. (2011). Medicago truncatula IPD3 is a member of the common symbiotic signaling pathway required for rhizobial and mycorrhizal symbioses. Molecular Plant-Microbe Interactions 24(11): 13451358.

Hua, J., Chang, C., Sun, Q. \& Meyerowitz, E. M. (1995). Ethylene insensitivity conferred by Arabidopsis ERS gene. Science 269(5231): 1712 .

Hua, J. \& Meyerowitz, E. M. (1998). Ethylene responses are negatively regulated by a receptor gene family in Arabidopsis thaliana. Cell 94(2): 261-271.

Hua, J., Sakai, H., Nourizadeh, S., Chen, Q. G., Bleecker, A. B., Ecker, J. R. \& Meyerowitz, E. M. (1998). EIN4 and ERS2 are members of the putative ethylene receptor gene family in Arabidopsis. The Plant cell 10(8): 1321-1332.

Huault, E., Laffont, C., Wen, J., Mysore, K. S., Ratet, P., Duc, G. \& Frugier, F. (2014). Local and systemic regulation of plant root system architecture and symbiotic nodulation by a receptor-like kinase. PLoS Genet 10(12): e1004891. 


\section{Chapter 7}

Huo, X., Schnabel, E., Hughes, K. \& Frugoli, J. (2006). RNAi phenotypes and the localization of a protein:: GUS fusion imply a role for Medicago truncatula PIN genes in nodulation. Journal of Plant Growth Regulation 25(2): 156-165.

II, A. (2003). An update of the Angiosperm Phylogeny Group classification for the orders and families of flowering plants: APG II. Botanical Journal of the Linnean Society 141(4): 399-436.

Imaizumi-Anraku, H., Takeda, N., Charpentier, M., Perry, J., Miwa, H., Umehara, Y., Kouchi, H., Murakami, Y., Mulder, L. \& Vickers, K. (2005). Plastid proteins crucial for symbiotic fungal and bacterial entry into plant roots. Nature 433(7025): 527-531.

Imanishi, L., Perrine-Walker, F. M., Ndour, A., Vayssières, A., Conejero, G., Lucas, M., Champion, A., Laplaze, L., Wall, L. \& Svistoonoff, S. (2014). Role of auxin during intercellular infection of Discaria trinervis by Frankia. Frontiers in plant science 5.

Ivanov, S., Fedorova, E. E., Limpens, E., De Mita, S., Genre, A., Bonfante, P. \& Bisseling, T. (2012). Rhizobiumlegume symbiosis shares an exocytotic pathway required for arbuscule formation. Proceedings of the National Academy of Sciences 109(21): 8316-8321.

Ju, C., Yoon, G. M., Shemansky, J. M., Lin, D. Y., Ying, Z. I., Chang, J., Garrett, W. M., Kessenbrock, M., Groth, G. \& Tucker, M. L. (2012). CTR1 phosphorylates the central regulator EIN2 to control ethylene hormone signaling from the ER membrane to the nucleus in Arabidopsis. Proceedings of the National Academy of Sciences 109(47): 19486-19491.

Jun, S.-H., Han, M.-J., Lee, S., Seo, Y. S., Kim, W. T. \& An, G. (2004). OsEIN2 is a positive component in ethylene signaling in rice. Plant and Cell Physiology 45(3): 281-289.

Kahle, J., Baake, M., Doenecke, D. \& Albig, W. (2005). Subunits of the heterotrimeric transcription factor NF-Y are imported into the nucleus by distinct pathways involving importin $\beta$ and importin 13. Molecular and Cellular Biology 25(13): 5339-5354.

Kaló, P., Gleason, C., Edwards, A., Marsh, J., Mitra, R. M., Hirsch, S., Jakab, J., Sims, S., Long, S. R. \& Rogers, J. (2005). Nodulation signaling in legumes requires NSP2, a member of the GRAS family of transcriptional regulators. Science 308(5729): 1786-1789.

Kanamori, N., Madsen, L. H., Radutoiu, S., Frantescu, M., Quistgaard, E. M., Miwa, H., Downie, J. A., James, E. K., Felle, H. H. \& Haaning, L. L. (2006). A nucleoporin is required for induction of Ca2+ spiking in legume nodule development and essential for rhizobial and fungal symbiosis. Proceedings of the National Academy of Sciences of the United States of America 103(2): 359-364.

Karimi, M., Inzé, D. \& Depicker, A. (2002). GATEWAYTM vectors for $<\mathrm{i}>$ Agrobacterium $</ \mathrm{i}>$-mediated plant transformation. Trends in Plant Science 7(5): 193-195.

Kawaguchi, M., Imaizumi-Anraku, H., Koiwa, H., Niwa, S., Ikuta, A., Syono, K. \& Akao, S. (2002). Root, root hair, and symbiotic mutants of the model legume Lotus japonicus. Molecular Plant-Microbe Interactions 15(1): $17-26$

Kerk, N. M., Jiang, K. \& Feldman, L. J. (2000). Auxin metabolism in the root apical meristem. Plant Physiology 122(3): 925-932. 
Kieber, J. J., Rothenberg, M., Roman, G., Feldmann, K. A. \& Ecker, J. R. (1993). CTR1, a negative regulator of the ethylene response pathway in Arabidopsis, encodes a member of the raf family of protein kinases. Cell 72(3): 427-441.

Kistner, C. \& Parniske, M. (2002). Evolution of signal transduction in intracellular symbiosis. Trends in Plant Science 7(11): 511-518.

Kistner, C., Winzer, T., Pitzschke, A., Mulder, L., Sato, S., Kaneko, T., Tabata, S., Sandal, N., Stougaard, J. \& Webb, K. J. (2005). Seven Lotus japonicus genes required for transcriptional reprogramming of the root during fungal and bacterial symbiosis. The Plant cell 17(8): 2217-2229.

Kondo, T., Hasegawa, H. \& Suzuki, M. (2000). Transformation and regeneration of garlic (Allium sativum L.) by Agrobacterium-mediated gene transfer. Plant Cell Reports 19(10): 989-993.

Kosuta, S., Chabaud, M., Lougnon, G., Gough, C., Dénarié, J., Barker, D. G. \& Bécard, G. (2003). A diffusible factor from arbuscular mycorrhizal fungi induces symbiosis-specific MtENOD11 expression in roots ofMedicago truncatula. Plant Physiology 131(3): 952-962.

Larkin, P., Gibson, J., Mathesius, U., Weinman, J., Gartner, E., Hall, E., Tanner, G., Rolfe, B. \& Djordjevic, M. (1996). Transgenic white clover. Studies with the auxin-responsive promoter, GH3, in root gravitropism and lateral root development. Transgenic research 5(5): 325-335.

Lauressergues, D., Delaux, P. M., Formey, D., Lelandais-Brière, C., Fort, S., Cottaz, S., Bécard, G., Niebel, A., Roux, C. \& Combier, J. P. (2012). The microRNA miR171h modulates arbuscular mycorrhizal colonization of Medicago truncatula by targeting NSP2. The Plant Journal 72(3): 512-522.

Lavin, M., Herendeen, P. S. \& Wojciechowski, M. F. (2005). Evolutionary rates analysis of Leguminosae implicates a rapid diversification of lineages during the Tertiary. Systematic biology 54(4): 575-594.

Lee, G.-A., Crawford, G. W., Liu, L. \& Chen, X. (2007). Plants and people from the Early Neolithic to Shang periods in North China. Proceedings of the National Academy of Sciences 104(3): 1087-1092.

Lee, K. H. \& LaRue, T. A. (1992). Exogenous ethylene inhibits nodulation of Pisum sativum L. cv Sparkle. Plant Physiology 100(4): 1759-1763.

Lévy, J., Bres, C., Geurts, R., Chalhoub, B., Kulikova, O., Duc, G., Journet, E.-P., Ané, J.-M., Lauber, E. \& Bisseling, T. (2004). A putative $\mathrm{Ca} 2+$ and calmodulin-dependent protein kinase required for bacterial and fungal symbioses. Science 303(5662): 1361-1364.

Ligero, F., Lluch, C. \& Olivares, J. (1986). Evolution of Ethylene from Roots of $<\mathrm{i}>$ Medicago sativa $</ \mathrm{i}>$ Plants Inoculated with $<\mathrm{i}>$ Rhizobium meliloti $</ \mathrm{i}>$. Journal of plant physiology 125(3): 361-365.

Limpens, E. \& Bisseling, T. (2014). CYCLOPS: a new vision on Rhizobium-induced nodule organogenesis. Cell Host \& Microbe 15(2): 127-129.

Limpens, E., Ivanov, S., van Esse, W., Voets, G., Fedorova, E. \& Bisseling, T. (2009). Medicago N2-fixing symbiosomes acquire the endocytic identity marker Rab7 but delay the acquisition of vacuolar identity. The Plant cell 21(9): 2811-2828. 


\section{Chapter 7}

Limpens, E., Ramos, J., Franken, C., Raz, V., Compaan, B., Franssen, H., Bisseling, T. \& Geurts, R. (2004). RNA interference in Agrobacterium rhizogenes-transformed roots of Arabidopsis and Medicago truncatula. Journal of Experimental Botany 55(399): 983-992.

Lin, K., Limpens, E., Zhang, Z., Ivanov, S., Saunders, D. G., Mu, D., Pang, E., Cao, H., Cha, H. \& Lin, T. (2014). Single nucleus genome sequencing reveals high similarity among nuclei of an endomycorrhizal fungus. PLoS Genet 10(1): e1004078.

Liu, W., Kohlen, W., Lillo, A., den Camp, R. O., Ivanov, S., Hartog, M., Limpens, E., Jamil, M., Smaczniak, C. \& Kaufmann, K. (2011). Strigolactone biosynthesis in Medicago truncatula and rice requires the symbiotic GRAS-type transcription factors NSP1 and NSP2. The Plant Cell Online 23(10): 3853-3865.

Liu, X. \& Widmer, A. (2014). Genome-wide comparative analysis of the GRAS gene family in Populus, Arabidopsis and rice. Plant Molecular Biology Reporter 32(6): 1129-1145.

Lloyd, G. (1980).Commercially-feasible micropropagation of mountain laurel, Kalmia latifolia, by use of shoot-tip culture. In Int. Plant. Prop. Soc., Vol. 30, 421-427.

Lohar, D., Stiller, J., Kam, J., Stacey, G. \& Gresshoff, P. M. (2009). Ethylene insensitivity conferred by a mutated Arabidopsis ethylene receptor gene alters nodulation in transgenic Lotus japonicus. Annals of Botany 104(2): 277-285.

Lohar, D. P., Sharopova, N., Endre, G., Penuela, S., Samac, D., Town, C., Silverstein, K. A. \& VandenBosch, K. A. (2006). Transcript analysis of early nodulation events in Medicago truncatula. Plant Physiology 140(1): 221-234.

Lum, M. R. \& Hirsch, A. M. (2002). Roots and their symbiotic microbes: strategies to obtain nitrogen and phosphorus in a nutrient-limiting environment. Journal of Plant Growth Regulation 21(4): 368-382.

Madsen, E. B., Antolín-Llovera, M., Grossmann, C., Ye, J., Vieweg, S., Broghammer, A., Krusell, L., Radutoiu, S., Jensen, O. N. \& Stougaard, J. (2011). Autophosphorylation is essential for the in vivo function of the Lotus japonicus Nod factor receptor 1 and receptor-mediated signalling in cooperation with Nod factor receptor 5. The Plant Journal 65(3): 404-417.

Madsen, E. B., Madsen, L. H., Radutoiu, S., Olbryt, M., Rakwalska, M., Szczyglowski, K., Sato, S., Kaneko, T., Tabata, S. \& Sandal, N. (2003). A receptor kinase gene of the LysM type is involved in legumeperception of rhizobial signals. Nature 425(6958): 637-640.

Magori, S., Oka-Kira, E., Shibata, S., Umehara, Y., Kouchi, H., Hase, Y., Tanaka, A., Sato, S., Tabata, S. \& Kawaguchi, M. (2009). TOO MUCH LOVE, a root regulator associated with the long-distance control of nodulation in Lotus japonicus. Molecular Plant-Microbe Interactions 22(3): 259-268.

Maillet, F., Poinsot, V., André, O., Puech-Pagès, V., Haouy, A., Gueunier, M., Cromer, L., Giraudet, D., Formey, D. $\&$ Niebel, A. (2011). Fungal lipochitooligosaccharide symbiotic signals in arbuscular mycorrhiza. Nature 469(7328): 58-63.

Malkov, N., Fliegmann, J., Rosenberg, C., Gasciolli, V., Timmers, A. C., Nurisso, A., Cullimore, J. \& Bono, J.-J. (2016). Molecular basis of lipo-chitooligosaccharide recognition by the lysin motif receptor-like kinase LYR3 in legumes. Biochemical Journal 473(10): 1369-1378. 
Marhavý, P., Vanstraelen, M., De Rybel, B., Zhaojun, D., Bennett, M. J., Beeckman, T. \& Benková, E. (2013). Auxin reflux between the endodermis and pericycle promotes lateral root initiation. The EMBO journal 32(1): 149-158.

Markmann, K., Giczey, G. \& Parniske, M. (2008). Functional adaptation of a plant receptor-kinase paved the way for the evolution of intracellular root symbioses with bacteria. PLoS Biol 6(3): e68.

Marsh, J. F., Rakocevic, A., Mitra, R. M., Brocard, L., Sun, J., Eschstruth, A., Long, S. R., Schultze, M., Ratet, P. \& Oldroyd, G. E. (2007). Medicago truncatula NIN is essential for rhizobial-independent nodule organogenesis induced by autoactive calcium/calmodulin-dependent protein kinase. Plant Physiology 144(1): 324-335.

Martín, A. C., Del Pozo, J. C., Iglesias, J., Rubio, V., Solano, R., De La Peña, A., Leyva, A. \& Paz-Ares, J. (2000). Influence of cytokinins on the expression of phosphate starvation responsive genes in Arabidopsis. The Plant Journal 24(5): 559-567.

Martínez-Romero, E. (2009). Coevolution in Rhizobium-legume symbiosis? DNA and cell biology 28(8): $361-370$.

Marvel, D. J., Torrey, J. G. \& Ausubel, F. M. (1987). Rhizobium symbiotic genes required for nodulation of legume and nonlegume hosts. Proceedings of the National Academy of Sciences 84(5): 1319-1323.

Mathesius, U., Schlaman, H. R., Spaink, H. P., Of Sautter, C., Rolfe, B. G. \& Djordjevic, M. A. (1998). Auxin transport inhibition precedes root nodule formation in white clover roots and is regulated by flavonoids and derivatives of chitin oligosaccharides. The Plant Journal 14(1): 23-34.

Mattsson, J., Ckurshumova, W. \& Berleth, T. (2003). Auxin signaling in Arabidopsis leaf vascular development. Plant Physiology 131(3): 1327-1339.

Merchante, C., Alonso, J. M. \& Stepanova, A. N. (2013). Ethylene signaling: simple ligand, complex regulation. Current Opinion in Plant Biology 16(5): 554-560.

Merchante, C., Brumos, J., Yun, J., Hu, Q., Spencer, K. R., Enríquez, P., Binder, B. M., Heber, S., Stepanova, A. N. \& Alonso, J. M. (2015). Gene-specific translation regulation mediated by the hormone-signaling molecule EIN2. Cell 163(3): 684-697.

Messinese, E., Mun, J.-H., Yeun, L. H., Jayaraman, D., Rougé, P., Barre, A., Lougnon, G., Schornack, S., Bono, J.J. \& Cook, D. R. (2007). A novel nuclear protein interacts with the symbiotic DMI3 calcium-and calmodulin-dependent protein kinase of Medicago truncatula. Molecular Plant-Microbe Interactions 20(8): 912-921.

Mitra, R. M., Gleason, C. A., Edwards, A., Hadfield, J., Downie, J. A., Oldroyd, G. E. \& Long, S. R. (2004). A $\mathrm{Ca} 2+/$ calmodulin-dependent protein kinase required for symbiotic nodule development: gene identification by transcript-based cloning. Proceedings of the National Academy of Sciences of the United States of America 101(13): 4701-4705.

Miyata, K., Kawaguchi, M. \& Nakagawa, T. (2013). Two distinct EIN2 genes cooperatively regulate ethylene signaling in Lotus japonicus. Plant and Cell Physiology 54(9): 1469-1477.

Mok, D. W. \& Mok, M. C. (2001). Cytokinin metabolism and action. Annual review of plant biology 52(1): 89-118. 


\section{Chapter 7}

Mortier, V., Den Herder, G., Whitford, R., Van de Velde, W., Rombauts, S., D'haeseleer, K., Holsters, M. \& Goormachtig, S. (2010). CLE peptides control Medicago truncatula nodulation locally and systemically. Plant Physiology 153(1): 222-237.

Murakami, Y., Miwa, H., Imaizumi-Anraku, H., Kouchi, H., Downie, J. A., Kawaguchi, M. \& Kawasaki, S. (2007). Positional cloning identifies Lotus japonicus NSP2, a putative transcription factor of the GRAS family, required for NIN and ENOD40 gene expression in nodule initiation. DNA research 13(6): 255-265.

Murakami, Y., Yokoyama, H., Fukui, R. \& Kawaguchi, M. (2013). Down-regulation of NSP2 expression in developmentally young regions of Lotus japonicus roots in response to rhizobial inoculation. Plant and Cell Physiology 54(4): 518-527.

Murray, J. D., Karas, B. J., Sato, S., Tabata, S., Amyot, L. \& Szczyglowski, K. (2007). A cytokinin perception mutant colonized by Rhizobium in the absence of nodule organogenesis. Science 315(5808): 101-104.

Naisbitt, T., James, E. \& Sprent, J. (1992). The evolutionary significance of the legume genus Chamaecrista, as determined by nodule structure. New Phytologist 122(3): 487-492.

Nap, J.-P. \& Bisseling, T. (1990). Developmental biology of a plant-prokaryote symbiosis: the legume root nodule. Science(Washington) 250(4983): 948-954.

Ng, J. L. P., Hassan, S., Truong, T. T., Hocart, C. H., Laffont, C., Frugier, F. \& Mathesius, U. (2015). Flavonoids and auxin transport inhibitors rescue symbiotic nodulation in the Medicago truncatula cytokinin perception mutant cre1. The Plant cell 27(8): 2210-2226.

Nukui, N., Ezura, H., Yuhashi, K.-I., Yasuta, T. \& Minamisawa, K. (2000). Effects of ethylene precursor and inhibitors for ethylene biosynthesis and perception on nodulation in Lotus japonicus and Macroptilium atropurpureum. Plant and Cell Physiology 41(7): 893-897.

Nutman, P. (1952). Studies on the physiology of nodule formation III. Experiments on the excision of root-tips and nodules. Annals of Botany 16(1): 79-103.

Okamoto, S. \& Kawaguchi, M. (2015). Shoot HAR1 mediates nitrate inhibition of nodulation in Lotus japonicus. Plant Signaling \& Behavior 10(5): e1000138.

Okamoto, S., Ohnishi, E., Sato, S., Takahashi, H., Nakazono, M., Tabata, S. \& Kawaguchi, M. (2009). Nod factor/nitrate-induced CLE genes that drive HAR1-mediated systemic regulation of nodulation. Plant and Cell Physiology 50(1): 67-77.

Okamoto, S., Shinohara, H., Mori, T., Matsubayashi, Y. \& Kawaguchi, M. (2013). Root-derived CLE glycopeptides control nodulation by direct binding to HAR1 receptor kinase. Nature communications 4 .

Oldroyd, G. E., Engstrom, E. M. \& Long, S. R. (2001). Ethylene inhibits the Nod factor signal transduction pathway of Medicago truncatula. The Plant Cell Online 13(8): 1835-1849.

Oldroyd, G. E., Harrison, M. J. \& Paszkowski, U. (2009). Reprogramming plant cells for endosymbiosis. Science 324(5928): 753-754.

Olsson, J. E., Nakao, P., Bohlool, B. B. \& Gresshoff, P. M. (1989). Lack of systemic suppression of nodulation in split root systems of supernodulating soybean (Glycine max [L.] Merr.) mutants. Plant Physiology 90(4): 1347-1352. 


\section{General Discussion}

Op den Camp, R. H., Polone, E., Fedorova, E., Roelofsen, W., Squartini, A., Op den Camp, H. J., Bisseling, T. \& Geurts, R. (2012). Nonlegume Parasponia andersonii deploys a broad rhizobium host range strategy resulting in largely variable symbiotic effectiveness. Molecular Plant-Microbe Interactions 25(7): 954-963.

Ovchinnikova, E., Journet, E.-P., Chabaud, M., Cosson, V., Ratet, P., Duc, G., Fedorova, E., Liu, W., den Camp, R. O. \& Zhukov, V. (2011). IPD3 controls the formation of nitrogen-fixing symbiosomes in pea and Medicago Spp. Molecular Plant-Microbe Interactions 24(11): 1333-1344.

Pacios-Bras, C., Schlaman, H. R., Boot, K., Admiraal, P., Langerak, J. M., Stougaard, J. \& Spaink, H. P. (2003). Auxin distribution in Lotus japonicus during root nodule development. Plant Molecular Biology 52(6): 1169-1180.

Parniske, M. (2008). Arbuscular mycorrhiza: the mother of plant root endosymbioses. Nature Reviews Microbiology 6(10): 763-775.

Penmetsa, R. V. \& Cook, D. R. (1997). A legume ethylene-insensitive mutant hyperinfected by its rhizobial symbiont. Science 275(5299): 527-530.

Penmetsa, R. V., Frugoli, J. A., Smith, L. S., Long, S. R. \& Cook, D. R. (2003). Dual genetic pathways controlling nodule number inMedicago truncatula. Plant Physiology 131(3): 998-1008.

Perrine-Walker, F., Doumas, P., Lucas, M., Vaissayre, V., Beauchemin, N. J., Band, L. R., Chopard, J., Crabos, A., Conejero, G. \& Péret, B. (2010). Auxin carriers localization drives auxin accumulation in plant cells infected by Frankia in Casuarina glauca actinorhizal nodules. Plant Physiology 154(3): 1372-1380.

Peters, N. K. \& Crist-Estes, D. K. (1989). Nodule formation is stimulated by the ethylene inhibitor aminoethoxyvinylglycine. Plant Physiology 91(2): 690-693.

Pierce, M. \& Bauer, W. D. (1983). A rapid regulatory response governing nodulation in soybean. Plant Physiology 73(2): 286-290.

Pietraszewska-Bogiel, A., Lefebvre, B., Koini, M. A., Klaus-Heisen, D., Takken, F. L., Geurts, R., Cullimore, J. V. \& Gadella, T. W. (2013). Interaction of Medicago truncatula lysin motif receptor-like kinases, NFP and LYK3, produced in Nicotiana benthamiana induces defence-like responses. PLoS ONE 8(6): e65055.

Plet, J., Wasson, A., Ariel, F., Le Signor, C., Baker, D., Mathesius, U., Crespi, M. \& Frugier, F. (2011). MtCRE1dependent cytokinin signaling integrates bacterial and plant cues to coordinate symbiotic nodule organogenesis in Medicago truncatula. The Plant Journal 65(4): 622-633.

Prayitno, J., Rolfe, B. \& Mathesius, U. (2006a). The ethylene-insensitive sickle mutant of Medicago truncatula shows altered auxin transport regulation during nodulation. Plant Physiol 142: 168 - 180.

Prayitno, J., Rolfe, B. G. \& Mathesius, U. (2006). The Ethylene-Insensitive sickle Mutant of Medicago truncatula Shows Altered Auxin Transport Regulation during Nodulation. Plant Physiology 142(1): 168-180.

Pueppke, S. G. \& Broughton, W. J. (1999). Rhizobium sp. strain NGR234 and R. fredii USDA257 share exceptionally broad, nested host ranges. Molecular Plant-Microbe Interactions 12(4): 293-318.

Qiao, H., Shen, Z., Huang, S.-s. C., Schmitz, R. J., Urich, M. A., Briggs, S. P. \& Ecker, J. R. (2012). Processing and subcellular trafficking of ER-tethered EIN2 control response to ethylene gas. Science 338(6105): 390-393. 


\section{Chapter 7}

Read, D., Duckett, J., Francis, R., Ligrone, R. \& Russell, A. (2000). Symbiotic fungal associations in 'lower'land plants. Philosophical Transactions of the Royal Society of London B: Biological Sciences 355(1398): 815831.

Reid, D. E., Ferguson, B. J. \& Gresshoff, P. M. (2011). Inoculation-and nitrate-induced CLE peptides of soybean control NARK-dependent nodule formation. Molecular Plant-Microbe Interactions 24(5): 606-618.

Roberts, N. J., Morieri, G., Kalsi, G., Rose, A., Stiller, J., Edwards, A., Xie, F., Gresshoff, P. M., Oldroyd, G. E. \& Downie, J. A. (2013). Rhizobial and mycorrhizal symbioses in Lotus japonicus require lectin nucleotide phosphohydrolase, which acts upstream of calcium signaling. Plant Physiology 161(1): 556-567.

Roth, L. \& Stacey, G. (1989). Bacterium release into host cells of nitrogen-fixing soybean nodules: the symbiosome membrane comes from three sources. European journal of cell biology 49(1): 13-23.

Sagan, M., Morandi, D., Tarenghi, E. \& Duc, G. (1995). Selection of nodulation and mycorrhizal mutants in the model plant Medicago truncatula (Gaertn.) after $\gamma$-ray mutagenesis. Plant Science 111(1): 63-71.

Saito, K., Yoshikawa, M., Yano, K., Miwa, H., Uchida, H., Asamizu, E., Sato, S., Tabata, S., Imaizumi-Anraku, H. \& Umehara, Y. (2007). NUCLEOPORIN85 is required for calcium spiking, fungal and bacterial symbioses, and seed production in Lotus japonicus. The Plant cell 19(2): 610-624.

Sakai, H., Hua, J., Chen, Q. G., Chang, C., Medrano, L. J., Bleecker, A. B. \& Meyerowitz, E. M. (1998). ETR2 is an ETR1-like gene involved in ethylene signaling in Arabidopsis. Proceedings of the National Academy of Sciences 95(10): 5812-5817.

Salas, M., Park, S., Srivatanakul, M. \& Smith, R. (2001). Temperature influence on stable T-DNA integration in plant cells. Plant Cell Reports 20(8): 701-705.

Schauser, L., Roussis, A., Stiller, J. \& Stougaard, J. (1999). A plant regulator controlling development of symbiotic root nodules. Nature 402(6758): 191-195.

Schmidt, J. S., Harper, J. E., Hoffman, T. K. \& Bent, A. F. (1999). Regulation of soybean nodulation independent of ethylene signaling. Plant Physiology 119(3): 951-960.

Schnabel, E., Kassaw, T., Smith, L., Marsh, J., Oldroyd, G. E., Long, S. R. \& Frugoli, J. (2011). ROOT DETERMINED NODULATION 1 regulates nodule number in M. truncatula and defines a highly conserved, uncharacterized plant gene family. Plant Physiology: pp. 111.178756.

Shibuya, K., Barry, K. G., Ciardi, J. A., Loucas, H. M., Underwood, B. A., Nourizadeh, S., Ecker, J. R., Klee, H. J. \& Clark, D. G. (2004). The central role of PhEIN2 in ethylene responses throughout plant development in petunia. Plant Physiology 136(2): 2900-2912.

Shtark, O. Y., Sulima, A. S., Zhernakov, A. I., Kliukova, M. S., Fedorina, J. V., Pinaev, A. G., Kryukov, A. A., Akhtemova, G. A., Tikhonovich, I. A. \& Zhukov, V. A. (2016). Arbuscular mycorrhiza development in pea (Pisum sativum L.) mutants impaired in five early nodulation genes including putative orthologs of NSP1 and NSP2. Symbiosis 68(1-3): 129-144.

Singh, S., Katzer, K., Lambert, J., Cerri, M. \& Parniske, M. (2014). CYCLOPS, a DNA-binding transcriptional activator, orchestrates symbiotic root nodule development. Cell Host \& Microbe 15(2): 139-152. 
Smit, P., Limpens, E., Geurts, R., Fedorova, E., Dolgikh, E., Gough, C. \& Bisseling, T. (2007). Medicago LYK3, an entry receptor in rhizobial nodulation factor signaling. Plant Physiology 145(1): 183-191.

Smit, P., Raedts, J., Portyanko, V., Debellé, F., Gough, C., Bisseling, T. \& Geurts, R. (2005). NSP1 of the GRAS protein family is essential for rhizobial Nod factor-induced transcription. Science 308(5729): 1789-1791.

Soyano, T., Hirakawa, H., Sato, S., Hayashi, M. \& Kawaguchi, M. (2014). NODULE INCEPTION creates a longdistance negative feedback loop involved in homeostatic regulation of nodule organ production. Proceedings of the National Academy of Sciences 111(40): 14607-14612.

Soyano, T., Kouchi, H., Hirota, A. \& Hayashi, M. (2013). Nodule inception directly targets NF-Y subunit genes to regulate essential processes of root nodule development in Lotus japonicus. PLoS Genet 9(3): e1003352.

Sprent, J. I. (2008). 60Ma of legume nodulation. What's new? What's changing? Journal of Experimental Botany 59(5): 1081-1084.

Steen, A., Buist, G., Horsburgh, G. J., Venema, G., Kuipers, O. P., Foster, S. J. \& Kok, J. (2005). AcmA of Lactococcus lactis is an $\mathrm{N}$-acetylglucosaminidase with an optimal number of LysM domains for proper functioning. FEBS Journal 272(11): 2854-2868.

Stracke, S., Kistner, C., Yoshida, S., Mulder, L., Sato, S., Kaneko, T., Tabata, S., Sandal, N., Stougaard, J. \& Szczyglowski, K. (2002). A plant receptor-like kinase required for both bacterial and fungal symbiosis. Nature 417(6892): 959-962.

Streeter, J. \& Wong, P. P. (1988). Inhibition of legume nodule formation and N2 fixation by nitrate. Critical Reviews in Plant Sciences 7(1): 1-23.

Streng, A., op den Camp, R., Bisseling, T. \& Geurts, R. (2011). Evolutionary origin of rhizobium Nod factor signaling. Plant Signaling \& Behavior 6(10): 1510-1514.

Sun, J., Cardoza, V., Mitchell, D. M., Bright, L., Oldroyd, G. \& Harris, J. M. (2006). Crosstalk between jasmonic acid, ethylene and Nod factor signaling allows integration of diverse inputs for regulation of nodulation. The Plant Journal 46(6): 961-970.

Sun, J., Miller, J. B., Granqvist, E., Wiley-Kalil, A., Gobbato, E., Maillet, F., Cottaz, S., Samain, E., Venkateshwaran, M. \& Fort, S. (2015). Activation of symbiosis signaling by arbuscular mycorrhizal fungi in legumes and rice. The Plant cell 27(3): 823-838.

Suzaki, T., Yano, K., Ito, M., Umehara, Y., Suganuma, N. \& Kawaguchi, M. (2012). Positive and negative regulation of cortical cell division during root nodule development in Lotus japonicus is accompanied by auxin response. Development 139(21): 3997-4006.

Sytsma, K. J., Morawetz, J., Pires, J. C., Nepokroeff, M., Conti, E., Zjhra, M., Hall, J. C. \& Chase, M. W. (2002). Urticalean rosids: circumscription, rosid ancestry, and phylogenetics based on rbcL, trnL-F, and ndhF sequences. American Journal of Botany 89(9): 1531-1546.

Szczyglowski, K. \& Amyot, L. (2003). Symbiosis, inventiveness by recruitment? Plant Physiology 131(3): $935-940$.

Takanashi, K., Sugiyama, A. \& Yazaki, K. (2011). Involvement of auxin distribution in root nodule development of Lotus japonicus. Planta 234(1): 73-81. 


\section{Chapter 7}

Takeda, N., Tsuzuki, S., Suzaki, T., Parniske, M. \& Kawaguchi, M. (2013). CERBERUS and NSP1 of Lotus japonicus are common symbiosis genes that modulate arbuscular mycorrhiza development. Plant and Cell Physiology 54(10): 1711-1723.

Tirichine, L., Imaizumi-Anraku, H., Yoshida, S., Murakami, Y., Madsen, L. H., Miwa, H., Nakagawa, T., Sandal, N., Albrektsen, A. S. \& Kawaguchi, M. (2006). Deregulation of a Ca2\&plus;/calmodulin-dependent kinase leads to spontaneous nodule development. Nature 441(7097): 1153-1156.

Tirichine, L., Sandal, N., Madsen, L. H., Radutoiu, S., Albrektsen, A. S., Sato, S., Asamizu, E., Tabata, S. \& Stougaard, J. (2007). A gain-of-function mutation in a cytokinin receptor triggers spontaneous root nodule organogenesis. Science 315(5808): 104-107.

Tisserant, B., Gianinazzi-Pearson, V., Gianinazzi, S. \& Gollotte, A. (1993). In planta histochemical staining of fungal alkaline phosphatase activity for analysis of efficient arbuscular mycorrhizal infections. Mycological Research 97(2): 245-250.

Trinick, M. (1973). Symbiosis between Rhizobium and the non-legume, Trema aspera. Nature 244: 459-460.

Trinick, M. (1979). Structure of nitrogen-fixing nodules formed by Rhizobium on roots of Parasponia andersonii Planch. Canadian Journal of Microbiology 25(5): 565-578.

Trinick, M. (1980). Growth of Parasponia in agar tube culture and symbiotic effectiveness of isolates from Parasponia spp. New Phytologist 85(1): 37-45.

Trinick, M. \& Galbraith, J. (1976). Structure of root nodules formed by Rhizobium on the non-legume Trema cannabina var. scabra. Archives of Microbiology 108(2): 159-166.

Tsuda, K. \& Somssich, I. E. (2015). Transcriptional networks in plant immunity. New Phytologist 206(3): $932-947$.

Ulmasov, T., Murfett, J., Hagen, G. \& Guilfoyle, T. J. (1997). Aux/IAA proteins repress expression of reporter genes containing natural and highly active synthetic auxin response elements. The Plant Cell Online 9(11): 1963-1971.

Untergasser, A., Bisseling, T. \& Geurts, R. (2008).Making rhizobium-infected root nodules. In Prokaryotic symbionts in plants, 45-69: Springer.

van Brussel, A. A., Tak, T., Boot, K. J. \& Kijne, J. W. (2002). Autoregulation of root nodule formation: signals of both symbiotic partners studied in a split-root system of Vicia sativa subsp. nigra. Molecular PlantMicrobe Interactions 15(4): 341-349.

van Noorden, G. E., Kerim, T., Goffard, N., Wiblin, R., Pellerone, F. I., Rolfe, B. G. \& Mathesius, U. (2007). Overlap of proteome changes in Medicago truncatula in response to auxin and Sinorhizobium meliloti. Plant Physiology 144(2): 1115-1131.

van Zeijl, A., den Camp, R. H. O., Deinum, E. E., Charnikhova, T., Franssen, H., den Camp, H. J. O., Bouwmeester, H., Kohlen, W., Bisseling, T. \& Geurts, R. (2015). Rhizobium lipo-chitooligosaccharide signaling triggers accumulation of cytokinins in Medicago truncatula roots. Molecular Plant 8(8): 1213-1226.

Vance, C. P. (2001). Symbiotic nitrogen fixation and phosphorus acquisition. Plant nutrition in a world of declining renewable resources. Plant Physiology 127(2): 390-397. 
Varma Penmetsa, R., Uribe, P., Anderson, J., Lichtenzveig, J., Gish, J. C., Nam, Y. W., Engstrom, E., Xu, K., Sckisel, G. \& Pereira, M. (2008). The Medicago truncatula ortholog of Arabidopsis EIN2, sickle, is a negative regulator of symbiotic and pathogenic microbial associations. The Plant Journal 55(4): 580-595.

Vernié, T., Kim, J., Frances, L., Ding, Y., Sun, J., Guan, D., Niebel, A., Gifford, M. L., de Carvalho-Niebel, F. \& Oldroyd, G. E. (2015). The NIN Transcription Factor Coordinates Diverse Nodulation Programs in Different Tissues of the Medicago truncatula Root. The Plant cell 27(12): 3410-3424.

Vessey, J. K., Pawlowski, K. \& Bergman, B. (2004). Root-based N-2-fixing symbioses: Legumes, actinorhizal plants, Parasponia sp and cycads. Plant and Soil 266(1-2): 205-230.

Wang, C., Zhu, H., Jin, L., Chen, T., Wang, L., Kang, H., Hong, Z. \& Zhang, Z. (2013). Splice variants of the SIP1 transcripts play a role in nodule organogenesis in Lotus japonicus. Plant Molecular Biology 82(1-2): 97111.

Wang, H., Moore, M. J., Soltis, P. S., Bell, C. D., Brockington, S. F., Alexandre, R., Davis, C. C., Latvis, M., Manchester, S. R. \& Soltis, D. E. (2009). Rosid radiation and the rapid rise of angiosperm-dominated forests. Proceedings of the National Academy of Sciences 106(10): 3853-3858.

Webster, G., Poulton, P., Cocking, E. \& Davey, M. (1995). The nodulation of micro-propagated plants of Parasponia andersonii by tropical legume rhizobia. Journal of Experimental Botany 46(9): 1131-1137.

Wen, X., Zhang, C., Ji, Y., Zhao, Q., He, W., An, F., Jiang, L. \& Guo, H. (2012). Activation of ethylene signaling is mediated by nuclear translocation of the cleaved EIN2 carboxyl terminus. Cell Research 22(11): $1613-$ 1616.

White, F., Taylor, B., Huffman, G., Gordon, M. \& Nester, E. (1985). Molecular and genetic analysis of the transferred DNA regions of the root-inducing plasmid of Agrobacterium rhizogenes. Journal of bacteriology 164(1): 33-44.

Wopereis, J., Pajuelo, E., Dazzo, F. B., Jiang, Q., Gresshoff, P. M., De Bruijn, F. J., Stougaard, J. \& Szczyglowski, K. (2000). Short root mutant of Lotus japonicus with a dramatically altered symbiotic phenotype. The Plant Journal 23(1): 97-114.

Xiao, T. T., Schilderink, S., Moling, S., Deinum, E. E., Kondorosi, E., Franssen, H., Kulikova, O., Niebel, A. \& Bisseling, T. (2014). Fate map of Medicago truncatula root nodules. Development 141(18): 3517-3528.

Yang, D.-L., Yang, Y. \& He, Z. (2013). Roles of plant hormones and their interplay in rice immunity. Molecular Plant 6(3): 675-685.

Yang, M.-Q., van Velzen, R., Bakker, F. T., Sattarian, A., Li, D.-Z. \& Yi, T.-S. (2013). Molecular phylogenetics and character evolution of Cannabaceae. Taxon 62(3): 473-485.

Yano, K., Yoshida, S., Müller, J., Singh, S., Banba, M., Vickers, K., Markmann, K., White, C., Schuller, B. \& Sato, S. (2008). CYCLOPS, a mediator of symbiotic intracellular accommodation. Proceedings of the National Academy of Sciences 105(51): 20540-20545.

Yesson, C., Russell, S., Parrish, T., Dalling, J. \& Garwood, N. (2004). Phylogenetic framework for Trema (Celtidaceae). Plant Systematics and Evolution 248(1-4): 85-109. 


\section{Chapter 7}

Yokota, K., Soyano, T., Kouchi, H. \& Hayashi, M. (2010). Function of GRAS proteins in root nodule symbiosis is retained in homologs of a non-legume, rice. Plant and Cell Physiology 51(9): 1436-1442.

Yoneyama, K., Xie, X., Kim, H. I., Kisugi, T., Nomura, T., Sekimoto, H., Yokota, T. \& Yoneyama, K. (2012). How do nitrogen and phosphorus deficiencies affect strigolactone production and exudation? Planta 235(6): 1197-1207.

Young, N. D., Debellé, F., Oldroyd, G. E., Geurts, R., Cannon, S. B., Udvardi, M. K., Benedito, V. A., Mayer, K. F., Gouzy, J. \& Schoof, H. (2011). The Medicago genome provides insight into the evolution of rhizobial symbioses. Nature 480(7378): 520-524.

Yu, Y.-B., Adams, D. O. \& Yang, S. F. (1979). 1-Aminocyclopropanecarboxylate synthase, a key enzyme in ethylene biosynthesis. Archives of biochemistry and biophysics 198(1): 280-286.

Zhang, X.-C., Millet, Y. A., Cheng, Z., Bush, J. \& Ausubel, F. M. (2015). Jasmonate signalling in Arabidopsis involves SGT1b-HSP70-HSP90 chaperone complexes. Nature Plants 1(5). 


\section{SUMMARY}

Bacteria of the genus Rhizobium play a very important role in agriculture by inducing nitrogenfixing nodules on the roots of legumes. Root nodule symbiosis enables nitrogen-fixing bacteria to convert atmospheric nitrogen into a form that is directly available for plant growth. This symbiosis can relieve the requirements for added nitrogenous fertilizer during the growth of leguminous crops. Establishment of the rhizobium-legume symbiosis depends on a molecular dialogue, in which rhizobial nodulation (Nod) factors act as symbiotic signals, playing a key role in the control of specificity of infection and nodule formation. Bacterial and legume genes involved in establishing and maintaining the symbiosis are studying over the decades. The expression of "nodulation" genes in the bacteria is activated by signals from plant roots and as a result the bacteria synthesise signals that induce a nodule meristem and enable the bacteria to enter and engage with the host plant.

Research on legume-rhizobium symbioses has emphasized fitness benefits to plants but in our research, we take a different vantage point, focusing on the Parasponia-rhizobium symbiosis. Parasponia is the only non-legume plant capable of establishing mutualistic relation with rhizobia. This study will provide background knowledge for use in applied objectives as well as yielding a wealth of fundamental knowledge with wide implications from rhizobium symbiosis evolution. Underpinning the work is a continuing investigation of the genes specifically induced during the symbiosis. The communications that occur between the plant and the rhizobia during nodule formation and maintenance constitutes a novel opportunity to study signal transduction in a plant system.

This thesis describes my research on genetic constrains that determine rhizobium-root nodule formation. To identify these constraints we used Parasponi anadersnii as only non-legume capable to establish nitrogen fixing rhizobium symbiosis. Our main attempt in this thesis was to find the genetic constraints using Parasponia as a key and reconstruct an auto active symbiotic signaling cascade in the non- legume plants.

To facilitate the identification of symbiotic genes in Parasponia, first we developed methods to generate transgenic plants. In line with this, a simple and efficient hairy root transformation method was established in Chapter 2. We consider this is an improved protocol of Agrobacterium rhizogenes-mediated transformation. In about 1 months of in vitro culture, we 


\section{Summary}

could recover a high number of transgenic Parasponia plants that were resulted from independent transformation.

To determine the genetic elements that underlie the rhizobium symbiosis, we aimed to compare Parasponia with closest non nodulating specious, Trema tomentosa. To do so, we developed an efficient genetic transformation method for Trema mediated by Agrobacterium tumefaciens in Chapter 3. With this protocol we could able to produce stable transgenic line in 6 month. The entire procedure for generating transgenic plants achieved a transformation frequency of $15 \%$ which was sufficiently efficient to conduct experiments at larger scale.

Negative effect of nitrate on root nodulation of legumes has been commonly reported. When legume plants are supplied with nitrate, nodule formation, nodule development and $\mathrm{N}_{2}$ fixation activity all inhibited. With support of this finding in legumes, we implemented in a physiological study on symbiotic response of Parasponia to nitrate. This research opened a novel view on the Parasponia-rhizobium symbiosis by discovering a different mechanism that control root nodule formation in Parasponia in compare with legumes. In Chapter 4 we showed that, although legume root nodule formation is inhibited in moderate to high concentration of the nitrate, Parasponia forms nodule even in $50 \mathrm{mM}$ nitrate availability. However intracellular infection is markedly reduced. This suggests that Parasponai-rhizbium symbiosis is not evolved to regulate the nodule number in presence of the nitrate. In fact the lack of making balance between the need to fixed nitrogen and energy cost to supply rhizobium requirements cause nodule formation in nitrate availability. These results indicate new evidence that Parasponia-rhizobium symbiosis evolved recently.

According to the fact that Parasponia and legumes are remotely related, it was hypothesized that, Parasponia-rhizobium symbiosis evolved independently. Therefore we put forward our attempt to determine the genes required for nodule formation in Parasponia. by extending our research on symbiotic genes which are available in non nodulating plants with different function, namely NSP1 and NSP2. It is known for a decade that NSP1 and NSP2 genes are transcription factors essential for rhizobial nod factor induction in legumes. In Chapter $\mathbf{5}$ we described the role of NSP genes during nodulation and mycorrhization in Parasponia. Performing NSP1 and NSP2 RNAi knocked down Parasponia plants showed that these genes positively regulate Root Nodule (RN) and Amrbuscule Mycorrizal (AM) formation in Parasponia. Mutation in either 
NSP1 or NSP2 markedly reduced nodule formation. It has been previously shown in legumes that NSP proteins have a function in the interaction of plants with AM symbiosis. In the same fashion, in our research knock down mutation in NSPs displayed reduced mycorrhizal colonization level. This shows that NSP1 and NSP2 are involved in both nodulation and mycorrhization. This result highlight the idea that RN and AM symbiosis are conserved in part of the pathway and probably bifurcates into two branches by NSP transcription factor allowing specific activation of nodulation or mycorrhization.

Aiming to know the role of hormones in symbiotic behavior, we focused on ethylene as a negative regulator of nodule formation in legumes in Chapter 6. We found the negative effect of ethylene on root nodulation of Parasponia. For the first time we reported a hyper nodulation (20 fold nodule number in compare with control plants) phenotype in Parasponia by performing knocked down mutant of EIN2 gene, a key regulator of ethylene signaling pathway. Further, in a focused approach we investigated the functional behaviour of EIN2 of Trema, a close relative of Parasponia that does not fix nitrogen. EIN2 knocked down mutant of Trema formed nodule like structures in the roots. This result indicated that at least part of the nodule formation capacity in Parasponia has been recruited by ethylene signaling pathway during evolution.

Finally, the results obtained in this study provide new insight into the fact that rhizobium symbiosis are under tight genetic constraints that guide endosymbiosis in remotely evolved host plants, legumes and Parasponia. Considering this finding along with comparison of Parasponia with closest non nodulating sister species, Trema, will result in determination of genetic constraints which underlay rhizobium symbiosis. Finding these constraints will help to uncover the core elements and transfer this important trait to major important non legume plants.

In Chapter 7 the main achievements of this study are discussed and directions for future experiments are highlighted. 


\section{Acknowledgements}

I wish to express my gratitude to those who understand and support has considerably contributed to the completion of this thesis. This goes with the sincere hope that I have been showing my appreciation through actions all this time. It is also a challenge because words are not always enough to express my appreciation. The $\mathrm{PhD}$ study is a journey with lots of twists and turns that is impossible to finish without the input of many people who play an important and unique role along the road.

I would first like to thank my supervisor Prof. Dr. Ton Bisseling, who gave me the opportunity to work with his research team. He has been a fantastic mentor, role model, and friend during my doctoral research. I feel extremely fortunate to have a chance to work with him and to benefit from his diverse expertise for the completion of this thesis.

I would like to express my thanks to Dr Rene Geurts, my co-promotor, for his input and drive. Rene, your door was always open for me and I never felt alone in taking important decisions. Thank you for making my $\mathrm{PhD}$ a continuous learning process and for allowing me to explore possibilities other than my PhD work.

Particular thanks must be extended to Marie-Jose and Maria, I deeply appreciate your patience, understanding and helping me in all difficult situations that I had. You always gave me motivation even when the direction was not clear for me. I never forget your open hugs and smiling face for all my requests.

I would like to thank Elisa Polone and Rik op den camp for their help with starting my PhD. Elisa you were very kind and patient. We had lots of discussion around different topics. Now you are mom and lucky your beautiful baby for having such a nice mom. 


\section{Summary}

Rik, I learned a lot from you and always admire your courage and intelligence. You thought me equipment in many circumstances, and I wish you to know that without this, I would still need a few more years to make things happen.

I wish to thank Staff of the chair Molecular Biology; Elena, Henk, Carolien, Marijke, Jan, Olga, Ludmilla, Erik, Jan and Joan for sharing space and thoughts as well as for making the lab and office a pleasant place to be and work.

I would like to thanks Wim Roelofsen for his useful contributions in this thesis. It was always nice working with him in biochemistry lab.

Moreover, I would like to thank my officemates and colleagues during my PhD. Stefan, Gerben, Arend, Wei, Sergey, Alessandra, Sjef, Aleksandr, Eva, Evgenia, Adam, Rik, Vid, Juliane, TingTing, Trupti, Arjan, sergul, Qingqin, Robin, Wouter, Fengjiao, Huchen, Guiling, Defeng and Tian. I was lucky that you were my officemate, we had many fun with together that made $\mathrm{PhD}$ joyful to me.

I am deeply indebted to my student, Wouter van schooten, I wish you success for your study career and your personal life

I would like to express my special appreciations to all the Iranian friends in Wageningen that have made our stay in Wageningen a very pleasant time to me and my family. The time that I spent with you during parties, trips and other occasions are unforgettable to me. Since, I am afraid to miss some names I want just to tell that I love you all.

I wish to thank my parents, my brothers and my sisters for their support and encouragement throughout my life and during my $\mathrm{PhD}$ study.

I kept my special thanks for my best friend, my husband, Sasan. We fell in love in a scientific environment and grown this love in special atmosphere full of joy 
and shared experiences. Our main part of life in Wageningen spent in the lab and in every break, we always discussed about the experiments and results.

Sasan you are a great scientist, sometime I was jealous that you love science more than me! But on the other hand was proud of having so intelligence husband. I never forget that being Phd and having such a great experience was never possible without your support and Encouragement, many love for you forever. 


\begin{abstract}
About the author
Maryam Seifi Kalhor was born on $20^{\text {nd }}$ January 1981 in Qom, Iran. After completing high school in his home town he started his higher education in 1999 and finished his bachelor in Plant breeding in 2004 at University of Ilam. She started her MSc-program in Biotechnology at Tabriz University in 2005 and received her MSc certificate with distinguished degree in 2007. She accomplished several research studies funded by different institutes between 2008 and 2010. One of her researches with the title of "improving tolerance to boron toxicity by using of salicylic acid in peppermint" was selected as best national research plans in 2010. After one year she started her PhD in 2011 in Wageningen University, the Netherlands. During her PhD she worked on the Parasponia plants and its symbiotic interaction with rhizobium to find genetic constraints underlying this symbiosis. The result of this study is presented in this thesis.
\end{abstract}


Issued to: Maryam Seifi Kalhor

Date:

Group:

Laboratory of Molecular Biology

University: Wageningen University \& Research

\section{1) Start-up phase}

First presentation of your project

Title: Genetic constraints that determine Rhizobium-root nodule formation in Parasponia

Writing or rewriting a project proposal

Title: Genetic contrains in evolution of a Rhizobium symbiosis

- Writing a review or book chapter

MSc courses

Bioinformatics

Laboratory use of isotopes

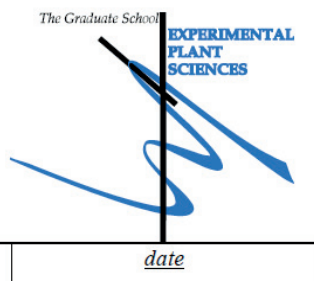

Jan 10,2011

Aug 2012

2012

13.5 credits $^{*}$

date

2) Scientific Exposure

- EPS $\mathrm{PhD}$ student days

$\mathrm{EPS} \mathrm{PhD}$ student day, Wageningen University

EPS PhD student day, University of Amsterdam

- EPS theme symposia

Annual EPS Theme 1 symposium 'Developmental Biology of Plants', Leiden University

Annual EPS Theme 1 symposium 'Developmental Biology of Plants', Wageningen University \&

Annual EPS Theme 1 symposium 'Developmental Biology of Plants', Leiden University

Annual EPS Theme 4 symposium 'Genome Biology', Wageningen University \& Research

Annual EPS Theme 1 symposium 'Developmental Biology of Plants', Wageningen University \&

- Lunteren days and other National Platforms

Annual Meeting 'Experimental Plant Sciences', Lunteren, NL

Annual Meeting 'Experimental Plant Sciences', Lunteren, NL

Annual Meeting 'Experimental Plant Sciences', Lunteren, NL

- Seminars (series), workshops and symposia

Symposium 'Plant Breeding in the Genomics Era'

Plant Sciences Seminar on Bioinformatics

EPS Flying Seminar Prof. Graham Farquhar

Seminar David M. Weller (Root Disease and Biological Control Research Unit)

Green Life Sciences seminar Prof. Holger Puchta

WEES Seminar Eric Schranz (Whole genome duplications as drivers of evolutionary)

Wageningen $\mathrm{PhD}$ Symposium

Open Symposium: Plant Metabolomics

Invited seminar Jos Raaijmakers (exploring and exploiting the plant microbiome)

- International symposia and congresses

MiCom 2012: International Student Conference on Microbial Communication, Jena, Germany

EPSO 2013 : International Conference, Porto Heli, Greece

Plant transformation technolgy III conference, Vienna, Austria

- Presentations

MiCom 2012: International Student Conference on Microbial Communication (Poster)

EPSO 2013 : International Student Conference (Poster)

Plant transformation technolgy III conference (Poster)

LAB interview

Excursions

Suhtotal Sciontific Frnocure

3) In-Depth Studies

- EPS courses or other $\mathrm{PhD}$ courses

Bioinformatics - a User's Approach

Generalized linear model

- Journal club

Member of literature discussion group at laboratory of Molecular Biology

- Individual research training

\begin{tabular}{|c|c|}
\hline & date \\
& Mar 04-08,2013 \\
Jun 14-15, 2012 \\
gy & $2010-2014$ \\
\hline
\end{tabular}

Subtotal In-Depth Studies

4) Personal development

- Skill training courses

How to Write a World Class Paper

Techniques for Writing and Presenting a Scientific Paper

Scientific publishing

WGS course 'Interpersonal Communication for $\mathrm{PhD}$ Students

- Organisation of $\mathrm{PhD}$ students day, course or conference

Membership of Board, Committee or PhD council
5.1 credits $^{*}$

May 20, 2011

Nov 29, 2012

Jan 20, 2011

Jan 19,2012

Jan 17, 2013

Dec 13, 2013

Jan 24, 2014

Apr 04-05, 2011

Apr 22-23, 2013

Apr 14-15, 2014

Nov 25, 2011

Mar 12, 2013

Mar 13, 2013

Sep 25, 2013

Sep 26, 2013

Nov 20, 2013

Dec 10, 2013

Dec 12, 2013

Jan 07, 2014

Nov 05-08, 2012

Sep 01-04, 2013

Feb 12-15, 2014

Nov 05-08, 2012

Sep 01-04, 2013

Feb 12-15, 2014

tho

\section{date}

Oct 26,2010

Jul 03-06, 2102

Jun 19, 2012

Apr 10-11, 2014

$2.2 \operatorname{credits}^{3}$

\section{TOTAL NUMBER OF CREDIT POINTS*}

Herewith the Graduate School declares that the $\mathrm{PhD}$ candidate has complied with the educational requirements set by the Educational Committee of EPS which comprises of a minimum total of 30 ECTS credits

* A credit represents a normative study load of 28 hours of study. 
Lay-out: Marym Seifi kalhor

Cover: Designed by Sasan Ali Niaei Fard and Maryam Seifi kalhor

Parasponia andersonii (WU1) transgenic roots (front) and Parasponia andersonii (WU1) nodule micro-section photo (back). 